\title{
Numerical and Theoretical Investigation of The Dynamic Response of Corrugated Core Sandwich Columns
}

\author{
A Dissertation \\ Presented to \\ the Faculty of the School of Engineering and Applied Science \\ University of Virginia \\ In Partial Fulfillment \\ of the requirements for the Degree \\ Doctor of Philosophy in Mechanical and Aerospace Engineering \\ by
}

Jae-Yong Lim

December 2012 
(C) Copyright by

Jae-Yong Lim

All Rights Reserved

December 2012 


\section{APPROVAL SHEET}

The dissertation is submitted in partial fulfillment of the requirements for the degree of

Doctor of Philosophy in Mechanical and Aerospace Engineering

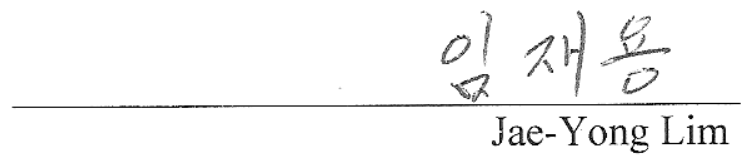

This dissertation has been read and approved by the examining Committee:
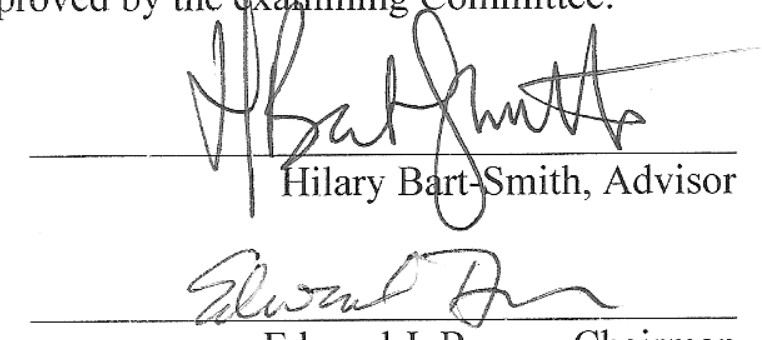

Edward J. Berger, Chairman
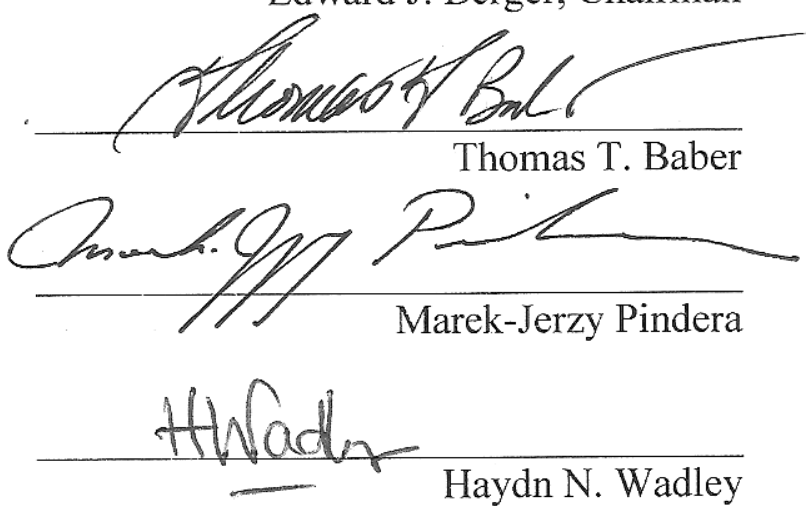

Accepted for the School of Engineering and Applied Science:

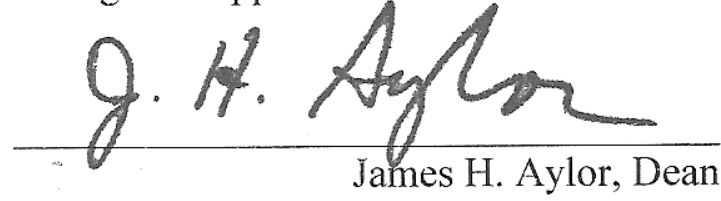

School of Engineering and Applied Science

December, 2012 


\begin{abstract}
Complex dynamic effects influence the structural response of metallic core sandwich structures. Before these structures can be used in the field, it is necessary to understand the mechanical response and failure mechanisms of the sandwich structures under possible dynamic loading conditions which they may be subjected to. In this dissertation, a corrugated core sandwich structure under dynamic in-plane compression is the focus.
\end{abstract}

The main objective of this dissertation is to investigate the structural response of the Al6061-T6 and SS304 corrugated core sandwich columns under dynamic loading, through theoretical and numerical analysis. There are two in-plane loading directions, perpendicular-to-corrugations and parallel-to-corrugations, to accommodate the anisotropic response. The investigation considers compression velocities ranging from quasi-static up to $100 \mathrm{~m} / \mathrm{s}$, divided into low (up to the order of a few $\mathrm{m} / \mathrm{s}$ ) and high (the order of tens of $\mathrm{m} / \mathrm{s}$ ) velocity responses.

For low velocity response, analytical models are proposed to predict individual failure modes. Each model is dedicated to the prediction of each of the individual failure modes: global buckling failure, face wrinkling failure for sandwich columns compressed perpendicular-to-corrugations, the local plate buckling for sandwich columns compressed parallel-to-corrugations. All of those models, based on the theory of stress wave propagation, calculate the out-of-plane displacements until failure criteria are satisfied. The validity of the proposed models is confirmed by comparison with FE simulations. The models successfully describe complex phenomena such as dynamic 
strengthening by material strain-rate effects, inertia effects as well as imperfection sensitivity.

Using those analytical models, the dynamic effects on the failure maps are investigated. Subsequently it is successfully proven that increased rate-of-loading leads to the inertial stabilization of global buckling motion and the change of failure modes from global to local buckling.

The efficiency of the developed analytical models is highlighted in a dynamic optimization procedure. Due to the complex dynamic phenomena, the individual failure responses under low compression velocities of $\mathrm{V}=0.1$ and $1 \mathrm{~m} / \mathrm{s}$ are approximated as a function of sandwich design parameters, which is referred to as response surface methodology. A number of numerical experiments for the response approximation are calculated using the developed analytical models, and the optimization problems are solved via a sequential quadratic programming algorithm. As a result, it is concluded that sandwich columns are superior to monolithic columns, and that beneficial sandwich concepts are more remarkable at the lower velocities due to the inertial stabilization of global buckling motion. Moreover, it is suggested that the minimum weight design of corrugated core sandwich columns with maximum impulse capacity must consider reinforcing local buckling strength under dynamic loading.

Lastly, the high velocity sandwich column response is generalized in terms of sandwich geometric dimensions and loading intensity. In this high velocity response region where considered compression velocity is of the order of tens of $\mathrm{m} / \mathrm{s}$, the response time scale is of the order of less than one round trip of a plastic wave. Thus, the high 
velocity column response can be accounted for by applying the theory of rateindependent elastic-plastic wave propagation with an analogy between monolithic solid columns and sandwich columns. Simplified theoretical models for each in-plane loading orientation are suggested and validated by FEM. 


\section{TABLE OF CONTENTS}

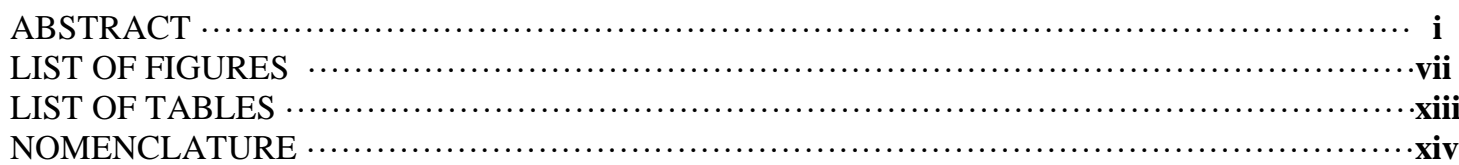

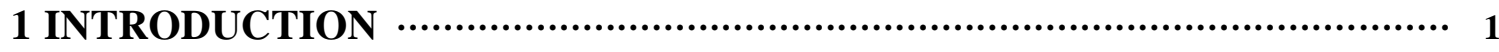

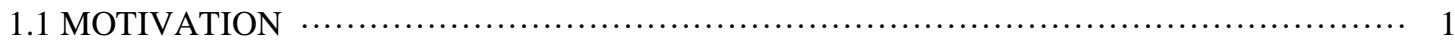

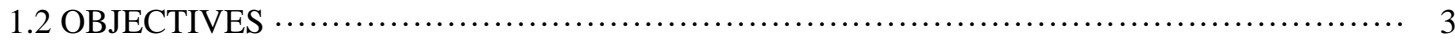

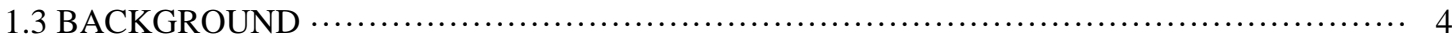

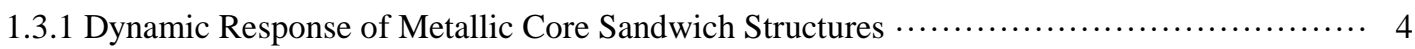

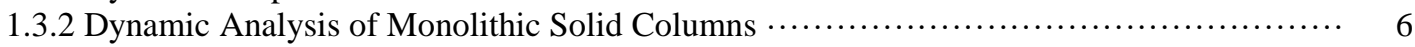

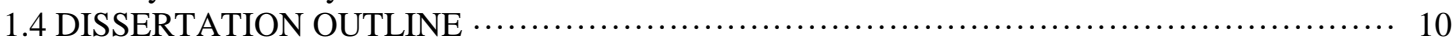

\section{AN ANALYTICAL MODEL FOR THE PREDICTION OF GLOBAL

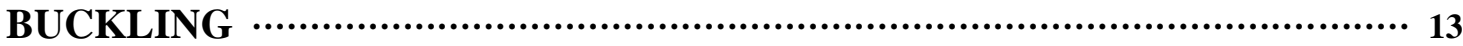

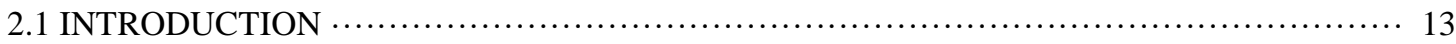

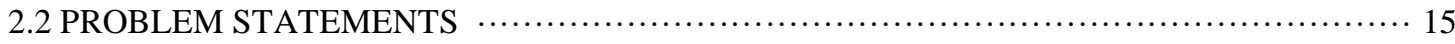

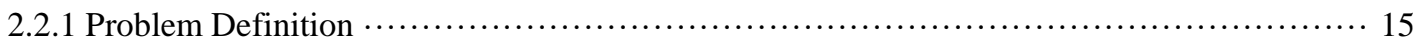

2.2.2 Formulation for Monolithic Solid Columns $\ldots \ldots \ldots \ldots \ldots \ldots \ldots \ldots \ldots \ldots \ldots \ldots \ldots \ldots \ldots \ldots \ldots$

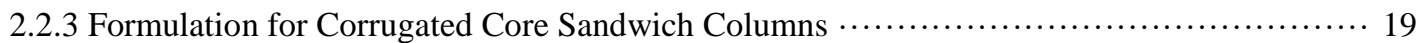

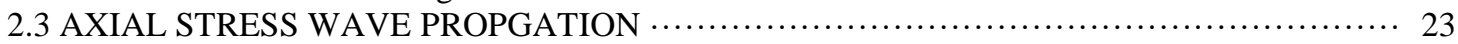

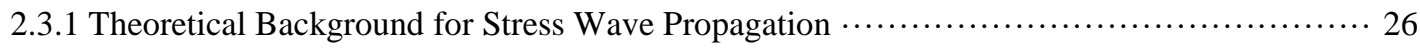

2.3.2 Analogy between Monolithic Solid Columns and Sandwich Columns .................... 29

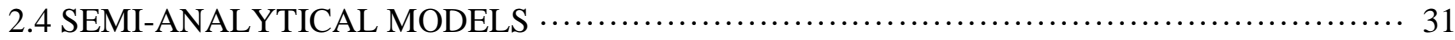

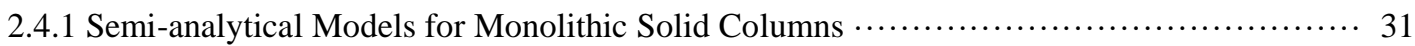

2.4.2 Semi-analytical Models for Corrugated Core Sandwich Columns ...................... 35

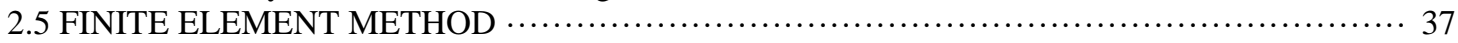

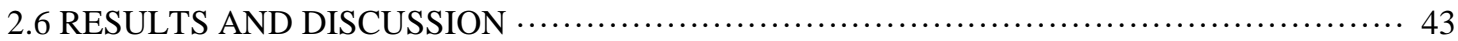

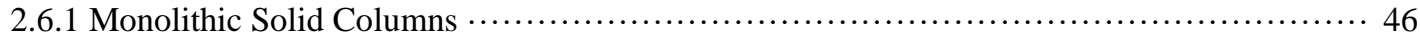

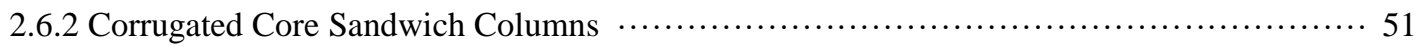

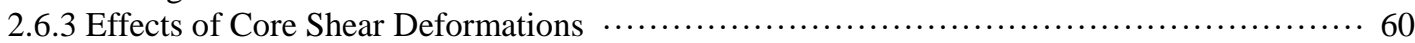

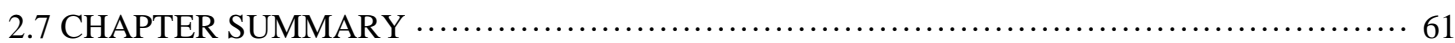

3 AN ANALYTICAL MODEL FOR THE PREDICTION OF FACE WRINKLING

...........................................................................................................64 64

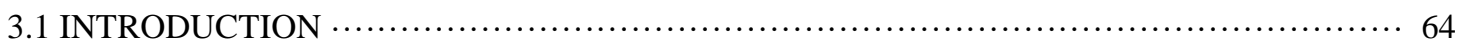

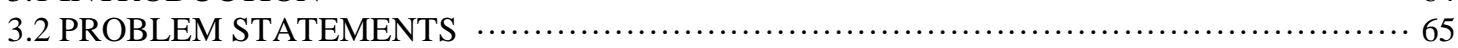

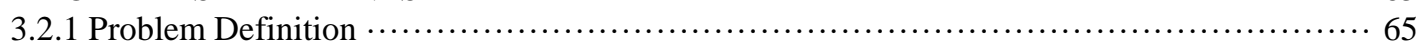

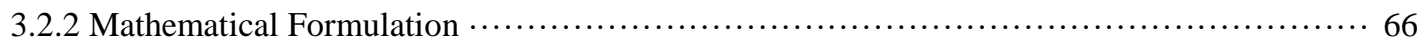

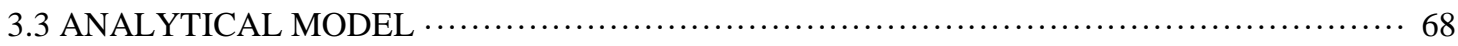

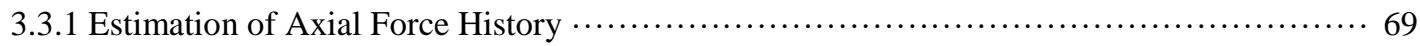

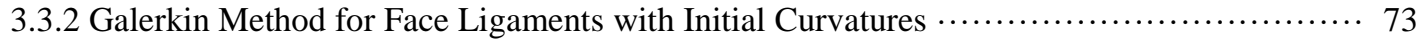

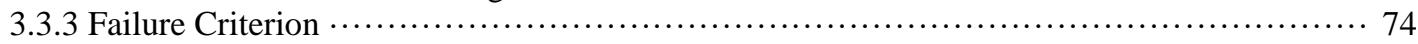

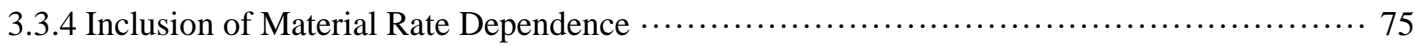

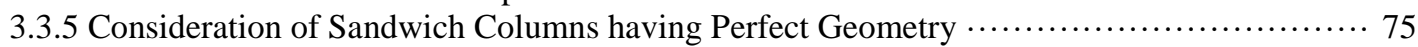

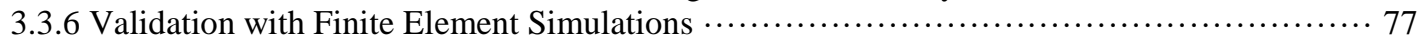




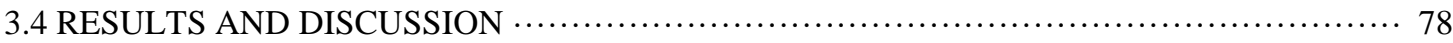

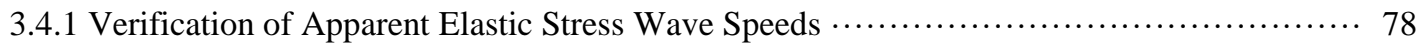

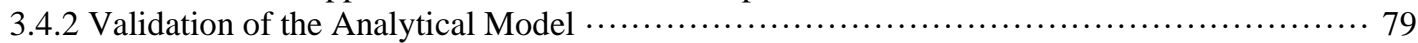

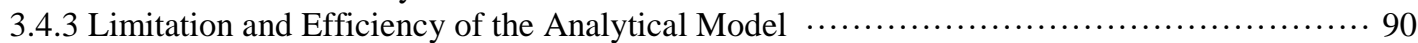

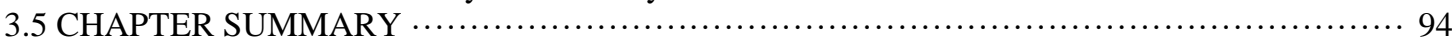

4 AN ANALYTICAL APPROACH FOR THE PREDICTION OF LOCAL PLATE

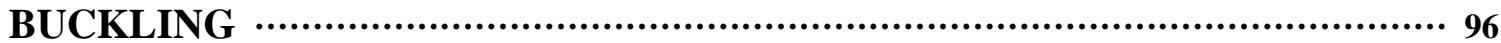

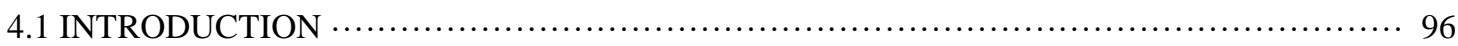

4.2 FE OBSERVATIONS OF LOCAL PLATE BUCKLING OF SANDWICH COLUMNS …… 97

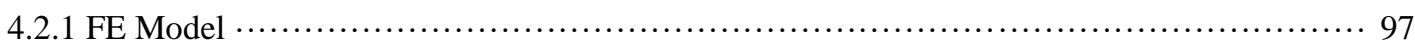

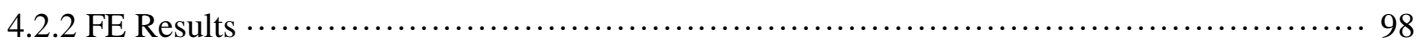

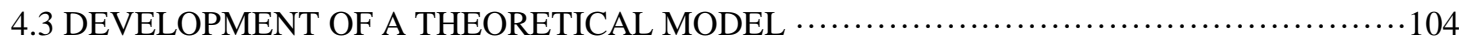

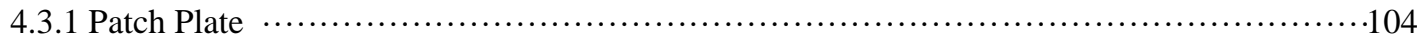

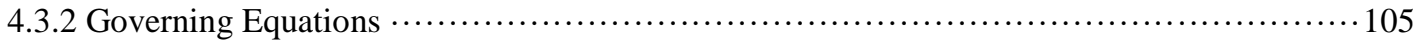

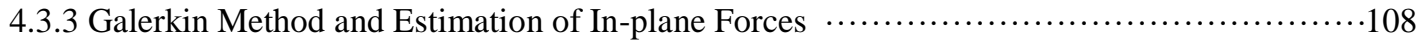

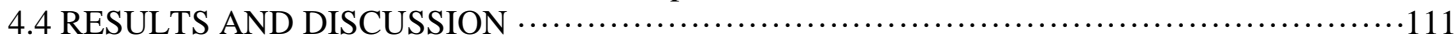

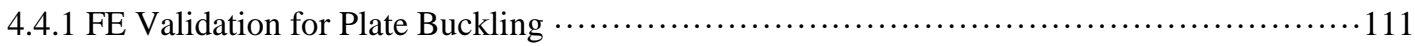

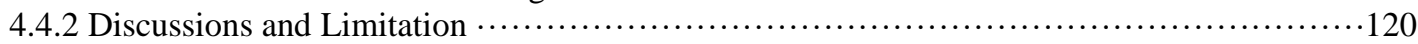

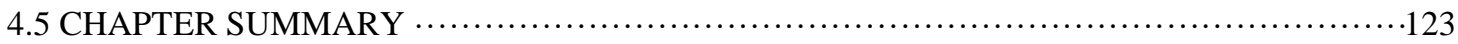

5 DYNAMIC FAILURE MECHANISM MAPS………………………………...125

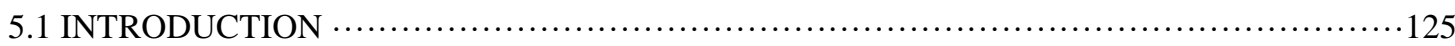

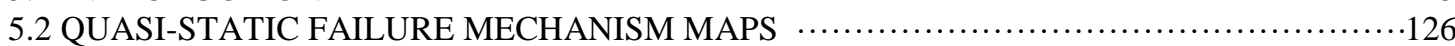

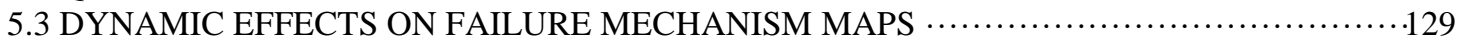

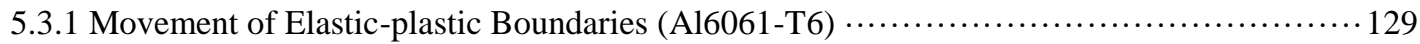

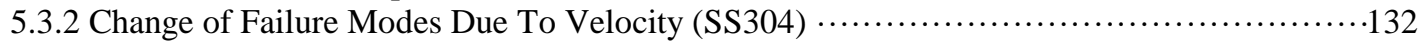

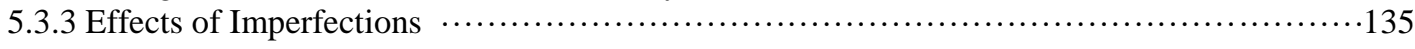

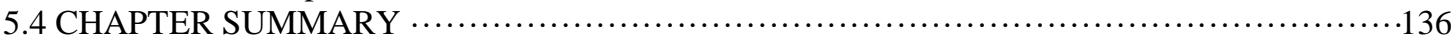

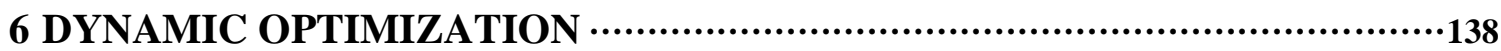

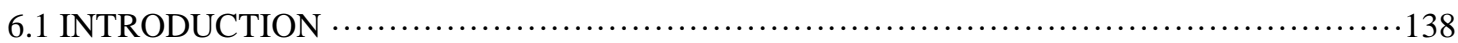

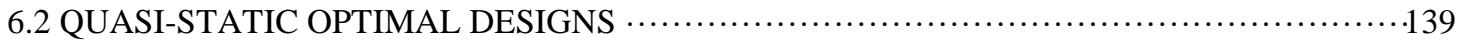

6.3 DYNAMIC RESPONSE OF QUASI-STATIC OPTIMAL SOLUTIONS AND THEIR MASS-

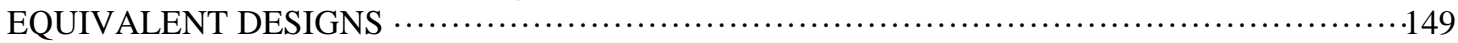

6.4 DYNAMIC OPTIMIZATION FOR AL6061-T6 SANDWICH COLUMNS ….................... 159

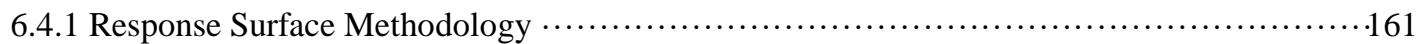

6.4.2 Optimization of Al6061-T6 Sandwich Columns Under Dynamic In-plane Loading …....... 167

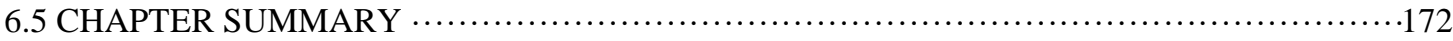

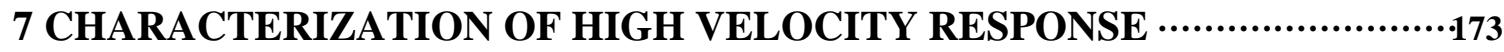

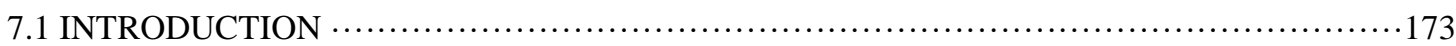

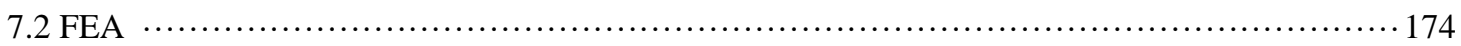

7.3 HIGH VELOCITY RESPONSE OF SANDWICH COLUMNS (PERPENDICULAR-TO-

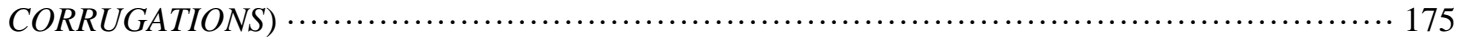

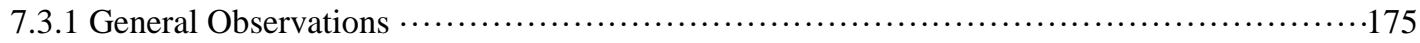

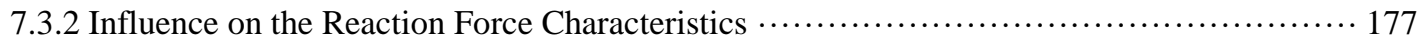

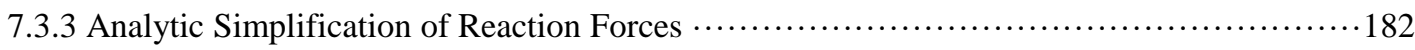

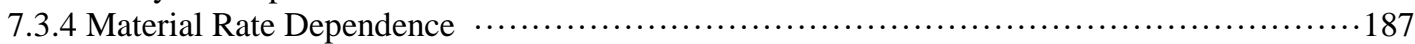


7.4 HIGH VELOCITY RESPONSE OF SANDWICH COLUMNS (PARALLEL-TO-

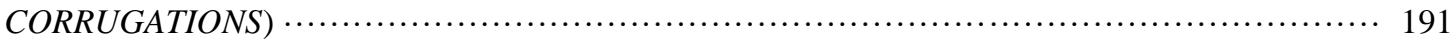

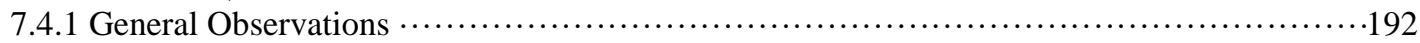

7.4.2 Influence on the Reaction Force Characteristics …................................. 194

7.4.3 Analytic Simplification of Reaction Forces …........................................ 197

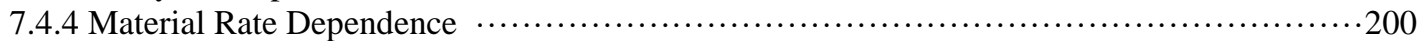

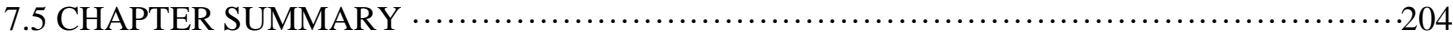

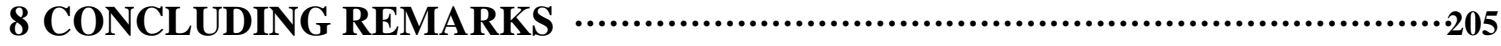

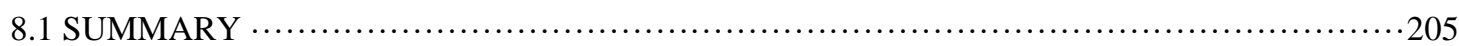

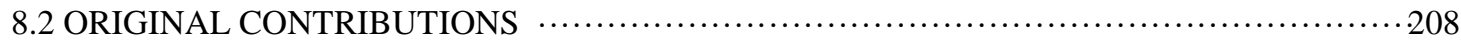

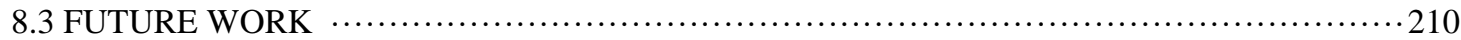

REFERENCES

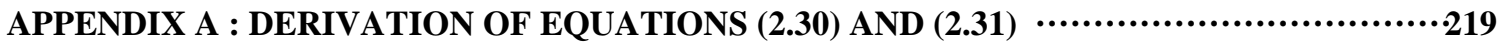

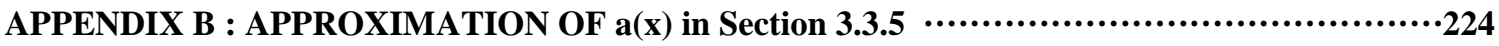

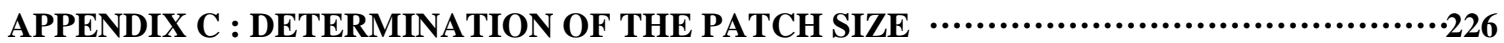

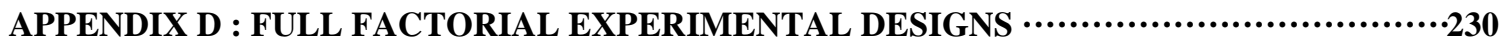

APPENDIX E : EQUIVALENT CORE STIFFNESS OF THE CORE STRUT CLOSEST TO THE

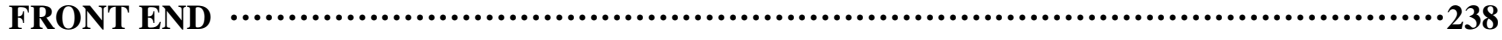

APPENDIX F : WAVE PROPAGATION IN MATERIAL WITH BILINEAR PLASTIC PARTS 240 


\section{List of Figures}

1.1 In-plane loading orientations: (a) Perpendicular-to-corrugations; (b) Parallel-to-corrugations . 4

2.1 Three types of dynamic problems: (a) monolithic solid column; (b) corrugated core sandwich column compressed perpendicular-to-corrugations; (c) corrugated core sandwich column

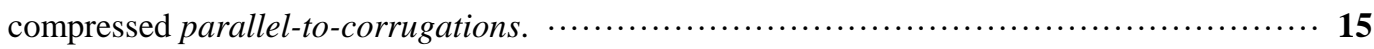

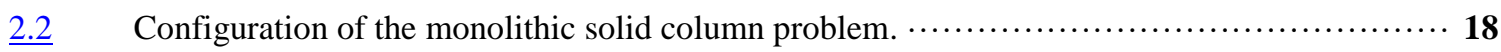

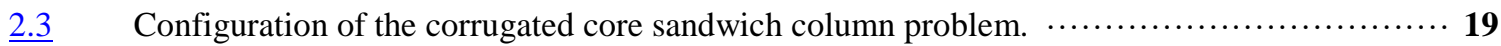

2.4 Stress distribution diagram (monolithic solid columns), Lagrange $x-t$ diagram and the corresponding material state for $(a)(b)(c)$ an elastic stress wave propagation, and (d)(e)(f) an

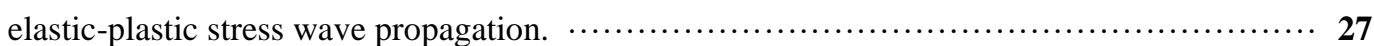

2.5 Stress distribution diagram (sandwich columns), Lagrange x-t diagram and the corresponding material state for (a)(b)(c) an elastic stress wave propagation, and (d)(e)(f) an elastic-plastic stress

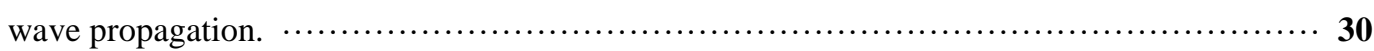

2.6 Flow chart of the semi-analytical model for monolithic solid columns. $\ldots \ldots \ldots \ldots \ldots \ldots \ldots \ldots \ldots \ldots \ldots \ldots \ldots \ldots$

2.7 FE model of Al6061-T6 sandwich columns compressed perpendicular-to-corrugations......... 38

2.8 FE model of Al6061-T6 sandwich columns compressed parallel-to-corrugations............... 38

2.9 FE unitcell model for A16061-T6 sandwich columns with face sheets meshed by (a) continuum

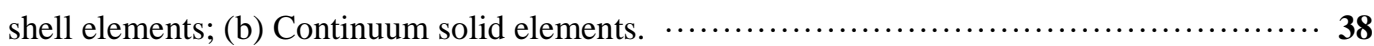

2.10 FE model of SS304 sandwich columns compressed; (a) perpendicular-to-corrugations, (b)

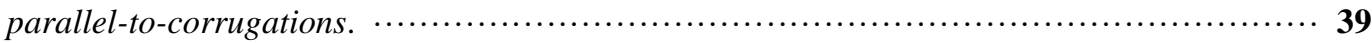

2.11 Quasi-static stress-strain material behavior compared with the constitutive models of (a) AL6061-

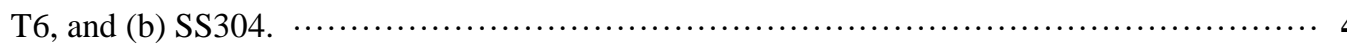

2.12 A constant velocity imposed on the front end of a sandwich column by a rigid plate. …..... 42

2.13 (a) Schematic curves for the front and back end reaction forces during elastic wave propagation; (b) axial stress distribution within a column at times of $\mathrm{t}_{1 \mathrm{e}}, \mathrm{t}_{2 \mathrm{e}}, \mathrm{t}_{3 \mathrm{e}}$; (c) idealized and FE-observed

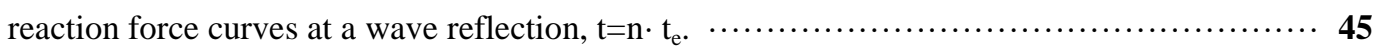

2.14 Dynamic response of an Al6061-T6 monolithic solid column of A=13.56mm, L=1407mm with $\xi=0.1905,0.9525,1.905$ under applied compressive velocities of 0.5 and $5 \mathrm{~m} / \mathrm{s}$ obtained from FEM and the semi-analytical model.

2.15 Dynamic response of a SS304 monolithic solid column of A=9.62mm, L=707mm with $\xi=0.884$ under compression velocities of 1 and $5 \mathrm{~m} / \mathrm{s}$ obtained from FEM and the semi-analytic model. $\mathbf{5 0}$

2.16 Dynamic response of an Al6061-T6 corrugated core sandwich columns (perpendicular-tocorrugations) under $\mathrm{V}=0.5$ and $5 \mathrm{~m} / \mathrm{s}$ obtained from FEM and the semi-analytical model....... 54 
2.17 Dynamic response of a SS304 corrugated core sandwich column (compressed perpendicular-tocorrugations) under applied compression velocities of $\mathrm{V}=1$ and $5 \mathrm{~m} / \mathrm{s}$ obtained from FEM and the semi-analytical model. 55

2.18 Dynamic response of an Al6061-T6 corrugated core sandwich column (parallel-to-corrugations) under compression velocities of 0.5 and $5 \mathrm{~m} / \mathrm{s}$.

2.19 Dynamic response of a SS304 corrugated core sandwich column under compression velocities of $\mathrm{V}=1$ and $5 \mathrm{~m} / \mathrm{s}$ obtained from FEM and the semi-analytical model.

2.20 The effect of core shear deformation for SS304 corrugated core sandwich columns under $\mathrm{V}=5 \mathrm{~m} / \mathrm{s}$ of (a) $\mathrm{h}=3.75 \mathrm{~mm}, \mathrm{t}=1.5 \mathrm{~mm}, \mathrm{l}=25.0 \mathrm{~mm}, \mathrm{w}=45^{\circ}, \bar{\rho}=0.12, \mathrm{~L}=707 \mathrm{~mm}$; and (b) $\mathrm{h}=3.75 \mathrm{~mm}$,

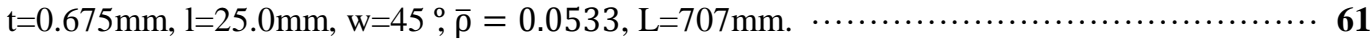

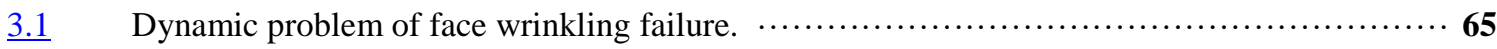

$\underline{3.2}$ Euler beam-column modeling of a face ligament for the dynamic face wrinkling. . . . . . . . . . $6 \mathbf{6 6}$

$\underline{3.3}$ Estimation of axial force history of face ligaments during elastic stress wave propagation. $\cdots \quad \mathbf{7 0}$

3.4 Estimation of axial force history of face ligaments during elastic-plastic stress wave propagation.

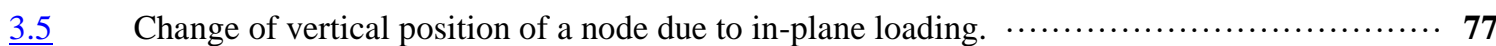

3.6 Apparent elastic stress wave speed $c_{e l}^{(\text {Perp })}$ of (a) A16061-T6 sandwich columns (L=1877mm, $\bar{\rho}=25 \%)$ and (b) SS304 sandwich columns ( $\mathrm{L}=2828 \mathrm{~mm}, \bar{\rho}=12 \%)$ of varied face sheet thickness, $\mathrm{h}$

3.7 Comparison between the FE and analytical calculations of the reaction force curves of the SS304 representative sandwich columns of (a) the EFW geometric dimensions under $\mathrm{V}=0.1 \mathrm{~m} / \mathrm{s}$, (b) the PFW geometric dimensions under V=1.0m/s; Deformation shapes of (c) the EFW obtained from the FE simulation at $\mathrm{t}=1400,1700 \mu \mathrm{s}$, and of (d) the PFW obtained from the FE simulation at $2800,3600 \mu \mathrm{s}$

3.8 Comparison between FE and analytical calculations for the reaction force curves of the Al6061-T6 representative sandwich columns of (a) the EFW geometric dimensions under $\mathrm{V}=0.5 \mathrm{~m} / \mathrm{s}$, (b) the PFW geometric dimensions under V=5.0m/s; Deformation shapes of (c) the EFW obtained from the FE simulation at $\mathrm{t}=600,800 \mu \mathrm{s}$, (d) the PFW obtained from the FE simulation at 240, $280 \mu \mathrm{s}$.

3.9 Plots of normalized peak reaction force, $P_{\text {peak }}^{(D y n)} / P_{c r}^{(Q S)}$ vs. V calculated by the FEM and analytical model

3.10 Transverse motion at the midpoint of a failed face ligament of the SS304 representative sandwich column of the EFW geometric dimensions under $\mathrm{V}=0.1 \mathrm{~m} / \mathrm{s}$ 
3.11 The effect of column length L on the dynamic response of SS304 corrugated core sandwich columns of the EFW geometric parameters having the same static strength, $P_{c r}^{(Q S)}$. 86

3.12 Influence of geometric imperfections. Reaction force versus time history curves calculated from FEM of (a) SS304 representative sandwich column of the EFW geometric dimensions under $\mathrm{V}=0.1 \mathrm{~m} / \mathrm{s}$, (b) Al6061-T6 representative sandwich column of the EFW geometric dimension under $\mathrm{V}=0.1 \mathrm{~m} / \mathrm{s}$. And the analytical model are compared against the FEA on the imperfection sensitive dynamic peak load and nominal strain at load drop of (c) the SS304 column and (d) the Al6061-T6 column.

88

3.13 (a) Dynamic simulation of the representative Al6061-T6 sandwich column of the EFW geometric dimensions (perfect geometry); (b) The first eigenmode; (c) The $7^{\text {th }}$ Eigenmode

3.14 Reaction force-time history curves at both front and back of an Al6061-T6 representative corrugated core sandwich column of the EFW geometric dimensions under (a) V=0.1m/s, (b)

$\mathrm{V}=0.5 \mathrm{~m} / \mathrm{s}$, (c) $\mathrm{V}=1.0 \mathrm{~m} / \mathrm{s}$; (d) The reaction force versus normalized time histories for the three compression rates.

3.15 Energy balance plot of the A16061-T6 representative sandwich column of the EFW geometric dimensions under $\mathrm{V}=0.1 \mathrm{~m} / \mathrm{s}$.

3.16 Contour of equivalent plastic strain, p, of the Al6061-T6 representative sandwich column of the

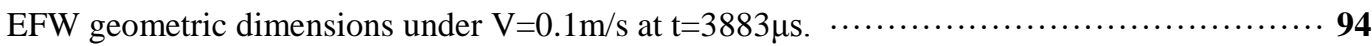

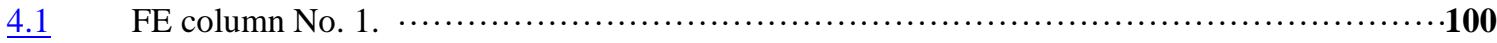

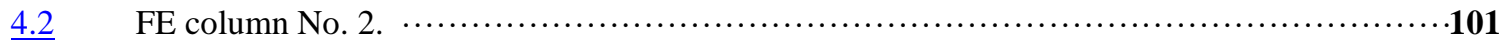

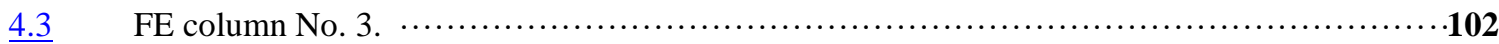

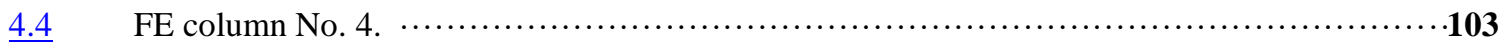

4.5 (a) Patch plates on a core plate of a corrugated core sandwich column; (b) Patch plates on a face plate of a corrugated core sandwich column; (c) Boundary conditions of the parch plates. $\cdots \cdot \mathbf{1 0 5}$

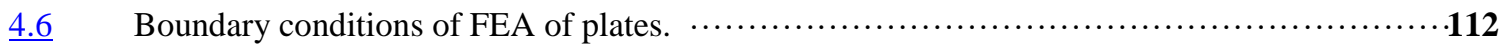

4.7 The assigned eigenmode shape superposed for initial curvature imperfections. $\cdots \ldots \ldots \ldots \ldots \ldots 113$

4.8 FE validation of the analytical model for the Al6061-T6 elastic plate (h:0.457mm, W: 22.0mm,

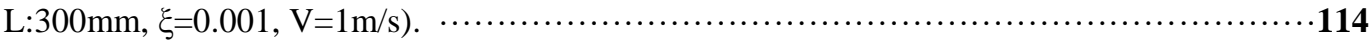

4.9 FE validation of the analytical model for the SS304 elastic plate (h:0.25mm, W: $35.35 \mathrm{~mm}$, $\mathrm{L}: 353 \mathrm{~mm}, \xi=0.001, \mathrm{~V}=1 \mathrm{~m} / \mathrm{s})$ 115

4.10 FE validation of the analytic model for the Al6061 elastic-plastic plate (h:0.88, W: 22.0, L:300, $\xi=0.001, \mathrm{~V}=1 \mathrm{~m} / \mathrm{s})$ 118

4.11 FE validation of the analytic model for the SS304 elastic-plastic plate (h:1.25, W: 35.35, L:353, $\xi=0.001, \mathrm{~V}=1 \mathrm{~m} / \mathrm{s})$. 
4.12 Post critical responses of the Al6061-T6 elastic plates of two different plate thicknesses under $\mathrm{V}=0.1 \mathrm{~m} / \mathrm{s}$ calculated from FEM and the analytical model: (a) $\mathrm{h}=0.1 \mathrm{~mm}$; (b) $\mathrm{h}=0.457 \mathrm{~mm} . \cdots 122$

5.1 Target core geometry of Al6061-T6 corrugated core sandwich columns and corresponding quasi-

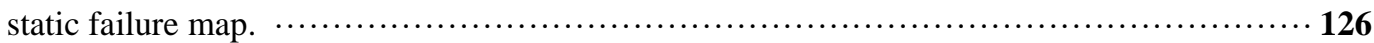

5.2 Target core geometry of SS304 corrugated core sandwich columns and corresponding quasi-

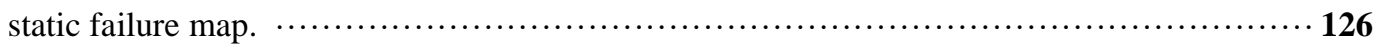

5.3 Dynamic effects on the boundary between elastic global buckling and face yield of Al6061-T6

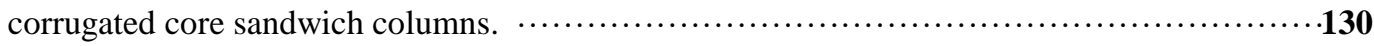

5.4 Dynamic effects on the boundary between elastic face wrinkling and face yield of Al6061-T6

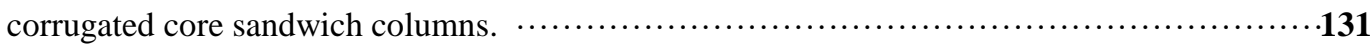

5.5 Dynamic effects on the change of failure modes from global buckling to local buckling. $\cdots \cdots 134$

5.6 Effect of imperfections on a failure map boundary of Al6061-T6 corrugated core sandwich

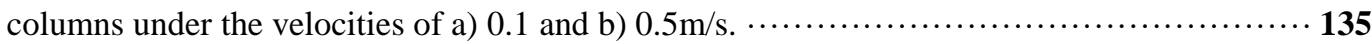

5.7 (a) Type I; (b) Type II; (c) Quasi-static load-deflection curve of Type I and Type II structures

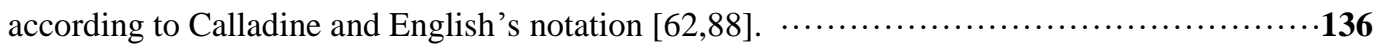

6.1 Weight comparison of corrugated core sandwich columns of $\mathrm{L}=1 \mathrm{~m}$ under quasi-static loading.

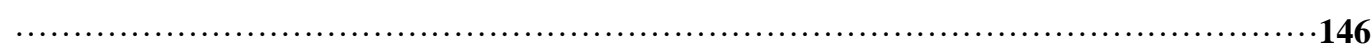

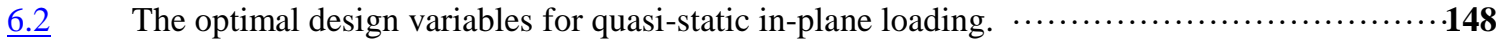

6.3 Competing failure modes of Al6061-T6 sandwich columns compressed perpendicular-to-

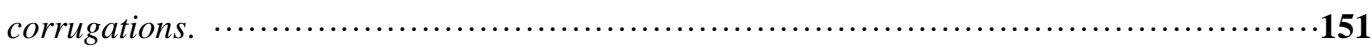

6.4 FE impulse capacity calculation of a sandwich column failed by local plate buckling.. 153

6.5 Competing failure modes of Al6061-T6 sandwich columns compressed parallel-to-corrugations under $\mathrm{V}=1 \mathrm{~m} / \mathrm{s}$. 154

6.6 Competing failure modes of SS304 sandwich columns compressed perpendicular-tocorrugations under (a) $\mathrm{V}=1 \mathrm{~m} / \mathrm{s}$ and (b) $\mathrm{V}=0.5 \mathrm{~m} / \mathrm{s}$.

6.7 Competing failure modes of SS304 sandwich columns compressed parallel-to-corrugations under $\mathrm{V}=1 \mathrm{~m} / \mathrm{s}$. 158

6.8 EGB (perpendicular-to-corrugations): (a) Residual plot and (b) Response surface for elastic

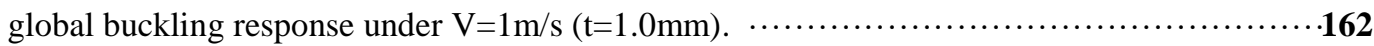

6.9 PGB (perpendicular-to-corrugations): (a) Residual plot and (b) Response surface for plastic

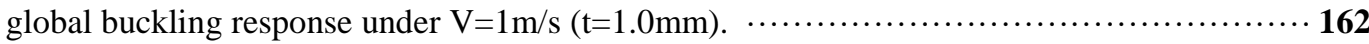

6.10 EFW (perpendicular-to-corrugations): (a) Residual plot, and (b) Response surface for elastic face wrinkling response under $\mathrm{V}=1 \mathrm{~m} / \mathrm{s}(\mathrm{t}=1.0 \mathrm{~mm})$. 
6.11 PFW (perpendicular-to-corrugations): (a) Residual plot and (b) Response surface for plastic face

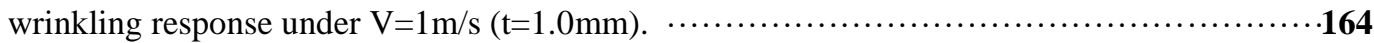

6.12 Comparisons of minimum weight designs of Al6061 corrugated core sandwich columns under (a)

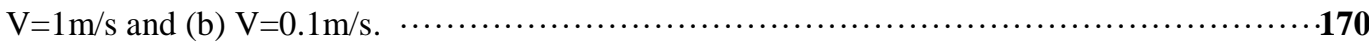

6.13 Variation of optimal core height for face sheet thickness h of optimal design for Al6061T6

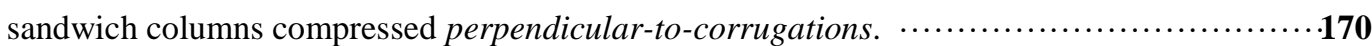

6.14 Local buckling strength, $\mathrm{h} / \mathrm{l}$ and $\mathrm{t} / \mathrm{l}$, for the same areal mass of Al6061T6 sandwich columns compressed parallel-to-corrugations under quasi-static loading, $\mathrm{V}=1 \mathrm{~m} / \mathrm{s}$ and $\mathrm{V}=0.1 \mathrm{~m} / \mathrm{s}$.

7.1 Typical reaction curve of corrugated core sandwich columns compressed perpendicular-to-

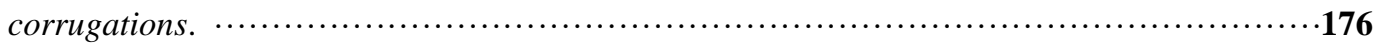

7.2 Deformation of the Al6061-T6 corrugated core sandwich columns of $h=2.2 \mathrm{~mm}, \mathrm{~L}=469 \mathrm{~mm}$,

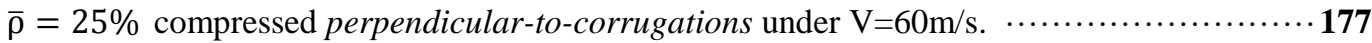

7.3 Back end reaction forces depending on applied velocities of $V=20,60,100 \mathrm{~m} / \mathrm{s} . \cdots \cdots \cdots \cdots \cdots \ldots \ldots$

7.4 Front end reaction forces depending on applied velocities of $\mathrm{V}=20,60,100 \mathrm{~m} / \mathrm{s} . \cdots \cdots \ldots \ldots \ldots \mathbf{1 7 8}$

7.5 Length dependence: (a) Front end reaction force; (b) Back end reaction force. ….......... 179

7.6 Global buckling mode (mode 7) imperfection sensitivity: (a) imposed eigenmode for the imperfection; (b) front end reaction force; (c) back end reaction force. …................. 180

7.7 Face wrinkling mode (mode 6) imperfection sensitivity: (a) front end reaction force; (b) back end reaction force.

181

7.8 Simplified model of the front and back end reaction force curves for corrugated core sandwich columns under high-velocity compression perpendicular-to-corrugations. 183

7.9 Analytic prediction of peak reaction forces of corrugated core sandwich columns under high velocity compression perpendicular-to-corrugations.

186

7.10 Core contribution to in-plane stiffness depending on the magnitude of compression velocity : (a) low velocity; (b) high velocity. 187

7.11 The comparison of reaction forces between rate-dependent FEA and rate-independent FEA: (a)

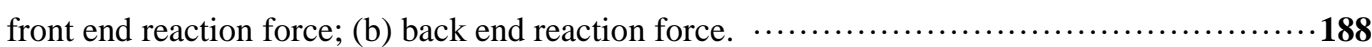

7.12 Comparison between R-scaled rate-independent analysis and the rate-dependent analysis, (a) front end reaction force; (b) back end reaction force. 189

7.13 Approximated $\sigma_{11}-\varepsilon_{11}$ diagram of an elastic-bilinear plastic material (SS304) in the presence of lateral expansion constraint.

190

7.14 Analytic prediction of peak reaction forces of rate-dependent (SS304) corrugated core sandwich columns under high velocity compression perpendicular-to-corrugations: (a) h=1.25mm; (b) $\mathrm{h}=2.5 \mathrm{~mm}$. 
7.15 Representative reaction force curves of a corrugated core sandwich column compressed parallelto-corrugations. The curves are regarding an Al6061-T6 sandwich column of $\mathrm{h}=2.2 \mathrm{~mm}$,

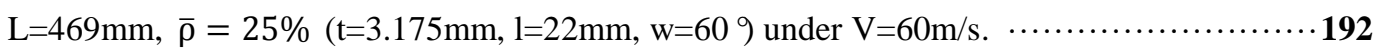

7.16 Wave propagation in the representative sandwich column presented in Fig.7.15. .......... 193

7.17 Velocity-dependent reaction forces at (a) the front end, (b) the back end. …............... 195

7.18 Effects of column length at $\mathrm{V}=60 \mathrm{~mm}$ : (a) front end reaction force; (b) back end reaction force. 195

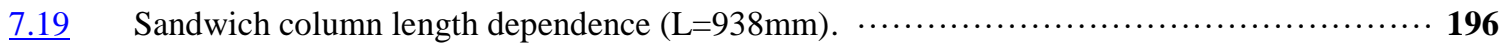

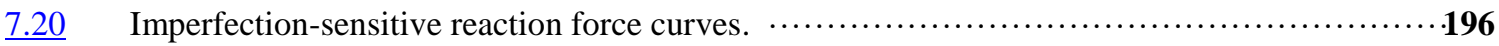

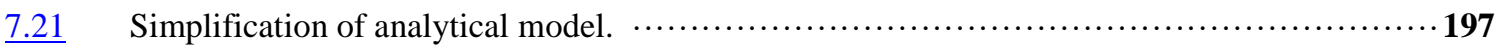

7.22 Comparison between analytic expressions and FE simulations of compression-velocity dependent peak reaction forces, $\mathrm{P}^{\text {(front) }}$ and $\mathrm{P}^{\text {(back) }}$, of Al6061-T6 sandwich columns having various face sheet thicknesses: (a) h=0.457mm; (b) h=2.2mm; (c) h=4.4mm............................ 199

7.23 Material strain-rate dependence of SS304 corrugated core sandwich columns compressed

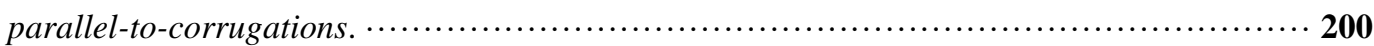

7.24 Three wave fronts propagated in a rate-independent elastic- plastic material with bilinear

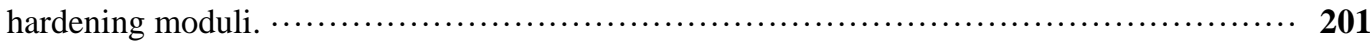

7.25 Comparison between the rate-dependent analysis and the scaled curves from the rate-independent

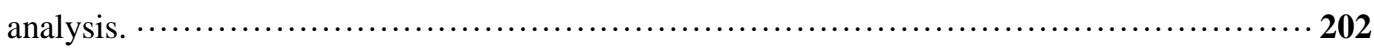

7.26 Analytic prediction of peak reaction forces of rate-dependent (SS304) corrugated core sandwich

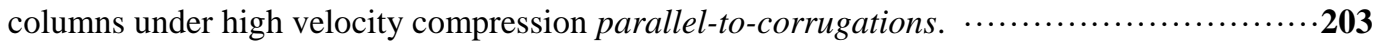

B1 Change of vertical position of a node due to in-plane loading: (a) Before in-plane compression; (b)

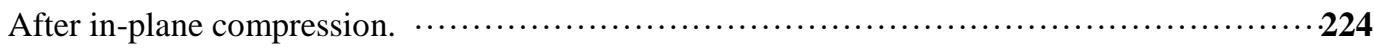

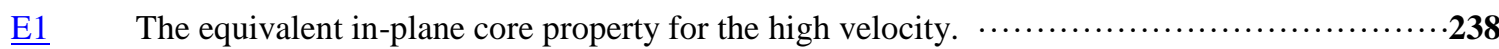




\section{List of Tables}

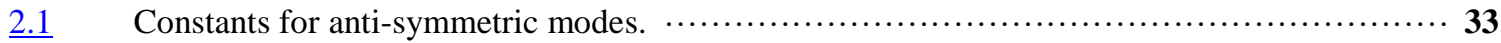

2.2 The geometric dimensions of columns for the global buckling analytical model

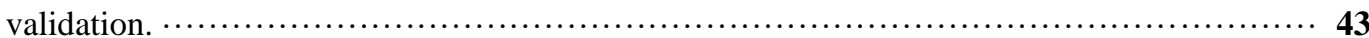

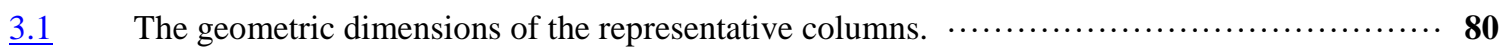

4.1 Geometric dimensions and velocity of FE sandwich column models for the investigation. ..... 97

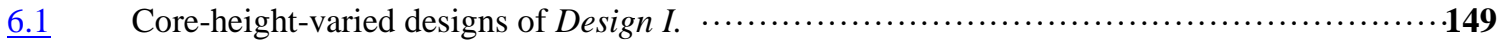

6.2 Core-height-varied designs of Design II. …

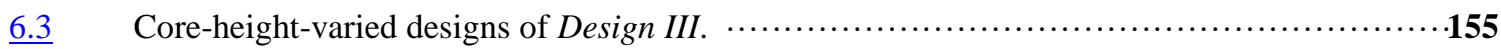

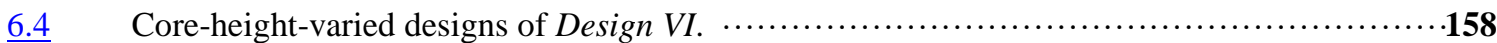

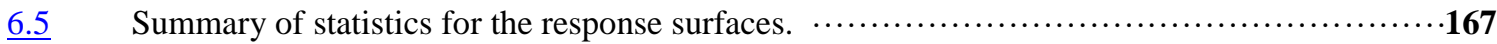

D1 Full Factorial experimental design for global buckling response of sandwich columns

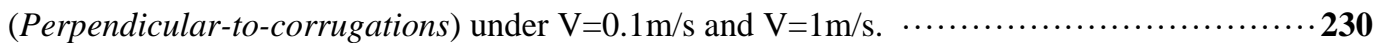

D2 Full Factorial experimental design for face wrinkling response of sandwich columns compressed

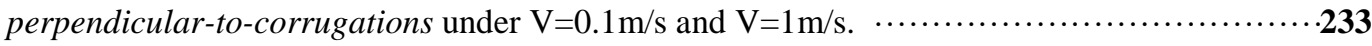

D3 Full factorial experimental design for global buckling response of sandwich columns compressed parallel-to-corrugations under $\mathrm{V}=0.1 \mathrm{~m} / \mathrm{s}$ and $\mathrm{V}=1 \mathrm{~m} / \mathrm{s}$.

236

D4 Full factorial experimental design for local plate buckling response of sandwich columns compressed parallel-to-corrugations under $\mathrm{V}=0.1 \mathrm{~m} / \mathrm{s}$ and $\mathrm{V}=1 \mathrm{~m} / \mathrm{s}$. 


\section{Nomenclature}

Superscript

(m)

(SW)

(perp)

(para)

Subscript

(el)

(pl)

$v$

$v_{\mathrm{P}}$

$\kappa$

$\omega$

$\bar{\rho}$

$\rho$

$\sigma$

$\sigma_{Y}$

$\varepsilon$

$\xi$

$\xi_{0}^{(L P)}$

$\xi_{0}^{(f)}$ monolithic solid column

corrugated core sandwich column

perpendicular-to-corrugations

parallel-to-corrugations

elastic

plastic

Poisson's ratio

plastic Poisson's ratio (In this thesis, $v_{\mathrm{P}}=0.5$ )

Becque's parmeter (the ratio of the plastic strain increment in the principal 2 direction to the plastic strain increment in the principal 1 direction)

corrugation angle

core relative density

parent material density

material stress

yield stress

material strain

the imperfection amplitude of global curvature

the imperfection amplitude of local plate buckling

the imperfection amplitude of face wrinkling 


\begin{tabular}{|c|c|}
\hline$\varphi^{(S W)}$ & rotation due to bending moments \\
\hline$\varphi$ & Becque's constant \\
\hline A & cross sectional material area \\
\hline $\mathrm{A}_{\text {eff }}$ & aerial mass, effective cross sectional material area \\
\hline $\mathrm{a}$ & the longitudinal length of a patch plate \\
\hline$\overline{\mathrm{a}}$ & $\begin{array}{l}\text { a nondimensional time parameter determining the rate of the growth of } \\
\text { the fundamental mode in an elastic monolithic solid column subject to a } \\
\text { constant load } \mathrm{P}\end{array}$ \\
\hline $\mathrm{b}$ & the width of a patch plate \\
\hline $\mathrm{c}$ & core height \\
\hline$c_{e l}$ & elastic wave speed \\
\hline$c_{e l}^{(m)}$ & elastic wave speed of monolithic solid column \\
\hline$c_{e l}^{(S W)}$ & elastic wave speed of corrugated core sandwich column \\
\hline$c_{e l}^{(p e r p)}$ & $\begin{array}{l}\text { elastic wave speed of corrugated core sandwich column compressed } \\
\text { perpendicular-to-corrugations }\end{array}$ \\
\hline$c_{e l}^{(\text {para })}$ & $\begin{array}{l}\text { elastic wave speed of corrugated core sandwich column compressed } \\
\text { parallel-to-corrugations }\end{array}$ \\
\hline$c_{p l}^{(m)}$ & elastic-plastic wave speed of monolithic solid column \\
\hline$c_{p l}^{(S W)}$ & elastic-plastic wave speed of corrugated core sandwich column \\
\hline$c_{p l}^{(p e r p)}$ & $\begin{array}{l}\text { elastic-plastic wave speed of corrugated core sandwich column } \\
\text { compressed perpendicular-to-corrugations }\end{array}$ \\
\hline$c_{p l}^{(\text {para })}$ & $\begin{array}{l}\text { elastic-plastic wave speed of corrugated core sandwich column } \\
\text { compressed parallel-to-corrugations }\end{array}$ \\
\hline $\mathrm{D}$ & total flexural rigidity of a sandwich column \\
\hline$D_{e l}^{(m)}$ & flexural rigidity of elastic monolithic solid column \\
\hline$D_{p l}^{(m)}$ & flexural rigidity of elastic-plastic monolithic solid column \\
\hline
\end{tabular}




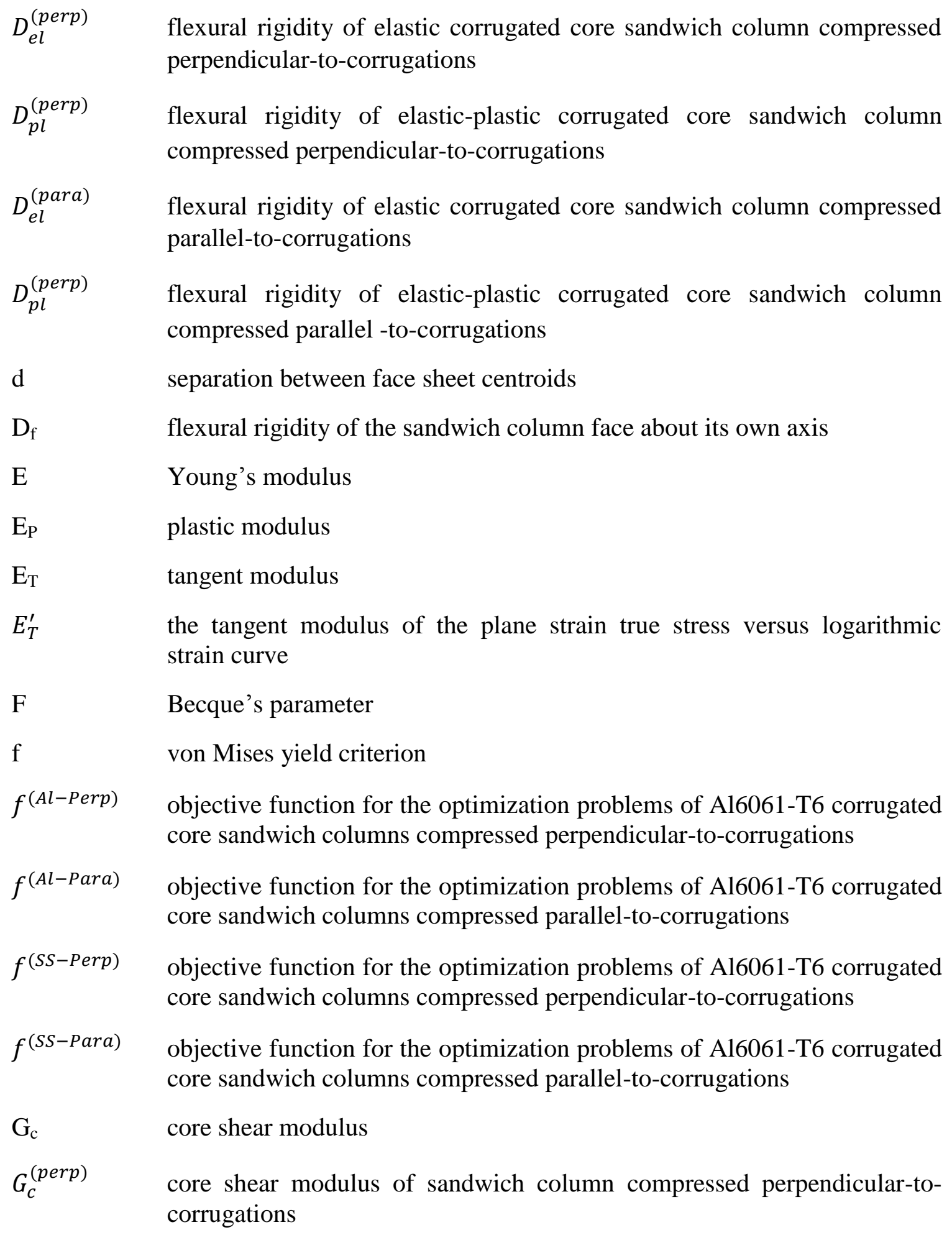




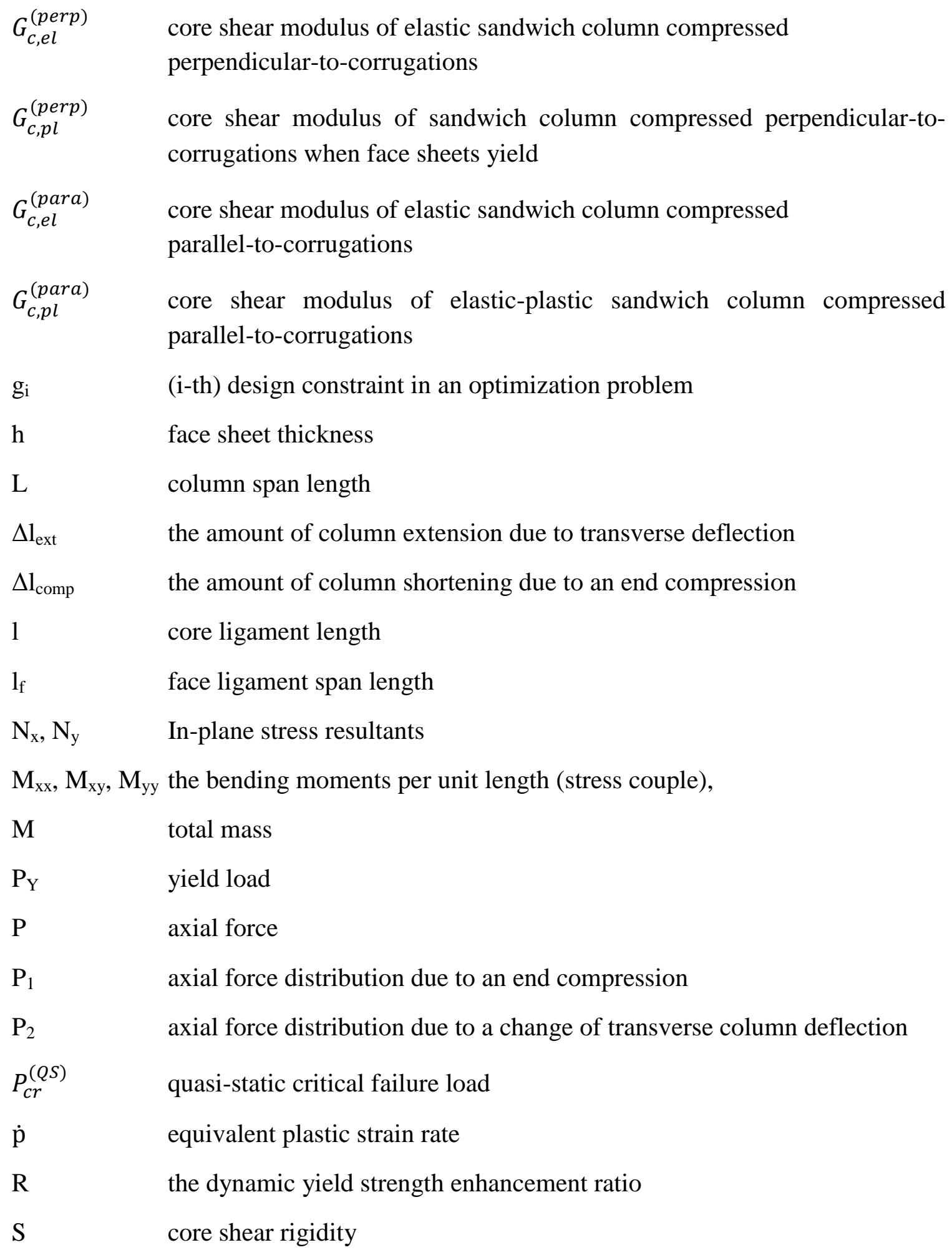


$S_{e l}^{(\text {perp })} \quad$ shear rigidity of elastic sandwich column compressed perpendicular-tocorrugations

$S_{p l}^{(p e r p)} \quad$ shear rigidity of elastic-plastic sandwich column compressed perpendicular-to-corrugations

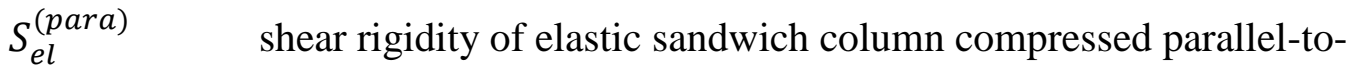
corrugations

$S_{p l}^{(p a r a)} \quad$ shear rigidity of elastic-plastic sandwich column compressed parallel-tocorrugations

$\mathrm{t}$

core ligament thickness

V

compression velocity, rate-of-compression

$\mathrm{w}_{0}$

global curvature imperfection

$w^{(m)}$

transverse displacement of monolithic solid column from the undeformed initial curved shape

$w^{(S W)} \quad$ transverse displacement of corrugated core sandwich column from the undeformed initial curved shape

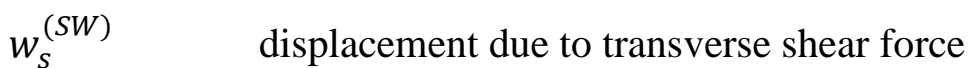

$\mathrm{W}(\mathrm{x}) \quad$ base functions (Eigen functions) for column deformation 


\section{CHAPTER 1}

\section{INTRODUCTION}

\subsection{MOTIVATION}

Sandwich structures, consisting of a lightweight core material separating two thin face sheets, have been widely used in various structural applications in which minimized weight is required. In addition, selection of a particular core material system makes it possible for them to perform multi-functional roles; a channel for fluid flow, an impact mitigator, a heat exchanger, etc. In particular, metallic core sandwich structures with periodicity have been given a great deal of attention because of their excellent mechanical properties and multifunctional capabilities, due to recent advances in manufacturing technology [1,2]. This dissertation focuses on one of metallic core sandwich structures, a corrugated core sandwich column.

In order to fully exploit such benefits, the mechanical behavior under various loading conditions should be understood. One loading scenario receiving significant attention is dynamic compression of metallic core sandwich structures. There has been significant effort to predict and quantify the structural performance of metallic core sandwich structures in this situation. Motivated by blast mitigation applications, the dynamic out-of-plane response of impulsively loaded metallic core sandwich panel structures has been actively studied for the last decade [3-24]. These studies are characterized by high magnitude loads and short duration times. Compression velocity range in these studies is on the order of tens of $\mathrm{m} / \mathrm{s}$ up to a few hundred $\mathrm{m} / \mathrm{s}$, which 
corresponds to that of a front face in a typical sandwich panel subject to underwater blast loads $[3,25]$. In air blast loading, the impulse is also characterized as impulsive with the same order of time scale $(0.1 \mathrm{~ms}$ duration) as in an underwater blast but with lower magnitude [20, 24]. However, the difference between two media is found in the amount of transferred impulse to the structures (fluid-solid interaction): the sandwich structures adopting a light top face sheet can benefit from reduction in transferred impulse to the structures in water, whereas the external pulse is fully absorbed by the structures in air $[20,24]$.

However, limited attention has been paid to the dynamic in-plane behavior of sandwich columns to date. Recently, Ji [26] considered a dynamic impact problem in which the sandwich column made of carbon/epoxy face sheets and a polyurethane foam core was subject to impact velocities of an order of $\mathrm{m} / \mathrm{s}$. However, no study on the metallic core sandwich columns in dynamic in-plane compression can be found as far as the author knows.

On the other hand, the quasi-static in-plane responses of pyramidal truss cores and corrugated prismatic cores sandwich columns were studied systematically by employing analytical, numerical, and experimental methods [27-29]. The influences of material properties, geometry, and fabrication methods, on the failure mechanisms exhibited in those columns were discussed. Consequently, minimized weight designs were achieved for the sandwich columns employing those metallic periodic cores.

Nevertheless, fundamental questions still need to be answered regarding the dynamic response of the metallic core sandwich columns. As the loading rate increases, complex dynamic effects become involved, thereby changing the response. This 
deficiency is addressed in this thesis.

\subsection{OBJECTIVES}

The main objective of this dissertation is to investigate dynamic effects on the structural response of corrugated core sandwich columns through theoretical and numerical analysis. Considering the highly anisotropic character of the corrugated core, two in-plane loading directions, perpendicular-to-corrugations and parallel-tocorrugations, are considered as shown in Fig. 1.1. In this dissertation, the investigation will concern compression velocities ranging from quasi-static loading up to $100 \mathrm{~m} / \mathrm{s}$, divided into low (up to the order of a few $\mathrm{m} / \mathrm{s}$ ) and high (the order of tens of $\mathrm{m} / \mathrm{s}$ ) velocity regimes.

In the low velocity response regime, dynamic effects on individual failure modes will be assessed. Depending on the overall column geometry, core design, and parent material properties, corrugated core sandwich columns fail in several ways: global buckling and local buckling. To this end, prediction methods are required for the individual failure modes for each in-plane loading orientation. Although finite element methods can be used to predict the dynamic response of these structures $[3-8,15-16,18$ 22-24], there are drawbacks. First, it is too time-consuming due to a repetitive core structure, which must be modeled with many elements. The other is that it cannot explain the competition of the individual failure modes even though it is a good predictor for a given column geometry and loading condition. Therefore, analytical models to predict the individual failure modes of sandwich columns under low velocity compression will be developed, and then validated by comparing with FE simulations. The effects of the rate- 


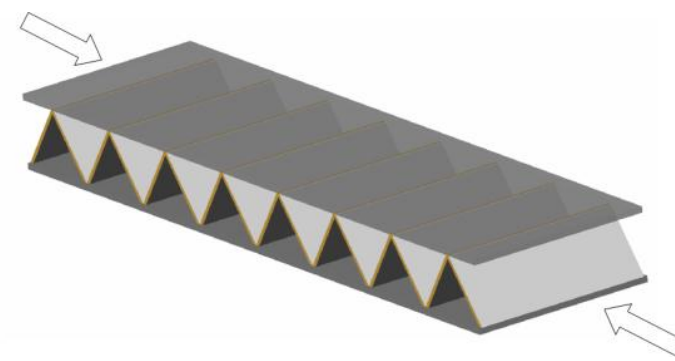

(a)

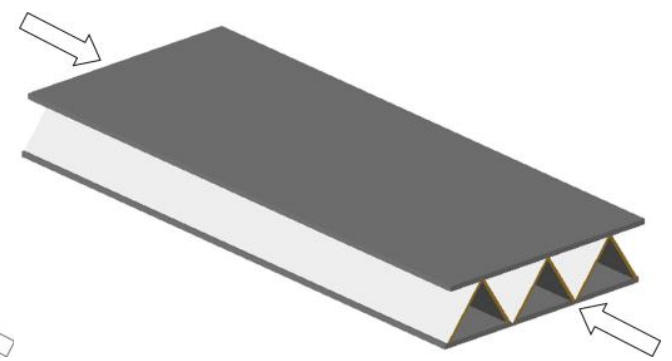

(b)

Figure 1.1: In-plane loading orientations: (a) Perpendicular-to-corrugations; (b) Parallel-tocorrugations.

of-loading on failure mechanism maps and minimum weight designs will be assessed. Additionally, a comparative study of monolithic and sandwich columns having equal mass will be performed to discuss the structural benefit of sandwich structures for dynamic applications.

With respect to the high velocity regime, the response time scale is expected to be different from that of the low velocity response. It is hypothesized that axial stress wave propagation during one-way or one round trip can explain the high velocity sandwich column response. The dynamic response of corrugated core sandwich columns in the high velocity regime is analytically characterized on the basis of FE observations.

\subsection{BACKGROUND}

\subsubsection{Dynamic Response of Metallic Core Sandwich Structures}

The dynamic in-plane response of metallic core sandwich columns has not received much attention. However, the dynamic out-of-plane response of metallic core sandwich structures subject to impulsive loading has been actively investigated for the last decade [3-24]. The primary purpose of the recent dynamic out-of-plane metallic core sandwich 
studies is to enhance blast protection performance by employing the above mentioned sandwich designs. Accordingly, efforts are made to investigate the sandwich structure deformation mechanisms and momentum transfer under impulsive loading and to identify the effects of metallic cores, specifically, the selection of core topology and relative core density, on the dynamic performance.

The dynamic response of the sandwich beams and rectangular sandwich plates with Y-frame and corrugated cores have been investigated using FEM and other experiments by several researchers [7-9]. In these studies, a metal foam projectile was projected transversely to the sandwich beams and plates with their ends clamped at initial velocities up to $500 \mathrm{~m} / \mathrm{s}$ (corresponding impulse of $7.0 \mathrm{kNs} . \mathrm{m}^{-2}$ ). In addition, analytical models have been developed to predict the out-of-plane response under impulsive loads [10,11].

Mori et al. carried out several tests on circular sandwich plates having an I-core, a honeycomb core, and a pyramidal truss core $[12,13]$. They employed a novel testing method, in which an impulsive water pressure was applied to the entire surface of the front face sheet and then measured transverse displacement using shadow Moiré. Wadley et al. [14] used a dynocrusher tester to investigate the dynamic compressive response of square honeycomb structures. Wei et al. [15] performed dynamic compression tests on multi-layered sandwich panels, and simulated the dynamic response via FEM. In parallel with the experimental studies, analytical and numerical studies of the underwater blast performance for optimal sandwich panels design have been performed by many researchers [16-24].

These and other studies continue to show that the out-of-plane dynamic performance is significantly impacted by the properties of metallic core. Therefore, 
efforts have been made to characterize various core topologies such as I-core, honeycomb, pyramidal truss and corrugated core, via FEA and gas gun tests [3-6]. Those studies investigated the core buckling motion and provided the expressions for front and back end stresses with respect to the imposed velocity and relative core density under dynamic out-of-plane compression. Thus, the adequate selection of core has been realized by understanding the dynamic response and energy dissipation mechanism in core compression through these studies.

However, research into the dynamic column response of metallic core sandwich structures is very limited.

\subsubsection{Dynamic Analysis of Monolithic Solid Columns}

In order to establish a theoretical basis for the dynamic sandwich column response, we must review previous studies on monolithic solid columns. Research regarding the dynamic buckling of monolithic solid columns has a long history where various boundary types, velocity ranges and geometries have been considered. According to Lindberg [30], Zhang [31] and Simitses [32], dynamic stability research can be classified by the characteristics of applied loading: vibration buckling by an oscillatory load and pulse buckling induced by a single pulse. More specifically, the pulse can be categorized as 1) an impulsive pulse with short duration and 2) a step pulse with long duration and constant magnitude. Again, the step-pulse dynamic problem has three subclasses depending on the type of loading condition: (2a) a constant load problem, (2b) a constant velocity problem and $(2 \mathrm{c})$ a constant mass problem.

A constant load problem of simply supported elastic columns was investigated by 
Jones [33]. An important finding was reported that the beam oscillates transversely if the applied load was less than the Euler buckling load $\mathrm{P}_{\mathrm{E}}$; otherwise the amplitude in transverse deflection grows exponentially. The Jones' unimodal approach was extended by Lindberg and Florence [30], in which higher buckling modes were included. In addition, Lindberg [34] proposed a predominant buckling mode depending on the magnitude of applied load for a constant load problem. Recently, a constant load problem considering the effect of wave propagation was solved semi-analytically by Lepik [3537].

A constant velocity problem where one end of an elastic column was compressed at a slow constant rate was considered by Hoff [38]. Findings similar to Jones' [33] were seen in this study. Without considering a nonlinear strain term in the equations of motion, modal amplitude in the transverse motion of column is given by a combination of Bessel functions (bounded) as long as the axial force is lower than the Euler buckling load, which changes into the modified Bessel functions (unbounded) if axial force exceeds the Euler buckling load. However, in cases where a large transverse deflection occurs (i.e. the response past a maximum peak load), a power series solution is appropriate - rather than the analytical solution-because the nonlinear strain term can be no longer ignored. Sevin [39] extended Hoff's study [38] by adding the axial inertia effect of a simply supported elastic column. Together with the analytical expressions for beam deflection and non-uniform axial stress distribution, an additional description of lateral motion was also given by the Finite Difference Method (FDM). Using the closed-form analytical solutions and the FDM results, he concluded that axial inertia effect caused by geometric nonlinearity could be negligible compared with the axial inertia effect caused by end 
compression until the axial force reaches a maximum peak [39]. For the dynamic instability of inelastic columns in a constant velocity problem, Lee [40-42] also proposed a quasi-bifurcation criterion.

Considering the constant mass impact problems of elastic columns, Ari-Gur et al. $[43,44]$ carried out a combined numerical and experimental investigation. It was verified that high slenderness ratio, small imperfection magnitude, and short contact duration produced a high Dynamic Load Factor (DLF), which is defined as a ratio of dynamic peak load to the static Euler buckling load. Hayashi and Sano $[45,46]$ also considered dynamic elastic buckling problems with impact boundary conditions. The FDM results showed the development of higher buckling modes, highlighting the influence of initial velocities on axial inertia, and the local deformation near the end of impact. The effects of shear and rotary inertia were also investigated even though these proved not to be significant. Recently, Ji and Wass [26,47] considered a constant mass impact problem of slender beams. Assuming a perfectly straight beam, the travelling elastic waves were included in the lateral equation of motion, and the time-to-buckle, and the mode shapes were derived by finding the dynamic bifurcation buckling state. Kenny et al. performed a numerical and experimental study on a constant mass problem taking the traveling waves into account $[48,49]$.

The dynamic plastic buckling problem in which high compression velocities are involved, have been investigated by several researchers. Abrahamson and Goodier [50] carried out pioneering experimental work on the dynamic plastic buckling problem where aluminum rods impacted a rigid target at high velocities producing plastic strain. In their consideration of dynamic plastic buckling, a theoretical half-wave length was suggested 
by a predominant buckling mode.

$$
L_{c r}=\pi r\left(2 E_{T} / \sigma\right)
$$

where $\mathrm{r}$ is the radius of gyration, $\mathrm{E}_{\mathrm{T}}$ is the tangent modulus, and $\sigma$ is stress. Vaughn and Hutchinson [25,51] studied the interaction between axial waves and bucklewaves for a stocky column subject to a high rate of compression, and concluded that these were highly interactive if a dimensionless parameter, $V /\left(c_{e l} \varepsilon_{Y}\right)$, a function of applied velocity, $\mathrm{V}$, elastic stress wave speed, $\mathrm{c}_{\mathrm{el}}$, and yield strain, $\varepsilon_{\mathrm{Y}}$, was greater than five. For this intense loading situation, they set up a theoretical problem for the incident plastic stress wave region within the column and solved the governing equation with a timevarying boundary condition using the Galerkin method. On the other hand, Bell [52] proposed a critical wavelength $\mathrm{L}_{\mathrm{cr}}$ for an inelastic column based on the modified EulerEngesser theory and compared with his experimental data.

$$
L_{c r}=\frac{n \pi r}{k} \sqrt{\frac{E_{T}}{\sigma_{\max }}}
$$

where $\mathrm{n}$ is mode number, $\mathrm{k}$ is a constant associated with the end condition.

Material rate effects on dynamic column buckling problem of slender beams have been assessed through FDM by Sugiura et al. [53]. They concluded that the rate dependence affects only post-dynamic instability within the considered velocity range of $0.02-0.25 \mathrm{~m} / \mathrm{s}$.

At an intermediate-velocity axial impact load, which simulates fluid-solid axial slamming around several m/s (Impulse : $1900 \mathrm{~kg}, \mathrm{~m} / \mathrm{s}$, System mass: $453 \mathrm{~kg}$ ), Cui and his coworkers [54-59] and Zhang [31] separately conducted dynamic column buckling experiments. They systematically investigated the effects of imperfections, slenderness 
ratio, and pulse duration by the analytical and numerical methods. Several failure criteria such as dynamic critical buckling criterion, dynamic critical plasticity criterion, and dynamic collapse criterion, were proposed and evaluated through a series of test columns.

\subsection{DISSERTATION OUTLINE}

This dissertation consists of three parts: The first two parts focus on the compression velocity range from quasi-static loading to an order of a few $\mathrm{m} / \mathrm{s}$ and the last part is associated with high velocity response under a compressive velocity of tens of m/s.

In Part I (Chapters 2-4), analytical models are developed. There is a separate chapter dedicated to each of the individual modes in the low velocity region. In Chapter 2, an analytical model for global buckling response is developed. The global buckling analytical model is not only applicable to corrugated core sandwich columns in compression with constant rates in the two in-plane directions but also to monolithic solid columns. The analytical results are compared with FE calculations for the columns made of A16061-T6 and SS304 under several compression velocities, V=0.1 5m/s. The details about the FE simulations contained in the remaining chapters are given there. In Chapter 3, the analytical model for face wrinkling response of sandwich columns loaded perpendicular-to-corrugations is developed. One of the primary assumptions is verified by comparing the apparent elastic stress wave speeds of corrugated core sandwich columns loaded perpendicular-to-corrugations with FEM. The model is validated by comparing reaction forces, time-to-failure with FE calculations. The effects of compression velocity and imperfections are also investigated both analytically and numerically. In Chapter 4, a theoretical approach for local plate buckling of sandwich 
columns compressed parallel-to-corrugations is discussed. First, local plate buckling failure of sandwich columns is observed by FE simulations. A theoretical model for long plates, corresponding to corrugated core plates or face plates, is developed. This model is also verified with FE simulations, and its limitations are discussed.

Chapters 5 and 6 focus on the investigation of competing failure mechanisms and optimal solutions under dynamic loading in the low velocity region. Chapter 5 addresses the dynamic effects of failure mechanism maps. The movement of boundaries of an Al6061-T6 quasi-static failure mechanism map is shown using the analytical models. In other words, the influences of rate-of-loading and imperfections on each boundary are revealed. The transition of failure modes due to increased rate-of-loading, from global to local buckling failure, is explained by probing a geometric point on an SS304 failure mechanism map. Chapter 6 discusses dynamic optimal solutions. First, quasi-static optimal solutions for A16061-T6 and SS304 corrugated core sandwich columns are given as references. To show how the minimum weight designs evolve as dynamic effects become more influential, maximum sustainable impulse capacities are calculated for the core-height-varied designs whose core heights are varied from the quasi-static optimal solutions. As an additional way of demonstrating the dynamic effects on the optimal solution, dynamic optimal solutions for Al6061-T6 corrugated core sandwich columns compressed perpendicular-to-corrugations at $\mathrm{V}=0.1 \mathrm{~m} / \mathrm{s}$ and $1 \mathrm{~m} / \mathrm{s}$ are obtained. The implicit relations between maximum sustainable impulse capacity and sandwich geometric parameters are given by employing a response surface methodology, and dynamic optimization problems are solved by an optimization algorithm, sequential quadratic programing $(\mathrm{SQP})$. 
In the last part, Chapter 7 presents the high velocity response characterization of corrugated core sandwich columns based on the FE results.

A summary of this dissertation along with suggestions for future work is given in Chapter 8. 


\section{CHAPTER 2}

\section{AN ANALYTICAL MODEL}

\section{FOR THE PREDICTION OF GLOBAL BUCKLING}

\subsection{INTRODUCTION}

This chapter is concerned with global buckling failure in corrugated core sandwich columns under dynamic in-plane compression.

Little attention has been given to the dynamic in-plane response of sandwich columns, especially under suddenly applied loads that may be encountered in real-life structural applications [26]. Furthermore, there is a lack of dynamic studies on metallic core sandwich columns.

In contrast, the dynamic problem of a monolithic column has a relatively long history and many theoretical and numerical studies have been conducted [30, 33-43, 45, $46,51,56,60-63]$. An analytical model for the prediction of dynamic global buckling of corrugated core sandwich columns is developed from knowledge derived from studies on monolithic solid columns.

Regarding the monolithic column studies, finding a closed-form solution is challenging due to the complexity of the governing equations. Approximate solutions have been sought: simplified structural models [60-62], conversion to a spring-mass system [63], modal approach [30, 33, 36, 41, 47], and finite difference method (FDM) $[39,43,45,46]$. The dynamic problem of monolithic columns can be divided into three classes depending on loading conditions: constant velocity problems, constant load 
problems and constant mass problems [30-32]. Of particular interest is a study presented by Hoff [38], where he considered a simply supported column under a constant velocity load. He presented both a power series solution and a solution in explicit form using a combination of Bessel functions and modified Bessel functions, disregarding the nonlinear strain term. It is noted that the buckling motion of a simply supported elastic column grows rapidly if axial force exceeds the Euler buckling load, $\mathrm{P}_{\mathrm{E}}$. An extension of the Hoff's study using FDM was published, in which the effect of axial inertia were taken into account by Sevin [39].

The objective in this chapter is to develop analytical models predicting dynamic global buckling failure in monolithic and corrugated core sandwich columns, which are able to assess the effects of sandwich geometric parameters, inertia, and initial curvature imperfections. The models will be validated by comparison with the Finite Element Method (FEM) through some examples.

The chapter is organized as follows. In Section 2.2, the problem is mathematically formulated. In Section 2.3, a method to estimate non-uniform axial force distribution, included in the formulated governing equations, is introduced via stress wave propagation theory. In Section 2.4, the analytical models for monolithic and sandwich columns are elaborated. In Sections 2.5 and 2.6, FE simulations are carried out in order to validate the analytical models. 


\subsection{PROBELM STATEMENTS}

\subsubsection{Problem Definition}

Three column structures are studied: compression of a monolithic solid column and compression of a corrugated core sandwich columns in two in-plane loading directions, as illustrated in Fig. 2.1. For all of these problems, one end (front end) is axially displaced at a constant speed of $\mathrm{V}$ with the other degrees of freedom (DOFs) constrained while the other end (back end) is completely fixed. The column material is either A16061-T6 or SS304, which can be modeled as a rate-independent elastic-plastic material and a rate-dependent one, respectively. All of the columns are assumed to have initial curvature imperfections in the form of the fundamental static buckling mode,

(a)
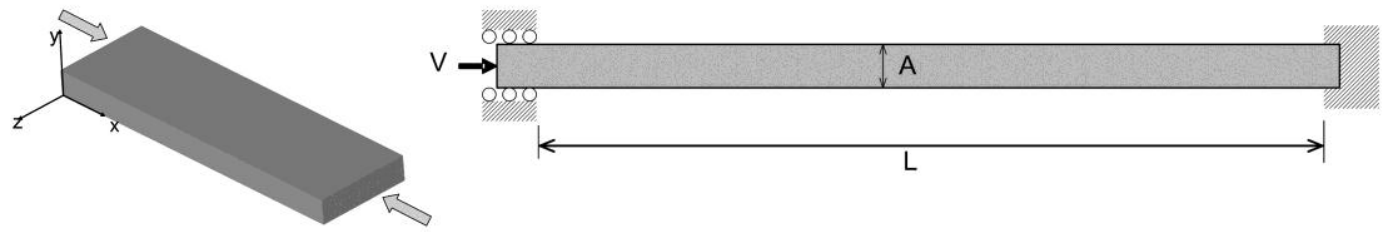

(b)
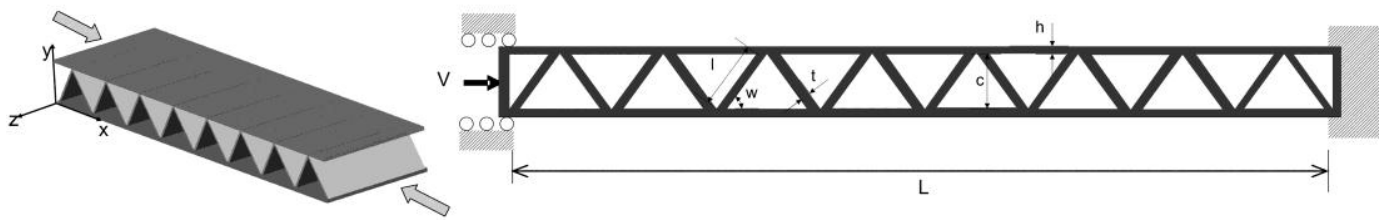

(c)
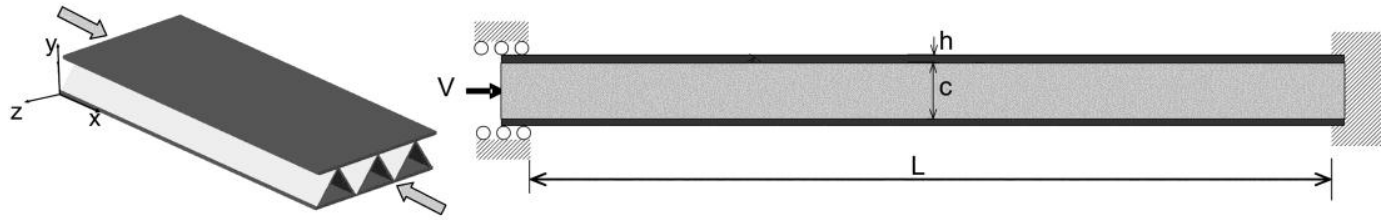

Figure 2.1: Three types of dynamic problems: (a) monolithic solid column; (b) corrugated core sandwich column compressed perpendicular-to-corrugations; (c) corrugated core sandwich column compressed parallel-to-corrugations. 
$w_{0}(x)=\frac{\xi}{2}\left(1-\cos \left(\frac{2 \pi x}{L}\right)\right)$

In this chapter, the considered range of applied velocity, $\mathrm{V}$, is $\frac{V}{c_{e l} \epsilon_{Y}}<1$ such that the incident stress wave at the moment of imposing compression is purely elastic. In the dimensionless parameter characterizing the intensity of the compressive loading, $\frac{V}{c_{e l} \epsilon_{Y}}$, $\mathrm{c}_{\mathrm{el}}$ and $\epsilon_{Y}$ denote the elastic wave speed and the strain at yielding, respectively, both of which are material properties in the case of a simple column. Consequently, the imposed velocity applied to the monolithic solid column problem covers up to $5 \sim 20 \mathrm{~m} / \mathrm{s}$ for many steel and aluminum alloys [25]. On the other hand, the elastic stress wave speed of sandwich columns, $c_{e l}^{(S W)}$, depends on sandwich geometries as well as materials, which will be discussed subsequently. Therefore, the compression velocity range for the sandwich problems differs from the one in the monolithic solid column problem (i.e., $5 \sim 20 \mathrm{~m} / \mathrm{s}$ ), but is approximately of the same order.

The sandwich parameters of a corrugated core sandwich column are indicated in Fig. 2.1(b) and (c). A face sheet is characterized by face sheet thickness, $h$, and face ligament span length, $2 \ell \cdot \cos \omega$. The global sandwich column geometry is characterized by column length, L, core height, $\mathrm{c}$, and core relative density, $\bar{\rho}$. Due to employed manufacturing methods, the geometries of Al6061-T6 and SS304 corrugated cores differ slightly [28]; the former is made using an extrusion/friction stir weld technique, and the latter is manufactured using a bending/brazing method. Therefore, the core relative density, $\bar{\rho}$, which is the ratio of core density to the density of parent material of which the column is made, is dependent on the manufacturing methods [28] and can be calculated using Eqns. (2.1) and (2.2). 


$$
\begin{aligned}
& \bar{\rho}_{A l}=\frac{t / \sin \omega}{\ell \cos \omega+t / \sin \omega} \\
& \bar{\rho}_{S S 304}=\frac{2 t}{\ell \sin 2 \omega}
\end{aligned}
$$

In Eqns. (2.1) and (2.2), $\ell, \omega, \mathrm{t}$ denote the length of corrugation leg, corrugation angle, and core ligament thickness, respectively.

For a comparative study against the corrugated core sandwich column, the problem for monolithic solid columns is established as described in Fig. 2.1(a).

\subsubsection{Formulation for Monolithic Solid Columns}

First, consider a monolithic solid column of thickness, A, column length, L, density, $\rho$, flexural rigidity, $\mathrm{D}^{(\mathrm{m})}$, with an initial curvature, $\mathrm{w}_{0}(\mathrm{x})$, compressed at a constant compression rate, V, as depicted in Fig. 2.2.

The equations of motion with boundary and initial conditions are given in Eqns. (2.3)-(2.5) [39].

$$
\begin{gathered}
\frac{\partial P(x, t)}{\partial x}=\rho A \frac{\partial^{2} u^{(m)}}{\partial t^{2}} \\
\frac{\partial^{2}}{\partial x^{2}}\left(D^{(m)} \frac{\partial^{2} w^{(m)}}{\partial x^{2}}\right)+\frac{\partial}{\partial x}\left(P(x, t) \frac{\partial\left(w^{(m)}+w_{0}^{(m)}\right)}{\partial x}\right)+\rho A \frac{\partial^{2} w^{(m)}}{\partial t^{2}}=0 \\
u^{(m)}(0, t)=V t, \quad u^{(m)}(L, t)=0 \\
w^{(m)}(0, t)=0, \quad \frac{\partial w^{(m)}}{\partial x}(0, t)=0 \\
w^{(m)}(L, t)=0, \quad \frac{\partial w^{(m)}}{\partial x}(L, t)=0
\end{gathered}
$$


In the above equations, $u^{(m)}(x, t)$ represents an axial displacement in the $x$ direction, $w^{(m)}(x, t)$ is a transverse displacement from the initial undeformed curved shape, $w_{0}^{(m)}(x, t)$, and $\mathrm{P}(\mathrm{x}, \mathrm{t})$ denotes an axial force distribution in the column length domain $[0, \mathrm{~L}]$, positive in compression. The flexural rigidity of the monolithic solid column, $\mathrm{D}^{(\mathrm{m})}$, is given in terms of material properties and thickness:

$$
\begin{aligned}
& D_{e l}^{(m)}=\frac{E A^{3}}{12\left(1-v^{2}\right)} \quad \text { if elastic, } \\
& D_{p l}^{(m)}=\frac{E_{T}^{\prime} A^{3}}{12} \quad \text { if elastic-plastic, }
\end{aligned}
$$

In Eqn. (2.6), $E_{T}^{\prime}$ is the tangent modulus of the plane strain true stress versus logarithmic strain curve of the parent material in the presence of lateral constraint due to a large dimension of the width of plates. The elastic-plastic flexural rigidity, $D_{p l}^{(m)}$, is obtained using the tangent modulus assumption of Shanley [64].

Eqn. (2.4), based on the Euler-Bernoulli assumption, is coupled with Eqn. (2.3) by $\mathrm{P}(\mathrm{x}, \mathrm{t})$. As the loading gets more intense, axial stress wave propagation effects become pronounced, which means that $\mathrm{P}(\mathrm{x}, \mathrm{t})$ cannot be assumed uniform over the column length, and thus, the equations get more complicated.

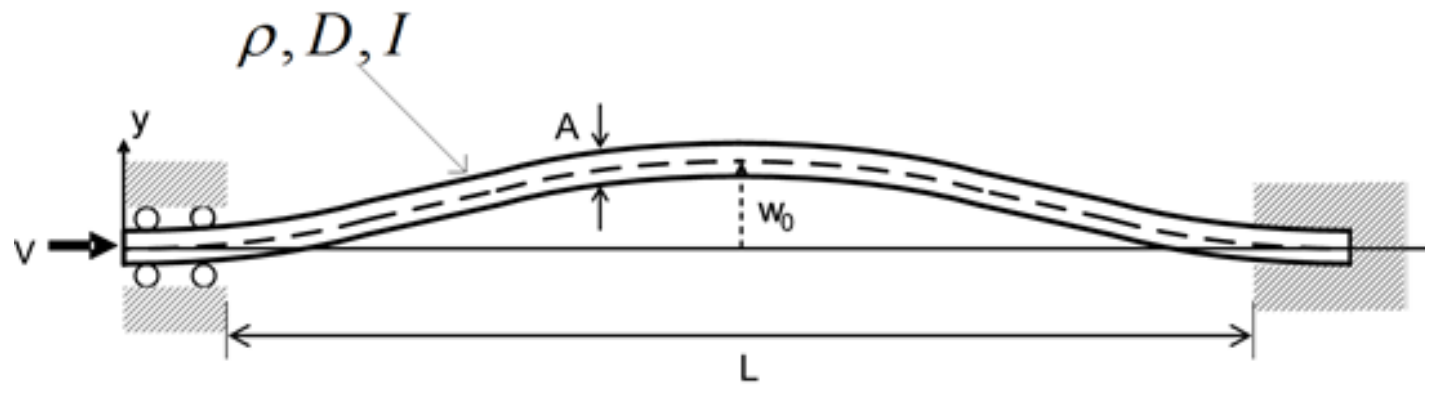

Figure 2.2: Configuration of the monolithic solid column problem. 


\subsubsection{Formulation for Corrugated Core Sandwich Columns}

Consider a corrugated core sandwich column of column length L and thickness $A=(c+2 h)$. Details of the sandwich parameters are indicated in Figs. 2.1 (b) and (c). The column is assumed to have an initial curvature imperfection in a form of the lowest static buckling mode, $w_{0}(x)=\frac{\xi}{2}\left(1-\cos \left(\frac{2 \pi x}{L}\right)\right)$. Including the effects of core shear deformation, the sandwich column can be simply taken as a structure with transverse inertia, $\rho \mathrm{A}^{(S W)}$, flexural rigidity, $\mathrm{D}^{(\mathrm{SW})}$, and shear rigidity, $\mathrm{S}^{(\mathrm{SW})}$. The superscript $(\mathrm{SW})$ is replaced by either (Para) or (Perp) to indicate the in-plane loading directions on sandwich columns; Parallel-to-corrugations and Perpendicular-to-corrugations. The equations of motion for axial, transverse and rotational directions are given by [46]

$$
\begin{gathered}
\frac{\partial P(x, t)}{\partial x}=(\rho A)^{(S W)} \frac{\partial^{2} u^{(S W)}}{\partial t^{2}} \\
(\rho A)^{(S W)} \frac{\partial^{2} w^{(S W)}}{\partial t^{2}}=\frac{d}{d x}\left(S^{(S W)} \cdot \frac{\partial w_{s}^{(S W)}}{\partial x}\right)-\frac{d}{d x}\left((P) \cdot \frac{\partial\left(w^{(S W)}+w_{0}^{(S W)}\right)}{\partial x}\right) \\
(\rho I)^{(S W)} \frac{\partial^{2} \varphi^{(S W)}}{\partial t^{2}}=\left(S^{(S W)} \cdot \frac{\partial w_{S}^{(S W)}}{\partial x}\right)+\frac{d}{d x}\left(D^{(S W)} \frac{\partial \varphi^{(S W)}}{\partial x}\right)
\end{gathered}
$$

where $\mathrm{u}, \mathrm{w}, \varphi$ are the axial, transverse displacement and rotation, respectively, and $\mathrm{S}^{(\mathrm{SW})}$

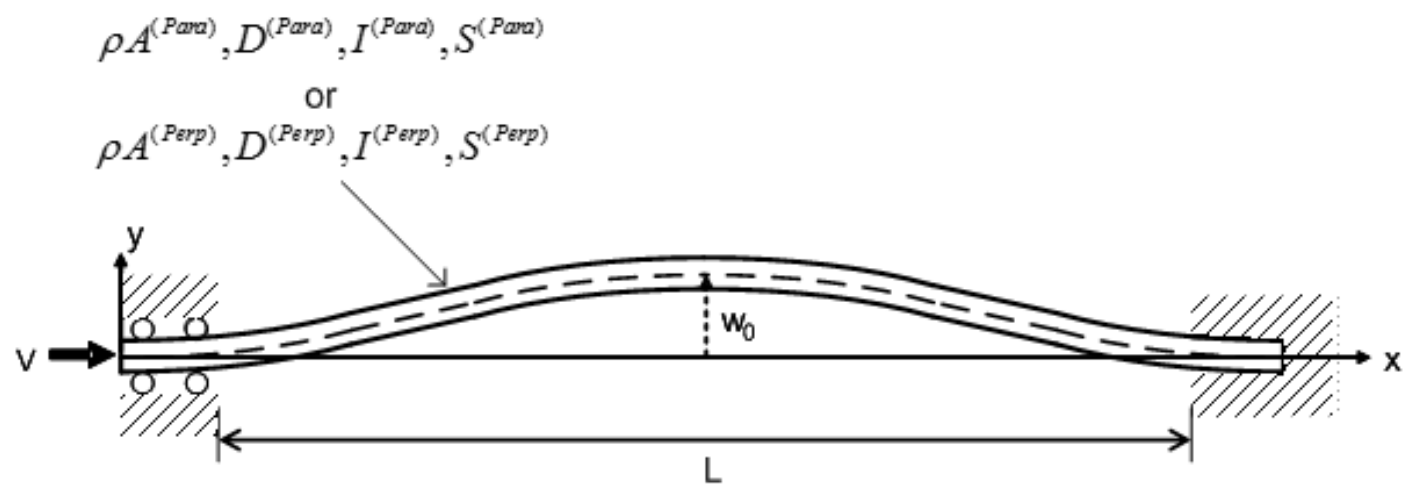

Figure 2.3: Configuration of the corrugated core sandwich column problem. 
and $\mathrm{D}^{(\mathrm{SW})}$ are the shear rigidity and flexural rigidity of sandwich columns. Taking core shear deformation into account, the transverse displacement, $\mathrm{w}^{(\mathrm{SW})}$, can be separated into deformations due to bending moments and transverse shear forces, $\varphi^{(\mathrm{SW})}$ and $\mathrm{w}_{\mathrm{s}}{ }^{(\mathrm{SW})}[65-$ 67].

$$
\frac{\partial w^{(S W)}}{\partial x}=\frac{\partial w_{S}^{(S W)}}{\partial x}+\varphi^{(S W)}
$$

Neglecting rotary inertia and eliminating $\mathrm{w}_{\mathrm{s}}{ }^{(\mathrm{SW})}$ via Eqn. (2.10), the governing equations are reduced to Eqn. (2.11) in which the effect of core shear deformation, $\mathrm{w}_{\mathrm{s}}{ }^{(\mathrm{SW})}$, is additionally included compared with Eqn. (2.4): Eqn. (2.11) can be reduced to Eqn. (2.4) if $S^{(S W)}$ goes to infinity.

$$
\begin{aligned}
& (\rho A)^{(S W)} \frac{\partial^{2} w^{(S W)}}{\partial t^{2}}+\frac{d}{d x}\left(P \cdot \frac{\partial w^{(S W)}}{\partial x}\right)+\frac{d^{2}}{d x^{2}}\left(D^{(S W)} \frac{\partial^{2} w^{(S W)}}{\partial x^{2}}\right)-\frac{d^{2}}{d x^{2}}\left(\frac{D^{(S W)} \rho A^{(S W)}}{S^{(S W)}} \frac{\partial^{2} w}{\partial t^{2}}\right) \\
& -\frac{d^{2}}{d x^{2}}\left(\frac{D^{(S W)}}{S^{(S W)}} \cdot \frac{d}{d x}\left[P \frac{\partial w^{(S W)}}{\partial x}\right]\right)=-\frac{d}{d x}\left(P^{(S W)} \cdot \frac{\partial w_{0}^{(S W)}}{\partial x}\right)+\frac{d^{2}}{d x^{2}}\left(\frac{D^{(S W)}}{S^{(S W)}} \cdot \frac{d}{d x}\left[P \frac{\partial w_{0}^{(S W)}}{\partial x}\right]\right)
\end{aligned}
$$

Since the corrugated cores show substantial anisotropy with respect to the in-plane loading directions, the flexural rigidity and shear rigidity are dependent on each in-plane loading direction.

In the in-plane loading direction of perpendicular-to-corrugation, the flexural rigidity of the sandwich column results mostly from the contribution of in-plane stiffness of face sheets.

$$
D_{e l}^{(p e r p)}=\frac{E}{\left(1-v^{2}\right)}\left[\frac{h(c+h)^{2}}{2}+\frac{1}{6} h^{3}\right]
$$




$$
D_{p l}^{(p e r p)}=E_{T}^{\prime}\left[\frac{h(c+h)^{2}}{2}+\frac{1}{6} h^{3}\right]
$$

On the other hand, shear is carried by the stretch and axial compression of the core [68]. According to Côté [68], the homogenized shear modulus of the core section is given by Eqn. (2.13). The shear rigidity, $S^{(\text {perp) }}$, of the sandwich column is assumed to be set by the shear rigidity of the corrugated core only, disregarding the shear stiffness of the faces as given by Eqn. (2.14) [28]. Moreover, the homogenized core shear modulus, even though the face sheets yield, is obtained by assuming that the core remains elastic since the major deformation mode of the corrugated core is folding at nodes between face sheets and corrugated cores.

$$
\begin{gathered}
G_{C}^{(\text {perp })}=\frac{E}{1-v^{2}} \frac{\bar{\rho}}{4} \sin ^{2} 2 \omega \\
S_{e l}^{(\text {perp })}=S_{p l}^{(\text {perp })}=A_{e f f} \cdot G_{C}^{(\text {perp })}=c \cdot \frac{E}{1-v^{2}} \frac{\bar{\rho}}{4} \sin ^{2} 2 \omega
\end{gathered}
$$

The major difference between the two in-plane loading directions, parallel-tocorrugations and perpendicular-to-corrugations, is that the corrugated core plates in the loading direction of parallel-to-corrugations participate in supporting a part of an axial compressive load. Therefore, the elastic flexural rigidity is given by

$$
D_{e l}^{(\text {para })}=\frac{E}{\left(1-v^{2}\right)}\left[\frac{h(c+h)^{2}}{2}+\frac{1}{6} h^{3}\right]+\frac{E \bar{\rho}}{12} c^{3}
$$

And in plastic region,

$$
D_{P l}^{(\text {para })}=E_{T}^{\prime}\left[\frac{h(c+h)^{2}}{2}+\frac{1}{6} h^{3}\right]+\frac{E_{T} \bar{\rho}}{12} c^{3}
$$


Elastic shear rigidity is given by [68]

$$
\begin{array}{r}
G_{C, e l}^{(\text {para })}=\frac{E}{2(1+v)} \bar{\rho} \sin ^{2} \omega \\
S_{e l}^{(\text {Para })}=A_{e f f} \cdot G_{C, e l}^{(\text {para })}=c \cdot \frac{E}{2(1+v)} \bar{\rho} \sin ^{2} \omega
\end{array}
$$

Care should be taken in the calculation of elastic-plastic core shear stiffness. This is because core plates in the loading direction of parallel-to-corrugations can yield by axial compression. In other words, the plastic core shear stiffness should be obtained from the ratio of shear stress and shear strain increments in the presence of an axial pre-stress which is greater than the yield stress. This loading situation corresponds to the "neutral loading condition on the yield surface" according to the theory of plasticity [99]. Modifying Becque's derivation for the relation between shear stress increment and shear strain increment in presence of axial load [69], the elastic-plastic shear stiffness of core plates for parallel-to-corrugations can be analytically attained by

$$
\begin{gathered}
G_{C, p l}^{(\text {para })}=\frac{E_{T} E}{(1+\kappa+2 \nu) E_{T}+(1-\kappa) E} \bar{\rho} \sin ^{2} \omega \\
S_{p l}^{(\text {Para })}=A_{e f f} \cdot G_{C, p l}^{(\text {para })}=c \cdot \frac{E_{T} E}{(1+\kappa+2 v) E_{T}+(1-\kappa) E} \bar{\rho} \sin ^{2} \omega
\end{gathered}
$$

where the parameter $\kappa$ is defined as the ratio of the plastic strain increment in the principal 2 direction (y-direction of the plate) to the plastic strain increment in the principal 1 direction (x-direction) under uniaxial compression in the $\mathrm{x}$-direction. (If the von Mises yield criterion is adopted, $\kappa=-\frac{1}{2}$ ) [69]. 
The inertia term, independent of material yielding and loading orientation, is given by

$$
(\rho A)^{(S W)}=(2 h+\bar{\rho} c) \rho
$$

\subsection{AXIAL STRESS WAVE PROPAGATION}

In this section, estimation of axial force distribution, which might be non-uniform in a column, is discussed. The force distribution, $\mathrm{P}(\mathrm{x}, \mathrm{t})$, is a coupling term between the axial and transverse equations of motion for the problems of monolithic solid columns and sandwich columns: See Eqns. (2.3) and (2.4) as well as Eqns. (2.7) and (2.8).

For convenience, consider the monolithic solid column problem first. The distribution in the sandwich columns can be obtained in a similar way.

Axial force distribution $\mathrm{P}(\mathrm{x}, \mathrm{t})$ is calculated from the change of axial deformation, $u^{(m)}$ and the transverse deflection, $\mathrm{w}^{(\mathrm{m})}(\mathrm{x}, \mathrm{t})$, as seen in Eqn. (2.22).

$$
P(x, t)=(A E)^{*} \frac{\partial u^{(m)}}{\partial x}-\frac{1}{2}(A E)^{*}\left[\left(\frac{\partial\left(w^{(m)}+w_{0}^{(m)}\right)}{\partial x}\right)^{2}-\left(\frac{\partial w_{0}^{(m)}}{\partial x}\right)^{2}\right]
$$

After substituting Eqn. (2.22) into Eqn. (2.3), $u^{(m)}$ is given by the sum of the solutions, $u_{1}^{(m)}$ and $u_{2}^{(m)}$ from the following two sub-problems [39].

$$
u^{(m)}=u_{1}^{(m)}+u_{2}^{(m)}
$$

1) Axial sub-problem (Problem I): Homogeneous partial differential equation (PDE) with nonhomogeneous boundary conditions.

2) Axial sub-problem (Problem II): Nonhomogeneous partial differential equation (PDE) with homogeneous boundary conditions (BCs). 
$\underline{\text { Axial sub-problem (Problem I) for axial stress distribution }}$

$$
\begin{gathered}
\frac{\partial^{2} u_{1}^{(m)}}{\partial x^{2}}-\frac{1}{\left(c_{e l}^{(m)}\right)^{2}} \frac{\partial^{2} u_{1}^{(m)}}{\partial t^{2}}=0 \\
\mathrm{BC}: \quad u_{1}^{(m)}(0, t)=V t, \quad u_{1}^{(m)}(L, t)=0 \\
\mathrm{IC}: \quad u_{1}^{(m)}(x, 0)=0, \quad \frac{\partial u_{1}^{(m)}}{\partial t}(L, t)=0
\end{gathered}
$$

$\underline{\text { Axial sub-problem (Problem II) for axial stress distribution }}$

$$
\begin{gathered}
\frac{\partial^{2} u_{2}^{(m)}}{\partial x^{2}}-\frac{1}{\left(c_{e l}^{(m)}\right)^{2}} \frac{\partial^{2} u_{2}^{(m)}}{\partial t^{2}}=\frac{1}{2} \frac{\partial}{\partial x}\left[\left(\frac{\partial\left(w^{(m)}+w_{0}^{(m)}\right)}{\partial x}\right)^{2}-\left(\frac{\partial w_{0}^{(m)}}{\partial x}\right)^{2}\right] \\
\mathrm{BC}: \quad u_{2}^{(m)}(0, t)=0, \quad u_{2}^{(m)}(L, t)=0 \\
\mathrm{IC}: \quad u_{2}^{(m)}(x, 0)=0, \quad \frac{\partial u_{2}^{(m)}}{\partial t}(L, t)=0
\end{gathered}
$$

The two sub-problems have the following physical meaning: Problems I and II are related to the influences of axial inertia due to the end compression and to a change of transverse deflection, respectively. If the column deformation is entirely elastic,

$$
c_{e l}^{(m)}=\sqrt{E^{(m)} / \rho}
$$

For cylindrical bending ${ }^{1}$ of a plate, the equations (2.28) is transformed into

$$
c_{e l}^{(m)}=\sqrt{E^{(m)} /\left(1-v^{2}\right) \rho}
$$

1 One-dimensional plate problem where a plate width is large enough that all derivatives with respect to the width direction, $z$, are zero such as in a plane strain problem $[100,101]$. In the sandwich plate buckling problem, it is possible to assume this condition if plate width is greater than core height. [65] 
The homogeneous PDE (2.24) is a well-known one-dimensional wave equation. Its elastic solution, $u_{1}^{(m)}$, is given in Eqn. (2.30), which can be also represented by a combination of step functions.

$$
u_{1}^{(m)}=\frac{V x t}{L}+\frac{2 V L}{c_{e l}^{(m)} \pi^{2}} \sum_{n=1}^{\infty} \frac{(-1)^{n}}{n^{2}} \sin \left(\frac{n \pi x}{L}\right) \sin \left(\frac{c_{e l}^{(m)} n \pi t}{L}\right)
$$

Thus, the axial force $\mathrm{P}_{1}(\mathrm{x}, \mathrm{t})$ associated with $u_{1}^{(m)}$ in Problem I is built up by the propagation and reflection of axial stress waves, which will be detailed in Section 2.3.1. On the other hand, the elastic solution, $u_{2}^{(m)}$, of Problem II is a correction term considering the axial inertia from the change of transverse deflection, $w^{(m)}(x, t)$.

$$
\begin{aligned}
& u_{2}^{(m)}(x, t) \sim \sum a_{n}(t) \sin \frac{n \pi x}{L} \\
& =\sum_{n=1}^{\infty}\left\{\frac{c_{e l}^{(m)}}{L} \int_{0}^{t}\left[\int_{0}^{L}\left[\left(\frac{\partial\left(w+w_{o}\right)}{\partial x}\right)^{2}-\left(\frac{\partial w_{o}}{\partial x}\right)^{2}\right] \cdot \cos \frac{n \pi x}{L} d x\right] \cdot \sin \frac{c_{e l}^{(m)} n \pi(t-\tau)}{L} d \tau\right\} \cdot \sin \frac{n \pi x}{L}
\end{aligned}
$$

The derivations of elastic solutions in Eqns. (2.30) and (2.31) are given in Appendix A.

According to Sevin [39], the axial inertia effect due to the change of transverse deflection, $w^{(m)}(x, t)$, was observed not to be significant until the axial force reaches the maximum peak load. And thus, the axial inertia effect associated with $w^{(m)}(x, t)$ can be ignored for the calculation of $\mathrm{P}_{2}(\mathrm{x}, \mathrm{t})$. Accordingly, Eqn. (2.26) with Eqn. (2.22) is reduced to

$$
\frac{\partial P_{2}}{\partial x}=0
$$

leading $\mathrm{P}_{2}$ to a function of time only. 


$$
P_{2}(t)=\frac{(A E)^{*}}{2} \int_{0}^{L}\left[\left(\frac{\partial\left(w+w_{o}\right)}{\partial x}\right)^{2}-\left(\frac{\partial w_{o}}{\partial x}\right)^{2}\right] d x
$$

By ignoring the axial inertia in Problem II, a great deal of computation effort can be saved compared with the application of a complex equation such as Eqn. (2.31).

In summary, the estimation of axial force distribution is given by summing the axial forces, $\mathrm{P}_{1}(\mathrm{x}, \mathrm{t})$ and $\mathrm{P}_{2}(\mathrm{t})$, resulting from an end compression and a change of transverse deflection, respectively. In particular, $\mathrm{P}_{1}(\mathrm{x}, \mathrm{t})$ can be estimated from the theory of elastic and elastic-plastic wave propagation which will be discussed in Section 2.3.1, and thus the elastic and elastic-plastic stress distribution from the theory will be adopted for the semi-analytical models of monolithic columns and sandwich columns.

\subsubsection{Theoretical Background for Stress Wave Propagation}

In this subsection, the stress wave propagation in the compression of monolithic columns will be explained graphically. The estimation method for axial force distribution, $\mathrm{P}_{1}(\mathrm{x}, \mathrm{t})$, will be employed for the analytical model rather than using a complex equation such as Eqn.(2.30) and Eqn. (2.31).

Fig. 2.4(a)(b)(c) show the stress wave propagation in an elastic monolithic solid column compressed at one end at a constant compression velocity: (a) the stress distribution of the column with respect to time $t=t_{1 e}, t_{2 e}, t_{3 e}$, (b) the corresponding Lagrange $x$-t diagram, and (c) the material states are shown. When the column is compressed, an incident elastic stress wave of $\Delta \sigma_{e l}=\Delta \sigma_{e l}^{(m)}=\rho c_{e l}^{(m)} V$ travels toward the other end at a wave speed of $c_{e l}=c_{e l}^{(m)}$ until it is reflected at the other end as shown in the diagram at $\mathrm{t}=\mathrm{t}_{1 \mathrm{e}}$ of Fig. 2.4(a). After reflection, the magnitude of the propagating 


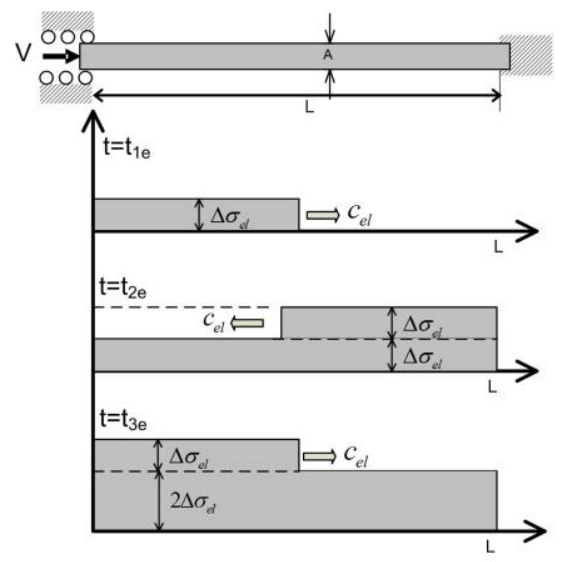

(a)
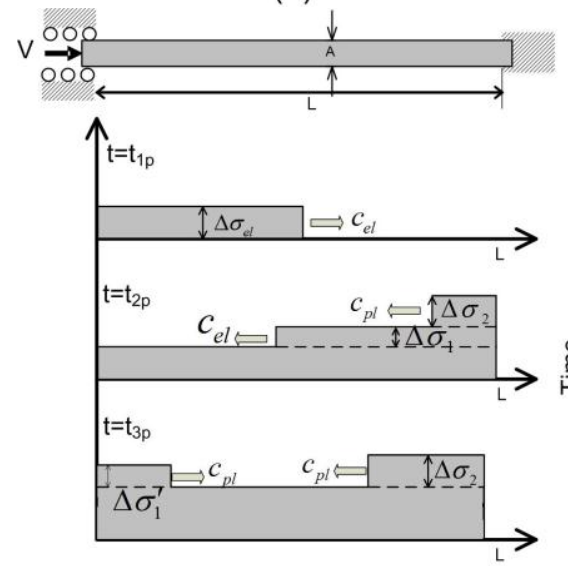

(d)

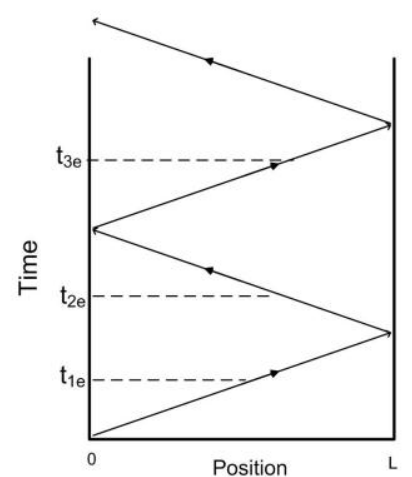

(b)

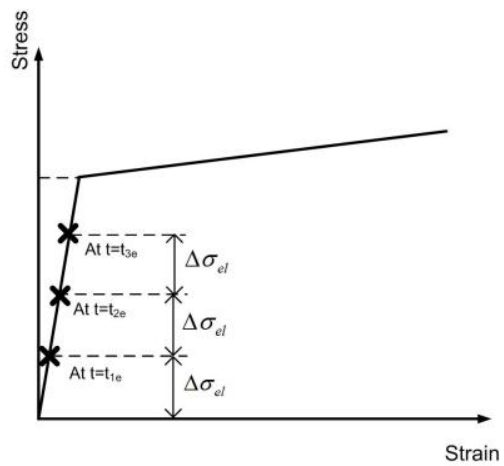

(c)

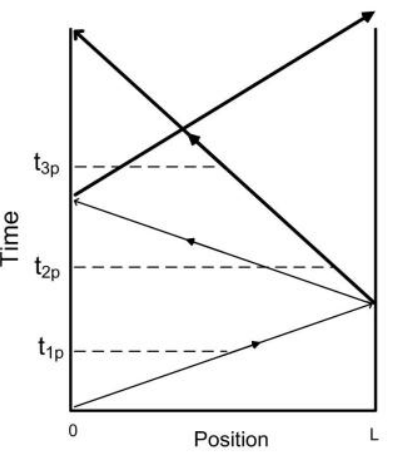

(e)

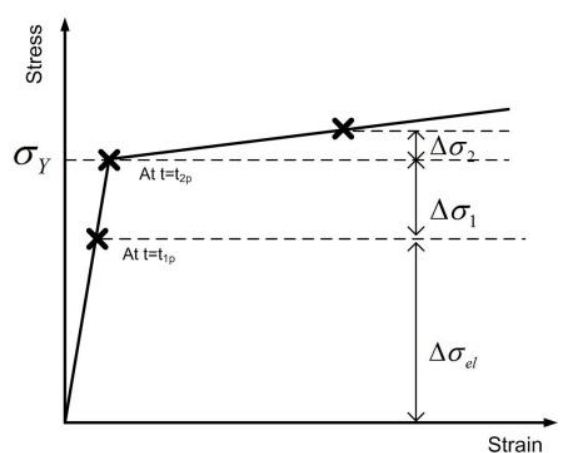

(f)

Figure 2.4: Stress distribution diagram (monolithic solid columns), Lagrange $x-t$ diagram and the corresponding material state for (a)(b)(c) an elastic stress wave propagation, and (d)(e)(f) an elastic-plastic stress wave propagation.

axial stress is increased by $\Delta \sigma_{e l}=\Delta \sigma_{e l}^{(m)}$ and its direction is reversed as illustrated in the stress distribution diagram at $\mathrm{t}=\mathrm{t}_{2 \mathrm{e}}$ of Fig. 2.4(a). Wave reflections leading to increase in stress magnitude are repeated in the same manner as long as the column material remains elastic. A Lagrange $\mathrm{x}$-t diagram indicating the location of the elastic wave front with respect to time is described in Fig. 2.4(b), and the material states in the stress-strain curve after each stress reflection are also indicated in Fig. 2.4(c).

If stress exceeds the yield limit, either because of numerous wave reflections or a high-applied velocity, a plastic wave in addition to the elastic wave is generated and 
propagates independently as shown in Fig. 2.4(d)(e)(f) [70-73]. Because of the condition $\frac{V}{c_{e l} \epsilon_{Y}}<1$ specified in this chapter, the elastic-plastic stress propagation occurs only by repeated wave reflections. At time $\mathrm{t}=\mathrm{t}_{\mathrm{lp}}$ in Fig. 2.4(d), an elastic wave of $\Delta \sigma_{e l}=$ $\Delta \sigma_{e l}^{(m)}=\rho c_{e l}^{(m)} V$ propagates toward the right end. If the deformation remains elastic, the amount of stress should be increased by $\Delta \sigma_{\mathrm{el}}$ when it reaches the end. However, if the increment in stress causes the stress to be greater than the elastic limit, two wave fronts of $\Delta \sigma_{1}=\sigma_{Y}^{(m)}-\sigma_{1}$ and $\Delta \sigma_{2}=\sqrt{\frac{E_{T}^{\prime}}{E /\left(1-v^{2}\right)}}\left(\Delta \sigma_{e l}-\Delta \sigma_{1}\right)$ propagate independently with the respective wave speeds of $c_{e l}=c_{e l}^{(m)}$ and $c_{p l}=c_{p l}^{(m)}$ as shown at the time $\mathrm{t}=\mathrm{t}_{2 \mathrm{p}}$ in Fig. 2.4(d). Here, let $\sigma_{1}$ denote the axial stress just before the elastic-plastic wave reflection, and

$$
c_{P l}^{(m)}=\sqrt{E_{T}^{\prime(m)} / \rho}
$$

in which $E_{T}^{\prime}$ is the tangent modulus of the plane strain true stress versus logarithmic strain curve of the parent material. For convenience, $E_{T}^{\prime}$ of a linear strain-hardening material can be approximated as a function of plastic hardening modulus, $E_{P}=d \sigma_{e q} / d p$, and elastic material properties, $\mathrm{E}$ and $v$.

$$
E_{T}^{\prime(m)}=\frac{1}{\left(1 / E-v / 2 E+3 / 4 E_{p}\right)}
$$

As a result, the faster wave of $\Delta \sigma_{1}$ with $\mathrm{c}_{\mathrm{el}}$ arrives earlier at the other end. After the reflection of the wave, it changes its direction and travels with a plastic wave speed of $\mathrm{c}_{\mathrm{pl}}$ and an increased amount of stress from the wave reflection, $\left.\Delta \sigma_{1}^{\prime}\right|_{t 2<t<t 3}=\sqrt{\frac{E_{T}^{\prime}}{E /\left(1-v^{2}\right)}}$. 
$\Delta \sigma_{1}$ as shown in the diagram of Fig. 2.4(d) at the time between $t_{2 p}$ and $t_{3 p}$. Fig. 2.4(e) shows the corresponding Lagrange $\mathrm{x}$-t diagram in which the thin and thick lines represent elastic and plastic wave fronts, respectively.

In short, the axial force distribution with respect to time, $P_{1}(x, t)$, can be obtained from such distribution as either Figs. 2.4(a) or (d).

\subsubsection{Analogy between Monolithic Solid Columns and Sandwich Columns}

The axial force distribution $\mathrm{P}_{1}(\mathrm{x}, \mathrm{t})$ of sandwich columns can be approximated by an analogy between a monolithic solid column and a sandwich column. Specifically, elastic and elastic-plastic wave propagation properties in sandwich columns can be derived from the in-plane stiffness and inertia, and it is assumed that the wave propagation is onedimensional neglecting any variations in the $\mathrm{y}$ and $\mathrm{z}$ directions [70-73]. Compare Figures 2.4 and 2.5 .

For a corrugated core sandwich column, laminate composite theory and stress wave propagation theory are used to describe the state of the stress wave. For loading perpendicular-to-corrugation, the apparent wave speeds ${ }^{2}$ of sandwich columns, $c_{e l}^{\text {(perp) }}$ and $c_{p l}^{(p e r p)}$, in the elastic and plastic region can be obtained by neglecting the contribution of a corrugated core to the in-plane stiffness. The expressions are based on the treatment of a corrugated core as a continuous core of negligible in-plane core stiffness.

2 The word "apparent" is added after confirming FE simulations on the axial wave characteristics of sandwich columns. In a microscopic time scale during which an axial wave propagates one unit cell or so, the axial wave front in sandwich columns does not look planar in the y-z plane due to the effect of the core (rather, zig-zag in top and bottom faces). On the other hand, the reaction force increase at one end is achieved in stepwise pattern macroscopically. 


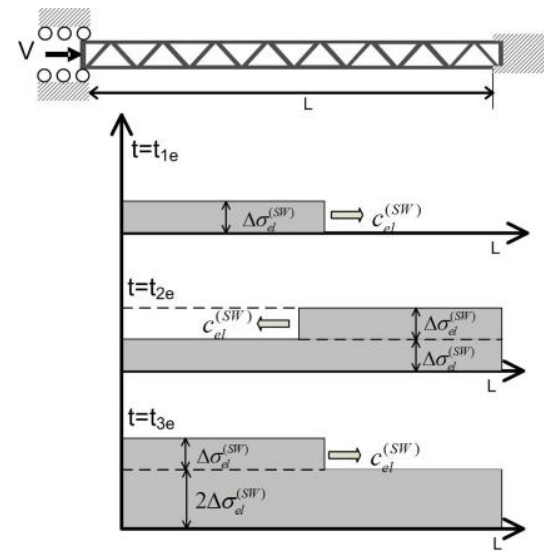

(a)

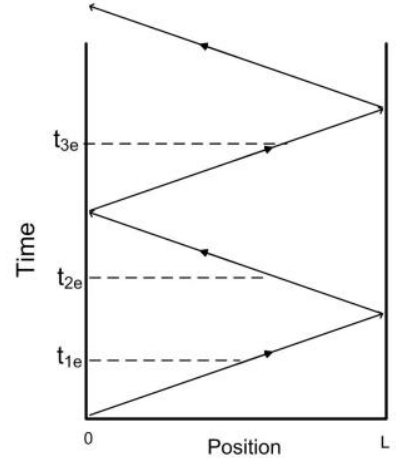

(b)

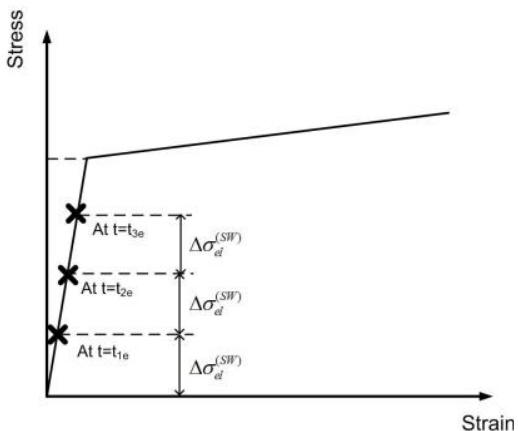

(c)

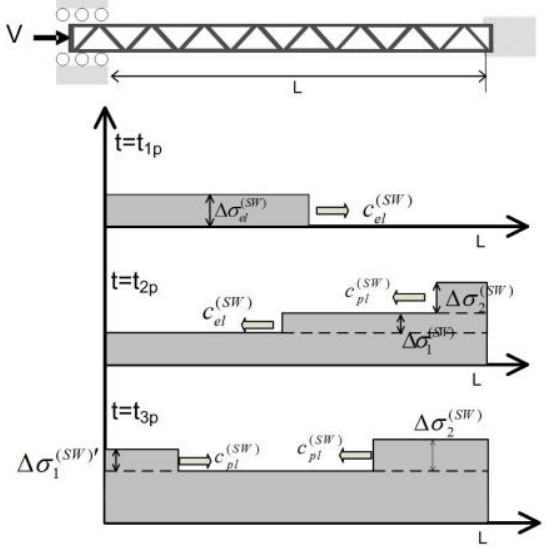

(d)

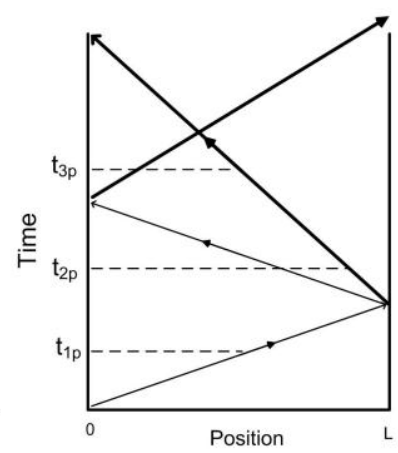

(e)

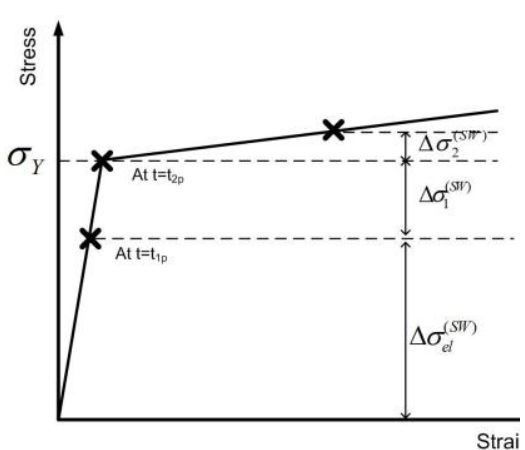

(f)

Figure 2.5: Stress distribution diagram (sandwich columns), Lagrange $\mathrm{x}-\mathrm{t}$ diagram and the corresponding material state for $(a)(b)(c)$ an elastic stress wave propagation, and $(d)(e)(f)$ an elastic-plastic stress wave propagation.

$$
\begin{gathered}
c_{e l}^{(\text {perp })}=\sqrt{\frac{E}{\left(1-v^{2}\right)} \frac{2 h}{(2 h+\bar{\rho} c) \rho}} \\
\Delta \sigma_{e l}^{(\text {perp })}=\sqrt{\frac{2 h E(2 h+\bar{\rho} c) \rho}{1-v^{2}}} V \\
c_{p l}^{(\text {perp })}=\sqrt{2 h E_{T}^{\prime} /(2 h+\bar{\rho} c) \rho} \\
\sigma_{Y}^{(\text {perp })}=\frac{\sigma_{Y}}{\sqrt{1-v_{P}+v_{P}^{2}}} \\
P_{Y}^{(\text {perp })}=\frac{2 h \cdot \sigma_{Y}}{\sqrt{1-v_{P}+v_{P}^{2}}}
\end{gathered}
$$


On the other hand, the elastic and elastic-plastic wave speeds where loading is parallel-to-corrugations loading are approximately the same as that of monolithic solid columns because the core plates carry a part of axial loads together with face sheets.

$$
\begin{aligned}
& c_{e l}^{(\text {para })}=\sqrt{\frac{2 h E /\left(1-v^{2}\right)+\bar{\rho} c E}{(2 h+\bar{\rho} c) \rho}} \\
& \Delta \sigma_{e l}^{(\text {para })}=\sqrt{\left[\frac{2 h E /\left(1-v^{2}\right)+\bar{\rho} c E}{(2 h+\bar{\rho} c)}\right] \rho \cdot V} \\
& c_{p l}^{(\text {para })}=\sqrt{\frac{2 h E_{T}^{\prime}+\bar{\rho} c E_{T}}{(2 h+\bar{\rho} c) \rho}} \\
& P_{Y}^{(\text {para })}=\frac{2 h \cdot \sigma_{Y}}{\sqrt{1-v_{P}+v_{P}^{2}}}+\bar{\rho} c \sigma_{Y}
\end{aligned}
$$

\subsection{SEMI-ANALYTICAL MODELS}

\subsubsection{Semi-analytical Model for Monolithic Solid Columns}

As shown in the previous section, the axial force distribution, $\mathrm{P}(\mathrm{x}, \mathrm{t})$ is the sum of the axial forces due to the strains of end compression $\left(\mathrm{P}_{1}(\mathrm{x}, \mathrm{t})\right)$ and transverse deflection $\left(\mathrm{P}_{2}(\mathrm{t})\right)$. By using the theory of elastic-plastic wave propagation, axial force due to the end compression, $\mathrm{P}_{1}(\mathrm{x}, \mathrm{t})$, can be obtained. Neglecting the influence of axial inertia due to the change of transverse displacement, we defined the axial force as a function of time only, $\mathrm{P}_{2}(\mathrm{t})$. Accordingly, the axial force, $\mathrm{P}(\mathrm{x}, \mathrm{t})=\mathrm{P}_{1}(\mathrm{x}, \mathrm{t})+\mathrm{P}_{2}(\mathrm{t})$, can be expressed in a different form: 


$$
P(x, t)=P_{0}+\Delta P_{\text {short }}(x, t)+\Delta P_{\text {long }}(t)
$$

where $\Delta P_{\text {short }}$ is the increased amount of axial force resulting from one wave reflection, $\Delta P_{\text {long }}$ is the axial force caused from transverse deflection, $\mathrm{P}_{0}$ is a reference value during the period for a wave to travel along a column length, and updated every wave reflection.

The governing equation for a monolithic solid column is solved using the Galerkin method [100].

$$
\begin{gathered}
\int_{0}^{L}\left[\frac{\partial^{2}}{\partial x^{2}}\left(D^{(m)} \frac{\partial^{2} w^{(m)}}{\partial x^{2}}\right)+\frac{\partial}{\partial x}\left(P(x, t) \frac{\partial\left(w^{(m)}+w_{0}^{(m)}\right)}{\partial x}\right)+\rho A \frac{\partial^{2} w^{(m)}}{\partial t^{2}}\right] \cdot \delta w^{(m)} d x=0 \\
w^{(m)}(x, t)=\sum_{i=1}^{n} T_{i}(t) \cdot W_{i}(x) \\
w_{0}^{(m)}(x)=\sum_{i=1}^{n} \xi_{i} \cdot W_{i}(x) \\
\delta w^{(m)}=\sum_{i=1}^{n} \delta T_{i} \cdot W_{i}(x)
\end{gathered}
$$

The transverse motion, $\mathrm{w}^{(m)}(x, t)$, initial curvature imperfection, $w_{0}^{(m)}(x)$, and virtual displacement, $\delta \mathrm{w}^{(m)}$, are given in the form of an eigenfunction expansion, up to $\mathrm{W}_{18}(\mathrm{x})$, which are obtained from the static buckling analysis. Although Eqn. (2.45) includes the spatial discontinuity of $\mathrm{P}(\mathrm{x}, \mathrm{t})$ and $\mathrm{D}^{(\mathrm{m})}(\mathrm{x}, \mathrm{t})$, it is assumed that the continuity and differentiability of $\mathrm{w}^{(m)}(x, t)$ is preserved during the dynamic global buckling motion.

The base functions, $\mathrm{W}_{\mathrm{n}}(\mathrm{x})$, for $w^{(m)}(x, t), w_{0}^{(m)}(x), \delta w^{(m)}$ are divided into symmetric and anti-symmetric modes depending on their mode shapes: the symmetric modes are the (2k-1)-th modes whereas the (2k)-th modes are anti-symmetric modes. Constants up to ten decimal points are used in the implementation of the semi-analytical 
model, and are listed in Table 2.1.

$$
\text { Symmetric modes (odd n): } \quad W_{n}(x)=\frac{1}{2}\left(1-\cos \frac{(n+1) \pi x}{L}\right)
$$

Antisymmetric modes (even n): $W_{n}(x)=A_{n}\left(\sin k_{n} x-k_{n} x-\frac{k_{n} L}{2} \cos k_{n} x+\frac{k_{n} L}{2}\right)$

Substituting Eqns. (2.46) through (2.48) into the governing equation (2.45), the integral form of the governing equation is arranged into a system of ODEs with respect to $\mathrm{T}_{\mathrm{n}}(\mathrm{t})$. Finally, a $4^{\text {th }}$ order Runge-Kutta method was employed using FORTRAN code for solving the system of ODEs (2.50).

$$
[\rho A]_{i j}^{(m)}\{\ddot{T}\}_{j}^{(m)}+\left([D]_{i j}^{(m)}+[P]_{i j}^{(m)}\right)\{T\}_{j}^{(m)}=\left[{ }^{0} P\right]_{i}^{(m)}
$$

where $\{\ddot{T}\}_{j}^{(m)}=\left(\begin{array}{c}\ddot{T}_{1} \\ \ddot{T}_{2} \\ \vdots \\ \ddot{T}_{n}\end{array}\right),\{T\}_{j}^{(m)}=\left(\begin{array}{c}T_{1} \\ T_{2} \\ \vdots \\ T_{n}\end{array}\right) ; \quad[\rho A]_{i j}^{(m)},[D]_{i j}^{(m)}$ and $[P]_{i j}^{(m)}$ are matrices whose (ij)th components are $\int_{0}^{L} \rho A W_{i} W_{j} d x, \int_{0}^{L} \frac{d^{2}}{d x^{2}}\left(D(x) \cdot \frac{d^{2} W_{j}}{d x^{2}}\right) W_{i} d x$, and $\int_{0}^{L} \frac{d}{d x}\left(P(x, t) \frac{d W_{j}}{d x}\right) \cdot W_{i} d x$, respectively; $\left[{ }^{0} P\right]_{i}^{(m)}$ is a vector whose $\mathrm{i}$-th component is $-\int_{0}^{L} \frac{d}{d x}\left(\sum_{k=1}^{n} \xi_{k} P(x, t) \frac{d W_{k}}{d x}\right) \cdot W_{i} d x$.

Equation (2.50) is solved until a failure criterion is satisfied. The failure is defined as a load-drop of reaction forces, which is simultaneous with the time of the maximum

Table 2.1. Constants for antisymmetric modes.

\begin{tabular}{cccccccccc}
\hline $\begin{array}{l}\text { Eigenmode } \\
\text { constant }\end{array}$ & $\mathrm{N}=2$ & $\mathrm{~N}=4$ & $\mathrm{~N}=6$ & $\mathrm{~N}=8$ & $\mathrm{~N}=10$ & $\mathrm{~N}=12$ & $\mathrm{~N}=14$ & $\mathrm{~N}=16$ & $\mathrm{~N}=18$ \\
\hline \hline $\mathrm{K}_{\mathrm{n}} \mathrm{L}$ & 8.987 & 15.451 & 21.808 & 28.1320 & 34.442 & 40.743 & 47.037 & 53.332 & 59.623 \\
$\mathrm{An}$ & 6.283 & 12.566 & 18.849 & 25.133 & 31.416 & 37.699 & 43.982 & 50.265 & 56.549 \\
\hline
\end{tabular}


reaction force. When this type of instability in a compressive member occurs, excessive deformation together with a high deformation rate leads to a sudden loss of load-carrying capacity. In this regard, a failure criterion is defined such that a significant load drop occurs if the extension rate of column mid-surface exceeds the compressive rate [74].

$$
\frac{d \Delta l_{e x t}}{d t} \geq \frac{d \Delta l_{c o m p}}{d t}
$$

where $\frac{d \Delta l_{\text {ext }}}{d t} \approx \int_{0}^{L}\left[\frac{d}{d t}\left(\frac{d w^{(m)}}{d x}\right)\right]\left(\frac{d w^{(m)}}{d x}\right) d x+\int_{0}^{L}\left[\frac{d}{d t}\left(\frac{d w^{(m)}}{d x}\right)\right]\left(\frac{d w_{0}^{(m)}}{d x}\right) d x$

$$
\frac{d \Delta l_{\text {comp }}}{d t} \approx V
$$

The model explained so far pertains to a rate-independent monolithic solid column. To include the material rate-dependence, especially for SS304, the semi-analytical model considers the rate-sensitive material as a rate-independent material with elevated yield strength and adjusted strain-hardening modulus [75].

$$
\begin{gathered}
\sigma_{Y}^{(m)}=\frac{R \cdot \sigma_{Y}}{\sqrt{1-v_{P}+v_{P}^{2}}} \\
E_{T}^{\prime(m)}=\frac{1}{1 /(E)-v /(2 E)+3 /\left(4 R E_{p}\right)}
\end{gathered}
$$

where $\mathrm{R}$ is the dynamic yield strength enhancement ratio, $\mathrm{R}=\frac{\sigma_{Y}(\dot{p})}{\sigma_{Y}^{0}}$, (i.e., the ratio of the dynamic yield strength and the quasi-static one) in terms of equivalent plastic strain rate, $\dot{p} \approx \frac{2}{\sqrt{3}} \frac{V}{L}$. The semi-analytical model for the problem of monolithic solid columns is summarized in Fig. 2.6. 


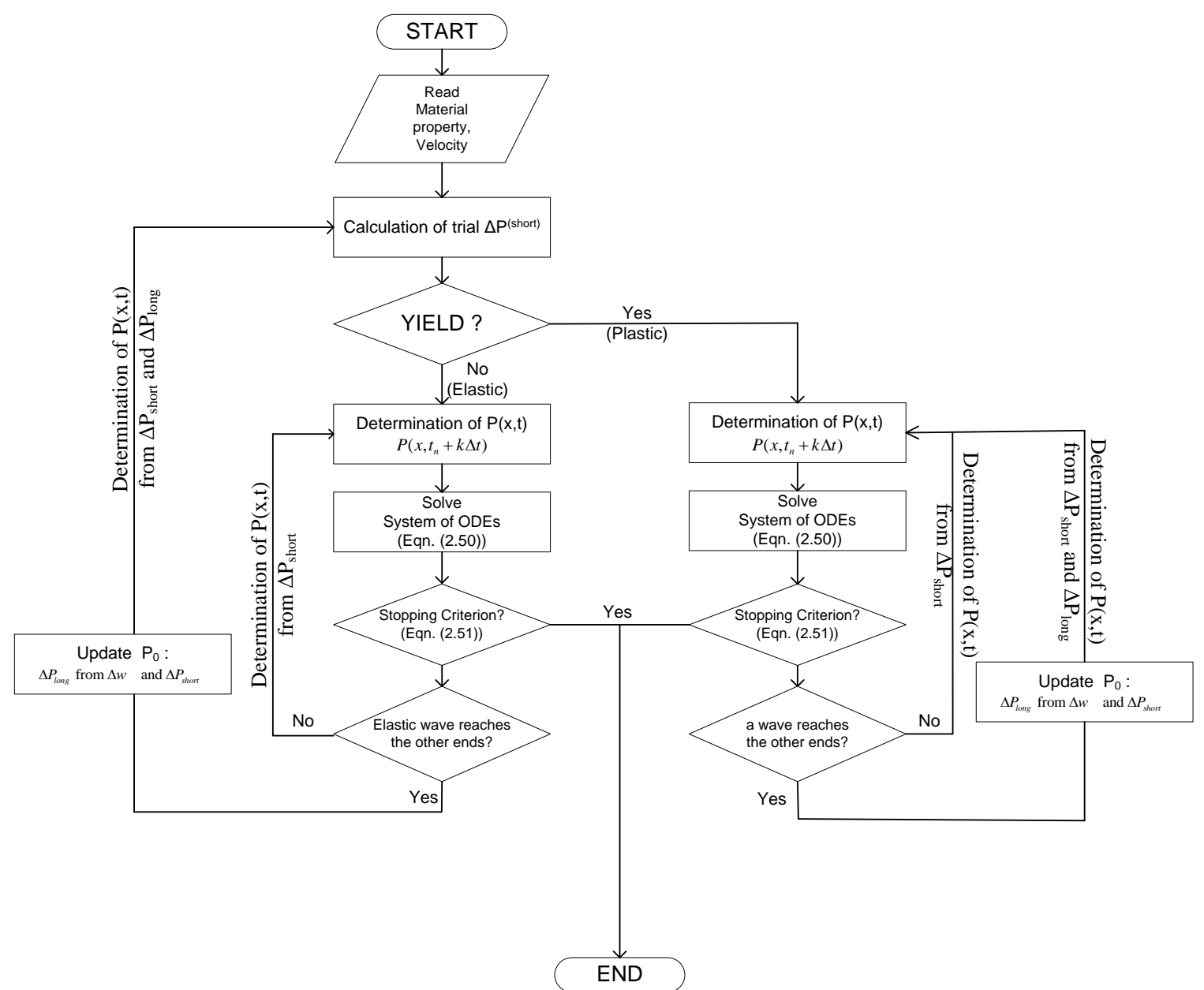

Figure 2.6: Flow chart of the semi-analytical model for monolithic solid columns.

\subsubsection{Semi-analytical Model for Corrugated Core Sandwich Columns}

Similar to the semi-analytic approach for the monolithic solid column problem, the governing equation of corrugated core sandwich columns, Eqn.(2.11), can be solved using the Galerkin method.

$$
\begin{gathered}
\int_{0}^{L}\left((\rho A)^{(S W)} \frac{\partial^{2} w^{(S W)}}{\partial t^{2}}\right) \delta w \cdot d x+\int_{0}^{L}\left(\frac{d}{d x}\left(P \cdot \frac{\partial w^{(S W)}}{\partial x}\right)\right) \delta w \cdot d x+\int_{0}^{L}\left(\frac{d^{2}}{d x^{2}}\left(D^{(S W)} \frac{\partial^{2} w^{(S W)}}{\partial x^{2}}\right)\right) \delta w \cdot d x \\
-\int_{0}^{L}\left(\frac{d^{2}}{d x^{2}}\left(\frac{D^{(S W)} \rho A^{(S W)}}{S^{(S W)}} \frac{\partial^{2} w}{\partial t^{2}}\right)\right) \delta w \cdot d x-\int_{0}^{L}\left(\frac{d^{2}}{d x^{2}}\left(\frac{D^{(S W)}}{S^{(S W)}} \cdot \frac{d}{d x}\left[P \frac{\partial w^{(S W)}}{\partial x}\right]\right)\right) \delta w \cdot d x \\
=-\int_{0}^{L}\left(\frac{d}{d x}\left(P^{(S W)} \cdot \frac{\partial w_{0}^{(S W)}}{\partial x}\right)\right) \delta w \cdot d x+\int_{0}^{L}\left(\frac{d^{2}}{d x^{2}}\left(\frac{D^{(S W)}}{S^{(S W)}} \cdot \frac{d}{d x}\left[P \frac{\partial w_{0}^{(S W)}}{\partial x}\right]\right)\right) \delta w \cdot d x
\end{gathered}
$$


Substituting Eqns. (2.55) through (2.57) into Eqn. (2.54), the integral form of the governing equation is transformed into a system of ordinary differential equations, Eqn. (2.58), including spatially discontinuous functions such as $\mathrm{P}(\mathrm{x}, \mathrm{t}), \mathrm{D}^{(\mathrm{SW})}$ and $\mathrm{S}^{(\mathrm{SW})}$. The system of ODEs is solved till the failure criterion, Eqn. (2.51) defined in Section 2.4.1, is satisfied.

$$
\begin{gathered}
w^{(S W)}(x, t)=\sum_{i=1}^{n} T_{i}(t) \cdot W_{i}(x) \\
w_{0}^{(S W)}(x)=\sum_{i=1}^{n} c_{i} \cdot W_{i}(x) \\
\delta w^{(S W)}=\sum_{i=1}^{n} \delta T_{i} \cdot W_{i}(x) \\
\left([\rho A]_{i j}^{(S W)}-\left[\frac{D \rho A}{S}\right]_{i j}^{(S W)}\right)\{\ddot{T}\}_{j}^{(S W)}+\left([D]^{(S W)}+[P]^{(S W)}+\left[\frac{D P}{S}\right]_{i j}^{(S W)}\right)\{T\}_{j}^{(S W)}=\left[{ }^{0} P\right]_{i}^{(S W)}+\left[\frac{D P}{S}\right]_{i}^{(S W)}
\end{gathered}
$$

where $\{\ddot{T}\}_{j}^{(S W)}=\left(\begin{array}{c}\ddot{T}_{1} \\ \ddot{T} \\ \vdots \\ \ddot{T}\end{array}\right),\{T\}_{j}^{(S W)}=\left(\begin{array}{c}T_{1} \\ T_{2} \\ \vdots \\ T_{n}\end{array}\right) ;[\rho A]_{i j}^{(S W)},[D]_{i j}^{(S W)},[P]_{i j}^{(S W)},\left[\frac{D \rho A}{S}\right]_{i j}^{(S W)},\left[\frac{D P}{S}\right]_{i j}^{(S W)}$ are matrices whose ij-th components are $\int_{0}^{L} \rho A W_{i} W_{j} d x, \int_{0}^{L} \frac{d^{2}}{d x^{2}}\left(D^{(S W)}(x, t) \cdot \frac{d^{2} W_{j}}{d x^{2}}\right) W_{i} d x$, $-\int_{0}^{L} \frac{d}{d x}\left(P(x, t) \frac{d W_{j}}{d x}\right) \cdot W_{i} d x, \int_{0}^{L} \frac{d^{2}}{d x^{2}}\left(\frac{D^{(S W)}(x, t) \rho A}{S^{(S W)}(x, t)} \cdot \frac{d^{2} W_{j}}{d x^{2}}\right) W_{i} d x$, and $\int_{0}^{L} \frac{d^{2}}{d x^{2}}\left(\frac{D^{(S W)}(x, t)}{S^{(S W)}(x, t)} \cdot \frac{d}{d x}\left(P(x, t) \frac{d W_{j}}{d x}\right)\right) W_{i} d x$, respectively; $\left[{ }^{0} P\right]_{i}^{(S W)}$ and $\left[\frac{{ }^{0} D P}{S}\right]_{i}^{(S W)}$ are vectors whose i-th components are $-\int_{0}^{L} \frac{d}{d x}\left(\sum_{k=1}^{n} \xi_{k} P(x, t) \frac{d W_{k}}{d x}\right) \cdot W_{i} d x$ and $\int_{0}^{L} \frac{d^{2}}{d x^{2}}\left(\frac{D^{(S W)}(x, t)}{S^{(S W)}(x, t)} \cdot \sum_{k=1}^{n} \xi_{k} P(x, t) \frac{d W_{k}}{d x}\right) \cdot W_{i} d x$ 
Similar to the way of including the material rate-dependence in the monolithic solid column semi-analytical model, Perrone's approximation $[33,75,101]$ is employed for consideration of the material rate-dependence of corrugated core sandwich columns.

\subsection{FINITE ELEMENT METHOD}

Finite Element simulations are performed to validate the semi-analytical models for the global buckling response of monolithic solid columns and corrugated core sandwich columns under dynamic compression. A commercial FE package, ABAQUS/Explicit, is employed to calculate reaction forces and deformation shapes. Four categories of FE sandwich column models are constructed depending on parent materials and in-plane loading orientations: 1) Al6061-T6 sandwich columns compressed perpendicular-tocorrugations, 2) Al6061-T6 sandwich columns compressed parallel-to-corrugations, 3) SS304 sandwich columns compressed perpendicular-to-corrugations, and 4) SS304 sandwich columns compressed parallel-to-corrugations. In addition to those, FE models for monolithic solid columns are also constructed. Figures 2.7 and 2.8 show the FE models of A16061-T6 sandwich columns with loading perpendicular-to-corrugation and parallel-to-corrugations, respectively. FE sandwich columns are based on the crosssections (unit-cells) as shown in Fig. 2.9. For example, FE sandwich models for parallelto-corrugations are created by extruding the cross sections in the direction normal to the cross sectional plane while the ones for perpendicular-to-corrugations are created by 
(a)

(a)

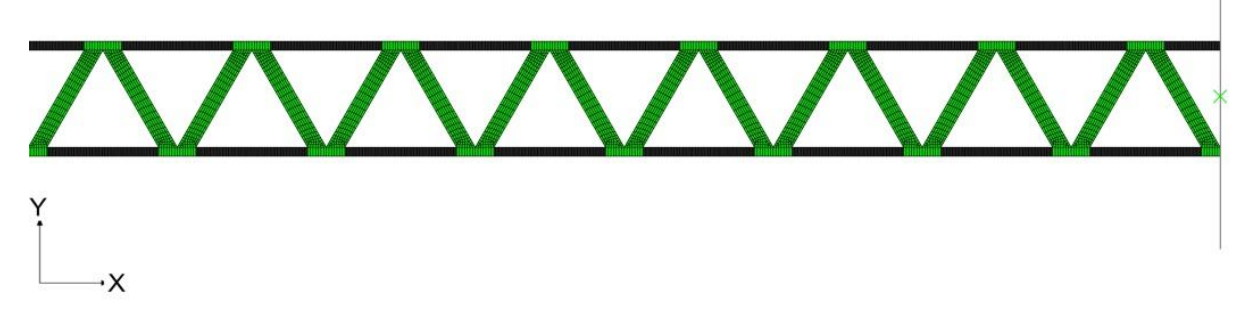

(b)

Figure 2.7: FE model of Al6061-T6 sandwich columns compressed perpendicular-to-corrugations; (a) a front view; (b) a side view.

(a)

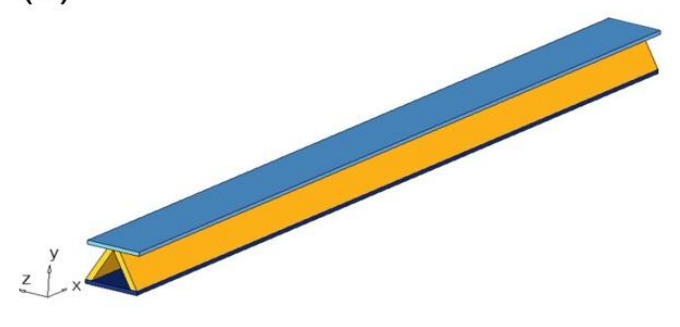

(b)

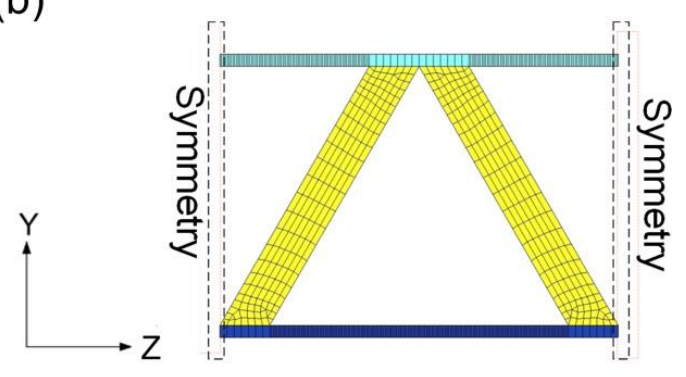

Figure 2.8: FE model of Al6061-T6 sandwich columns compressed parallel-to-corrugations: (a) an iso view; (b) a side view.

(a)

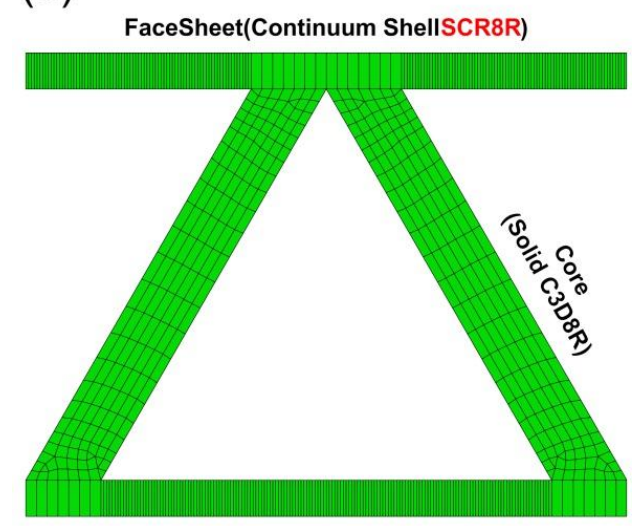

(b)

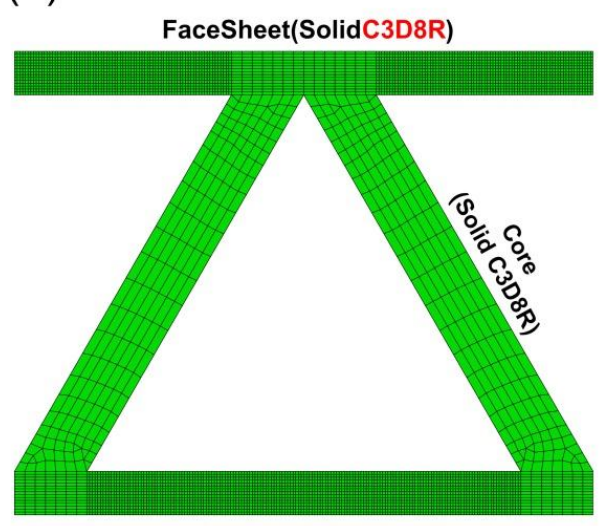

Figure 2.9: FE unitcell model for Al6061-T6 sandwich columns with face sheets meshed by (a) continuum shell elements; (b) Continuum solid elements.

duplicating the unit-cell to the desired length and extruding it by a unit length. Likewise, FE models of an SS304 column are constructed as shown in Fig. 2.10. 

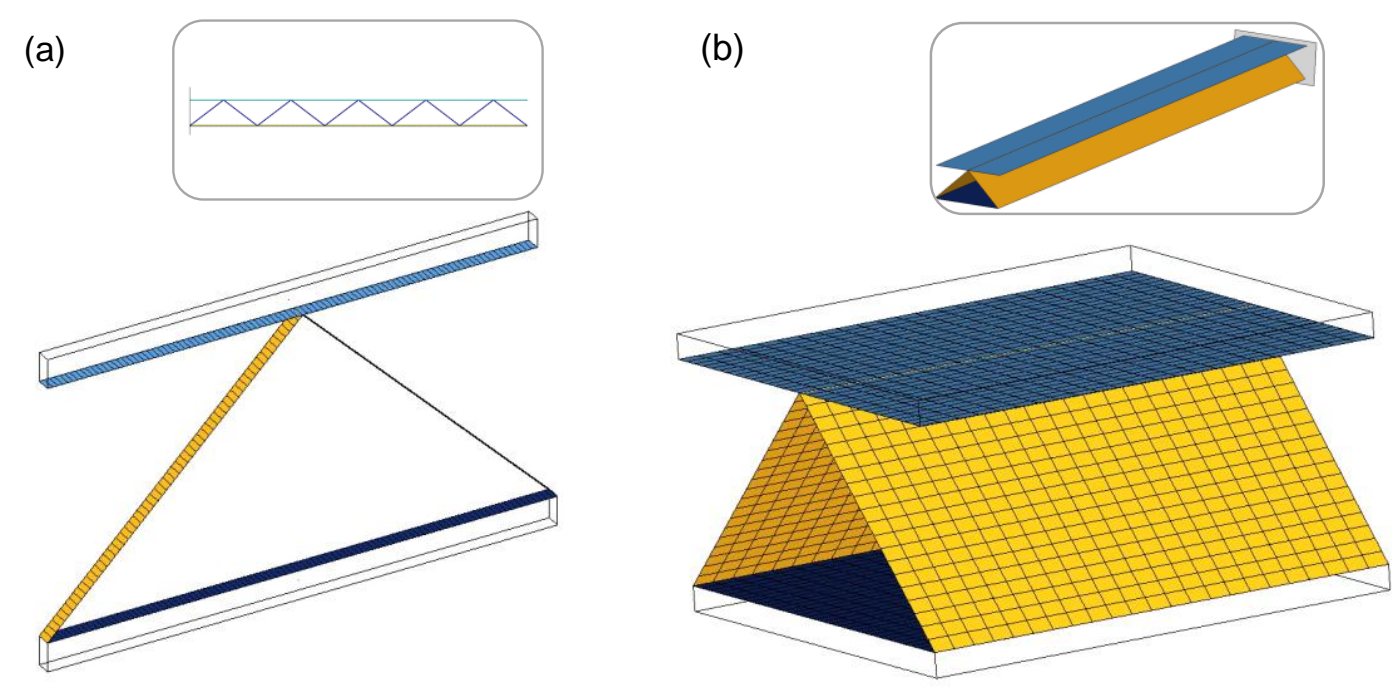

Figure 2.10: FE model of SS304 sandwich columns compressed (a) perpendicular-to-corrugations; (b) parallel-to-corrugations.

The FE model of A16061-T6 corrugated core sandwich columns employed solid continuum elements (C3D8R) and continuum shell elements (SC8R) for the modeling of a core and face section. If the ratio, $h /(2 l \cos w)$, between face sheet thickness and the span length of face sheet ligament is greater than 0.1 , continuum solid elements are used for the elements of face sheets.

On the other hand, SS304 corrugated core sandwich columns are meshed using conventional shell elements (S4R). It is noted that the reference planes for face sheets to be meshed are the closest surfaces to core parts whereas the mid-thickness planes are selected to be meshed for core parts as shown in Fig. 2.10. The solid lines describe physical face sheet entities while colored surfaces are the reference planes on which shell elements are meshed for face sheets. One reason for this selection is to be consistent with the DOF between elements of two parts, that is, there is no need to use connecting elements. 
The lowest static buckling modes, used as geometric curvature imperfections in the dynamic analyses, are obtained by performing additional FE static buckling analysis. The mesh for the static buckling analysis should be the same as that used for the dynamic simulation. The lowest static buckling mode recorded in the by-product (.fil file) of the eigen buckling analysis is inserted into the dynamic simulation. Consequently, the nodal positions, which define perfect column geometry initially in the pre-processing step, are relocated for the description of the imperfect column geometry and transported into the dynamic simulation. In this chapter, the global curvature imperfections are assigned in a form of the fundamental static buckling mode, $w_{0}(x)=\frac{\xi}{2}\left(1-\cos \frac{2 \pi x}{L}\right)$ with magnitudes $\xi=0.01 \mathrm{c}, 0.05 \mathrm{c}$ (occasionally, $\xi=0.1 \mathrm{c}$ ).

The parent material properties of Al6061-T6 and SS304 are characterized for the FE simulations and the semi-analytical model. The aluminum alloy was modeled as a rate-independent elastic-plastic material $[76,77]$ with linear strain-hardening employing the von Mises yield criterion while the material response of SS304 is described by a ratedependent [78] elastic-plastic model with the von Mises yield criterion and a bilinear strain-hardening.

The quasi-static material parameters for A16061-T6 and SS304 are determined from quasi-static experiments carried out by Biagi [28]. The quasi-static stress-strain curves of the two parent materials are shown in Figs. 2.11(a) and (b), respectively. A16061-T6 is adequately represented by Young's modulus E=75150(MPa), the yield stress, $\sigma_{\mathrm{Y}}=293.9(\mathrm{MPa})$ and the plastic hardening modulus, $E_{P}=\frac{d \sigma_{e q}}{d p}=534.2(\mathrm{MPa})$. And, Young's modulus and the two hardening moduli for the two plastic regions of SS304 are $\mathrm{E}=230769.2(\mathrm{MPa}), E_{P}^{(1)}=\frac{d \sigma_{e q}}{d p}=8117.65(M P a)$ and $E_{P}^{(2)}=\frac{d \sigma_{e q}}{d p}=2460.66(M P a)$, 


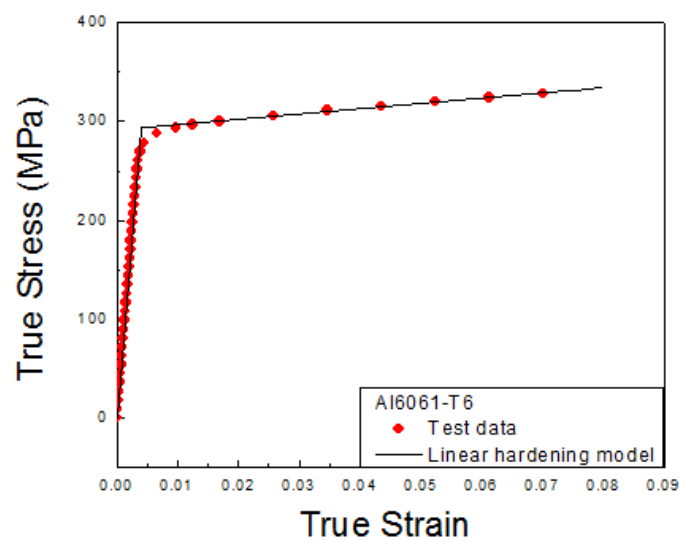

(a)

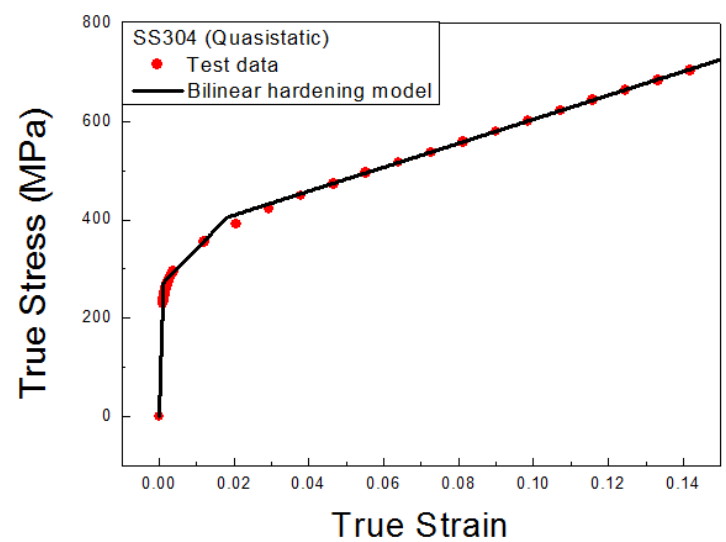

(b)

Figure. 2.11: Quasi-static stress-strain material behavior compared with the constitutive models of (a) AL6061-T6 and (b) SS304.

respectively. The material models are superimposed with the test data in Figs. 2.11(a) and (b). A kinematic hardening model for Al6061-T6, and an isotropic hardening model for SS304 are used to describe the strain-hardening regions in the FE simulations. The two hardening models are not significantly different unless the Bauschinger effect due to loading reversal is substantial.

The rate dependence of SS304 is taken into account by employing the CowperSymonds model [79], in which the dynamic yield strength enhancement ratio, $\mathrm{R}=\frac{\sigma_{Y}(\dot{p})}{\sigma_{Y}^{0}}$, (i.e. the ratio of the dynamic yield strength and the quasi-static one) is given by a function of equivalent plastic strain rate, $\dot{p}$. The following material parameters $\mathrm{D}=4920$ and $\mathrm{m}=0.154$ are used for the rate dependence of the stainless steel alloy $[3,51]$.

$$
R=\frac{\sigma_{y}(\dot{p})}{\sigma_{y}^{0}}=1+\left(\frac{\dot{p}}{D}\right)^{m}
$$

The densities of Al6061-T6 and SS304 are used as $2700 \mathrm{~kg} / \mathrm{m}^{3}$ and $7920 \mathrm{~kg} / \mathrm{m}^{3}$, respectively. 

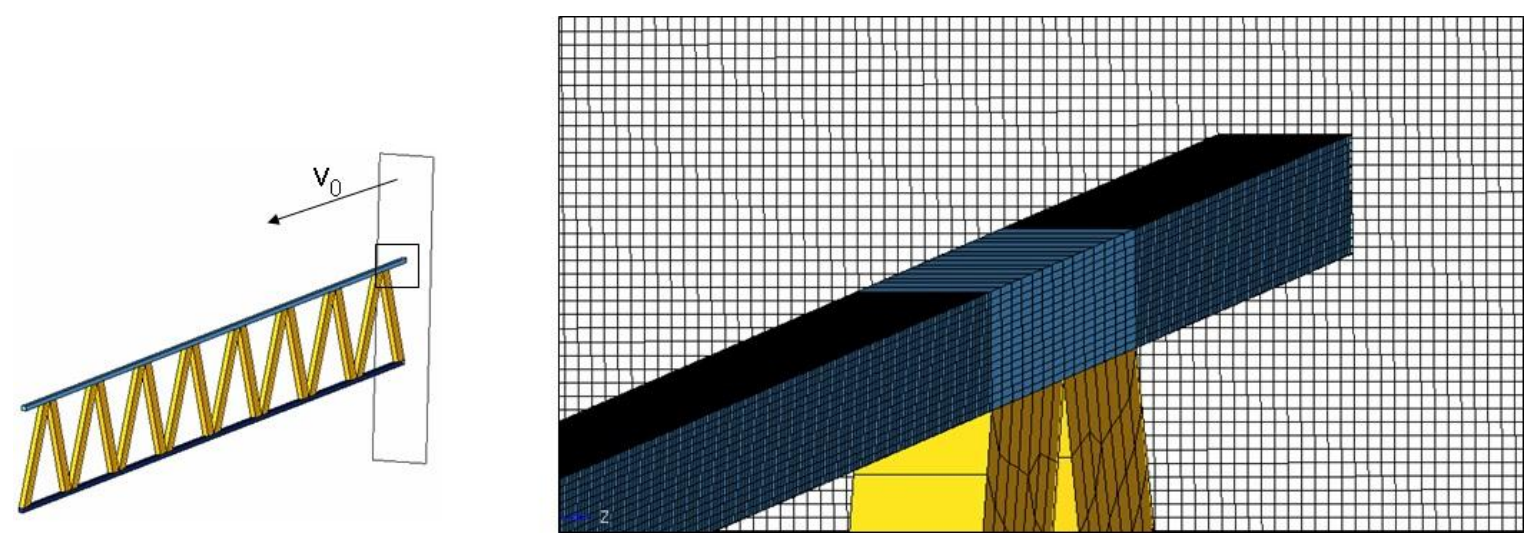

Figure 2.12: A constant velocity imposed on the front end of a sandwich column by a rigid plate.

The compression is imposed by a rigid plate, displaced at a constant velocity, V. To define the interaction between the rigid plate and the contacted end (front end) of sandwich columns, "TIED" contact option ${ }^{3}$ is used in the dynamic simulations as shown in Fig. 2.12. The other end (back end) is completely fixed.

Moreover, a general contact algorithm is used for contact between members including core-to-core, face-to-face, and face-to-core. However, such contact events are not observed for the velocity range considered in this chapter. To realize lateral constraints due to the large width dimension of sandwich panel columns, symmetry conditions on the lateral sides are imposed.

With respect to the monolithic solid columns, the same material models and boundary and loading conditions with conventional shell elements (S4R) are used for the FEA.

Finally, the dynamic FE simulations for monolithic solid columns and sandwich columns are conducted employing ABAQUS/Explicit on double-precision basis.

${ }^{3}$ As such structures employ end connectors with inserts in practice [66], the top and bottom faces at the front end do not slide individually on the rigid plate. 


\subsection{RESULTS AND DISCUSSION}

The semi-analytical models are validated by comparison with FE simulations of monolithic solid columns and corrugated core sandwich columns of various geometric dimensions with varied geometric imperfections under several applied velocities as shown in Table 2.2. In this section, some of the results will be presented by selecting one

Table 2.2. The geometric dimensions of columns for the global buckling analytical model validation.

\begin{tabular}{|c|c|c|c|c|c|c|c|c|}
\hline $\begin{array}{c}\text { Type of sandwich } \\
\text { columns }\end{array}$ & $\begin{array}{c}\mathrm{h} \\
(\mathrm{mm}) \\
\end{array}$ & $\begin{array}{c}\mathrm{t} \\
(\mathrm{mm}) \\
\end{array}$ & $\begin{array}{c}1 \\
(\mathrm{~mm})\end{array}$ & $\begin{array}{l}\mathrm{W} \\
\left({ }^{\circ}\right) \\
\end{array}$ & $\begin{array}{c}\bar{\rho} \\
(\%) \\
\end{array}$ & $\begin{array}{c}\mathrm{L} \\
(\mathrm{mm}) \\
\end{array}$ & $\xi$ & $\begin{array}{c}V \\
(\mathrm{~m} / \mathrm{s}) \\
\end{array}$ \\
\hline $\begin{array}{c}\mathrm{SS} 304 \\
\text { (perpendicular-to- } \\
\text { corrugation) }\end{array}$ & 3.75 & 1.5 & 25 & 45 & 12 & 707 & $0.05 \mathrm{c}$ & $\begin{array}{c}1,5 \text { (Rate- } \\
\text { dependent) } \\
1,5 \text { (Rate- } \\
\text { independent) }\end{array}$ \\
\hline \multirow{3}{*}{$\begin{array}{c}\text { SS304 } \\
\text { (parallel-to- } \\
\text { corrugations) }\end{array}$} & 2.5 & 1.5 & 25 & 45 & 12 & $\begin{array}{l}707 \\
1404\end{array}$ & $0.05 \mathrm{c}$ & $\begin{array}{c}1,5 \text { (Rate- } \\
\text { dependent) } \\
1,5 \text { (Rate- } \\
\text { independent) }\end{array}$ \\
\hline & 3.75 & 1.5 & 25 & 45 & 12 & $\begin{array}{l}707 \\
1404\end{array}$ & $0.05 \mathrm{c}$ & $\begin{array}{c}1,5 \text { (Rate- } \\
\text { dependent) } \\
1,5 \text { (Rate- } \\
\text { independent) }\end{array}$ \\
\hline & 3.75 & 0.675 & 25 & 45 & 5.33 & $\begin{array}{l}707 \\
1404\end{array}$ & $0.05 \mathrm{c}$ & $\begin{array}{c}1,5 \text { (Rate- } \\
\text { dependent) } \\
1,5 \text { (Rate- } \\
\text { independent) } \\
\end{array}$ \\
\hline \multirow{2}{*}{$\begin{array}{l}\text { Al6061-T6 } \\
\text { (perpendicular-to- } \\
\text { corrugation) }\end{array}$} & 4.4 & 3.175 & 22 & 60 & 25 & 1407 & $0.01 \mathrm{c}, 0.05 \mathrm{c}, 0.1 \mathrm{c}$ & $0.5,1,5$ \\
\hline & 6.6 & 3.175 & 22 & 60 & 25 & 1407 & $0.01 \mathrm{c}, 0.05 \mathrm{c}$ & 1,5 \\
\hline \multirow{2}{*}{$\begin{array}{l}\text { Al6061-T6 } \\
\text { (parallel-to- } \\
\text { corrugations) }\end{array}$} & 2.2 & 3.175 & 22 & 60 & 25 & 1407 & $0.01 \mathrm{c}, 0.05 \mathrm{c}, 0.1 \mathrm{c}$ & $0.1,0.5,1,5$ \\
\hline & 4.4 & 3.175 & 22 & 60 & 25 & 1407 & $0.01 \mathrm{c}, 0.05 \mathrm{c}$ & $0.5,1,5$ \\
\hline \multirow{2}{*}{$\begin{array}{l}\text { A16061-T6 } \\
\text { (monolithic) }\end{array}$} & \multicolumn{5}{|c|}{$A=13.56$} & 1407 & $0.01 \mathrm{c}, 0.05 \mathrm{c}, 0.1 \mathrm{c}$ & $0.1,0.5,1,5$ \\
\hline & \multicolumn{5}{|c|}{$A=5.6762,13.56$} & 469 & $0.001 \mathrm{~mm}$ & $0.1,0.5,1,5$ \\
\hline $\begin{array}{c}\mathrm{SS} 304 \\
\text { (monolithic) }\end{array}$ & \multicolumn{5}{|c|}{$A=9.62$} & 707 & $0.05 \mathrm{c}$ & $\begin{array}{c}1,5 \text { (Rate- } \\
\text { dependent) } \\
1,5 \text { (Rate- } \\
\text { independent) }\end{array}$ \\
\hline
\end{tabular}


column from each category so that three types of columns (monolithic, perpendicular-tocorrugations, parallel-to-corrugations) have the same weight: For Al6061-T6 columns, monolithic and corrugated core sandwich columns have the same areal mass (the thickness of the monolithic column) of $\mathrm{A}=13.56 \mathrm{~mm}$. For SS304 columns, the areal mass of the columns to be presented is $A=9.62 \mathrm{~mm}$.

In order to better understand the results from the semi-analytical models and FE calculations shown in Figs 2.14 through 2.19, schematic curves for the front and back end reaction forces during elastic stress wave propagation are examined in Fig. 2.13(a). As explained in Section 2.3, reaction forces at both ends increase in a stepwise manner with every wave reflection: No increase in the back end reaction force has been observed until the incident stress wave reaches the back end $\left(\mathrm{t}<\Delta t_{e l}\right)$. At the point $\left(\mathrm{t}=\Delta t_{e l}\right)$, back end reaction force jumps up due to a wave reflection at the back end. Similarly, when the elastic stress wave reaches one end $\left(\mathrm{t}=\mathrm{n} \cdot \Delta t_{e l}\right)$, the reaction force, where the wave reflection takes places, shows a stepwise increasing pattern.

Fig. 2.13(b) shows the axial stress distributions of a column at times $t=t_{1 e}, t_{2 e}, t_{3 e}$, which are midway between every wave reflection. The wave reflection leads to an increase of the reaction force curve by $\Delta \mathrm{P}_{\text {short }}=2 A_{\text {eff }} \Delta \sigma_{e l}$. (Although the other component of the reaction force, $\Delta \mathrm{P}_{\text {long }}$, also affects the magnitude of reaction force, it is assumed to change stepwise with every wave reflection in the semi-analytical calculation because the growing rate of $\Delta \mathrm{P}_{\text {long }}$ is small compared to that of $\Delta \mathrm{P}_{\text {short }}$.)

We note in passing that the reaction curves obtained from FEM show a high frequency oscillation after wave reflections rather than idealized step functions as described in Fig. 2.13 (c). (Look over the data in Fig. 2.14 2.19.) From the data analysis 
not presented in this dissertation, approximately sine-shaped waveforms with high frequency are observed after wave reflections in the FE results of monolithic solid columns, whereas irregular oscillatory responses are found in those of sandwich columns. These high-frequency oscillations have approximately several microseconds of period (5 20 $\mu \mathrm{s}$ in cases of monolithic columns, $5 \sim 50 \mu \mathrm{s}$ in cases of sandwich columns compressed parallel-to-corrugations) although the high frequency characteristics such as

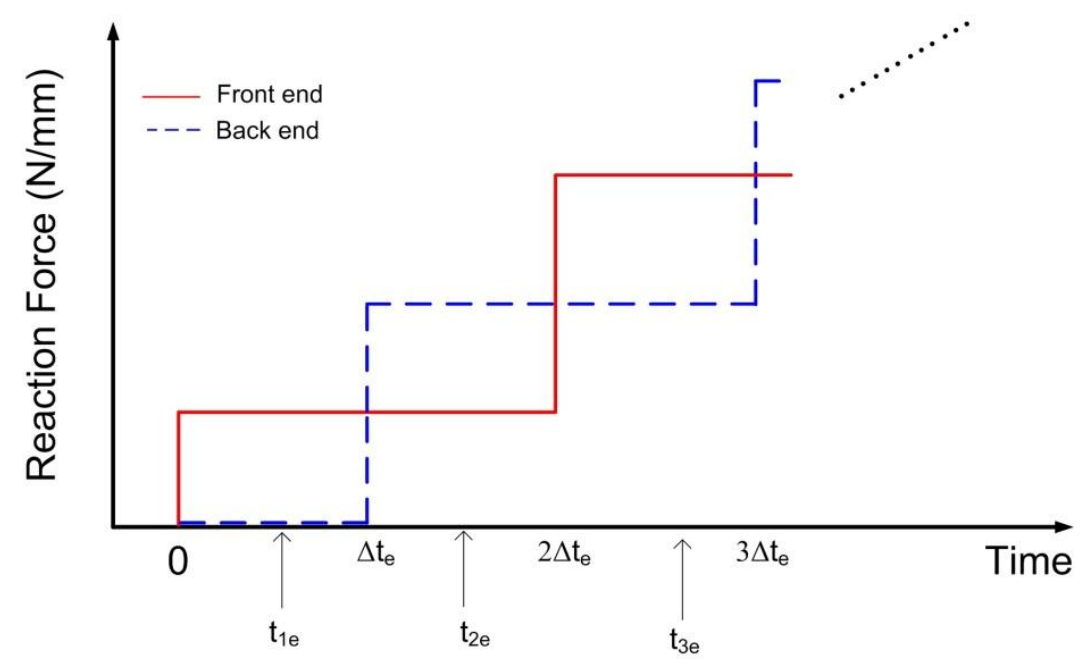

(a)
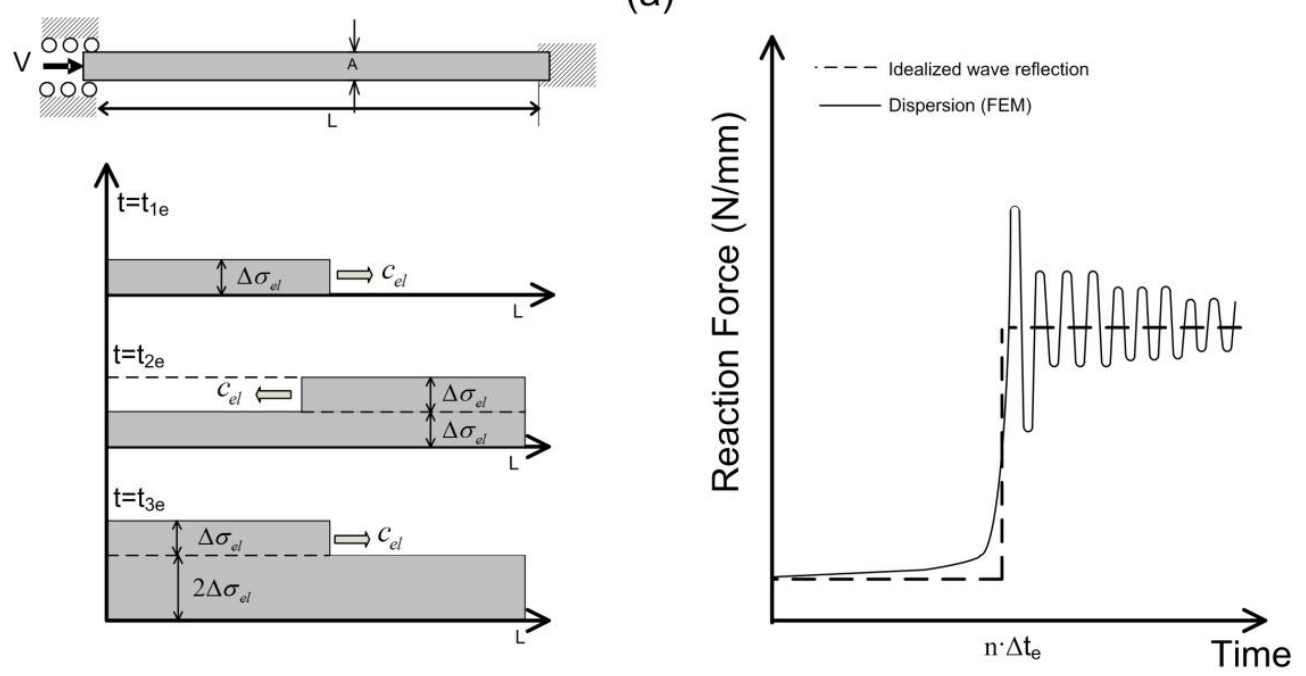

(c)

Figure 2.13: (a) Schematic curves for the front and back end reaction forces during elastic wave propagation; (b) axial stress distribution within a column at times of $t_{1 e}, t_{2 e}, t_{3 e}$; (c) idealized and FEobserved reaction force curves at a wave reflection, $t=n \cdot t_{e}$. 
the period and amplitude are affected by the specimen dimensions and the amount of compression. Physically, wave dispersion is thought to result in such an oscillatory response [104, 105]. It has been known that plates and rods with a finite thickness dimension show such behaviors even though they are elastic due to 1) the frequencydependent propagating wave speed of a harmonic wave, and 2) non-uniform axial stress distribution across the cross section as a result of the transverse inertia [104-106]. In the numerical analysis aspect, it has been reported that the high frequency response during wave propagation is highly dependent on spatial and temporal discretization, time integration method, element selection, mass matrix formulation (lumped vs. consistent mass matrices) and material damping [105,107].

This high frequency oscillation phenomenon is beyond the scope of this study, thus in-depth considerations have not been performed. Moreover, the possibility of signal aliasing or signal distortion, which might be caused by the writing frequency of the timehistory data in output files, cannot be ruled out. Therefore, it is hard to put physical significance on the high-frequency oscillatory response shown in the FE data in this section (Figs. 2.14 2.19). Nevertheless, particular responses from the FE simulations such as initial overshooting followed by decaying high-frequency oscillations accord with the reported phenomena qualitatively [104,105]. Above all, the constructed FE models are sufficient to capture the stepwise increase of reaction forces at a larger time scale than at the scale for high-frequency oscillation behavior.

\subsubsection{Monolithic Solid Columns}

Two columns are considered with several compressive velocities and imperfections: 
one column is an A16061-T6 column of thickness $\mathrm{A}=13.56 \mathrm{~mm}$ and column length $\mathrm{L}=1407 \mathrm{~mm}$ while the other is an SS304 column of thickness $\mathrm{A}=9.62 \mathrm{~mm}$ and column length $\mathrm{L}=707 \mathrm{~mm}$. An important point to note is that the monolithic columns studied will have the same mass as the corrugated core sandwich columns. This will be discussed in the following section.

Fig. 2.14 shows the dynamic response of the Al6061-T6 monolithic solid column under two applied velocities $\mathrm{V}=0.5 \mathrm{~m} / \mathrm{s}$ and $\mathrm{V}=5 \mathrm{~m} / \mathrm{s}$. Under a quasi-static load, the column is predicted to fail at a critical load of $P_{c r}^{(Q S)}=349.4(\mathrm{~N} / \mathrm{mm})$ dictated by Euler's buckling formula. The imperfections are assigned in a form of the fundamental static buckling mode, $w_{0}(x)=\frac{\xi}{2}\left(1-\cos \frac{2 \pi x}{L}\right)$, and the magnitudes of the imposed imperfections, $\xi$, are $0.1905,0.9525$, and 1.905 , which correspond to $1 \%, 5 \%$, and $10 \%$ of the core height $\mathrm{c}$ of the corrugated core sandwich columns which will be presented in the next section 2.6.2.

Figures 2.14(a) and (b) show reaction force versus time history curves of the Al6061-T6 monolithic column obtained from the FE simulations and the semi-analytical model at two applied velocities $\mathrm{V}=0.5 \mathrm{~m} / \mathrm{s}$ and $\mathrm{V}=5 \mathrm{~m} / \mathrm{s}$. The dynamic column response for the lower applied velocity, $\mathrm{V}=0.5 \mathrm{~m} / \mathrm{s}$, is sensitive to the magnitude of imperfections because the column fails below the elastic limit. The maximum peak loads are five to seven times greater than the quasi-static critical load, $P_{c r}^{(Q S)}=349.4(\mathrm{~N} / \mathrm{mm})$. For $\mathrm{V}=5 \mathrm{~m} / \mathrm{s}$ (Fig. 2.14(b)) it is revealed that elastic stress wave reflection occurred only four times prior to elastic-plastic wave propagation around $\mathrm{t}=1000 \mu \mathrm{s}$, and that the column fails during elastic-plastic stress wave propagation. 


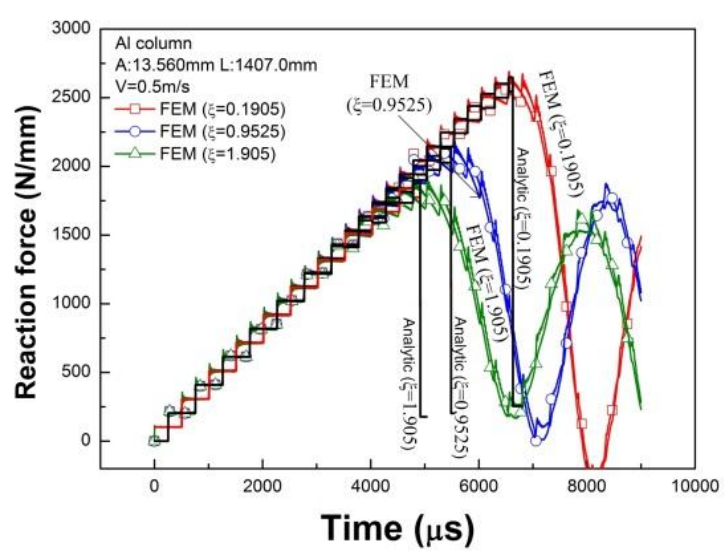

(a) $\mathrm{V}=0.5 \mathrm{~m} / \mathrm{s}$

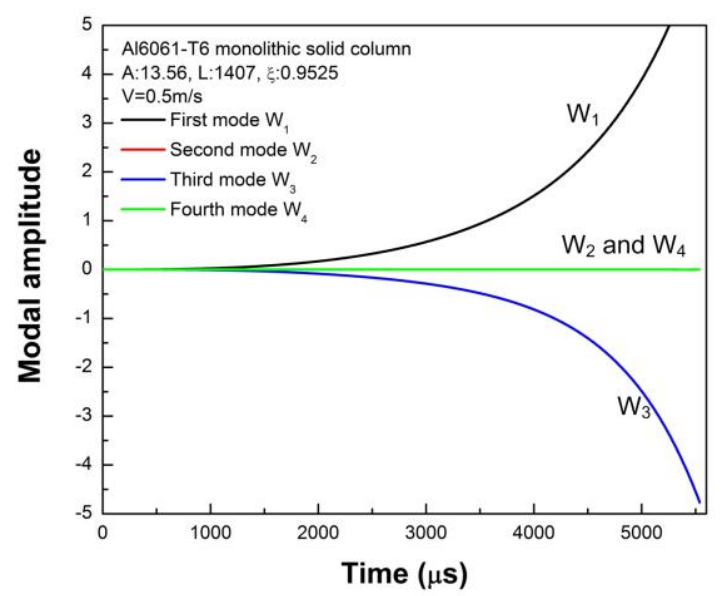

(c) $\mathrm{V}=0.5 \mathrm{~m} / \mathrm{s}$

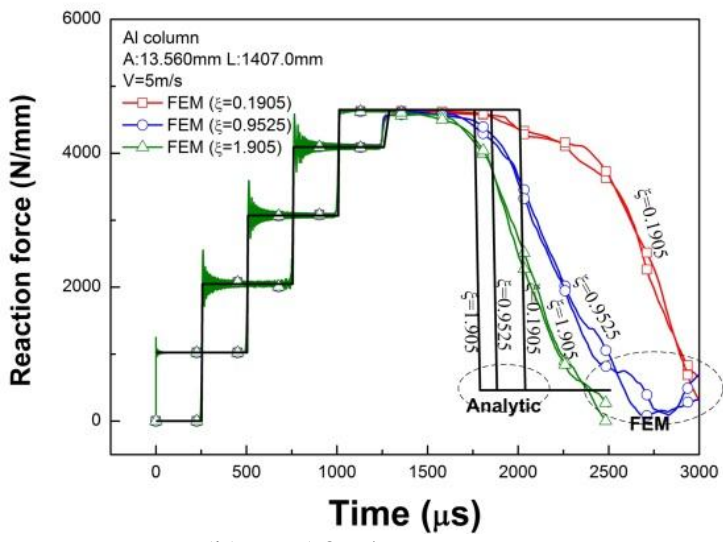

(b) $\mathrm{V}=5.0 \mathrm{~m} / \mathrm{s}$

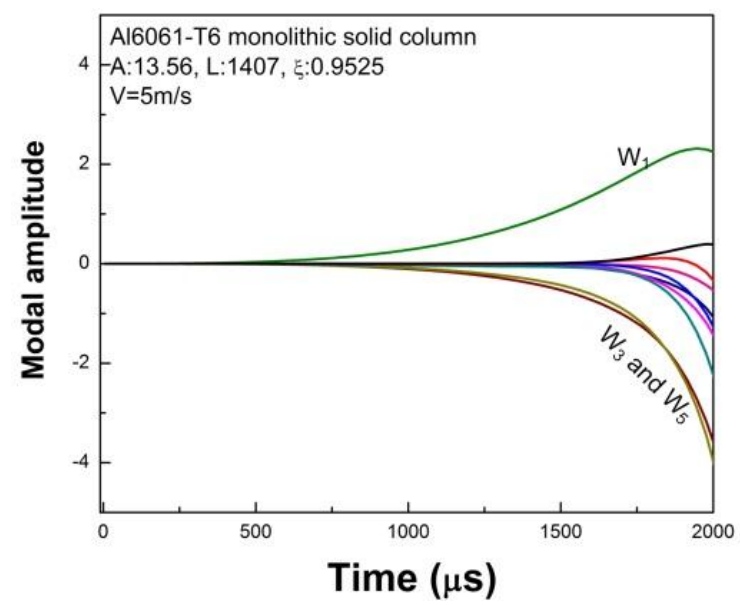

(d) $V=5.0 \mathrm{~m} / \mathrm{s}$

Figure 2.14: Dynamic response of an Al6061-T6 monolithic solid column of A=13.56mm, L=1407mm with $\xi=0.1905,0.9525,1.905$ under applied compressive velocities of 0.5 and $5 \mathrm{~m} / \mathrm{s}$ obtained from FEM and the semi-analytical model: (a)(b) Reaction force versus time history curves for $\mathrm{V}=0.5 \mathrm{~m} / \mathrm{s}$ and $5 \mathrm{~m} / \mathrm{s}$; (c)(d) The growth of modal amplitudes of the column with an imperfection magnitude $\xi=0.9525$ for $\mathrm{V}=0.5 \mathrm{~m} / \mathrm{s}$ and $5 \mathrm{~m} / \mathrm{s}$.

Figures 2.14(c) and (d) show the growths of modal amplitudes for the column with one of the three considered imperfection magnitudes, $\xi=0.9525$, calculated from the semianalytical model under $\mathrm{V}=0.5$ and $5 \mathrm{~m} / \mathrm{s}$, respectively. For $\mathrm{V}=0.5 \mathrm{~m} / \mathrm{s}$, there are only two growing modes, the first and third modes, while the other modes remain bounded as shown in Fig. 2.14(c). Fig. 2.14(d) shows the lowest ten modes among eighteen employed modes in the semi-analytical model. Before $t=1500 \mu$ s, the only growing modes are the first, third and fifth modes. However, after that, most of modes are simultaneously 
activated after inelastic deformation in the column begins. This is because the tangent modulus is significantly less than Young's modulus.

The dynamic response of the SS304 monolithic solid column for two applied velocities, $V=1$ and $5 \mathrm{~m} / \mathrm{s}$ are shown in Fig. 2.15. The column has the same mass as the SS304 corrugated core sandwich columns of $h=3.75 \mathrm{~mm}, \mathrm{t}=1.5 \mathrm{~mm}, \mathrm{l}=25.0 \mathrm{~mm}, \mathrm{w}=45^{\circ}$, $\bar{\rho}=12 \%, \mathrm{~L}=707 \mathrm{~mm}$ which will be presented in the next Section 2.6.2. The lowest static buckling mode with $\xi=0.884$, is considered for geometric imperfections. The column has a critical load at $P_{c r}^{(Q S)}=1485.9(\mathrm{~N} / \mathrm{mm})$ dictated by Euler's buckling formula [28]. The analysis also includes the rate-independent FE calculation and analytical results obtained by suppressing the material-rate dependence of SS304 in order to investigate the material strain-rate effect.

As shown in Figures 2.15(a) and (b), the rate-dependent column resisted loads much higher than the static critical load $\left(P_{c r}^{(Q S)}=1485.9(\mathrm{~N} / \mathrm{mm})\right)$ under the two applied velocities $\mathrm{V}=1,5 \mathrm{~m} / \mathrm{s}$. Consequently, the columns failed in the plastic region.

In addition, the FE and semi-analytical calculations without considering material strainrate dependence are superimposed in the graphs. A stepwise increase in the hardening region is exhibited in the rate-independent FE simulations, which is clear evidence of elastic-plastic stress wave propagation. In contrast to the rate-independent simulations, the reaction force in the plastic region of the strain-rate dependent FE analysis increases smoothly.

In the semi-analytical model, strain-rate dependence was taken into account by considering the rate-dependent column material as a rate-independent material with elevated yield strength and adjusted hardening moduli as stated in Section 2.4.1. It is 


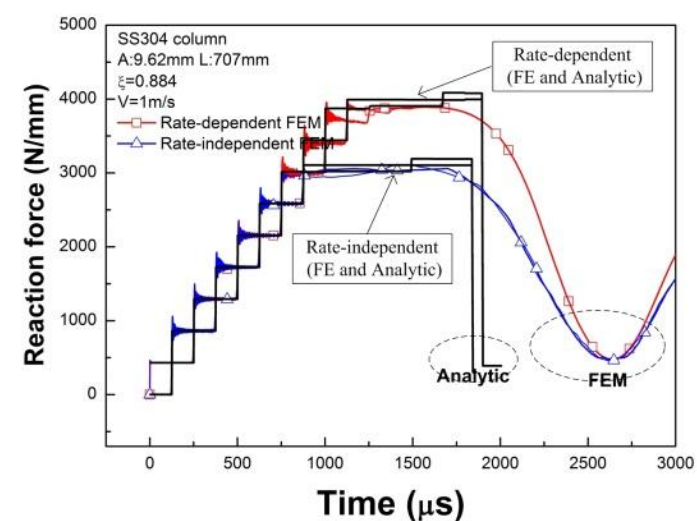

(a) $\mathrm{V}=1.0 \mathrm{~m} / \mathrm{s}$

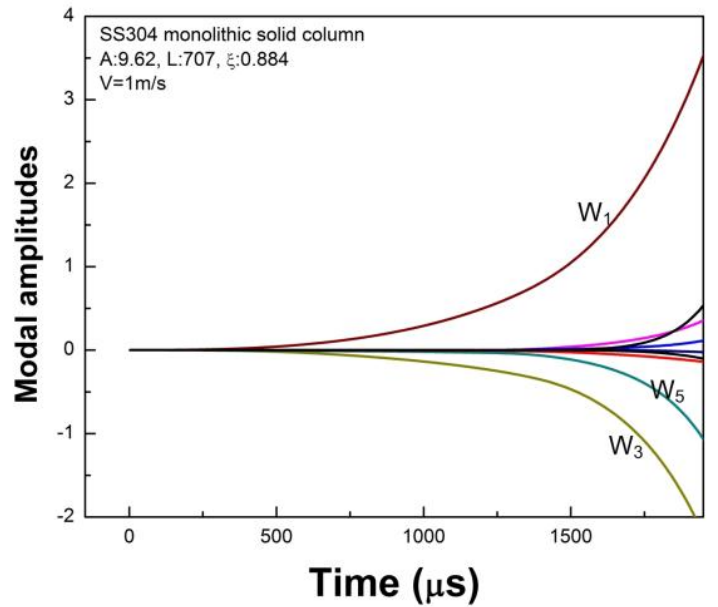

(c) $\mathrm{V}=1.0 \mathrm{~m} / \mathrm{s}$

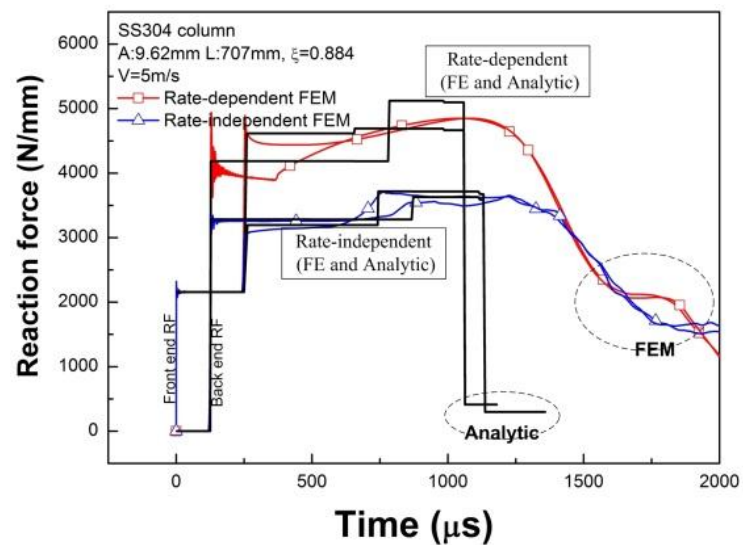

(b) $\mathrm{V}=5.0 \mathrm{~m} / \mathrm{s}$

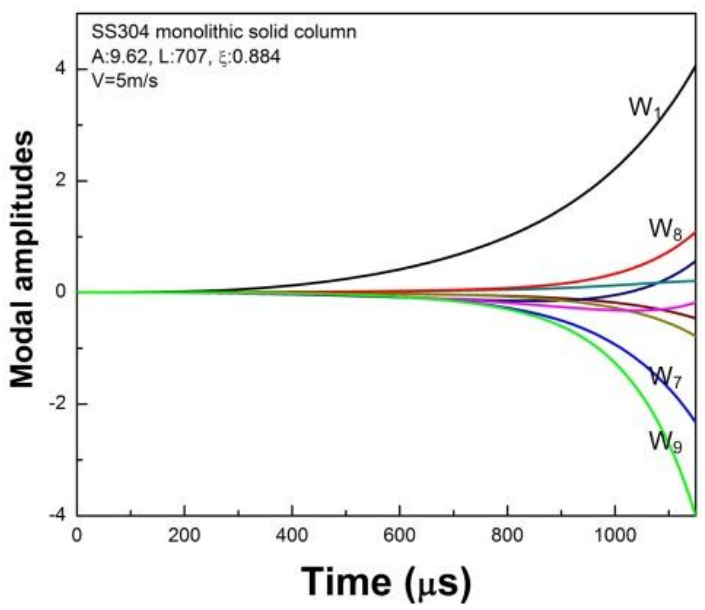

(d) $\mathrm{V}=5.0 \mathrm{~m} / \mathrm{s}$

Figure 2.15: Dynamic response of a SS304 monolithic solid column of A=9.62mm, L=707mm with $\xi=0.884$ under compression velocities of 1 and $5 \mathrm{~m} / \mathrm{s}$ obtained from FEM and the semi-analytic model: (a)(b) Reaction force versus time history curves for $\mathrm{V}=1.0 \mathrm{~m} / \mathrm{s}$ and $5 \mathrm{~m} / \mathrm{s}$; (c)(d) The growths of modal amplitudes of the rate-dependent column with an imperfection magnitude $\xi=0.884$ for $\mathrm{V}=1.0 \mathrm{~m} / \mathrm{s}$ and $5 \mathrm{~m} / \mathrm{s}$.

demonstrated that the semi-analytical model successfully approximates the strain-rate dependent dynamic response through Perrone's approximation [75].

Figures 2.15(c) and (d) show the growths of modal amplitudes for the column under $\mathrm{V}=1$ and $5 \mathrm{~m} / \mathrm{s}$ calculated from the semi-analytical model, respectively. For $\mathrm{V}=1 \mathrm{~m} / \mathrm{s}$, there are only two growing modes during elastic column deformation as shown in Fig. 2.15(c). However, after $\mathrm{t}=1000 \mu \mathrm{s}$, the other eigenmodes are growing from lower to higher modes. Fig. $2.15(\mathrm{~d})$ shows the lowest ten modes for the $\mathrm{V}=5 \mathrm{~m} / \mathrm{s}$ case. Similarly to 
the Al6061-T6 monolithic solid column under $\mathrm{V}=5 \mathrm{~m} / \mathrm{s}$, most of the modes are simultaneously activated after inelastic deformation in the column begins as the tangent modulus is significantly lower than Young's modulus.

\subsubsection{Corrugated Core Sandwich Columns}

In this section, the dynamic response of corrugated core sandwich columns compressed perpendicular-to-corrugations and parallel-to-corrugations obtained from the semi-analytical model is compared against the FE results. The Al6061-T6 and SS304 sandwich columns have the same weight as the monolithic columns in Section 2.6.1.

Fig. 2.16 shows the dynamic response of an Al6061-T6 sandwich column compressed perpendicular-to-corrugations under two applied velocities $\mathrm{V}=0.5 \mathrm{~m} / \mathrm{s}$ and $5 \mathrm{~m} / \mathrm{s}$. The geometry of the column has face sheet thickness $\mathrm{h}=4.4 \mathrm{~mm}$, column length $\mathrm{L}=1407 \mathrm{~mm}$, corrugation angle $\mathrm{w}=60^{\circ}$, core ligament thickness $\mathrm{t}=3.175 \mathrm{~mm}$, and core ligament length $1=22.0 \mathrm{~mm}$. The geometric dimensions of the corrugated core result in a core relative density, $\bar{\rho}=25 \%$. The column is assumed to have geometric curvature

imperfections in a form of the first eigen-mode $w_{0}(x)=\frac{\xi}{2}\left(1-\cos \frac{2 \pi x}{L}\right)$. This column would collapse at $\mathrm{P}_{\mathrm{cr}}=2082 \mathrm{~N} / \mathrm{mm}$ under static loading [28].

Figures 2.16(a) and (b) show reaction force versus time history curves of the Al6061-T6 corrugated core sandwich column under the axial velocities of $V=0.5 \mathrm{~m} / \mathrm{s}$ and $5 \mathrm{~m} / \mathrm{s}$. The response with respect to various imperfection magnitudes of $1 \%, 5 \%$, and $10 \%$ of core height, c, are obtained. Under $\mathrm{V}=0.5 \mathrm{~m} / \mathrm{s}$, the column of imperfection $\xi=0.05 \mathrm{c}$ $(\xi=0.9525)$ and $\xi=0.1 \mathrm{c}(\xi=1.905)$ fail in the elastic region while the column having an imperfection magnitude of $\xi=0.01 \mathrm{c}(\xi=0.1905)$ fails in the plastic region. Therefore, it 
appears to be imperfection-sensitive because it failed in the elastic region (i.e., the difference of peak reaction forces between $\xi=0.01 \mathrm{c}$ and $\xi=0.1 \mathrm{c}$ is $20 \%$ ). However, under $\mathrm{V}=5 \mathrm{~m} / \mathrm{s}$ all failures occur in the plastic region because the effects of transverse inertia delay the buckling motion. However, many buckling modes are simultaneously activated after inelastic deformation begins to appear due to significantly lowered tangent stiffness compared to the elastic stiffness. Compared to the monolithic solid columns, the sandwich columns in this in-plane loading orientation have a less effective area to support the axial compressive load even though they are of the same weight; the core does not contribute to the in-plane load support. Accordingly, the reaction force associated with yielding is lower $(\sim 3000 \mathrm{~N} / \mathrm{mm})$ than the one in monolithic solid columns $(\sim 4600 \mathrm{~N} / \mathrm{mm})$.

Figures 2.16(c) and (d) show the growths of modal amplitudes of the sandwich column with one imperfection magnitude $\xi=0.05 \mathrm{c}$ obtained from the semi-analytical model. For clarity, the first four modal amplitudes are included in Fig. 2.16(c). For $\mathrm{V}=0.5 \mathrm{~m} / \mathrm{s}$, the first mode predominates and leads to column failure while the other modes are bounded. For $\mathrm{V}=5 \mathrm{~m} / \mathrm{s}$, the first mode is the only growing mode below the elastic limit, however, then all the other modes become unbounded after the column starts to deform inelastically. In Figs. 2.16 (e)-(h), the deformed profiles from the FE simulations and the semi-analytical model near the time of failure are compared.

Fig. 2.17 shows the dynamic response of the SS304 corrugated core sandwich column compressed perpendicular-to-corrugations under two applied velocities $\mathrm{V}=1 \mathrm{~m} / / \mathrm{s}$ and $5 \mathrm{~m} / \mathrm{s}$. The geometry of the column is characterized by face sheet thickness $\mathrm{h}=3.75 \mathrm{~mm}$, column length $\mathrm{L}=707 \mathrm{~mm}$, corrugation angle $\mathrm{w}=45^{\circ}$, core ligament thickness 
$\mathrm{t}=1.5 \mathrm{~mm}$, and core ligament length $\mathrm{l}=25.0 \mathrm{~mm}$. The geometric dimensions of the core result in a core relative density, $\bar{\rho}=12 \%$. Consequently, the column has an equivalent mass as the SS304 monolithic column in the previous section. This column would fail at $\mathrm{P}_{\mathrm{cr}}=2718 \mathrm{~N} / \mathrm{mm}$ under quasi-static loading.

Figs. 2.17(a) and (b) shows the reaction force versus time history curves of the SS304 corrugated core sandwich column under V=1m/s and $5 \mathrm{~m} / \mathrm{s}$ obtained from the FE simulations and the semi-analytical models. The magnitude of imperfection, assigned in a form of the lowest buckling mode, is $5 \%$ of the core height $\mathrm{c}$. To evaluate the effect of strain-rate dependence, the FE simulations without the material rate dependence and the corresponding semi-analytical results are superposed in the same graphs.

The semi-analytical model predicts the dynamic response at $\mathrm{V}=1 \mathrm{~m} / \mathrm{s}$ to reasonable accuracy as shown in Fig. 2.17(a). However, as shown in Fig. 2.17(b), there is a significant discrepancy between the FE and semi-analytical results for the response at $\mathrm{V}=5 \mathrm{~m} / \mathrm{s}$. This can be accounted for by investigating the FE deformed profiles in Figures 2.17(d) and (e). The deformed profiles obtained from the FE simulation demonstrated that the column fails by face wrinkling under the high velocity $\mathrm{V}=5 \mathrm{~m} / \mathrm{s}$ whereas it fails by global buckling motion under $\mathrm{V}=1.0 \mathrm{~m} / \mathrm{s}$. Although this will be discussed in another chapter in detail, the rate of growth of buckling modes associated with face wrinkling is much faster than that associated with the global buckling motion because the wave length in face wrinkling failure mode is much shorter. 


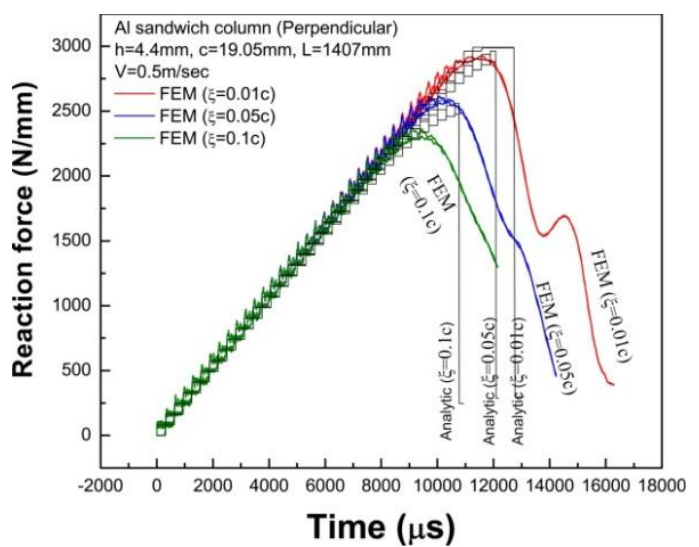

(a) $\mathrm{V}=0.5 \mathrm{~m} / \mathrm{s}(\xi=0.01 \mathrm{c}, 0.05 \mathrm{c}, 0.1 \mathrm{c})$

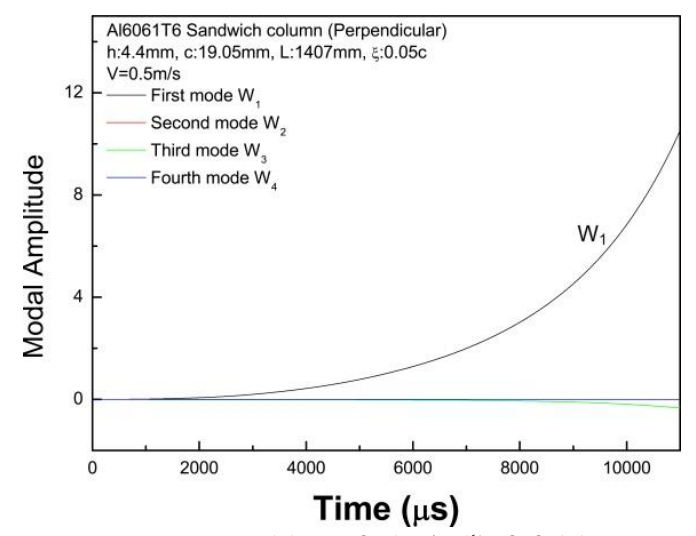

(c) $\mathrm{V}=0.5 \mathrm{~m} / \mathrm{s}(\xi=0.05 \mathrm{c})$

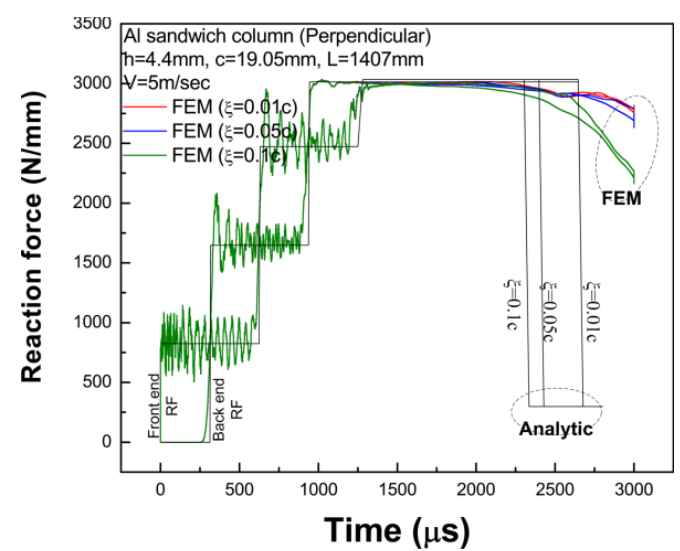

(b) $\mathrm{V}=5.0 \mathrm{~m} / \mathrm{s}(\xi=0.01 \mathrm{c}, 0.05 \mathrm{c}, 0.1 \mathrm{c})$

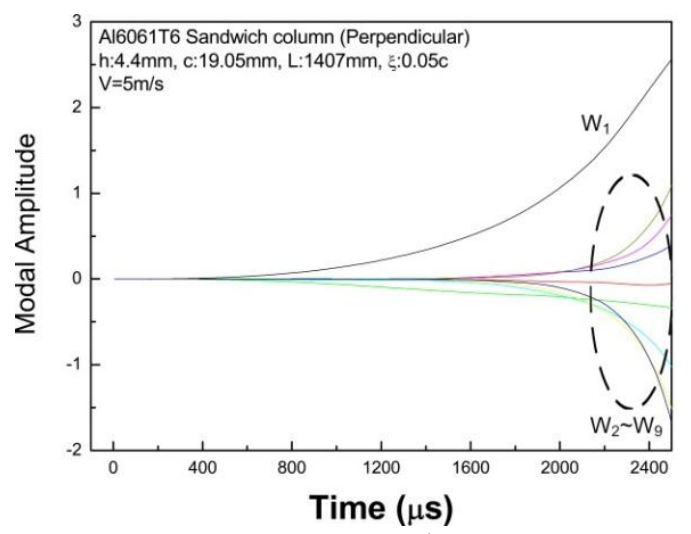

(d) $\mathrm{V}=5 \mathrm{~m} / \mathrm{s}(\xi=0.05 \mathrm{c})$

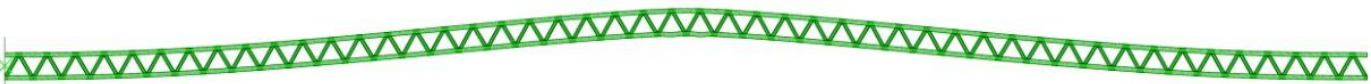

(e) $\mathrm{V}=0.5 \mathrm{~m} / \mathrm{s}(\xi=0.05 \mathrm{c})$ at $\mathrm{t}=10500 \mu \mathrm{s}$ obtained from FEM (Mag 5)

\section{Whmmwn}

(f) $\mathrm{V}=5 \mathrm{~m} / \mathrm{s}(\xi=0.05 \mathrm{c})$ at $\mathrm{t}=2500 \mu \mathrm{s}$ obtained from FEM (Mag 5)

(g) $\mathrm{V}=0.5 \mathrm{~m} / \mathrm{s}(\xi=0.05 \mathrm{c})$ at $\mathrm{t}=10600 \mu \mathrm{s}$ obtained from the semi-analytical model

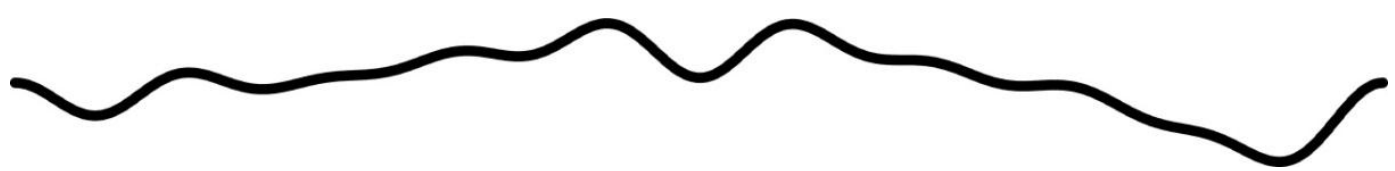

(h) $\mathrm{V}=5.0 \mathrm{~m} / \mathrm{s}(\xi=0.05 \mathrm{c})$ at $\mathrm{t}=2260 \mu \mathrm{s}$ obtained from the semi-analytical model

Figure 2.16: Dynamic response of an Al6061-T6 corrugated core sandwich columns (perpendicular-tocorrugations) under $\mathrm{V}=0.5$ and $5 \mathrm{~m} / \mathrm{s}$ obtained from FEM and the semi-analytical model $(\mathrm{h}=4.4 \mathrm{~mm}$, $\mathrm{t}=3.175 \mathrm{~mm}, \mathrm{l}=22.0 \mathrm{~mm}, \mathrm{w}=60^{\circ}, \bar{\rho}=25 \%, \mathrm{~L}=1407 \mathrm{~mm}$ ): (a)(b) Reaction force versus time history curves for $\mathrm{V}=0.5 \mathrm{~m} / \mathrm{s}$ and $5 \mathrm{~m} / \mathrm{s}$; (c)(d) The growth of the lowest four modal amplitudes of the column with an imperfection magnitude $\xi=0.05 \mathrm{c}$ for $\mathrm{V}=0.5 \mathrm{~m} / \mathrm{s}$ and $5 \mathrm{~m} / \mathrm{s}$; (e)(f) Deformed profiles of the column with an imperfection magnitude $\xi=0.05 \mathrm{c}$ for $\mathrm{V}=0.5 \mathrm{~m} / \mathrm{s}$ and $5 \mathrm{~m} / \mathrm{s}$ near the time-of-failure ( $\mathrm{t}=10500$ and $2500 \mu \mathrm{s}$ ) obtained from the FE simulations (Mag 5); (g)(h) Deformed profiles of the column with an imperfection magnitude $\xi=0.05 \mathrm{c}$ for $\mathrm{V}=0.5 \mathrm{~m} / \mathrm{s}$ and $5 \mathrm{~m} / \mathrm{s}$ the time-of-failure ( $\mathrm{t}=10600$ and $2260 \mu \mathrm{s})$ obtained from the semi-analytical model. 


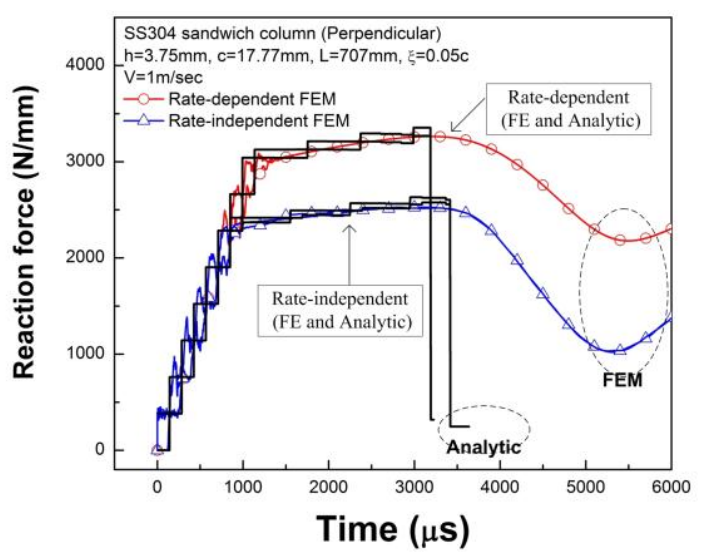

(a) $\mathrm{V}=1 \mathrm{~m} / \mathrm{s}(\zeta=0.05 \mathrm{c})$

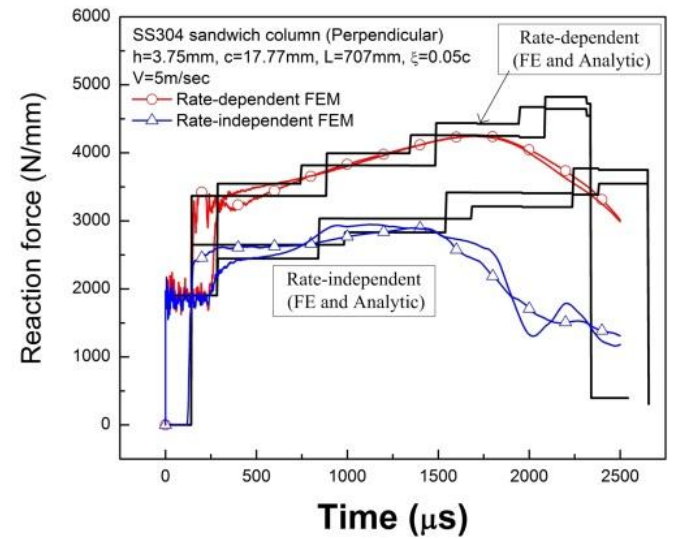

(b) $\mathrm{V}=5 \mathrm{~m} / \mathrm{s}(\zeta=0.05 \mathrm{c})$

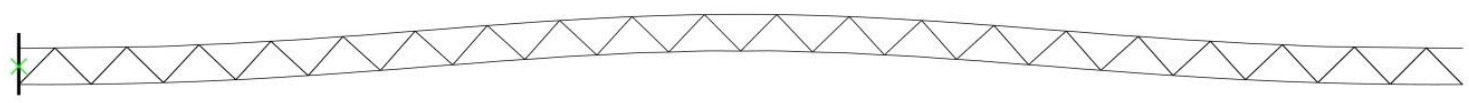

(c) $\mathrm{V}=1 \mathrm{~m} / \mathrm{s}(\zeta=0.05 \mathrm{c})$ at $\mathrm{t}=4000 \mu$ s obtained from FEM (MAG 5)

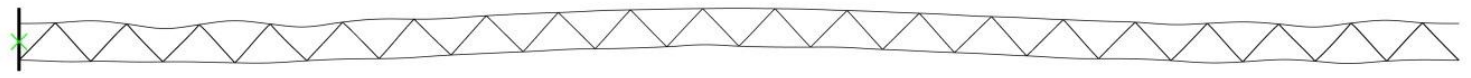

(d) $\mathrm{V}=5 \mathrm{~m} / \mathrm{s}(\zeta=0.05 \mathrm{c})$ at $\mathrm{t}=1875 \mu$ s obtained from rate-dependent FEM (MAG 2)

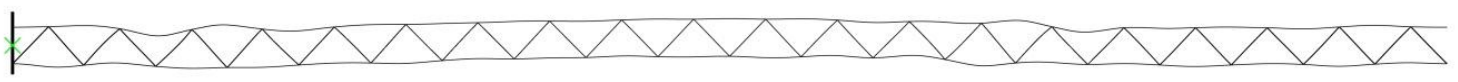

(e) $\mathrm{V}=5 \mathrm{~m} / \mathrm{s}(\zeta=0.05 \mathrm{c})$ at $\mathrm{t}=1500 \mu \mathrm{s}$ obtained from rate-independent FEM (MAG 2)

Figure 2.17: Dynamic response of a SS304 corrugated core sandwich column (compressed perpendicularto-corrugations) under applied compression velocities of $\mathrm{V}=1$ and $5 \mathrm{~m} / \mathrm{s}$ obtained from FEM and the semianalytical model $\left(\mathrm{h}=3.75 \mathrm{~mm}, \mathrm{t}=1.5 \mathrm{~mm}, \mathrm{l}=25.0 \mathrm{~mm}, \mathrm{w}=45^{\circ}, \bar{\rho}=12 \%, \mathrm{~L}=707 \mathrm{~mm}\right)$ : (a)(b) Reaction force versus time history curves for $\mathrm{V}=1 \mathrm{~m} / \mathrm{s}$ and $5 \mathrm{~m} / \mathrm{s}$; (c)(d) Deformed profiles of the rate-dependent column with an imperfection magnitude $\xi=0.05 \mathrm{c}$ for $\mathrm{V}=1 \mathrm{~m} / \mathrm{s}$ and $5 \mathrm{~m} / \mathrm{s}$ near the time-of-failure $(\mathrm{t}=4000$ and $1875 \mu$ s) obtained from the FE simulations (Mag 2 respectively); (e) Deformed profile of the rateindependent column with an imperfection magnitude $\xi=0.05 \mathrm{c}$ for $\mathrm{V}=5 \mathrm{~m} / \mathrm{s}$ near the time-of-failure $(\mathrm{t}=1500 \mu \mathrm{s})$.

On the other hand, the semi-analytical model is validated for the dynamic response of the corrugated core sandwich columns compressed parallel-to-corrugations.

Fig. 2.18 shows the dynamic response of an Al6061-T6 corrugated core sandwich column compressed parallel-to-corrugations under two applied compressive velocities $\mathrm{V}=0.5 \mathrm{~m} / \mathrm{s}$ and $5 \mathrm{~m} / \mathrm{s}$. The Al6061-T6 sandwich column has the same geometric 
dimensions as the Al6061-T6 corrugated core sandwich column for perpendicular-tocorrugations: $\mathrm{h}=4.4 \mathrm{~mm}, \mathrm{t}=3.175 \mathrm{~mm}, \mathrm{l}=22.0 \mathrm{~mm}, \mathrm{w}=60^{\circ}, \bar{\rho}=0.25, \mathrm{~L}=1407 \mathrm{~mm}$. The column is predicted to fail by elastic global buckling at a static critical load of $\mathrm{P}_{\mathrm{cr}}=2274(\mathrm{~N} / \mathrm{mm})$. Geometric imperfections with three different magnitudes $\xi=0.01 \mathrm{c}$, $0.05 \mathrm{c}$ and $0.1 \mathrm{c}$ are imposed in the form of elastic static buckling mode. As shown in Fig. 2.18(a), the column fails by elastic global buckling at $\mathrm{V}=0.5 \mathrm{~m} / \mathrm{s}$ showing the imperfection-sensitive response. On the other hand, the buckling deformation at $\mathrm{V}=5 \mathrm{~m} / \mathrm{s}$ has been resisted until material yielding occurs as shown in Fig. 2.18(b). In this loading orientation, the core plates of corrugated core sandwich columns support axial loads so that the load associated with yielding is similar to the monolithic solid column of equivalent mass, and greater than the sandwich column loaded perpendicular-tocorrugations.

Figures 2.18(c) and (d) show the growths of modal amplitudes of the sandwich column with imperfection magnitude $\xi=0.05$ c for the two velocity cases $V=0.5 \mathrm{~m} / \mathrm{s}$ and $\mathrm{V}=5 \mathrm{~m} / \mathrm{s}$, respectively. For $\mathrm{V}=0.5 \mathrm{~m} / \mathrm{s}$, the lowest buckling mode continuously grows and leads to failure while the other modes are dormant. However, higher modes are activated at the same time of yielding due to suddenly lowered material stiffness at V=5m/s. In Fig. 2.18(e)-(h), the deformed profiles calculated from the FE simulations and the semianalytical model are illustrated near the time of load drop. The analytical model predicts the failure profile for $\mathrm{V}=0.5 \mathrm{~m} / \mathrm{s}$. Moreover, the development of higher buckling mode under the high velocity of $\mathrm{V}=5 \mathrm{~m} / \mathrm{s}$ is predicted by the analytical model even though the actual deformation shape is not accurately captured. 


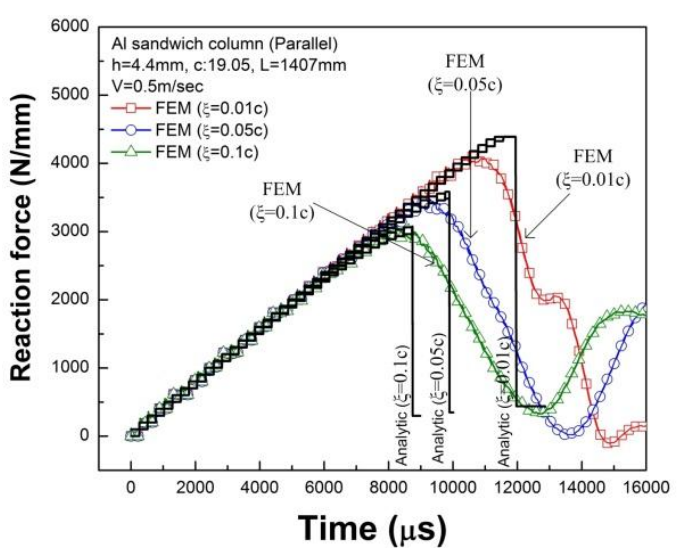

(a) $\mathrm{V}=0.5 \mathrm{~m} / \mathrm{s}$

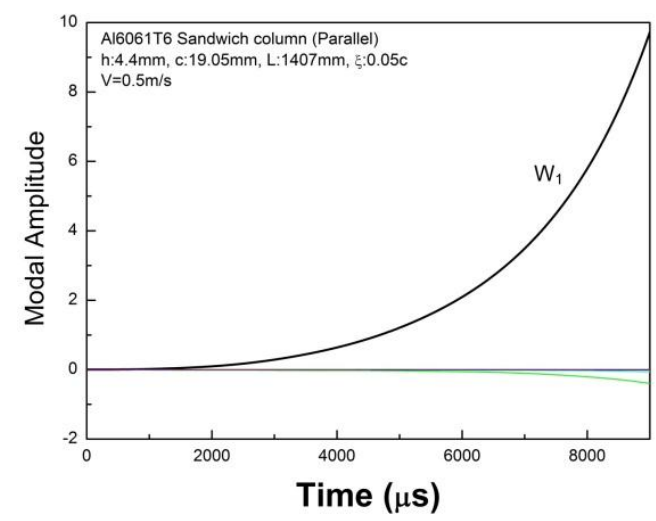

(c) $\mathrm{V}=0.5 \mathrm{~m} / \mathrm{s}(\xi=0.05 \mathrm{c})$

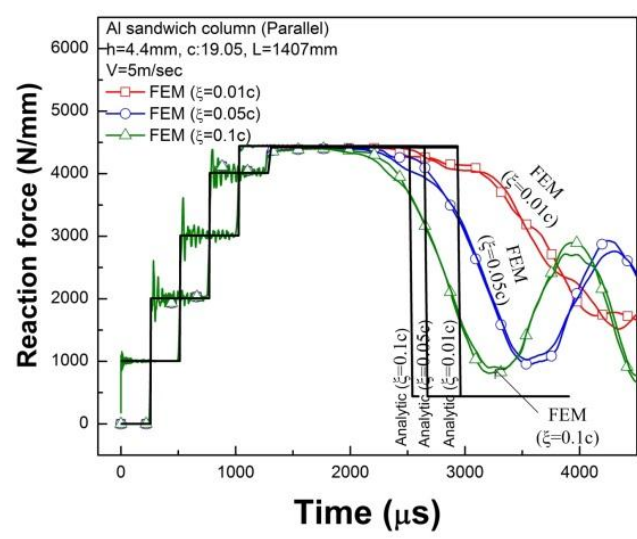

(b) $\mathrm{V}=5.0 \mathrm{~m} / \mathrm{s}$

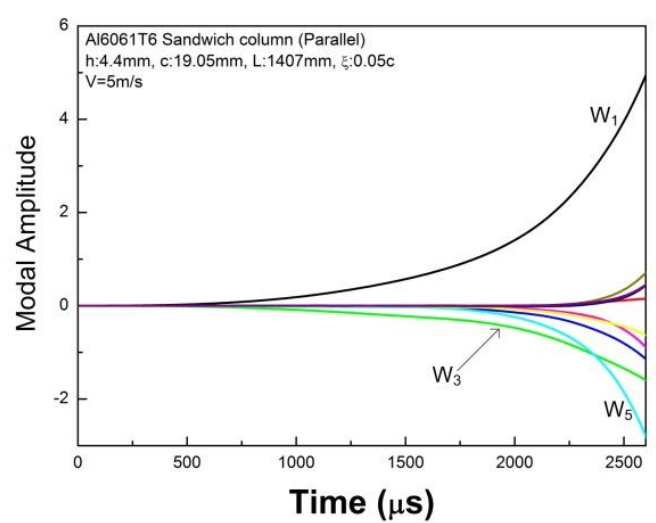

(d) $\mathrm{V}=5 \mathrm{~m} / \mathrm{s}(\xi=0.05 \mathrm{c})$

(e) $\mathrm{V}=0.5 \mathrm{~m} / \mathrm{s}(\xi=0.05 \mathrm{c})$ at $\mathrm{t}=9000 \mu \mathrm{s}$ obtained from FEM (Mag 5)

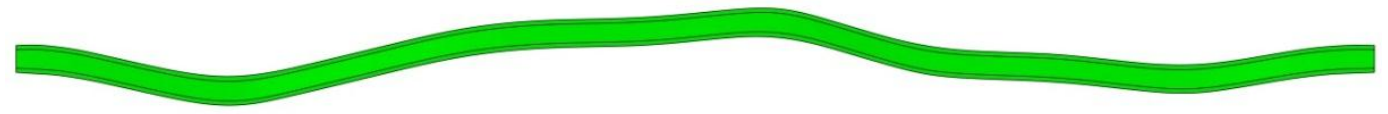

(f) $\mathrm{V}=5.0 \mathrm{~m} / \mathrm{s}(\xi=0.05 \mathrm{c})$ at $\mathrm{t}=2667 \mu$ s obtained from FEM (Mag 7)

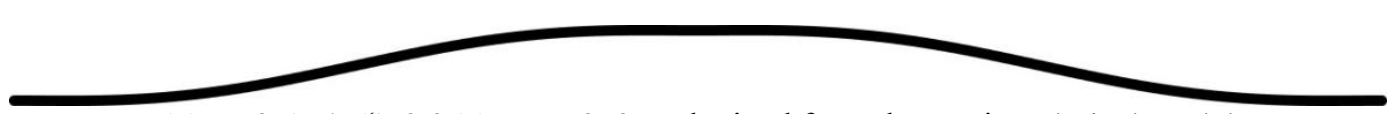

(g) $V=0.5 \mathrm{~m} / \mathrm{s}(\xi=0.05 \mathrm{c})$ at $\mathrm{t}=9060 \mu \mathrm{s}$ obtained from the semi-analytical model

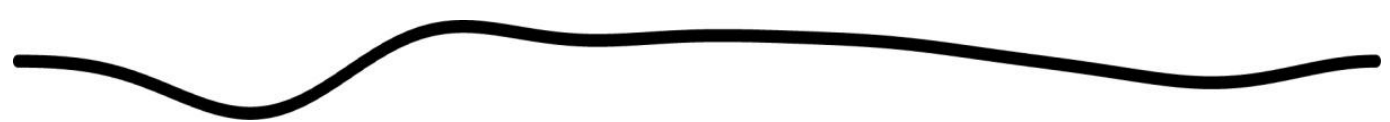

(h) $\mathrm{V}=5.0 \mathrm{~m} / \mathrm{s}(\xi=0.05 \mathrm{c})$ at $\mathrm{t}=2673 \mu$ s obtained from the semi-analytical model

Figure 2.18: Dynamic response of an Al6061-T6 corrugated core sandwich column (parallel-tocorrugations) under compression velocities of 0.5 and $5 \mathrm{~m} / \mathrm{s}\left(\mathrm{h}=4.4 \mathrm{~mm}, \mathrm{t}=3.175 \mathrm{~mm}, \mathrm{l}=22.0 \mathrm{~mm}, \mathrm{w}=60^{\circ}\right.$, $\bar{\rho}=25 \%, \mathrm{~L}=1407 \mathrm{~mm}$ ): (a)(b) Reaction force versus time history curves for $\mathrm{V}=0.5 \mathrm{~m} / \mathrm{s}$ and $5 \mathrm{~m} / \mathrm{s}$; (c)(d) The growth of modal amplitudes of the column $(\xi=0.05 \mathrm{c}$ ) for $\mathrm{V}=0.5 \mathrm{~m} / \mathrm{s}$ and $5 \mathrm{~m} / \mathrm{s}$; (e)(f) Deformed profiles of the column $(\xi=0.05 \mathrm{c}$ ) for $\mathrm{V}=0.5 \mathrm{~m} / \mathrm{s}$ and $5 \mathrm{~m} / \mathrm{s}$ near the time-of-failure ( $\mathrm{t}=9000$ and $2667 \mu \mathrm{s})$ obtained from the FE simulations (Mag 5,7); (g)(h) Deformed profiles of the column ( $\xi=0.05 \mathrm{c}$ ) for $\mathrm{V}=0.5 \mathrm{~m} / \mathrm{s}$ and $5 \mathrm{~m} / \mathrm{s}$ the time-of-failure ( $\mathrm{t}=9060$ and $2673 \mu \mathrm{s})$ obtained from the semi-analytical model. 
In Fig. 2.19, the dynamic global buckling response of an SS304 corrugated core sandwich column compressed parallel-to-corrugations is presented for two compression velocities of $\mathrm{V}=1 \mathrm{~m} / \mathrm{s}$ and $5 \mathrm{~m} / \mathrm{s}$. The column has the same geometric dimensions as the SS304 sandwich column compressed perpendicular-to-corrugations: $\mathrm{h}=3.75 \mathrm{~mm}$, $\mathrm{t}=1.5 \mathrm{~mm}, \mathrm{l}=25.0 \mathrm{~mm}, \mathrm{w}=45^{\circ}, \bar{\rho}=0.12, \mathrm{~L}=707 \mathrm{~mm}$. The static failure load is $\mathrm{P}_{\mathrm{cr}}=3295$ $\mathrm{N} / \mathrm{mm}$ dictated by macro plastic buckling failure [28]. The magnitude of geometric curvature imperfection $\zeta=0.05 \mathrm{c}$ is assigned in the form of the lowest static buckling mode. To examine the material strain-rate effect, the FE simulations suppressing the strain-rate dependence are also performed.

Figs. 2.19(a) and (b) show the reaction force versus time history curves obtained from the semi-analytical model and FE simulations for $\mathrm{V}=1.0$ and $5.0 \mathrm{~m} / \mathrm{s}$. The ratedependent FE results show elevated yield strength with smooth elastic-plastic strain hardening while the strain-hardening in the rate-independent FE results takes a form of a stepwise increase, which is an evidence of elastic-plastic stress wave propagation.

Figs. 2.19 (c) and (d) show the growths of modal amplitudes of the sandwich column with an imperfection magnitude $\zeta=0.05 \mathrm{c}$ calculated from the semi-analytical model. The lowest mode and the third one predominate for both velocities. In Fig. 2.19(e)-(h), the deformed shapes from the FE simulations and the semi-analytical model are illustrated near the point of failure. For $V=1 \mathrm{~m} / \mathrm{s}$ as shown in Fig. 2.19(e) and (g), the FE deformation shape indicates that the only dominant mode is the first eigenmode, $\mathrm{W}_{1}$ while the semi-analytical model shows the growth in the amplitude of the third eigenmode, $\mathrm{W}_{3}$. For $\mathrm{V}=5 \mathrm{~m} / \mathrm{s}$ (Fig. 2.19 (f) and (g)) the third eigenmode, $\mathrm{W}_{3}$, appears to be activated in the FE simulation, which is in good agreement with the analytical model. 


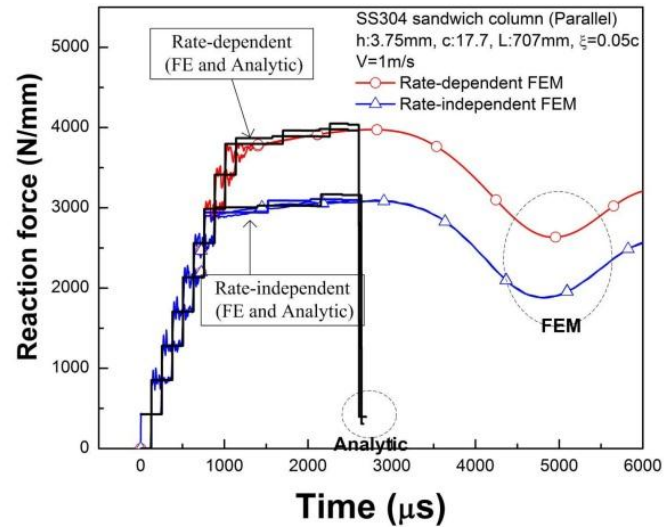

(a) $\mathrm{V}=1.0 \mathrm{~m} / \mathrm{s}$

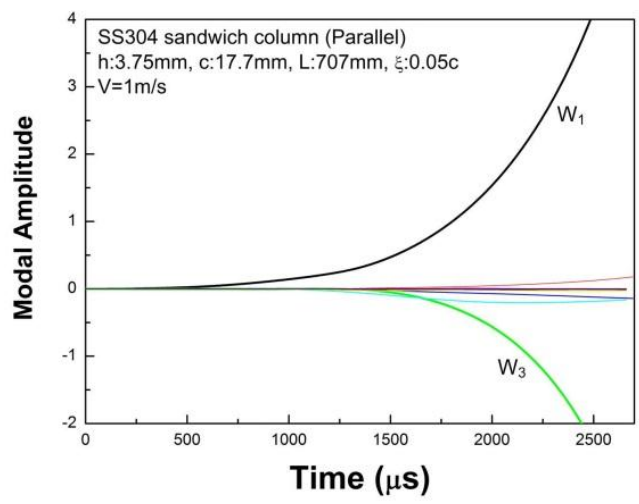

(c) $\mathrm{V}=1.0 \mathrm{~m} / \mathrm{s}$

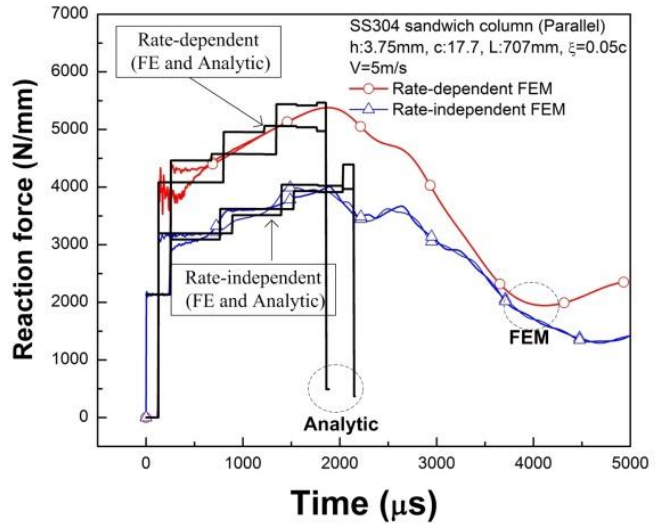

(b) $\mathrm{V}=5.0 \mathrm{~m} / \mathrm{s}$

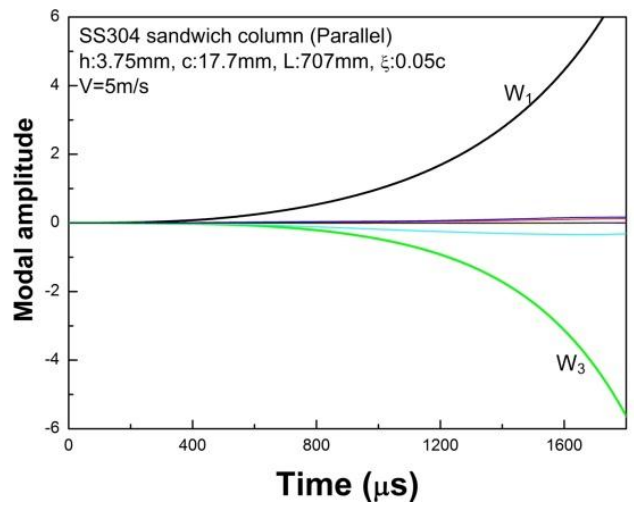

(d) $\mathrm{V}=5 \mathrm{~m} / \mathrm{s}$

(e) $\mathrm{V}=1.0 \mathrm{~m} / \mathrm{s}(\xi=0.05 \mathrm{c})$ at $\mathrm{t}=2667 \mu$ s obtained from FEM (Mag 10)

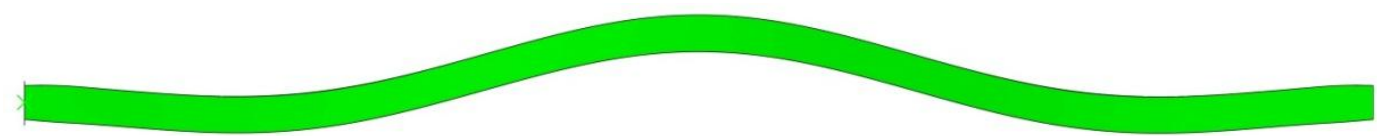

(f) $\mathrm{V}=5.0 \mathrm{~m} / \mathrm{s}(\xi=0.05 \mathrm{c})$ at $\mathrm{t}=2100 \mu$ s obtained from FEM (Mag 5)

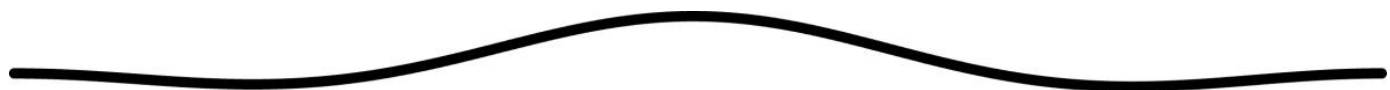

(g) $\mathrm{V}=1.0 \mathrm{~m} / \mathrm{s}(\xi=0.05 \mathrm{c})$ at $\mathrm{t}=2600 \mu \mathrm{s}$ obtained from the semi-analytical model

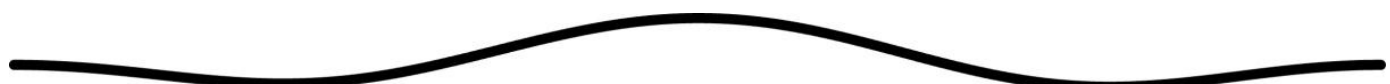

(h) $\mathrm{V}=5.0 \mathrm{~m} / \mathrm{s}(\xi=0.05 \mathrm{c})$ at $\mathrm{t}=1860 \mu \mathrm{s}$ obtained from the semi-analytical model

Figure 2.19: Dynamic response of an SS304 corrugated core sandwich column under compression velocities of $\mathrm{V}=1$ and $5 \mathrm{~m} / \mathrm{s}$ obtained from FEM and the semi-analytical $(\mathrm{h}=3.75 \mathrm{~mm}, \mathrm{t}=1.5 \mathrm{~mm}, \mathrm{l}=25.0 \mathrm{~mm}$, $\mathrm{w}=45^{\circ}, \bar{\rho}=12 \%, \mathrm{~L}=707 \mathrm{~mm}, \xi=0.05 \mathrm{c}$ ): (a)(b) Reaction force versus time history curves for $\mathrm{V}=1 \mathrm{~m} / \mathrm{s}$ and $5 \mathrm{~m} / \mathrm{s}$; (c)(d) The growth of modal amplitudes of the column $(\xi=0.05 \mathrm{c}$ ) for $\mathrm{V}=1 \mathrm{~m} / \mathrm{s}$ and $5 \mathrm{~m} / \mathrm{s}$; (e)(f) Deformed profiles of the column $(\xi=0.05 \mathrm{c})$ for $\mathrm{V}=1.0$ and $5.0 \mathrm{~m} / \mathrm{s}$ near the time-of-failure $(\mathrm{t}=2667$ and $2100 \mu \mathrm{s})$ obtained from the FE simulations (Mag 10); (g)(h) Deformed profiles of the column ( $\xi=0.05 \mathrm{c}$ ) for $\mathrm{V}=1$ and $5 \mathrm{~m} / \mathrm{s}$ the time-of-failure ( $\mathrm{t}=2600$ and $1860 \mu \mathrm{s})$ obtained from the semi-analytical model. 


\subsubsection{Effect of Core Shear Deformation}

The effect of core shear deformation on the global buckling motion of sandwich columns is investigated. It is generally known that core shear deformation effects are pronounced for columns of short column length and large cross-sectional area with low shear stiffness (e.g., sandwich columns) [45,46].

Accordingly, two SS304 corrugated core sandwich columns compressed parallelto-corrugations having different relative core densities (different core shear stiffness) are investigated: one is the SS304 corrugated core column compressed parallel-tocorrugations $\left(\mathrm{h}=3.75 \mathrm{~mm}, \mathrm{t}=1.5 \mathrm{~mm}, \mathrm{l}=25.0 \mathrm{~mm}, \mathrm{w}=45^{\circ}, \bar{\rho}=0.12, \mathrm{~L}=707 \mathrm{~mm}\right)$ presented previously, and the other column has all the same geometric dimensions but smaller core thickness $\left(\mathrm{h}=3.75 \mathrm{~mm}, \mathrm{t}=0.675 \mathrm{~mm}, \mathrm{l}=25.0 \mathrm{~mm}, \mathrm{w}=45^{\circ}, \bar{\rho}=0.0533, \mathrm{~L}=707 \mathrm{~mm}\right)$. Both columns are compressed at a velocity of $5 \mathrm{~m} / \mathrm{s}$ with an assigned imperfection magnitude of $\xi=0.05 \mathrm{c}$.

Fig. 2.20 shows the difference between the results calculated from the analytical models including and excluding core shear deformation. The analytical results without core shear deformation have been obtained by assuming that the shear rigidity, $\mathrm{S}$, is infinity in the model. For the sandwich column of $\bar{\rho}=0.12$, the core shear deformation effect is not substantial (4\% difference in times-to-failure) as shown in Fig. 2.20(a).

On the other hand, for the sandwich column having the more compliant core $(\bar{\rho}=0.0533)$, the difference between the two results (i.e., with and without considering core shear deformation) is more appreciable (9\% difference in times-to-failure) than that shown in the stiffer core sandwich column $(\bar{\rho}=0.12)$, but, not significant (less than $10 \%$ difference in times-to-failure) as well as shown in Fig. 2.20(b). 


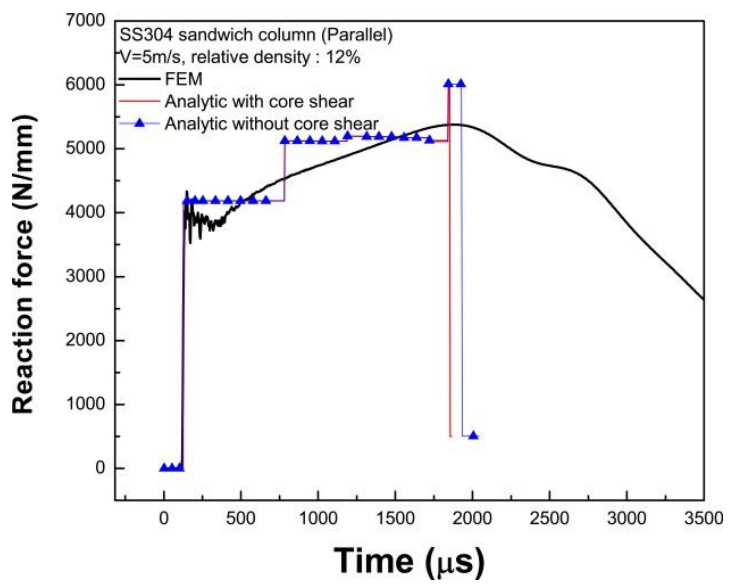

(a)

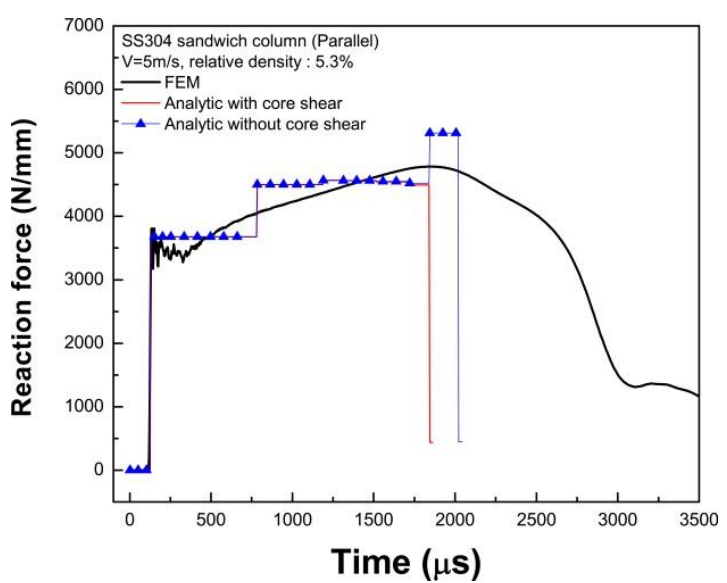

((b)

Figure 2.20: The effect of core shear deformation for SS304 corrugated core sandwich columns under $\mathrm{V}=5 \mathrm{~m} / \mathrm{s}$ of (a) $\mathrm{h}=3.75 \mathrm{~mm}, \mathrm{t}=1.5 \mathrm{~mm}, \mathrm{l}=25.0 \mathrm{~mm}, \mathrm{w}=45^{\circ}, \bar{\rho}=0.12, \mathrm{~L}=707 \mathrm{~mm}$; and (b) $\mathrm{h}=3.75 \mathrm{~mm}$, $\mathrm{t}=0.675 \mathrm{~mm}, \mathrm{l}=25.0 \mathrm{~mm}, \mathrm{w}=45^{\circ}, \bar{\rho}=0.0533, \mathrm{~L}=707 \mathrm{~mm}$.

Considering that core shear effect is more pronounced in the short-wave length deformation and weak-core sandwich columns, it did not change the time-to-failure dramatically for the considered range of relative density.

\subsection{CHAPTER SUMMARY}

In this chapter, semi-analytical models were developed to predict the dynamic global buckling failure of corrugated core sandwich columns until the time to maximum peak load, which is thought to be a point of loss of structural capability. The proposed semi-analytical models are in reasonable agreement with the FE results: the relative errors in peak reaction forces are within 10\% except for the A16061-T6 sandwich column compressed perpendicular-to-corrugations at $\mathrm{V}=0.5 \mathrm{~m} / \mathrm{s}$. It was successful in showing the imperfection-sensitive, velocity dependent dynamic response and appearance of higher buckling modes. In addition, strain-rate dependent response is successfully approximated in the semi-analytical models by treating the rate-dependent material as a rate- 
independent material with the adjusted material properties. Furthermore, the FE and semi-analytical results revealed the following.

1) As a loading rate increases, corrugated core sandwich columns compressed perpendicular-to-corrugations are more susceptible to face wrinkling.

2) The column that failed in the elastic region is very sensitive to magnitude of imperfections.

3) At a low loading rate, buckling modes are activated from the lowest buckling mode. However, at a higher loading rate at yielding, many buckling modes can be activated at the same time due to degraded plastic material properties.

4) Since the core in the perpendicular-to-corrugations direction makes little contribution to carrying axial loads, the corrugated core sandwich column in this direction has a lower load at yielding compared with that compressed parallel-tocorrugation.

5) The addition of a core shear deformation effect expedites the growth of global buckling motion leading to early failure, however, in the considered range of core relative density $(0.0533<\bar{\rho}<0.12)$, the effect was not shown to be significant (less than $10 \%$ difference in times-to-failure). Accordingly, the analysis neglecting the core shear deformation effect, presented in Section 2.4.1, can be used in the preliminary design stage to probe the dynamic response of corrugated core sandwich columns without sacrificing too much accuracy.

In conclusion, the semi-analytical model is worthwhile as a sandwich column design tool approximating the dynamic response for corrugated core sandwich columns of a variety of sandwich geometric dimensions under compression velocities if combined 
with an analytical model predicting the other failure mode, local buckling failure. 


\section{CHAPTER 3}

\section{AN ANALYTICAL MODEL FOR THE PREDICTION OF FACE WRINKLING}

\subsection{INTRODUCTION}

In this chapter, an analytical model is proposed for the prediction of face wrinkling behavior of corrugated core sandwich columns under dynamic compressive loading perpendicular-to-corrugations. The developed model accounts for complex dynamic phenomena due to the effects of material rate-dependence, inertia, and stress wave propagation.

The proposed analytical model is based on the calculation of transverse motion of face ligaments near the front and back ends of a sandwich column. The governing equation for the face ligaments is a dynamic version of Euler-Bernoulli beam-column equation [64], and the motions are obtained by employing the Galerkin method to solve the equation. The axial force-time history applied to a face ligament is estimated based on the theory of elastic and elastic-plastic wave propagation [70-73]. In addition, materialrate dependence is considered by treating a rate-dependent material as a rate-independent one with elevated yield strength and adjusted tangent modulus $[33,75,101]$.

This chapter is organized as follows: In Section 3.2, the dynamic problem of corrugated core sandwich columns compressed perpendicular-to-corrugations is established mathematically. In Section 3.3, the analytical model for the established 
problem is explained in detail. In Section 3.4, the model is validated through comparing several metrics such as reaction force and the deflection of face ligaments with $\mathrm{FE}$ simulations. The effects of rate-of-compression and imperfections are also investigated. Finally, the model's limitation and efficiency are discussed.

\subsection{PROBLEM STATEMENTS}

\subsubsection{Problem Definition}

In this chapter, the response of A16061-T6 and SS304 corrugated core sandwich columns dynamically loaded perpendicular-to-corrugations is studied (Fig. 3.1). One end (front end) is axially displaced at a constant rate of $\mathrm{V}$ with all other degrees of freedom (DOFs) constrained while a fixed end condition is imposed at the other end (back end).

The considered range of the compression rate is such that $V /\left(c_{e l}^{(\text {perp })} \varepsilon_{Y}\right)<1$ as in Chapter 2. Note that the apparent elastic stress wave speed, $\mathrm{c}_{e l}^{(\text {perp })}$, is related to both material properties and sandwich geometric parameters, as given in Eqn. (2.36). The maximum applied velocity satisfying the above condition can be of the order of a few $\mathrm{m} / \mathrm{s}$ for a typical design of corrugated core sandwich columns.

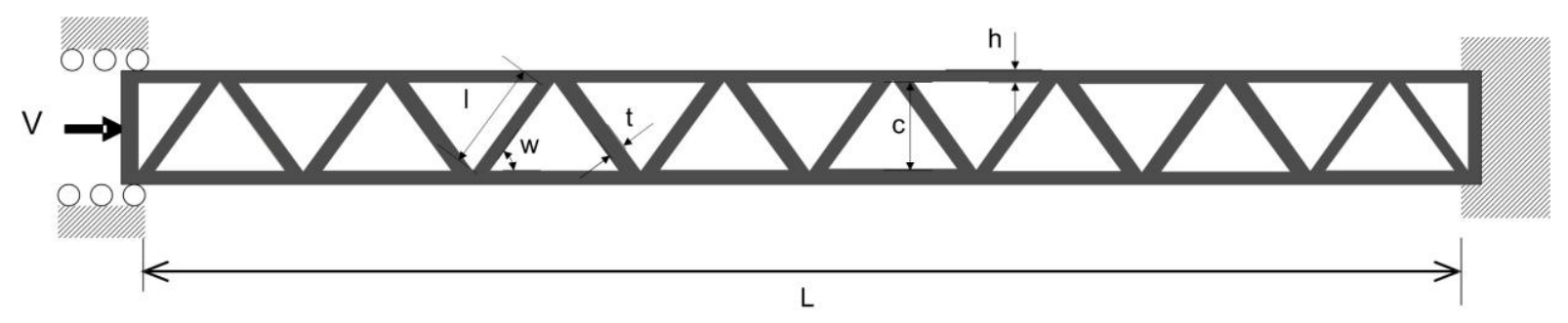

Figure 3.1: Dynamic problem of face wrinkling failure. 


\subsubsection{Mathematical Formulation}

The proposed analytical model for the dynamic face wrinkling problem considers the behavior of a face sheet as shown in Fig. 3.2. When one end of a corrugated core sandwich column is compressed in this loading configuration (perpendicular-tocorrugations) at a loading rate in the considered range, it is assumed that only the faces, not the corrugated core, resist the in-plane load because the deformation of the core is mainly due to folding at nodes.

A face sheet ligament of thickness, $\mathrm{h}$, and span length, $2 \ell \cdot \cos \omega$, is modeled as an Euler-Bernoulli beam-column subject to a time-varying axial force, $P(t) / 2$. The governing equation of motion based on the classical Euler-Bernoulli beam theory for the face sheet ligament can be given by

$$
D^{(f)} \frac{\partial^{4} w^{(f)}}{\partial x^{4}}+\frac{\partial}{\partial x}\left(\frac{P(t)}{2} \frac{\partial w^{(f)}}{\partial x}\right)+\rho h \frac{\partial^{2} w^{(f)}}{\partial t^{2}}=-\frac{\partial}{\partial x}\left(\frac{P(t)}{2} \frac{\partial w_{0}^{(f)}}{\partial x}\right)
$$

The initial conditions and boundary conditions for the governing equation (3.1) are given as below:

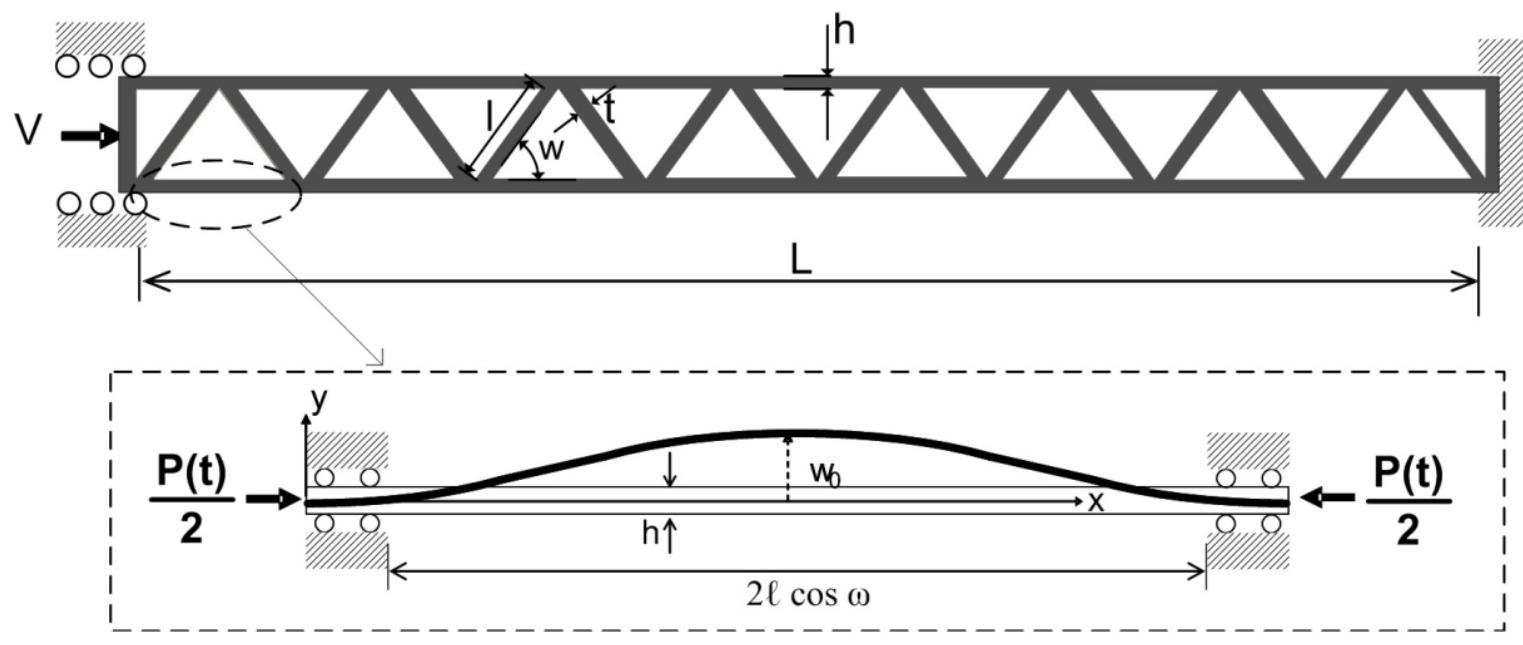

Figure 3.2: Euler beam-column modeling of a face ligament for the dynamic face wrinkling. 


$$
\begin{aligned}
& w^{(f)}(x, 0)=a(x) \\
& \frac{\partial w^{(f)}(x, 0)}{\partial t}=b(x) \\
& w^{(f)}(0, t)=0 \\
& \frac{\partial w^{(f)}(0, t)}{\partial x}=0 \\
& w^{(f)}(2 \ell \cos \varpi, t)=0 \\
& \frac{\partial w^{(f)}(2 \ell \cos \varpi, t)}{\partial x}=0
\end{aligned}
$$

The governing equation of motion (3.1) is given in terms of the flexural rigidity of a face sheet ligament, $\mathrm{D}^{(\mathrm{f})}$, initial imperfections, $\mathrm{w}_{0}^{(f)}$, axial force, $P(t) / 2$, and density, $\rho$. The flexural rigidity, $\mathrm{D}^{(\mathrm{f})}$, is given by

$$
\begin{aligned}
& D^{(f)}=\frac{E h^{3}}{12\left(1-v^{2}\right)} \text { if the face material is elastic, } \\
& D^{(f)}=\frac{E_{T}^{\prime} h^{3}}{12} \text { if the face material is plastic. }
\end{aligned}
$$

If an elastic column is compressed at a rate small enough such that axial inertia can be ignored, the function, $P(t) / 2$, can be simply assumed to be linear with time. However, if the axial inertia effect cannot be neglected, the linear function approximation of $P(t) / 2$ is not valid any more [39]. Instead, axial stress wave propagation effects should be taken into account in that regard. 


\subsection{ANALYTICAL MODEL}

The following assumptions are made in the development of the face wrinkling analytical model.

(1) The contribution of a corrugated core to the in-plane stiffness of a sandwich column is negligible compared to that of face sheets when the sandwich column is compressed perpendicular-to-the-corrugations.

(2) The stress wave propagation along sandwich column length, L, might be important. However, the axial stress distribution in a face sheet ligament is considered to be spatially uniform. This assumption is based on the fact that

$$
\frac{2 \ell \cos \varpi}{c_{e l}^{(m)}} \ll \frac{L}{c_{e l}^{(P e r p)}}
$$

Consequently, the axial force within a face sheet ligament is a function of time only, $\mathrm{P}(\mathrm{t}) / 2$.

(3) The axial force applied to a face ligament, $\mathrm{P}(\mathrm{t}) / 2$, increases stepwise with respect to time, $t$, and can be estimated by adopting the theory of elastic and elastic-plastic wave propagation [70-73].

(4) The face wrinkling mode is assumed to be decoupled with another deformation mode, global buckling motion. That is, no interaction is assumed, especially when estimating $\mathrm{P}(\mathrm{t})$.

(5) All of the face ligaments have the same dynamic buckling strength and imperfections. Based on this assumption, one of the face ligaments close to both the ends are the most susceptible to face wrinkling. (i.e., one of the face ligaments close to both the ends is subjected to compressive loading, either 
with greater magnitude or for longer duration, than any other face ligaments in the sandwich column)

The face wrinkling motion of the two face ligaments at the both ends (back and front ends) are obtained by solving the analytical model to be explained in the following subsections, 3.3.1 through 3.3.5.

\subsubsection{Estimation of Axial Force History}

Based on the basic idea explained in the previous section, Section 2.3, the axial forces, $P(t) / 2$, that develop in the face ligaments closest to the both ends can be estimated individually. The estimation of the axial force history for corrugated core sandwich columns starts from the analogy between stress wave propagations in a monolithic solid column and a corrugated core sandwich column.

Consider the face ligament at the front end indicated by the red dashed circle in Fig. 3.3(a). When one end is suddenly set in motion at a constant rate of $\mathrm{V}$, the face ligament is subject to an axial force, $\frac{P(t)}{2}=\mathrm{h} \cdot \Delta \sigma_{e l}^{(P e r p)}$ during one round trip of the elastic stress wave front, $2 \Delta t_{e l}^{(\text {Perp })}$ (i.e., $\left.0<\mathrm{t}<2 L / c_{e l}^{(\text {Perp })}\right) . \Delta \sigma_{e l}^{(\text {Perp })}$ and $c_{e l}^{(\text {Perp })}$ are given in Section 2.3.2.

$$
\begin{gathered}
c_{e l}^{(\text {perp })}=\sqrt{\frac{E}{\left(1-v^{2}\right) \frac{2 h}{(2 h+\bar{\rho} c) \rho}}} \\
\Delta \sigma_{e l}^{(p e r p)}=\sqrt{\frac{2 h E(2 h+\bar{\rho} c) \rho}{1-v^{2}}} V
\end{gathered}
$$




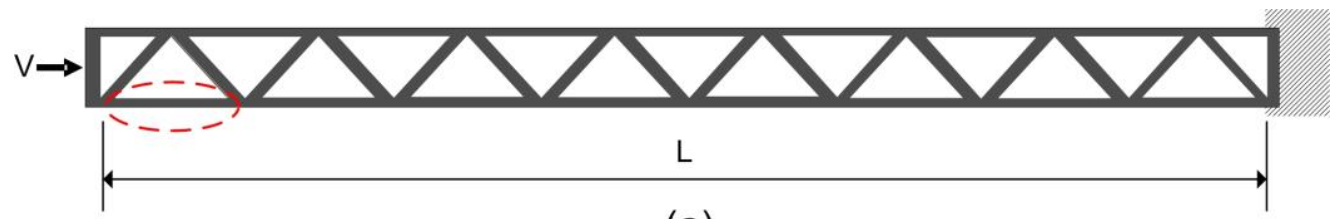

(a)

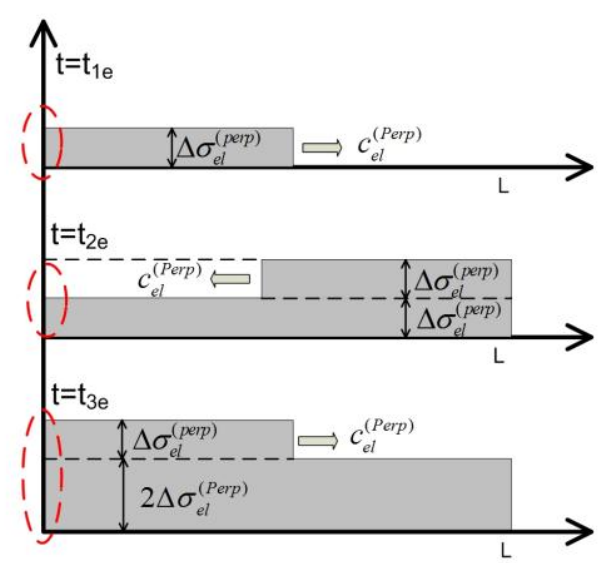

(b)

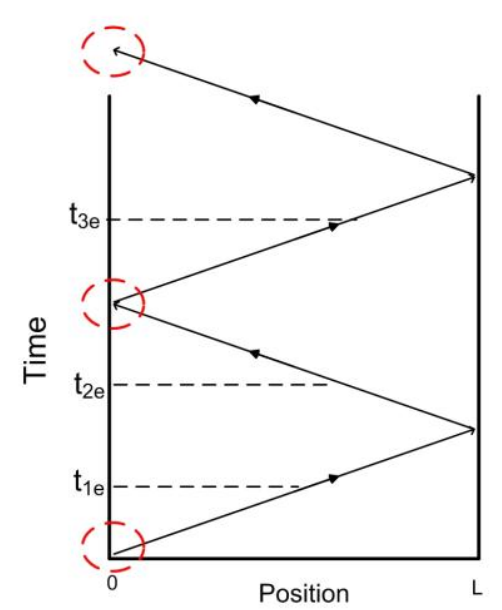

(c)

Figure 3.3: Estimation of axial force history of face ligaments during elastic stress wave propagation: (a) the target face ligament at the front end (red dash circle); (b) axial stress distribution at $t=t_{1 e}, t_{2 e}, t_{3 e}$; (c) Largange $\mathrm{x}$-t diagram indicating the position of wave fronts.

After the second elastic stress wave reflection $\left(\mathrm{t}=2 L / c_{e l}^{(\text {Perp })}\right)$, the face ligament at the front end is under $\frac{P(t)}{2}=\mathrm{h} \cdot\left(3 \Delta \sigma_{e l}^{(P e r p)}\right)$ for another time period of $2 \Delta t_{e l}^{(P e r p)}=$ $2 L / c_{e l}^{(\text {Perp })}$. Therefore, the face ligament at the front end during elastic stress wave propagation can be summarized as follows.

i) Axial force history of the front end face ligament during elastic stress wave propagation.

$$
\begin{gathered}
\frac{P(t)}{2}=h \cdot\left(\Delta \sigma_{e l}^{(\text {Perp })}\right) \quad \text { for } 0<t<2 \Delta t_{e l}^{(\text {Perp })} \\
\frac{P(t)}{2}=h \cdot\left(3 \Delta \sigma_{e l}^{(\text {Perp })}\right) \quad \text { for } 2 \Delta t_{e l}^{(\text {Perp })}<t<4 \Delta t_{e l}^{(\text {Perp })} \\
\vdots \\
\frac{P(t)}{2}=h \cdot\left((2 k-1) \Delta \sigma_{e l}^{(\text {Perp })}\right) \quad \text { for } 2(k-1) \Delta t_{e l}^{(\text {Perp })}<t<2 k \Delta t_{e l}^{(\text {Perp })}
\end{gathered}
$$


Moreover, the axial force-time history in the back end face ligament during elastic stress wave propagaion can be deduced in a similar manner. Refer to column stress distribution and Lagrange $\mathrm{x}$-t diagram indicating the propagating wave front location in Figs. 3.3(b) and (c), respectively.

ii) Axial force history of the back end face ligament during elastic stress wave propagation

$$
\begin{gathered}
\frac{P(t)}{2}=h \cdot\left(2 \Delta \sigma_{e l}^{(\text {Perp })}\right) \quad \text { for } \quad \Delta t_{e l}^{(\text {Perp })}<t<3 \Delta t_{e l}^{(\text {Perp })} \\
\frac{P(t)}{2}=h \cdot\left(4 \Delta \sigma_{e l}^{(\text {Perp })}\right) \quad \text { for } 3 \Delta t_{e l}^{(\text {Perp })}<t<5 \Delta t_{e l}^{(\text {Perp })} \\
\vdots \\
\frac{P(t)}{2}=h \cdot\left((2 k) \Delta \sigma_{e l}^{(\text {Perp })}\right) \quad \text { for } \quad(2 k-1) \Delta t_{e l}^{(\text {Perp })}<t<(2 k+1) \Delta t_{e l}^{(\text {Perp })}
\end{gathered}
$$

Suppose that elastic-plastic transition occurs at the back end at time $t=t$ ' between $t_{1 P}$ and $\mathrm{t}_{2 \mathrm{P}}$ as shown in Fig. 3.4. At $\mathrm{t}=\mathrm{t}^{\prime}$, an elastic wave front of $\Delta \sigma_{1}^{(\text {Perp })}=\sigma_{Y}^{(\text {Perp })}-$ $\sigma_{1}^{(\text {Perp })}$ and a plastic wave front of $\Delta \sigma_{2}^{(\text {Perp })}=\sqrt{\frac{E_{T}^{\prime}}{E /\left(1-v^{2}\right)}}\left(\Delta \sigma_{e l}^{(\text {Perp })}-\Delta \sigma_{1}^{(\text {Perp })}\right)$ propagate with wave speeds of $c_{e l}^{(\text {Perp })}$ and $c_{p l}^{(\text {Perp })}$ toward the other end. Here, $\sigma_{1}^{(\text {Perp })}$ denotes the axial stress just before $t=t^{\prime}$. The elastic stress wave front reaches the other end (red dash circle in the illustration of Fig. 3.4(a)). Reflection of the elastic wave front at the other end occurs at $\mathrm{t}=\mathrm{t}^{\prime}+\Delta t_{e l}^{(\text {Perp })}$. Consequently, two plastic wave fronts with magnitudes of $\Delta \sigma_{1}^{(\text {Perp })}$, and $\Delta \sigma_{2}^{(\text {Perp })}$ travel independently. Here, $\Delta \sigma_{1}^{(\text {Perp })^{\prime}}=$ $\sqrt{\frac{E_{T}^{\prime}}{E /\left(1-v^{2}\right)}} \cdot \Delta \sigma_{1}^{(\text {Perp })}$, and $c_{p l}^{(\text {Perp })}, \sigma_{Y}^{(\text {Perp })}$ are given in Chapter 2. 


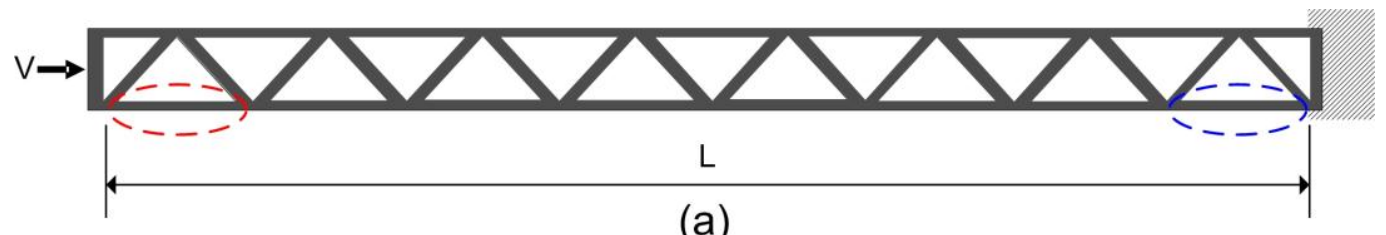

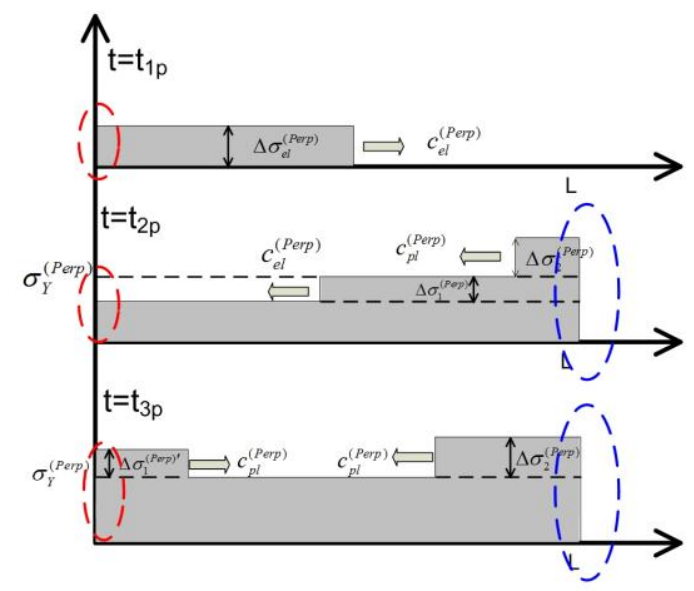

(b)

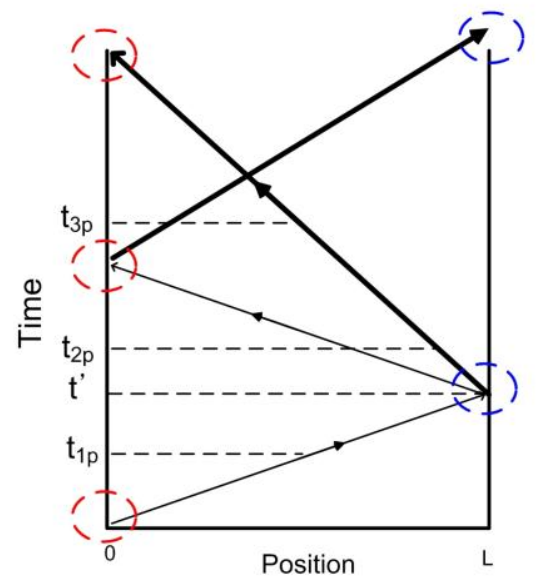

(c)

Figure 3.4: Estimation of axial force history of face ligaments during elastic-plastic stress wave propagation: (a) Two face ligaments at front and back ends (elastic-plastic transition occurs at the end near the face ligament in blue dash circle); (b) axial stress distribution at $t=t_{1 p}, t_{2 p}, t_{3 p}$; (c) Largange $x-t$ diagram indicating the positions of wave fronts.

Considering the stress distribution with respect to time and the Lagrange $\mathrm{x}-\mathrm{t}$ diagram in Figs. 3.4(b) and (c), $\frac{P(t)}{2}$ at the end where elastic-plastic transition occurs can be estimated as follows.

iii) Axial force history of a face ligament near the end where elastic-plastic transition occurs (it can be either the front end or the back end.)

$$
\begin{aligned}
& \frac{P(t)}{2}=h \cdot\left[\sigma_{Y}^{(\text {Perp })}+\left(\Delta \sigma_{2}^{(\text {Perp })}\right)\right] \quad \text { for } \quad t^{\prime}<t<t^{\prime}+\Delta t_{e l}^{(\text {Perp })}+\Delta t_{p l}^{(\text {Perp })} \\
& \frac{P(t)}{2}=h \cdot\left[\sigma_{Y}^{(\text {Perp })}+\left(\Delta \sigma_{2}^{(\text {Perp })}\right)+2\left(\Delta \sigma_{1}^{(\text {Perp })}\right)\right] \quad \text { for } \quad t^{\prime}+\Delta t_{e l}^{(\text {Perp })}+\Delta t_{p l}^{(\text {Perp })}<t<t^{\prime}+2 \Delta t_{p l}^{(\text {Perp })} \\
& \frac{P(t)}{2}=h \cdot\left[\sigma_{Y}^{(\text {Perp })}+3\left(\Delta \sigma_{2}^{(\text {Perp })}\right)+2\left(\Delta \sigma_{1}^{(\text {Perp })}\right)\right] \quad \text { for } \quad t^{\prime}+2 \Delta t_{p l}^{(\text {Perp })}<t<t^{\prime}+\Delta t_{\text {el }}^{(\text {Perp })}+3 \Delta t_{p l}^{(\text {Perp })} \\
& \frac{P(t)}{2}=h \cdot\left[\sigma_{Y}^{(\text {Perp })}+3\left(\Delta \sigma_{2}^{(\text {Perp })}\right)+4\left(\Delta \sigma_{1}^{(\text {Perp })}\right)\right] \quad \text { for } \quad t^{\prime}+\Delta t_{e l}^{(\text {Perp })}+3 \Delta t_{p l}^{(\text {Perp })}<t<t^{\prime}+4 \Delta t_{p l}^{(\text {Perp })}
\end{aligned}
$$


iv) Axial force history of a face ligament near the end other than the one stated in iii).

$$
\begin{aligned}
& \frac{P(t)}{2}=h \cdot\left[\sigma_{1}^{(\text {Perp })}\right] \quad \text { for } t^{\prime}<t<t^{\prime}+\Delta t_{e l}^{(\text {Perp })} \\
& \frac{P(t)}{2}=h \cdot\left[\sigma_{Y}^{(\text {Perp })}+\left(\Delta \sigma_{1}^{(\text {Perp })}\right)\right] \quad \text { for } \quad t^{\prime}+\Delta t_{e l}^{(\text {Perp })}<t<t^{\prime}+\Delta t_{p l}^{(\text {Perp })} \\
& \frac{P(t)}{2}=h \cdot\left[\sigma_{Y}^{(\text {Perp })}+2\left(\Delta \sigma_{2}^{(\text {Perp })}\right)+\left(\Delta \sigma_{1}^{(\text {Perp })}\right)\right] \quad \text { for } \quad t^{\prime}+\Delta t_{p l}^{(\text {Perp })}<t<t^{\prime}+\Delta t_{e l}^{(\text {Perp })}+2 \Delta t_{p l}^{(\text {Perp })} \\
& \frac{P(t)}{2}=h \cdot\left[\sigma_{Y}^{(\text {Perp })}+2\left(\Delta \sigma_{2}^{(\text {Perp })}\right)+3\left(\Delta \sigma_{1}^{(\text {Perp })}\right)\right] \quad \text { for } \quad t^{\prime}+\Delta t_{e l}^{(\text {Perp })}+2 \Delta t_{p l}^{(\text {Perp })}<t<t^{\prime}+3 \Delta t_{p l}^{(\text {Perp })}
\end{aligned}
$$

In short, the axial-force history of selected face ligaments can be estimated by the procedure in this section.

\subsubsection{Galerkin Method for Face Ligaments with Initial Curvatures}

The transverse motions, $\mathrm{w}^{(\mathrm{f})}(\mathrm{x}, \mathrm{t})$, of the face sheet ligaments near the front and back ends are calculated by solving the governing equation of motion with the estimated axial force, $\frac{P(t)}{2}$, using the Galerkin method.

$$
\int_{0}^{2 l \cos w}\left[D^{(f)} \frac{\partial^{4} w^{(f)}}{\partial x^{4}}+\frac{\partial}{\partial x}\left(\frac{P(t)}{2} \frac{\partial\left(w^{(f)}+w_{0}^{(f)}\right)}{\partial x}\right)+\rho h \frac{\partial^{2} w^{(f)}}{\partial t^{2}}\right] \cdot \delta w^{(f)}=0
$$

The transverse motion, $\mathrm{w}^{(f)}(x, t)$, initial curvature imperfection, $w_{0}^{(f)}(x)$, and virtual displacement, $\delta \mathrm{w}^{(f)}$, are given in the form of an eigenfunction expansion, a combination of the first two eigenmodes, $W_{1}^{(f)}(x)$ and $W_{2}^{(f)}(x)$, from a static buckling analysis.

$$
\begin{gathered}
w^{(f)}(x, t)=\sum_{i=1}^{2} T_{i}^{(f)}(t) \cdot W_{i}^{(f)}(x) \\
w_{0}^{(f)}(x)=\sum_{i=1}^{2} \xi_{i}^{(f)} \cdot W_{i}^{(f)}(x)
\end{gathered}
$$




$$
\delta w^{(f)}=\sum_{i=1}^{2} \delta T_{i}^{(f)} \cdot W_{i}^{(f)}(x)
$$

The two eigenfunctions are:

$$
\begin{gathered}
W_{1}^{(f)}(x)=\frac{1}{2}\left(1-\cos \frac{2 \pi x}{2 \ell \cos \varpi}\right) \\
W_{2}^{(f)}(x)=A\left(\sin k x-k x-\frac{k(2 \ell \cos \varpi)}{2} \cos k x+\frac{k(2 \ell \cos \varpi)}{2}\right)
\end{gathered}
$$

where $k=8.987 / 2 \ell \cos \varpi$, and $\mathrm{A}$ is a normalizing factor.

The integral expression written in Eqn. (3.10) is transformed into ordinary differential equations (ODEs) with respect to $T_{1}^{(f)}(t)$ and $T_{2}^{(f)}(t)$. Finally, the ODEs are solved using a $4^{\text {th }}$ order Runge-Kutta method until the failure criterion, described in the next subsection is satisfied.

\subsubsection{Failure Criterion}

Failure is defined as the time when a sudden load drop in the reaction force history curve occurs, which is equivalent to the time at peak reaction force. When this type of instability in a compressive member occurs, excessive deformation together with a high deformation rate results in a sudden loss of load-carrying capacity. To account for this phenomenon, a load drop criterion is defined such that a significant load drop is assumed if the extension rate of the mid-plane of face sheet ligament exceeds the compressive rate [74].

$$
\frac{d \Delta l_{e x t}^{(f)}}{d t} \geq \frac{d \Delta l_{c o m p}^{(f)}}{d t}
$$


where

$$
\frac{d \Delta l_{e x t}^{(f)}}{d t} \approx \int_{0}^{2 l \cos \omega}\left[\frac{\partial}{\partial t}\left(\frac{\partial w^{(f)}}{\partial x}\right)\right]\left(\frac{\partial w^{(f)}}{\partial x}\right) d x+\int_{0}^{2 l \cos \omega}\left[\frac{\partial}{\partial t}\left(\frac{\partial w^{(f)}}{\partial x}\right)\right]\left(\frac{d w_{0}^{(f)}}{d x}\right) d x
$$

$$
\frac{d \Delta l_{c o m p}^{(f)}}{d t} \approx V \cdot \frac{2 l \cos w}{L}
$$

\subsubsection{Inclusion of Material Rate-Dependence}

The model explained so far pertains to a column composed of a rate-independent material. To include material rate-dependence, the analytical model considers the rate dependent material as a rate-independent material with elevated yield strength and adjusted strain-hardening modulus as done in Chapter 2.

$$
\begin{gathered}
\sigma_{Y}^{(m)}=\frac{R \cdot \sigma_{y}^{0}}{\sqrt{1-v_{P}+v_{P}^{2}}} \\
E_{T}^{\prime(m)}=\frac{1}{1 /(E)-v /(2 E)+3 /\left(4 R E_{p}\right)}
\end{gathered}
$$

For the dynamic response of rate-dependent sandwich columns, the face wrinkling analytical procedure is repeated for the rate-independent material with adjusted material properties.

\subsubsection{Consideration of Sandwich Columns having Perfect Geometry}

In order to make a transverse motion in compressive members, a moment initiator such as geometric curvature imperfections or load eccentricity is required for numerical implementation. It is usual practice to assign geometric curvature imperfections in FE simulations $[3-5,29,48,51]$. Sometimes, a round-off error can be one source leading to such motion as an asymptotic value $[3,102]$ 
With regard to moment initiating sources, the corrugated core in the direction of perpendicular-to-corrugations appears to have special structural characteristics: When a corrugated core sandwich column is compressed, the main deformation mechanism of the corrugated core is folding of corrugations at nodes rather than stretching or flexural deformations of corrugation legs. Thus, the vertical position change of a node between a face and a core can initiate a motion of a face ligament causing face wrinkling failure in the sandwich columns of perfect geometry as illustrated in Fig. 3.5. Therefore, the analysis considering this Poisson effect may result in an asymptotic dynamic strength of sandwich columns having no imperfection.

In the analytical model, face wrinkling due to the Poisson effect is realized by assigning initial conditions (i.e., the amount of vertical position change of a node during the first round trip of an incident elastic stress wave is used as an approximating function of $a(x)$ in Eqn. (3.2a)).

$$
\begin{aligned}
& a(x) \approx a_{1} \cdot W_{1}^{(f)}(x)+a_{2} \cdot W_{2}^{(f)}(x) \\
& \text { where } a_{1}=\frac{1}{2} \cdot\left(\frac{\Delta \sigma_{e l}^{(\text {Perp })}}{E /\left(1-v^{2}\right)} \ell \cos (\omega)\right) \tan \left(\frac{\pi}{2}-\omega\right) \\
& a_{2}=a_{1} / 5
\end{aligned}
$$

The inclusion of the term, $\mathrm{a}_{2} \cdot W_{2}^{(f)}(x)$, is for convergence check in order to confirm that the second mode does not grow, leading to failure. The procedure for obtaining $\mathrm{a}_{1}$ in Eqn. (3.16) is given in Appendix B.

Therefore, the dynamic face wrinkling problems for sandwich columns with perfect geometry are solved by the analytical model using Eqn. (3.10) through Eqn. (3.15) with $\mathrm{a}(\mathrm{x})$ written in Eqn. (3.16). 


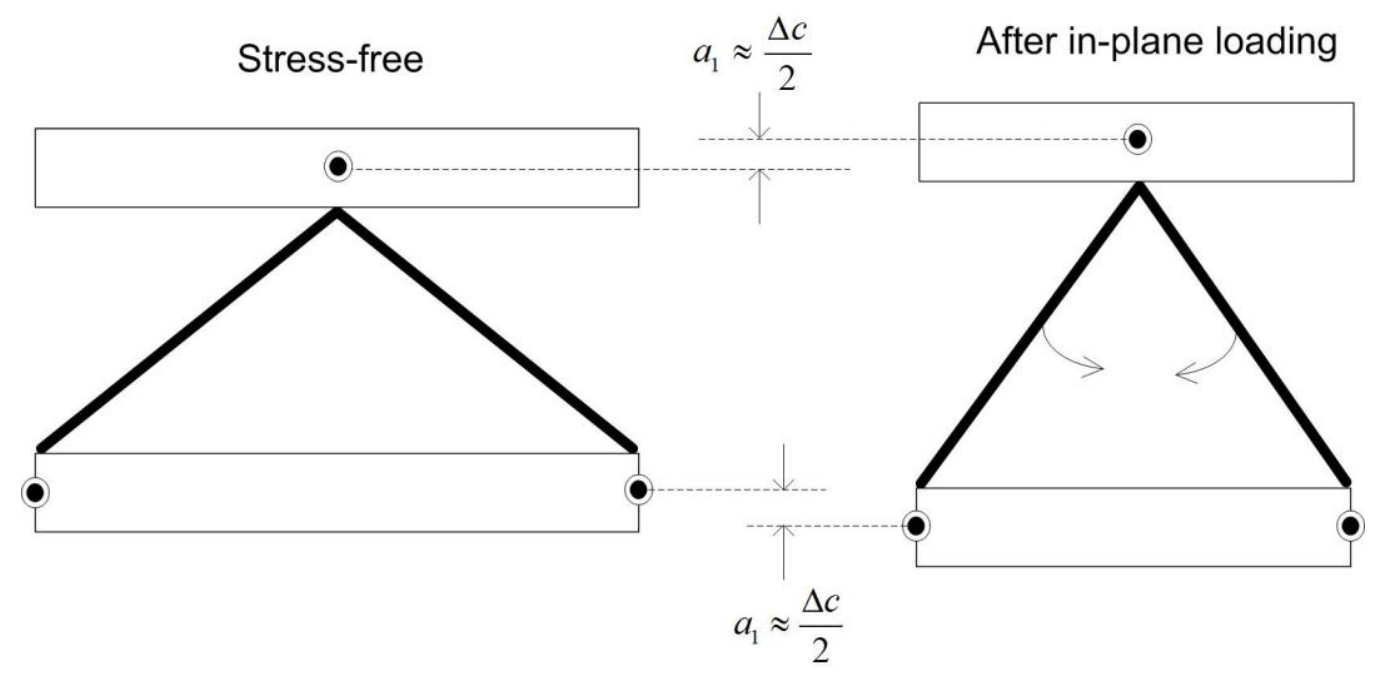

Figure 3.5: Change of vertical position of a node due to in-plane loading.

\subsubsection{Validation with Finite Element Simulations}

FE simulations are performed to validate the proposed analytical model. Comparison metrics for the validation are peak reaction forces at front and back ends as a function of imperfection magnitude and applied velocities, and transverse deflection at the midpoint of a face ligament. In addition, the underlying assumption regarding the analogy between stress wave propagations of monolithic and sandwich columns is also verified through comparison of the apparent elastic stress wave propagation speed, $c_{e l}^{(p e r p)}$, by FE measurements and Eqn. (2.36).

The FE models are constructed as discussed in Chapter 2.5, and the details are as such. Most of the validations are done on sandwich columns of perfect geometry except for the investigation of imperfection-sensitivity response. 


\subsection{RESULTS AND DISCUSSION}

The mathematical expression, Eqn. (2.36), for the apparent elastic stress wave speed, $c_{e l}^{(P e r p)}$, for sandwich columns is verified through FE simulations. This is followed by validation of the face wrinkling analytical model. It is an important derivation to solve the governing equation (3.10) because the analytical model assumes the analogy between stress wave propagations in monolithic solid columns and corrugated core sandwich columns. Finally, the FE simulations are compared with the analytical model for corrugated core sandwich columns compressed perpendicular-to-corrugations of various geometric dimensions under several applied velocities.

\subsubsection{Verification of Apparent Elastic Stress Wave Speed}

The apparent elastic stress wave speed ${ }^{1}, c_{e l}^{(\text {Perp })}$, is obtained from FE calculations by measuring the time period for several round-trips of an elastic stress wave along column length, L. The FE measurement is possible in that a stepwise increase in the reaction force curve is observed in axial compression of a straight column unless a significant transverse deflection of the sandwich column occurs.

The FE study has been done on two types of corrugated core sandwich columns with various face sheet thicknesses, h: 1) Al6061-T6 corrugated core sandwich columns with $\mathrm{L}=1877 \mathrm{~mm}$ and $\bar{\rho}=25 \%\left(\mathrm{t}=3.175 \mathrm{~mm}, \mathrm{l}=22 \mathrm{~mm}, \mathrm{w}=60^{\circ}\right)$ and 2$) \mathrm{SS} 304$ sandwich

1 The word "apparent" is added after confirming FE simulations on the axial wave characteristics of sandwich columns. In microscopic time scale during which an axial wave propagates one unit cell or so, the axial wave front in sandwich columns does not look planar in the $y-z$ plane due to the effect of the core (rather, zig-zag in top and bottom faces). On the other hand, the reaction force increase at one end is achieved in stepwise pattern macroscopically. 


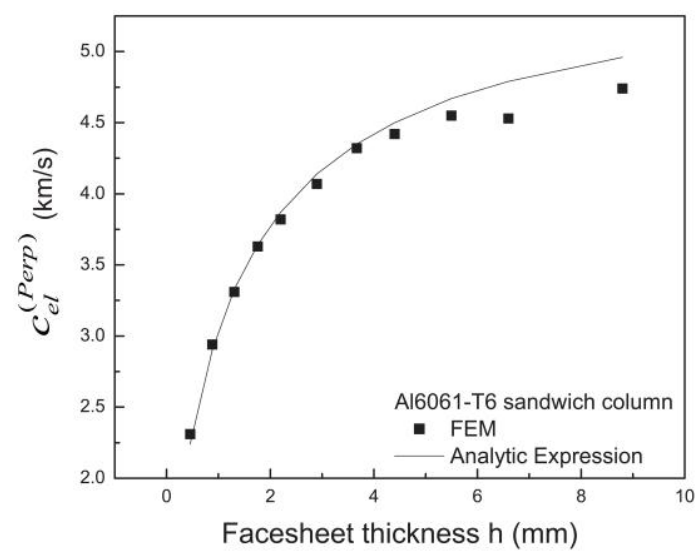

(a)

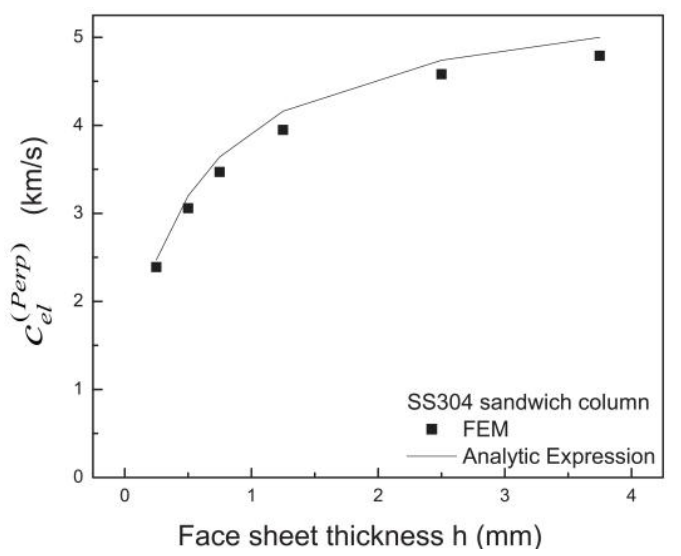

(b)

Figure 3.6: Apparent elastic stress wave speed $c_{e l}^{(\text {Perp })}$ of (a) Al6061-T6 sandwich columns (L=1877mm, $\bar{\rho}=25 \%)$ and (b) SS304 sandwich columns ( $\mathrm{L}=2828 \mathrm{~mm}, \bar{\rho}=12 \%)$ of varied face sheet thickness, h.

columns with $\mathrm{L}=2828 \mathrm{~mm}$ and $\bar{\rho}=12 \%\left(\mathrm{t}=1.5 \mathrm{~mm}, \mathrm{l}=25 \mathrm{~mm}, \mathrm{w}=45^{\circ}\right.$. On those two types of columns specified above, an end compression with low constant velocities $(\mathrm{V}=0.1 \mathrm{~m} / \mathrm{s}$ for most cases except for $\mathrm{V}=0.01 \mathrm{~m} / \mathrm{s}$ for $\mathrm{SS} 304$ columns of $\mathrm{h}=0.25,0.5 \mathrm{~mm}$ ) is applied so as to minimize the global column deflection.

Fig. 3.6 shows the apparent elastic stress wave speed, $c_{e l}^{(\text {Perp })}$, obtained from Eqn. (2.36) and the FE calculations for varying face sheet thickness $h$ for the specified $L$ and $\bar{\rho}$. The analytic expression of $c_{e l}^{(P e r p)}$, Eqn. (2.36), is in good agreement with the FE measurements. As a result, the underlying assumption is proved to be valid. It is

noteworthy that the analytic expression of $c_{e l}^{(P e r p)}$ in Eqn. (2.36) is also a function of sandwich design parameters, $h$ and $\bar{\rho}$ as well as material properties while the wave speed of monolithic columns is solely dependent on their material properties.

\subsubsection{Validation of the Analytical Model}

Validation of the analytical model is done on a variety of Al6061-T6 and SS304 
corrugated core sandwich columns of geometries under varied applied velocities. The geometric dimensions are classified into EFW and PFW, which are selected such that each sandwich column failed in either elastic face wrinkling (EFW) or plastic face wrinkling (PFW) under quasi-static loading, respectively. The geometric dimensions of the representative sandwich columns are listed in Table 3.1. In addition to the representative column geometries, a longer column — achieved by increasing the number of unit cells of a representative column (SS304-EFW) — is also examined to investigate the effect of overall column geometry.

Figures 3.7(a) and (b) show the reaction force versus time history curves of SS304 sandwich columns of the EFW and PFW geometric dimensions under $V=0.1 \mathrm{~m} / \mathrm{s}$ and $\mathrm{V}=1 \mathrm{~m} / \mathrm{s}$, respectively. The curves from FE simulations and the analytical model are based on the perfect geometry solution presented in Section 3.3.5. Arrows in the figures indicate the quasi-static strength of the sandwich columns, $P_{c r}^{(Q S)}$, which is equivalent to the quasi-static strength of a face ligament using Euler buckling formula for the EFW column or using the tangent modulus theory of Shanley for the PFW one, respectively [64]. It is revealed from both the FE simulations and analytical models that dynamic strengths are enhanced compared to the quasi-static strengths. In Figures 3.7(c) and (d),

Table 3.1. The geometric dimensions of the representative columns.

\begin{tabular}{cccccccc}
\hline Type of sandwich columns & $\begin{array}{c}\mathrm{h} \\
(\mathrm{mm})\end{array}$ & $\begin{array}{c}\mathrm{t} \\
(\mathrm{mm})\end{array}$ & $\begin{array}{c}\mathrm{1} \\
(\mathrm{mm})\end{array}$ & $\begin{array}{c}\mathrm{W} \\
\left({ }^{\circ}\right)\end{array}$ & $\begin{array}{c}\overline{\mathrm{L}} \\
(\%)\end{array}$ & $\begin{array}{c}\mathrm{P}_{c r}^{(Q S)} \\
(\mathrm{mm})\end{array}$ & $\begin{array}{c}\mathrm{N} / \mathrm{mm}) \\
\text { SS304 (EFW) }\end{array}$ \\
\hline \hline SS304 (PFW) & 0.25 & 1.5 & 25 & 45 & 12 & 353 & 21.1 \\
Al6061-T6 (EFW) & 3.75 & 1.5 & 25 & 45 & 12 & 353 & 2889.3 \\
Al6061-T6 (PFW) & 0.457 & 3.175 & 22 & 60 & 25 & 234 & 103.9 \\
\hline
\end{tabular}


the deformation shapes obtained from the FE calculations are exhibited before and after the maximum peak loads of the representative SS304 sandwich columns. Likewise, the analytical results for the A16061-T6 representative sandwich columns of the EFW and the PFW geometric dimensions are compared to FE calculations in Fig. 3.8.

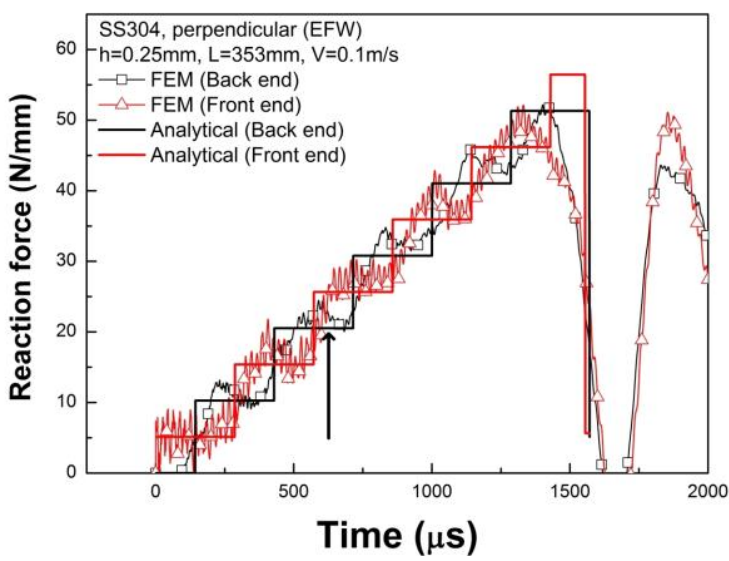

(a) $\mathrm{EFW}$

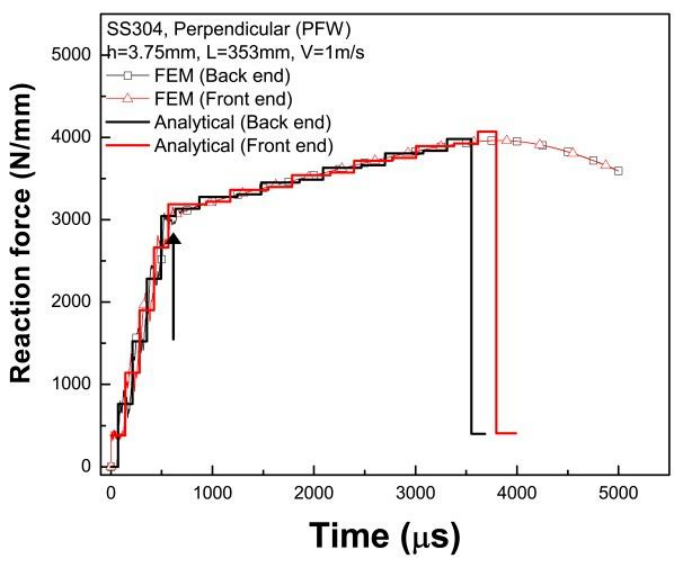

(b) PFW

$\mathrm{t}=1400 \mu \mathrm{s}$

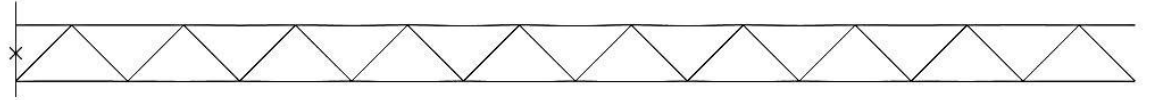

$\mathrm{t}=1700 \mu \mathrm{s}$

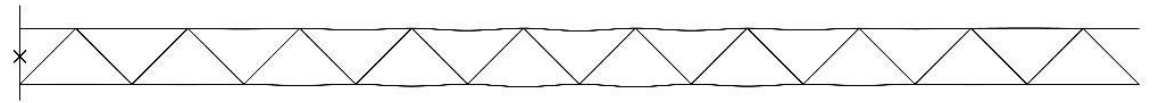

(c) EFW

$\mathrm{t}=2800 \mu \mathrm{s}$

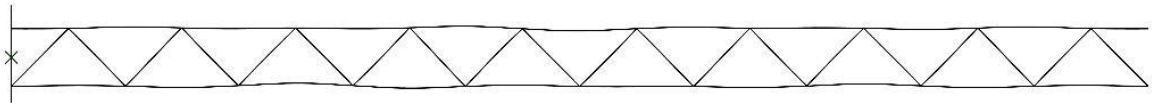

$t=3600 \mu s$

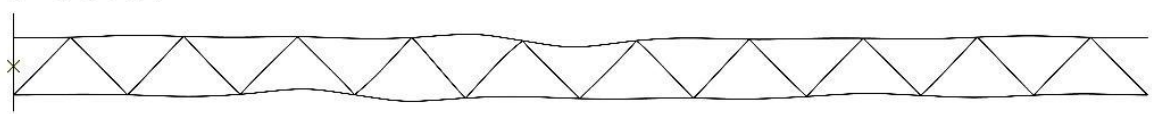

(d) PFW

Figure 3.7: Comparison between the FE and analytical calculations of the reaction force curves of the SS304 representative sandwich columns of (a) the EFW geometric dimensions under V=0.1m/s; (b) the PFW geometric dimensions under V=1.0m/s; Deformation shapes of (c) the EFW obtained from the FE simulation at $\mathrm{t}=1400,1700 \mu \mathrm{s}$, and of (d) the PFW obtained from the FE simulation at $2800,3600 \mu \mathrm{s}$, 
The analytical model is in good agreement with the FE simulations except for the Al6061-T6 sandwich column response of the EFW geometric dimensions. The discrepancy results from local yielding at nodes, which is not accounted for in the analytical model and will be discussed in Section 3.4.3.

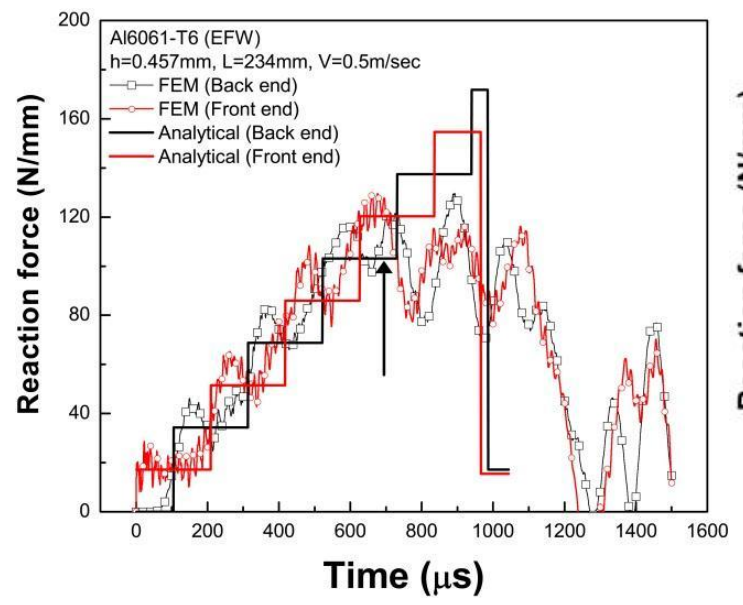

(a) EFW

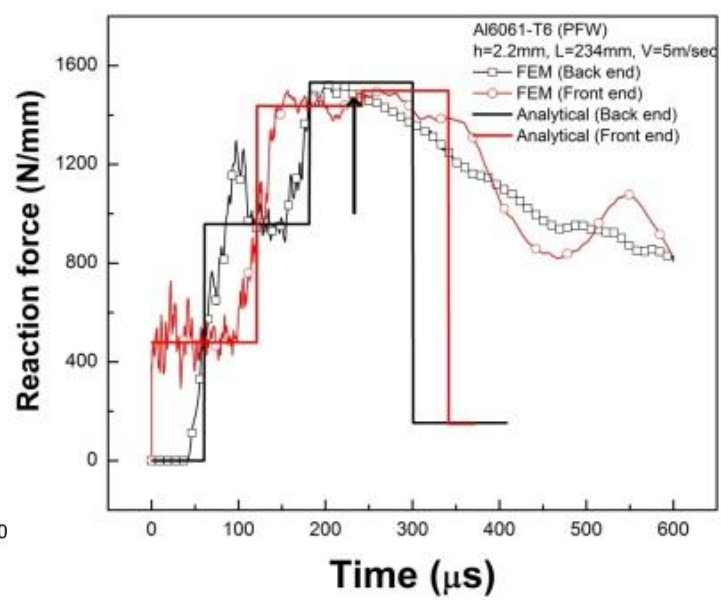

(b) $\mathrm{PFW}$

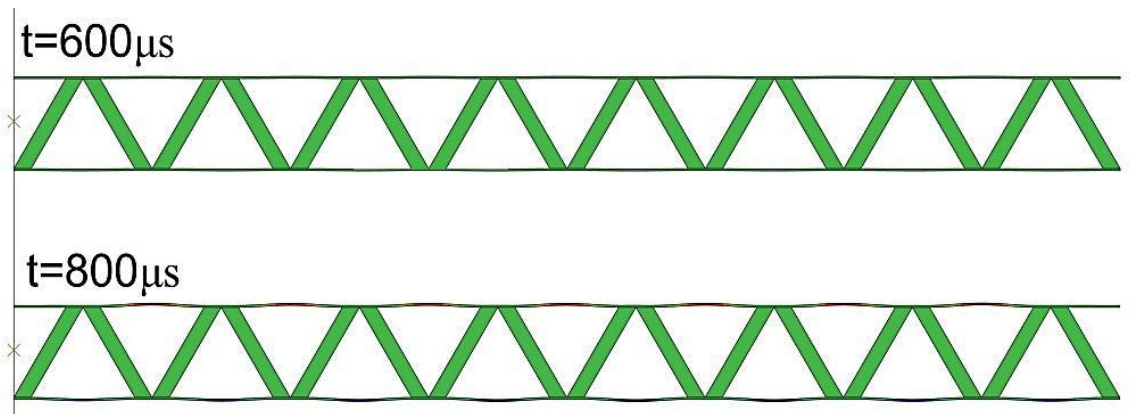

(c) EFW
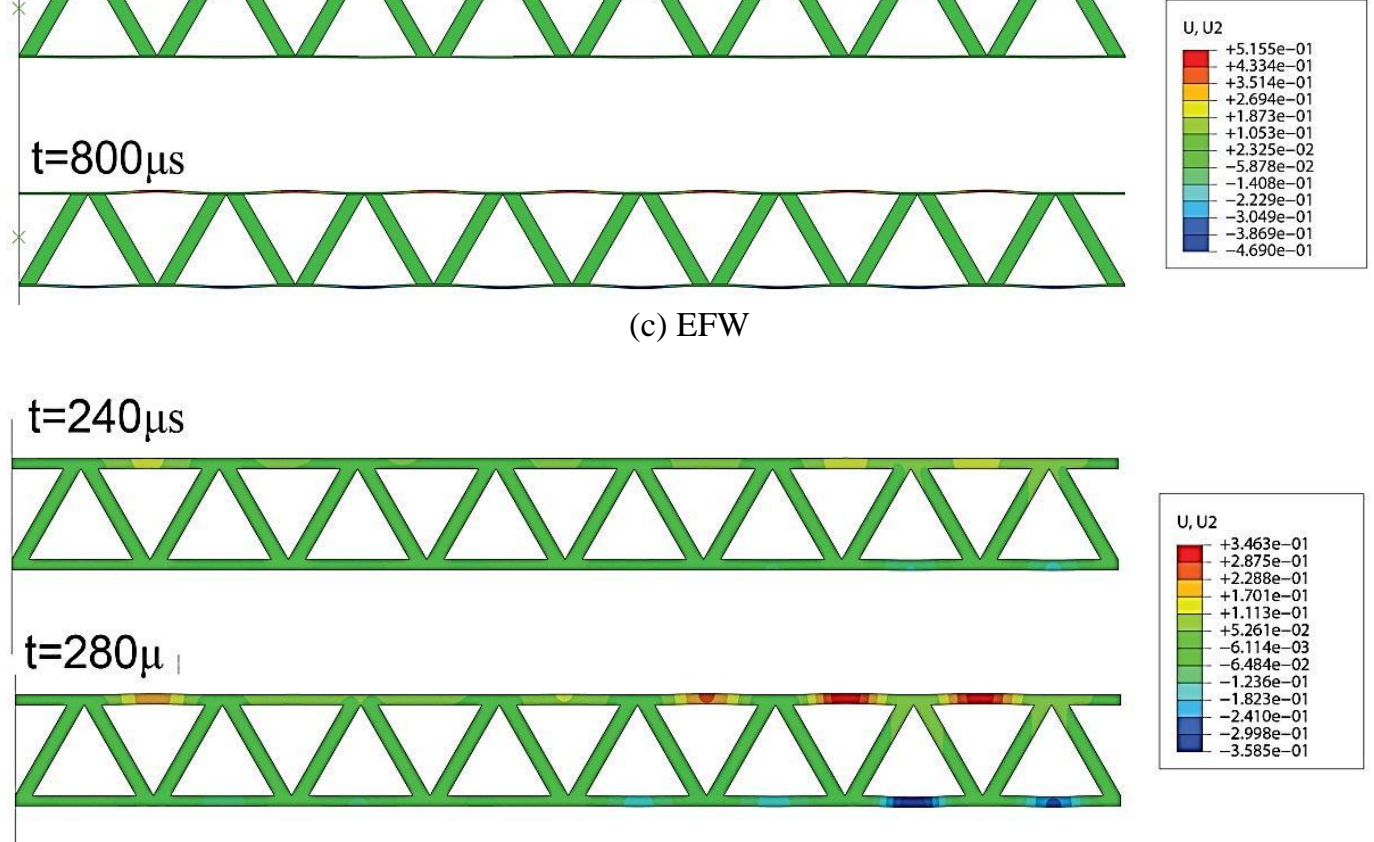

(d) PFW

Figure 3.8: Comparison between FE and analytical calculations for the reaction force curves of the Al6061T6 representative sandwich columns of (a) the EFW geometric dimensions under V=0.5m/s; (b) the PFW geometric dimensions under $\mathrm{V}=5.0 \mathrm{~m} / \mathrm{s}$; Deformation shapes of (c) the EFW obtained from the FE simulation at $\mathrm{t}=600,800 \mu \mathrm{s}$, (d) the PFW obtained from the FE simulation at 240, $280 \mu \mathrm{s}$, 


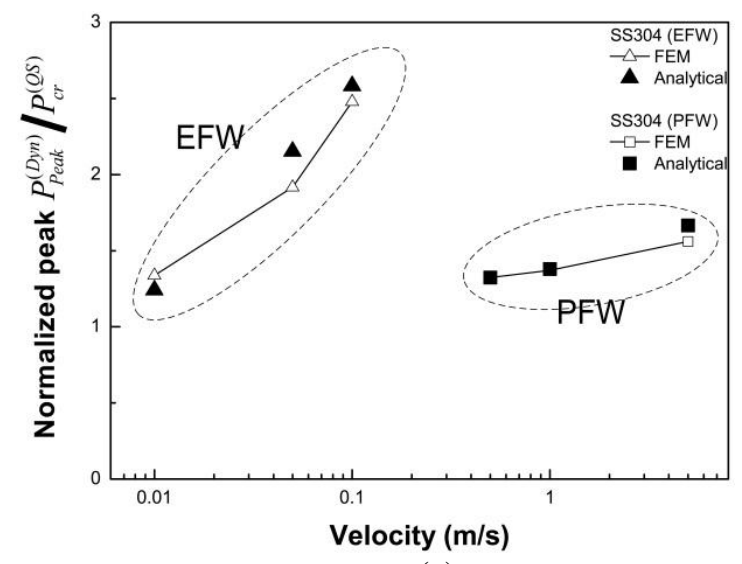

(a)

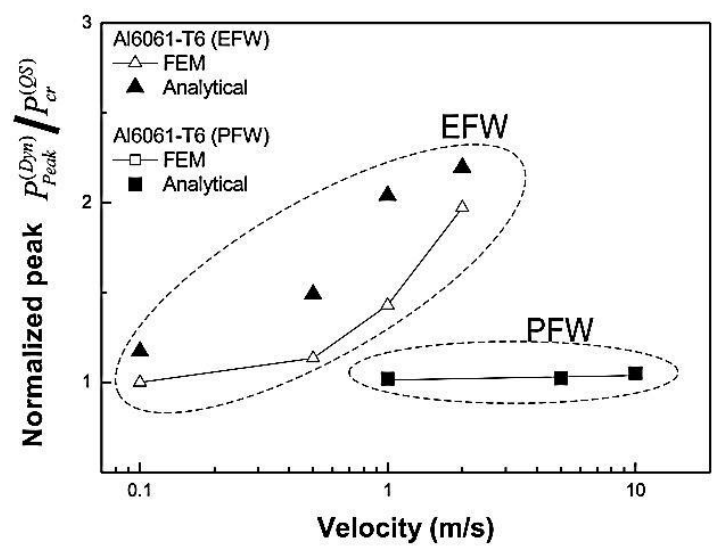

(b)

Figure 3.9: Plots of normalized peak reaction force, $P_{\text {peak }}^{(D y n)} / P_{c r}^{(Q S)}$ vs. V calculated by the FEM and analytical model: (a) SS304 representative sandwich columns; (b) Al6061-T6 representative sandwich columns..

From the four columns shown in Figs. 3.7 and 3.8, it is shown that the maximum peak load in the reaction force curves, $P_{\text {peak }}^{(D y n)}$, is greater than the quasi-static strength, $P_{c r}^{(Q S)}$. It gives clear evidence that dynamic effects enhance column strength. To investigate the effect of rate of loading on dynamic strength, normalized peak load, $P_{\text {peak }}^{(D y n)} / P_{c r}^{(Q S)}$, versus $\mathrm{V}$, which is calculated from both the FEM and analytical model, is plotted in Fig. 3.9. The elevation is more pronounced in the Al6061-T6 and SS304 columns of the EFW geometric dimensions. The elevation in the sandwich columns of the EFW is certainly explained by inertia effects because material strain-rate effects do not influence the response.

On the other hand, the normalized peak load in the representative columns of the PFW geometric dimensions are approximately 1.3 1.5 for the SS304 column under $\mathrm{V}=0.5 \sim 5 \mathrm{~m} / \mathrm{s}$, and 1.0 for the Al6061-T6 one under $\mathrm{V}=1 \sim 10 \mathrm{~m} / \mathrm{s}$, respectively. Note that SS304 is modeled by a strain rate-dependent material while material strain ratedependence in Al6061-T6 is not considered. The range of $P_{p e a k}^{(D y n)} / P_{c r}^{(Q S)}$ of the SS304 
representative column of the PFW geometric dimensions amounts to the dynamic strength enhancement ratio, $\mathrm{R}, 1.29 \sim 1.41$ if approximating equivalent plastic strain rate as $\dot{p} \approx \frac{2}{\sqrt{3}} \frac{V}{L}$. R describes the strain rate-dependence of the parent material, SS304.

$$
R=\frac{\sigma_{y}(\dot{p})}{\sigma_{y}^{0}}=1+\left(\frac{\dot{p}}{D}\right)^{m}
$$

Thus, material strain-rate dependence predominates the dynamic strengthening in the representative SS304 column of the PFW geometric dimensions. Through the investigation in Fig. 3.7 through Fig. 3.9, the analytical model can predict the dynamic strengthening observed in the FE simulations to a good degree of accuracy.

In Fig. 3.10, the transverse deflection of the failed face ligament calculated using the analytical model and FE are compared. Fig. 3.10(a) shows the FE calculation of the transverse deflection at the mid-point of the face ligament of the SS304 representative sandwich column of the EFW geometric dimensions under $\mathrm{V}=0.1 \mathrm{~m} / \mathrm{s}$. The motion oscillates with a small amount of amplitude until the load drop point at $\mathrm{t}=1300 \mu \mathrm{s}$. After that, significant increase in the transverse displacement leading to plastic dissipation is observed. Therefore, the plastic deformation results from the excessive growth of transverse deflection. The initiation time of plastic dissipation $(\mathrm{t}=1470 \mu \mathrm{s})$ is obtained from an energy balance plot not presented here. The FE observation in Fig. 3.10(a) is consistent with Jones' [33] and Hoff's [38] findings on a simple column under dynamic compression in which an oscillating motion is found as long as the axial force is smaller than Euler buckling load, $\mathrm{P}<\mathrm{P}_{\mathrm{E}}$, and the motion is unbounded if $\mathrm{P}>\mathrm{P}_{\mathrm{E}}$. 


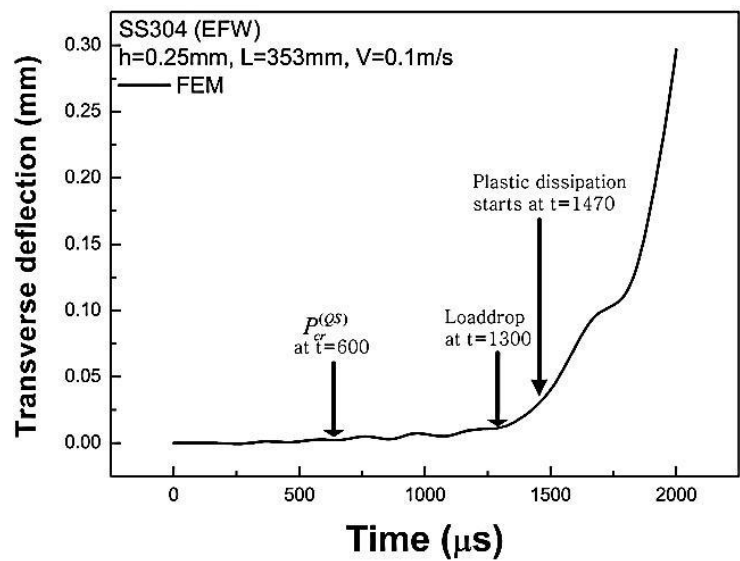

(a)

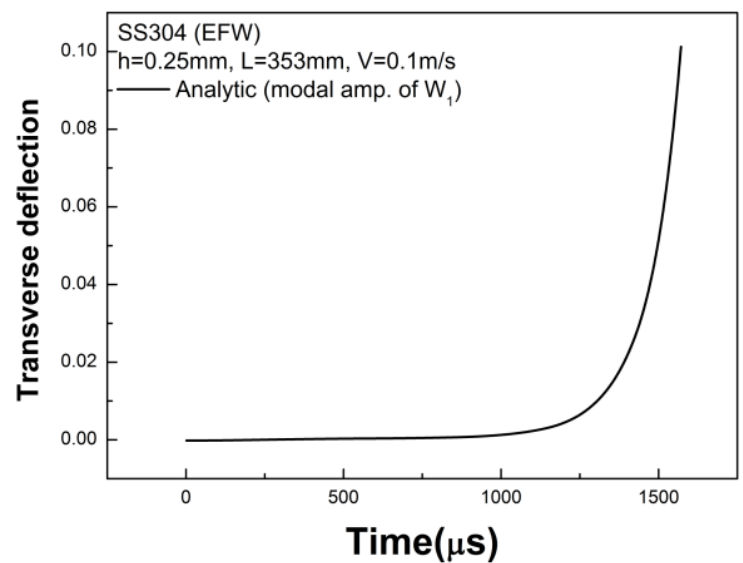

(b)

Figure 3.10: Transverse motion at the midpoint of a failed face ligament of the SS304 representative sandwich column of the EFW geometric dimensions under V=0.1m/s: (a) FEM; (b) analytic model.

In Fig. 3.10(b), the transverse motion of the failed face sheet ligament is obtained from the analytical model. The deflection at a midpoint can be given in terms of the modal amplitude of the first mode, $\mathrm{W}_{1}$. Consistently with the FE result as shown in Fig. 3.10(a), an appreciable increase in the modal amplitude is not found until $t=1000 \mu$ s in Fig. 3.10(b). However, the $\mathrm{W}_{1}$ mode grows significantly after this point.

In Fig. 3.11, the effect of column length, L, on the dynamic face wrinkling failure is investigated by comparing the responses of two sandwich columns having different column length L: One column is the SS304 sandwich column of the EFW geometric dimensions, whereas the counterpart is one having the same geometric dimensions except for column length which is twice as long $(\mathrm{L}=707 \mathrm{~mm})$. The two columns have the same quasi-static strength. Fig. 3.11(a) and (b) show the FE results of reaction force-time history curves of the two columns, which are plotted in the same range of the abscissa and ordinate. The shorter column fails at around $t=1400 \mu$ s with normalized peak reaction forces, $P_{\text {peak }}^{(D y n)} / P_{c r}^{(Q S)}=2.5$ while the longer one at around $\mathrm{t}=2300 \mu \mathrm{s}$ with $P_{\text {peak }}^{(D y n)} / P_{c r}^{(Q S)}=2.0$. Likewise, the responses of the two columns obtained from the 


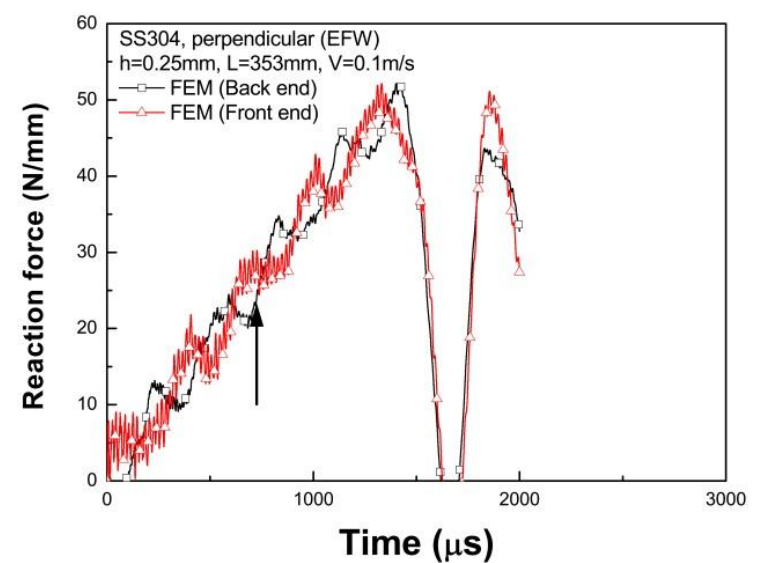

(a) $\operatorname{FEM}(\mathrm{L}=353)$

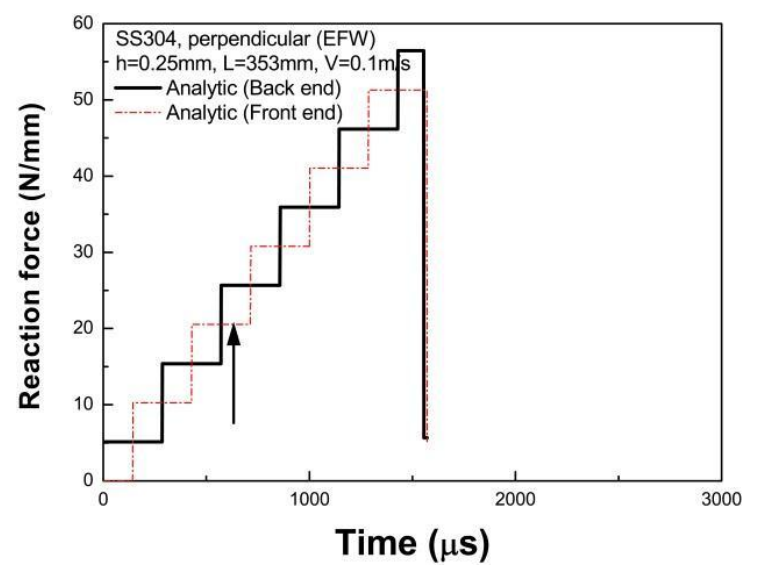

(c) Analytical $(\mathrm{L}=353)$

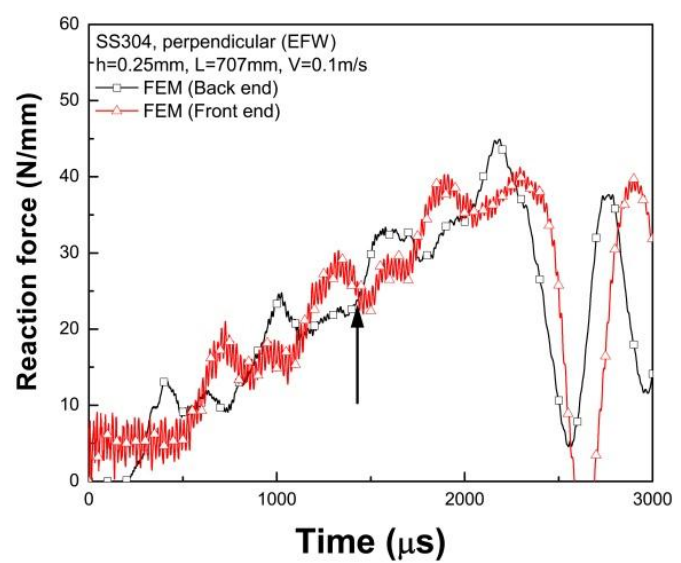

(b) FEM ( $\mathrm{L}=707)$

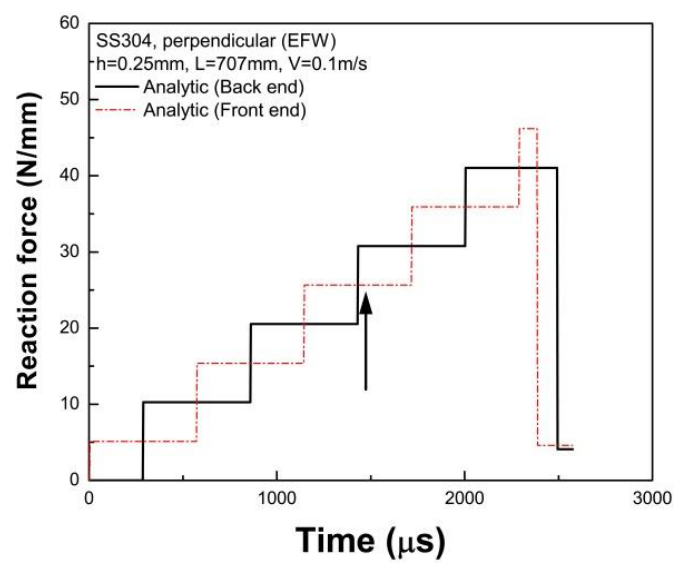

(d) Analytical (L=707)

Figure 3.11: The effect of column length L on the dynamic response of SS304 corrugated core sandwich columns of the EFW geometric parameters having the same static strength, $P_{c r}^{(Q S)}$ : (a) FE calculation for the short column of $\mathrm{L}=353 \mathrm{~mm}\left(P_{\text {peak }}^{(D y n)} / P_{c r}^{(Q S)}=2.5\right.$ ); (b) FE calculation for the longer column of $\mathrm{L}=707 \mathrm{~mm}$ $\left(P_{\text {peak }}^{(D y n)} / P_{c r}^{(Q S)}=2.0\right)$; (c) Analytical prediction for the short column of $\mathrm{L}=353 \mathrm{~mm}$; (d) Analytical prediction for the longer column of $\mathrm{L}=707 \mathrm{~mm}$..

analytical model are shown in Figs. 3.11(c) and (d), giving a good agreement with the FE results in Figs. 3.11(a) and (b). Both the analytical and FE calculations demonstrate that the two elastic columns in dynamic compression reveal neither linearly-scaled responses nor the same dynamic strength. In general, the dynamic strength of a compressive member is dependent on the shape of pulses as well as loading duration. That is because the dynamic strength of compressive members pertains to not only the intensity of loading but also the duration for which they are exposed to compression. In this regard, 
face wrinkling response under dynamic compression was influenced by overall sandwich column geometries such as $\mathrm{L}$ and $\bar{\rho}$ even though they have the same face sheet thickness, h, and face ligament span length, $2 \ell \cos \omega$, as shown in Fig. 3.11.

In Fig. 3.12, the effects of geometric imperfections in the Al6061-T6 and SS304 columns designed with the EFW geometric dimensions are investigated by the FEM and analytical model. For the FE sandwich column models having geometrically imperfect face sheets, one eigenmode from additional static buckling analysis is superimposed on the perfect mesh with magnitudes of $\xi_{i}^{(f)}=0,0.01 \mathrm{~h}, 0.05 \mathrm{~h}$. For example, the first few modes generated from an eigen-buckling analysis for the Al6061-T6 columns of the EFW geometric dimensions are face wrinkling modes as shown in Fig. 3.13(b) and (c) (In general, the number of the face wrinkling modes to be seen from the lowest mode is dependent on the number of unit-cells.). The mode to be inserted into dynamic FEA for imperfect columns is selected such that maximum displacement in the eigenmode occurs at the failed face ligament in the dynamic FEA of sandwich columns of perfect geometry (Fig. 3.13(a)). In the Al6061-T6 case of the EFW, Mode \#7 in Fig. 3.13(c) rather than Mode \#1 is selected to be inserted into the dynamic FE simulation for the imperfect columns. Eventually, dynamic FE simulations for imperfect sandwich columns are performed on the FE model in which nodal positions are relocated with the amount of $\xi$ from the perfect sandwich column mesh.

On the analytical side, the analytical model for the geometrically imperfect face sheet ligaments $\left(\xi_{i}^{(f)}=0.01 \mathrm{~h}, 0.05 \mathrm{~h}\right)$ defines the imperfection magnitude $\xi_{1}$ in Eqn. (3.12) whereas the analytical model for the perfect geometry $\left(\xi_{i}^{(f)}=0.0\right)$ employs the procedure for the asymptotic analysis in Section 3.3.5. 


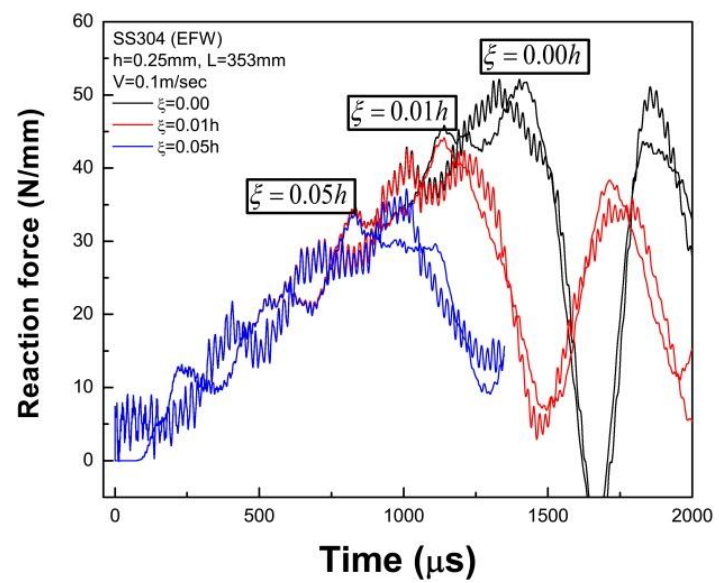

(a)

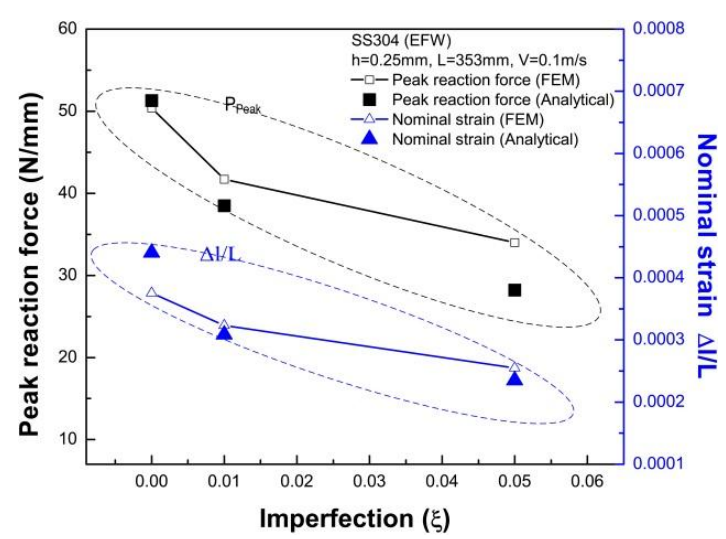

(c)

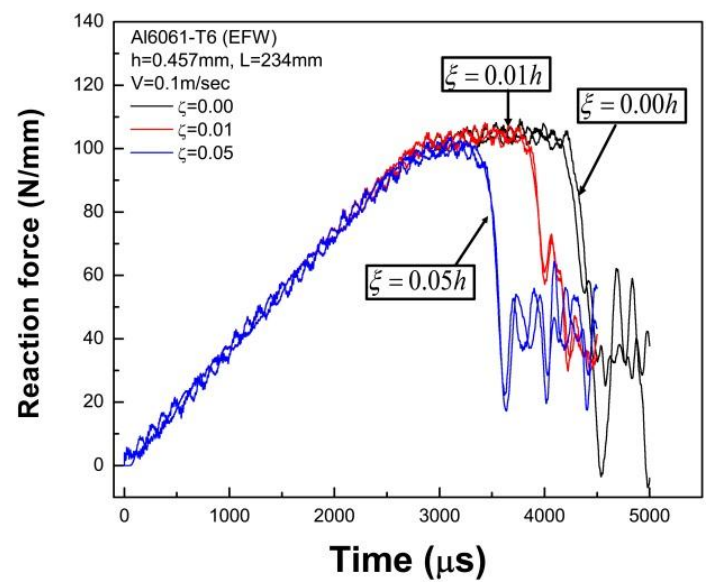

(b)

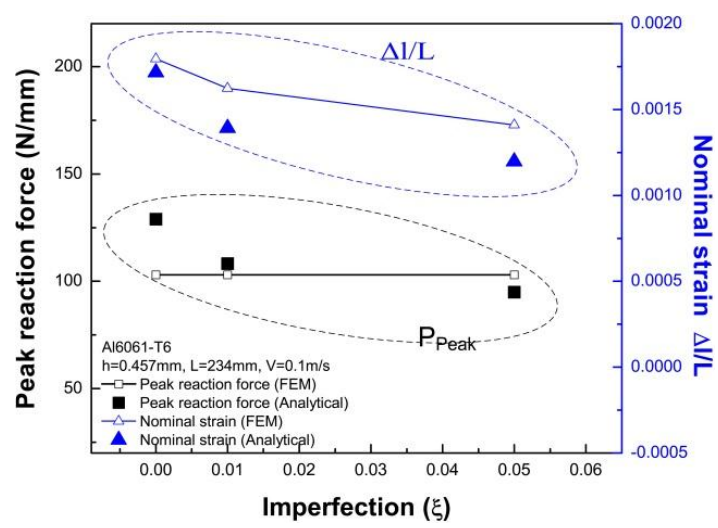

(d)

Figure 3.12: Influence of geometric imperfections. Reaction force versus time history curves calculated from FEM of (a) SS304 representative sandwich column of the EFW geometric dimensions under V=0.1 $\mathrm{m} / \mathrm{s}$, (b) Al6061-T6 representative sandwich column of the EFW geometric dimension under $V=0.1 \mathrm{~m} / \mathrm{s}$; And the analytical model are compared against the FEA on the imperfection sensitive dynamic peak load and nominal strain at load drop of (c) the SS304 column and (d) the Al6061-T6 column.

In Figs. 3.12(a) and (b), the reaction force-time history curves obtained from the FE simulations are plotted for the SS304 and Al6061-T6 sandwich columns of the EFW geometric dimensions having different amount of imperfections subject to $V=0.1 \mathrm{~m} / \mathrm{s}$, respectively. With increasing $\xi_{i}^{(f)}$, the SS304 sandwich columns show the reduction in peak reaction force and nominal strain. In contrast, the imperfection affects the duration of plateau in the Al6061-T6 sandwich column of the EFW geometric dimension. The 


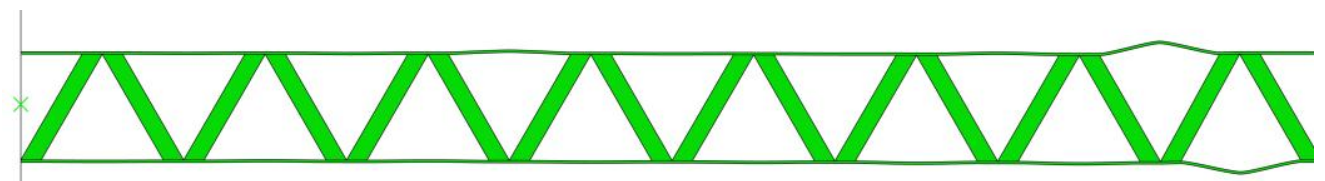

(a)

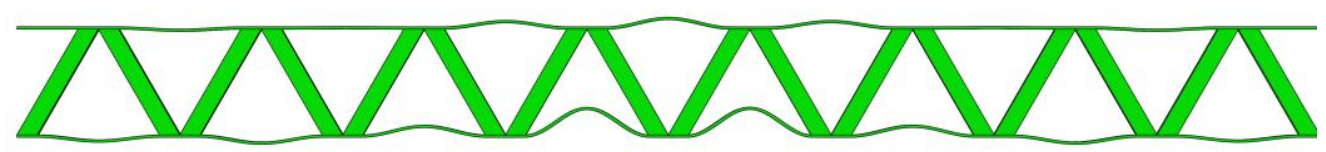

(b)

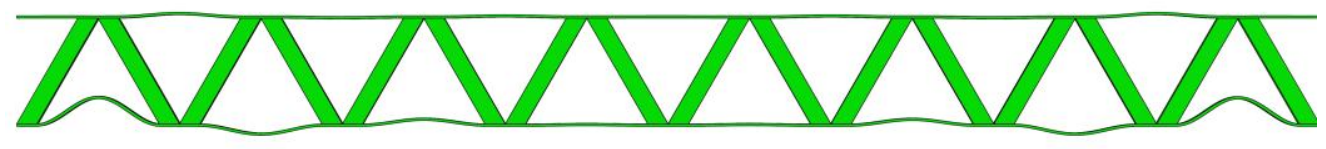

(c)

Figure 3.13: (a) Dynamic simulation of the representative Al6061-T6 sandwich column of the EFW geometric dimensions (perfect geometry); (b) The first eigenmode; (c) The $7^{\text {th }}$ eigenmode.

plateau found in the reaction curve of the Al6061-T6 sandwich column will be discussed in the next section.

In Figs. 3.12(c) and (d), the analytical predictions for the imperfection-dependent peak reaction forces and nominal strains are compared against the FE simulations. Nominal strain, $\Delta L / L$, is defined as the ratio of compressed distance $\Delta \mathrm{l}$ at the time of load drop to column length $\mathrm{L}$. The analytical model agrees well with the FEM for the imperfection-dependent response of the SS304 representative column as shown in Fig. 3.12(c). However, the analytical model does not capture a tendency observed from the FE simulations for the Al6061-T6 column of the EFW geometry, in which the peak reaction force is invariant to imperfection magnitude, $\xi_{i}^{(f)}$. Instead, a decreasing tendency of nominal strain with increasing magnitude of imperfections is found from both the FEM and analytical model. 


\subsubsection{Limitation and Efficiency of the Analytical Model}

One limitation of the analytical model is found in the plateau response of the Al6061-T6 representative sandwich columns of the EFW geometric dimensions. In this section, a source causing such phenomenon is addressed.

In Fig. 3.14(a)(b)(c), the reaction force curves of the Al6061-T6 column under $\mathrm{V}=0.1,0.5,1.0 \mathrm{~m} / \mathrm{s}$ are shown, respectively. The curves are replotted on a normalized time scale, Vt/L, in Fig. 3.14(d). As shown in the figures, the plateau response is more pronounced at lower velocities. To examine energy conversion during the plateau response, an energy balance plot for $\mathrm{V}=0.1 \mathrm{~m} / \mathrm{s}$ is shown in Fig. 3.15. External work $\left(\mathrm{W}_{\text {ext }}\right)$ generated by axial compression of the Al6061-T6 sandwich column is stored in the column as a form of elastic strain energy (EE) until plastic dissipation (PD) starts to appear at $\mathrm{t}=3400 \mu \mathrm{s}$. Then it is followed by a significant increase of kinetic energy (KE) and plastic dissipation (PD), which is coincident with the load drop. The sequence of PD, KE, load drop for the candidate SS304 sandwich column with EFW geometric dimensions is highlighted in Fig. 3.10. In the SS304 column, excessive deformation of an elastic face sheet ligament leads to the unstable load drop followed by plastic dissipation whereas local plastic deformation at nodes makes the plateau response of the Al6061-T6 column followed by load drop.

The local plastic deformation at nodes in the plateau region for the Al6061-T6 geometries can be confirmed by a contour plot of equivalent plastic strain at $t=3883 \mu$ s in Fig. 3.16. The lower part of the figure explains local plastic deformation on the outer fiber of the face sheet near the nodes between a core and face sheets. Therefore, local plastic deformation at nodes causes a plateau response, which cannot be accounted for by 
the analytical model because local plastic deformation violates the kinematic assumption of the analytical model.

Through about forty FE simulations regarding Al6061-T6 EFW sandwich columns under relatively low velocities $(\mathrm{V}: 0.1 \sim 1 \mathrm{~m} / \mathrm{s})$ in addition to the results presented here, the plateau response is usually observed in elastically face wrinkled sandwich columns compressed at a comparatively low velocity $(\mathrm{V} \sim 0.1 \mathrm{~m} / \mathrm{s})$. Even though the valid range of the proposed analytic model is not quantified here, it can be postulated that local plastic deformation at nodes associated with the plateau response is susceptible to sandwich columns having stubby elastic faces with large oscillating amplitude, which might be related to the slenderness ratio of face ligaments, corrugation angles, imperfections, and core inertia etc. Comparing the slenderness ratios of the face ligaments of the Al6061-T6 and SS304 sandwich columns of the EFW geometric dimensions, the slenderness ratio of a face ligament of the SS304 column is much greater than that of the Al6061-T6 columns. The elastic face ligaments with high slenderness ratio allow large elastic deflection before local plastic deformation. As an extreme case, an Al6061-T6 monolithic column in Fig. 2.13 in Chapter 2, which has a high slenderness ratio, does not reveal such the response.

This can be proved simply by the strength-of-materials approach. The axial stress at the outer fiber of a face sheet ligament subject to an axial load, $\mathrm{P} / 2$, is given by

$$
\sigma_{\text {outer }}(x)=\frac{P}{2 h}+\frac{E}{\left(1-v^{2}\right)}\left(\left|w^{\prime \prime}(x)\right|\right)(h / 2)
$$

Therefore, the axial stress at the position corresponding to a node is given as

$$
\sigma_{\text {outer }}(0)=\frac{P}{2 h}+\frac{\left(E\left|w^{\prime \prime}(0)\right|\right)(h / 2)}{\left(1-v^{2}\right)}
$$

Assuming one mode approximation for the transverse deflection of a face ligament 
with a shape function of $W_{1}^{(f)}(x)=\frac{1}{2}\left(1-\cos \frac{2 \pi x}{(2 l \cos \omega)}\right)$,

$$
\begin{gathered}
w(x, t)=c_{1}(t) \cdot W_{1}^{(f)}(x) \\
\left.\frac{d^{2} w}{d x^{2}}\right|_{x=0}=\left.c_{1} \frac{d^{2} W_{1}^{(f)}}{d x^{2}}\right|_{x=0}=\frac{c_{1}}{2}\left(\frac{2 \pi}{(2 \ell \cos \omega)}\right)^{2}
\end{gathered}
$$

After substituting Eqn. (3.20b) into Eqn. (3.19), a condition of local plastic deformation for a given load, $\mathrm{P} / 2$, can be determined by

$$
\frac{P}{2}+\frac{\pi^{2} E}{\left(1-v^{2}\right)} \frac{c_{1} h^{2}}{(2 \ell \cos \omega)^{2}}<\frac{h \cdot \sigma_{Y}}{\sqrt{1-v_{P}+v_{P}^{2}}}
$$

In Eqn. (3.21), the local deformation at nodes is dependent on the second term of the left hand side. That is, for a given load $\mathrm{P} / 2$, a large amplitude in the transverse deflection, $\mathrm{c}_{1}$, coupled with a small slenderness ratio $(2 l \cos \omega) /(h)$, results in the local plastic deformation.

Nevertheless, the proposed model is quite attractive from an efficiency point of view. For example, it took approximately one hundred hours for a FE simulation of dynamic compression of an Al6061-T6 corrugated core sandwich columns consisting of 64 unit-cells $(\mathrm{L}=1877 \mathrm{~mm})$ at a compression rate of $\mathrm{V}=0.1 \mathrm{~m} / \mathrm{s}$. Accordingly, it is unreasonable to employ FEM for a preliminary periodic core sandwich design tool. However, the analytical model approximates the response within a few seconds. At an initial design stage, there is a necessity to probe the dynamic response for sandwich columns with various geometric dimensions. Under the circumstances where there is no simple prediction method considering dynamic effects, the analytical models presented in Chapters 2 and 3 can produce an approximate response quickly in a design space, which could be complementary to the FEM. 


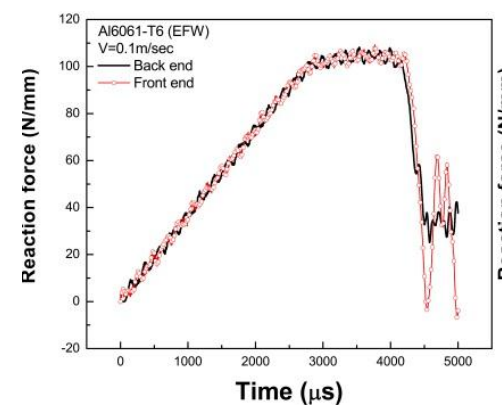

(a) $\mathrm{V}=0.1 \mathrm{~m} / \mathrm{s}$

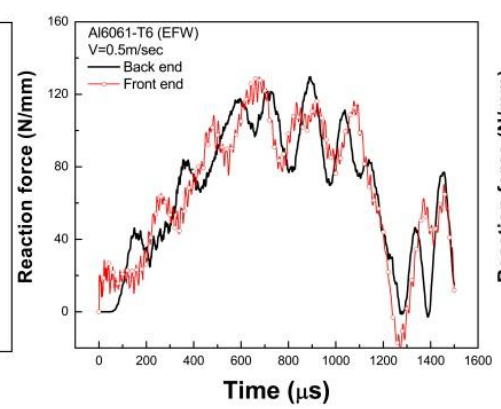

(b) $\mathrm{V}=0.5 \mathrm{~m} / \mathrm{s}$

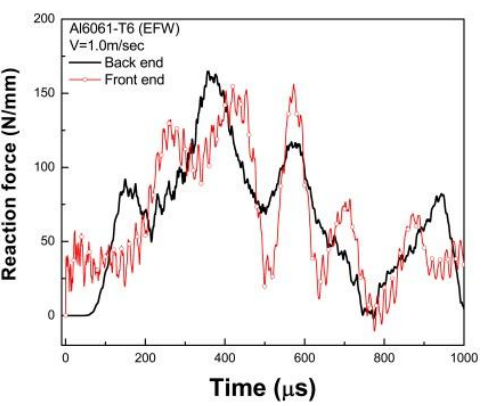

(c) $\mathrm{V}=1.0 \mathrm{~m} / \mathrm{s}$

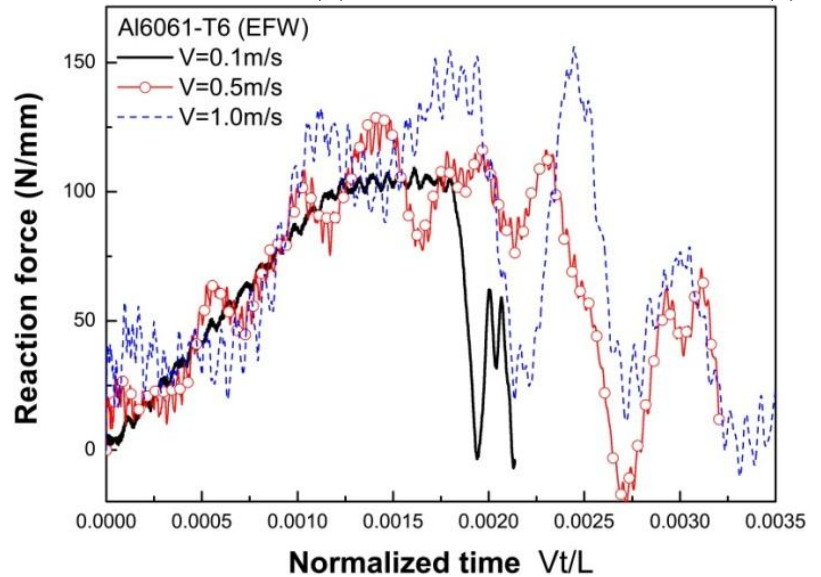

(d)

Figure 3.14: Reaction force-time history curves at both front and back of an Al6061-T6 representative corrugated core sandwich column of the EFW geometric dimensions under (a) V=0.1m/s; (b) V=0.5m/s; (c) $\mathrm{V}=1.0 \mathrm{~m} / \mathrm{s}$; (d) The reaction force versus normalized time histories for the three compression rates.

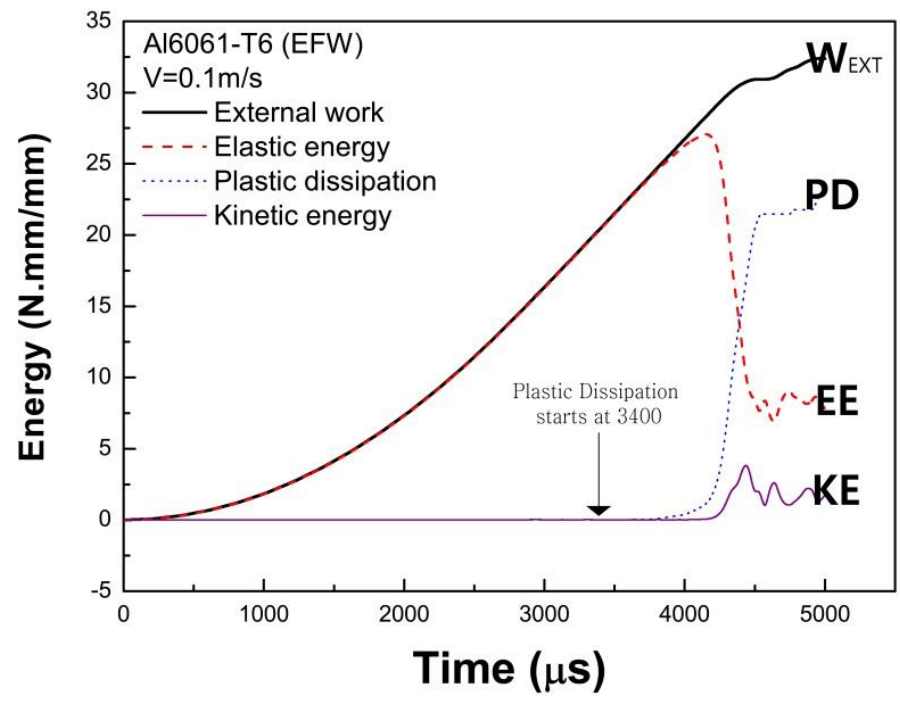

Figure 3.15: Energy balance plot of the Al6061-T6 representative sandwich column of the EFW geometric dimensions under $V=0.1 \mathrm{~m} / \mathrm{s}$. External work (Wext) is partitioned into 1) Elastic strain energy (EE), 2) Plastic dissipation (PD), and 3) Kinetic energy (KE). 

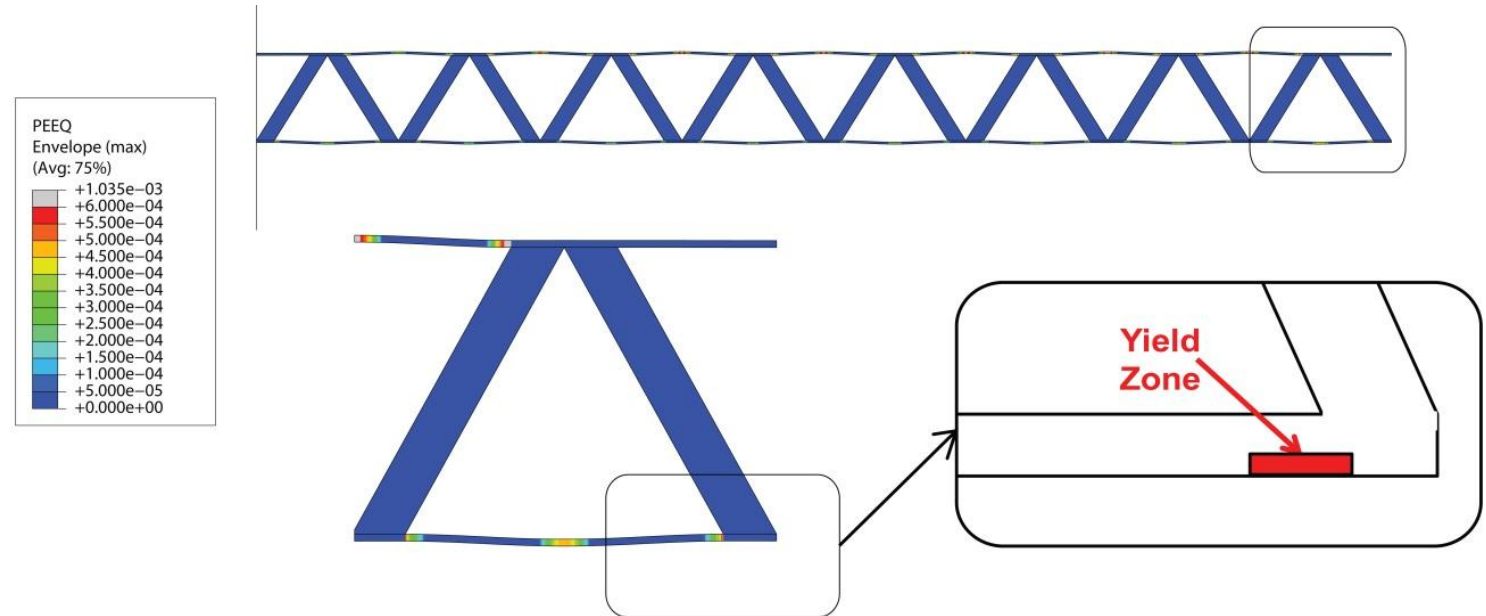

Figure 3.16: Contour of equivalent plastic strain, p, of the Al6061-T6 representative sandwich column of the EFW geometric dimensions under $\mathrm{V}=0.1 \mathrm{~m} / \mathrm{s}$ at $\mathrm{t}=3883 \mu \mathrm{s}$. The lower explains local plastic deformation on the outer fiber of the face sheet near a node between face sheets and a core.

\subsection{CHAPTER SUMMARY}

An analytical model for face wrinkling failure under dynamic compression of corrugated core sandwich columns is proposed. The model calculates a face ligament transverse deflection based on the consideration of a face ligament as an Euler-Bernoulli beam-column, continuum approximation of the discrete cores with wave propagation theory.

The fundamental assumption for axial force history in a face ligament was verified by comparing the apparent elastic stress wave speeds with the FE measurements and analytic expression. It reveals that the wave speed in corrugated core sandwich columns is a function of not only material properties but also sandwich geometric parameters.

As demonstrated in Section 3.4.2, the proposed analytical model for face wrinkling failure under dynamic compression of corrugated core sandwich columns successfully reproduced most of the observations in the FE simulations. However, there is a limitation 
that the model is not capable of predicting the plateau response which has been seen in Al6061-T6 sandwich columns of the EFW geometric dimensions, especially compressed at a low velocity. It is demonstrated that the plateau response results from local plastic deformation at nodes.

The efficiency and reasonable accuracy of the proposed model is noteworthy. When combined with the global buckling analytical model presented in Chapter 2, the face-wrinkling analytical model proposed in this chapter can be utilized for understanding competition of failure modes and optimal designs of corrugated core sandwich columns under dynamic compressive loading. 


\section{CHAPTER 4}

\section{AN ANALYTICAL APPROACH \\ FOR THE PREDICTION OF LOCAL PLATE BUCKLING}

\subsection{INTRODUCTION}

An analytical model predicting the dynamic failure of plates with a large dimension in the longitudinal direction compressed at a constant rate of $\mathrm{V}$ is proposed. The

compression rate is in the low velocity region such that $V /\left(c_{e l}^{(\text {para })} \varepsilon_{Y}\right)<1$ as in Chapters 2 and 3. The work in this chapter begins with the hypothesis that an analytical approach can be an alternative methodology to approximate dynamic local plate buckling of either core plates or face plates of corrugated core sandwich columns compressed parallel-to-corrugations at a constant velocity.

Plates compressed at a constant velocity cause several wrinkles with a regular pattern to form, and one of the wrinkles grows excessively to a failure from the FE observations presented subsequently. Accordingly, the proposed model considers an imaginary patch plate (predefined kinematic displacement field) on the long plate so as to encompass the wrinkle, and calculates the out-of-plane displacement in the patch plate till load-drop. Similar to the previous two chapters, the approach is based on the theory of stress wave propagation for estimating in-plane force components in the patch plate. Lastly, the approach will be verified by Finite Element calculations through some examples. 
This chapter is organized as follows: First, dynamic response of several corrugated core sandwich columns compressed parallel-to-corrugations is observed via FEM in Section 4.2. Second, an analytical approach for local plate buckling is proposed based on the observed dynamic phenomena in Section 4.3. Last, the proposed approach is verified with FE simulations, and limitations are discussed in Section 4.4.

\subsection{FE OBSERVATIONS OF LOCAL PLATE BUCKLING OF SANDWICH COLUMNS}

\subsubsection{FE Model}

Finite Element Analysis (FEA) for SS304 corrugated core sandwich columns in compression at constant velocities is performed to investigate the dynamic local plate buckling response. A commercial FE package, ABAQUS/Explicit, is employed for the numerical investigation of the four cases listed in Table 4.1. The FE SS304 sandwich columns are constructed as described in Chapter 2. One unit cell with symmetric boundary conditions imposed on their lateral sides, which is a unit cell in the perpendicular-to-corrugation direction, is extruded to the desired length, L (Fig. 2.10(b)).

Conventional shell elements with reduced integration (S4R) are meshed on the face sheets and cores. The parent material SS304 is considered as an elastic-plastic material with bilinear strain hardening and strain-rate hardening as mentioned in the previous

Table 4.1. Geometric dimensions and velocity of FE sandwich column models for the investigation.

\begin{tabular}{cccccccc}
\hline $\begin{array}{c}\text { FE Column } \\
\text { No. }\end{array}$ & $\begin{array}{c}\mathrm{h} \\
(\mathrm{mm})\end{array}$ & $\begin{array}{c}\mathrm{L} \\
(\mathrm{mm})\end{array}$ & $\begin{array}{c}\mathrm{t} \\
(\mathrm{mm})\end{array}$ & $\begin{array}{c}\mathrm{l} \\
(\mathrm{mm})\end{array}$ & $\begin{array}{c}\mathrm{w} \\
(\mathrm{deg})\end{array}$ & $\begin{array}{c}\bar{\rho} \\
(\%)\end{array}$ & $\begin{array}{c}\mathrm{V} \\
(\mathrm{m} / \mathrm{s})\end{array}$ \\
\hline \hline Column I & 0.5 & 353 & 0.675 & 25.0 & 45 & 5.3 & 1.0 \\
Column II & 0.25 & 353 & 0.675 & 25.0 & 45 & 5.3 & 0.5 \\
Column III & 0.25 & 353 & 0.675 & 25.0 & 45 & 5.3 & 5.0 \\
Column IV & 3.75 & 353 & 0.675 & 25.0 & 45 & 5.3 & 5.0 \\
\hline
\end{tabular}


chapters (Fig. 2.11).

\subsubsection{FE Results}

Fig. 4.1 shows the reaction forces vs. time response measured at both ends of the column, and the deformed shapes predicted by FE for column I at three points in time, $\mathrm{t}_{1}=533 \mu \mathrm{s}, \mathrm{t}_{2}=1067 \mu \mathrm{s}, \mathrm{t}_{3}=4000 \mu \mathrm{s}$. The core and lower face are also displayed at those times. Contour plots indicate the displacement component in the y direction, which is normal to the plane of the face sheets. For the time period of $0<\mathrm{t}<600 \mu \mathrm{s}$, the reaction force increases at a constant rate as shown in Fig. 4.1(a), and wrinkles begin to appear in the face sheet as shown in Fig. 4.1(b). At around $t=700 \mu$ s, reaction forces drop suddenly which coincide with the face sheets displaying excessive growth of some wrinkles. Nevertheless, in-plane load of P 600N/mm has been supported for a period until the corrugated core fails at $\mathrm{t}=2200 \mu \mathrm{s}$ globally. Therefore, local plate buckling of the face sheets induces another failure mode resulting in complete loss of structural function.

Figures 4.2 and 4.3 correspond to the analysis of columns II and III, respectively. They have the same geometric dimensions, but are subjected to different compression velocities, $V=0.5 \mathrm{~m} / \mathrm{s}$ and $5 \mathrm{~m} / \mathrm{s}$. Contour plots show the displacement component in the $y$ direction. The FE analysis of column II is for the low loading rate of $\mathrm{V}=0.5 \mathrm{~m} / \mathrm{s}$. Initially, the reaction force of column II increases for the time period of $0<\mathrm{t}<1100 \mu$ s as shown in Fig. 4.2. A slight change of the slope at around the time $t_{1}=1000 \mu \mathrm{s}$ is detected, which might be caused by the appearance of a buckling pattern on the upper and lower face sheets. However, there is no substantial deformation of the core so that the column integrity is maintained without a significant load drop until $t=7000 \mu \mathrm{s}$. After $t=7000 \mu \mathrm{s}$, the 
column fails globally due to the collapse of the core. Conversely, column III in Fig. 4.3, undergoes vertical displacement due to Poisson's effect initially. However, after t=200 $\mu$ s, the reaction forces drop suddenly because of face sheet buckling as shown in the deformed shapes at $\mathrm{t}_{2}=220 \mu \mathrm{s}$. The core has been capable of supporting an in-plane load $\mathrm{P} \sim 500 \mathrm{~N} / \mathrm{mm}$ until it fails in local core plate buckling around $\mathrm{t}=700 \mu$ s. Observe the deformation profiles at $t_{3}=1067 \mu$ s. From Figs. 4.2 and 4.3, local plate buckling induces another failure mode for complete loss of structural function, but the subsequent failure mode leading to the complete loss of structural integrity is different depending on the applied velocities.

Column IV presents the case of initial local plate buckling at core plates as shown in Fig. 4.4. At $0<\mathrm{t}<3000 \mu \mathrm{s}$, a regular buckling pattern starts to appear on the core plate. Significant core plate buckling does not occur until $\mathrm{t}_{2}=3333 \mu$ s as shown in Fig. 4.4. From the deformation shapes at the times, $t_{2}$ and $t_{3}$, one of the buckles grows excessively and then leads to the complete failure due to core shear.

To summarize the FE observations in this section,

(1) Local face plate buckling means a loss of capability to support some of the inplane loads. After that, it weakens the other load-carrying path, which is a core plate, even though the core plate supports in-plane loads for a period of time.

(2) The subsequent failure mode to a local plate buckling can be different depending on compressive velocities. At higher velocities, there is more probability of local plate buckling in the other load-carrying path.

(3) Initially, a regular pattern of buckles is created, and then one of the buckles grows excessively to local plate buckling. 

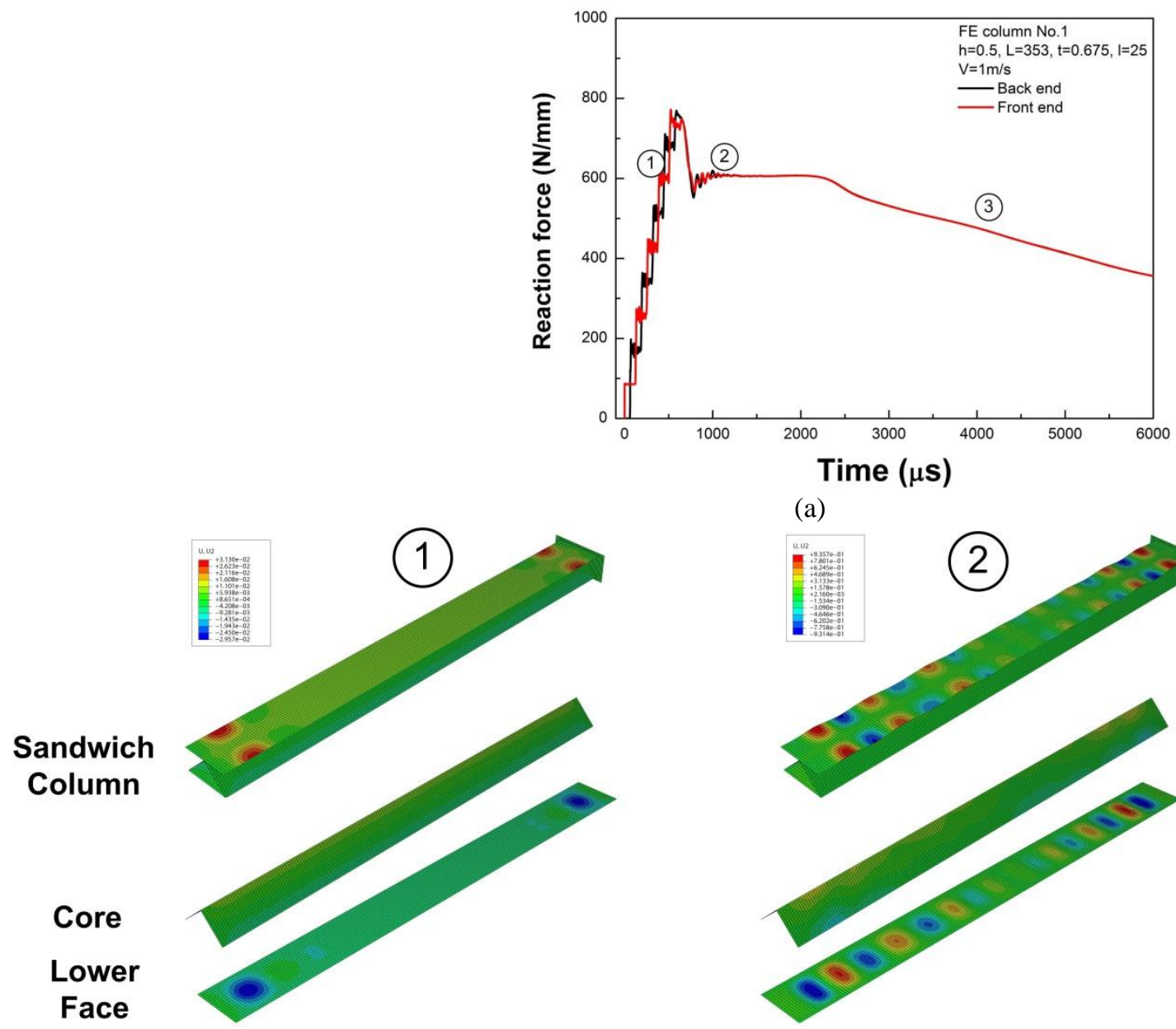

(a)

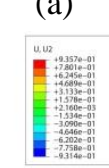

(2)
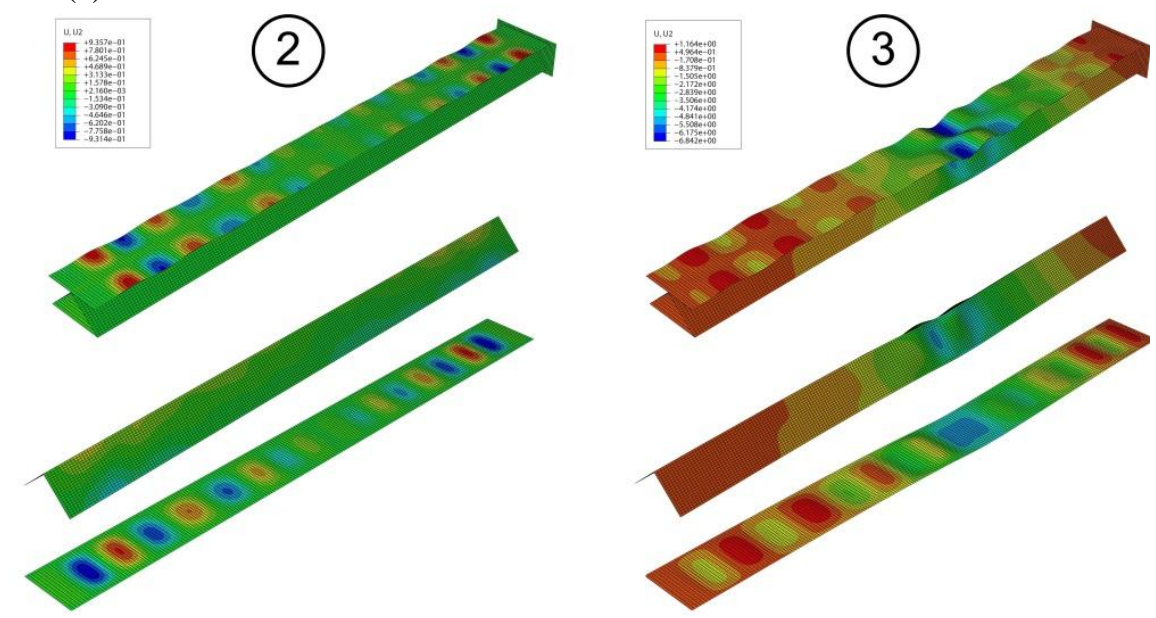

(b)

Figure 4.1: FE column No 1: (a) Reaction forces per unit width at the front and back ends versus time curves; (b) Deformation shapes of sandwich columns at different times on which contours are shown for a vertical displacement component normal to the face sheet planes. Times $t_{1}, t_{2}, t_{3}$ for (1) (2) (3) are 533, 1067, $4000 \mu \mathrm{s}$. 

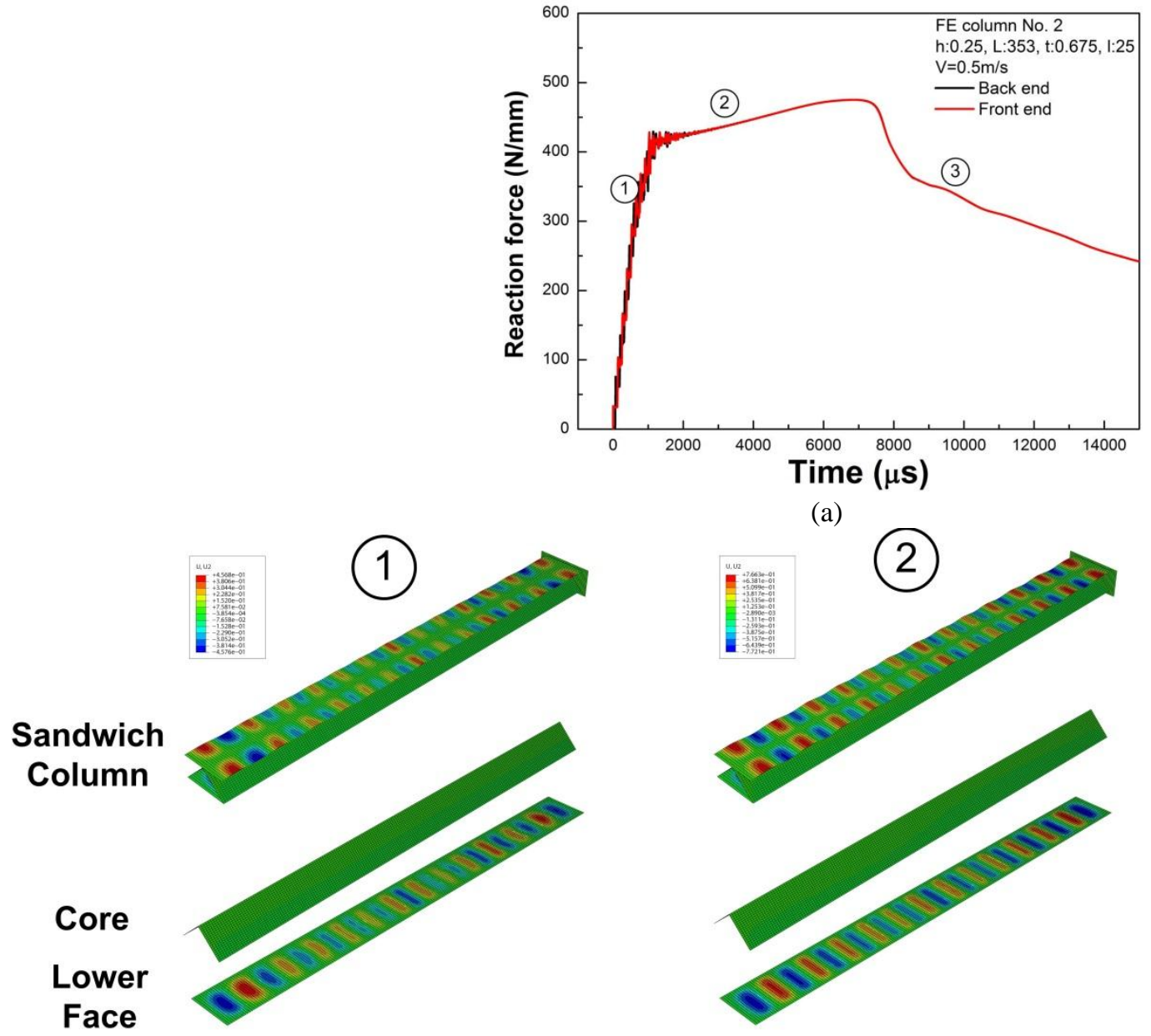

(a)
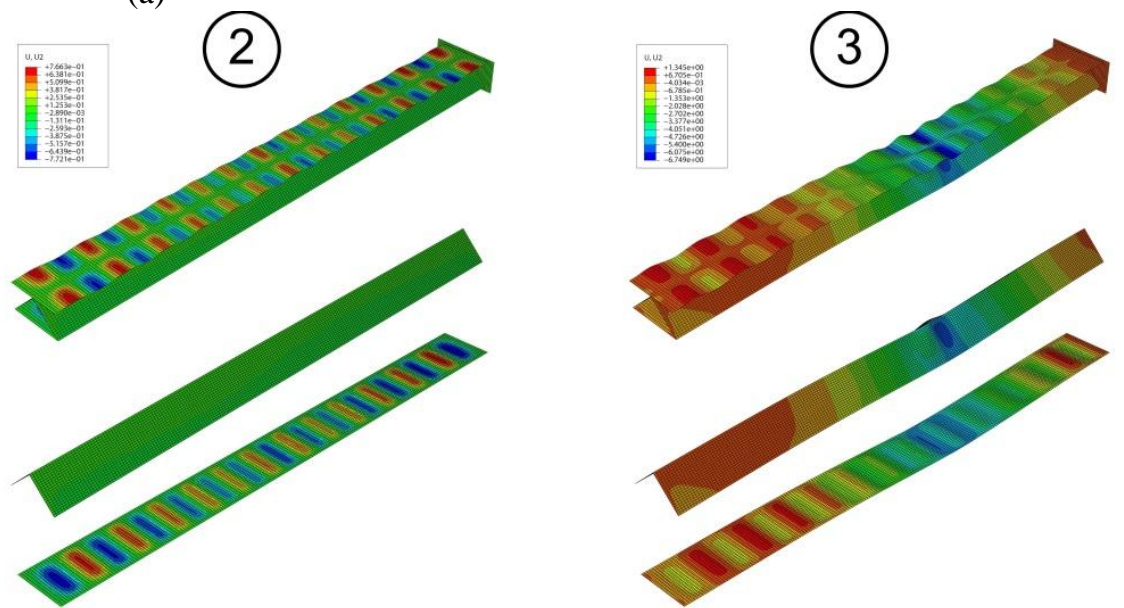

(b)

Figure 4.2: FE column No 2: (a) Reaction forces per unit width at the front and back ends versus time curves; (b) Deformation shapes of sandwich columns at different times on which contours are shown for a vertical displacement component normal to the face sheet planes. Times $t_{1}, t_{2}, t_{3}$ for (1) (2) (3) are 1000, 3000, $9000 \mu \mathrm{s}$. 


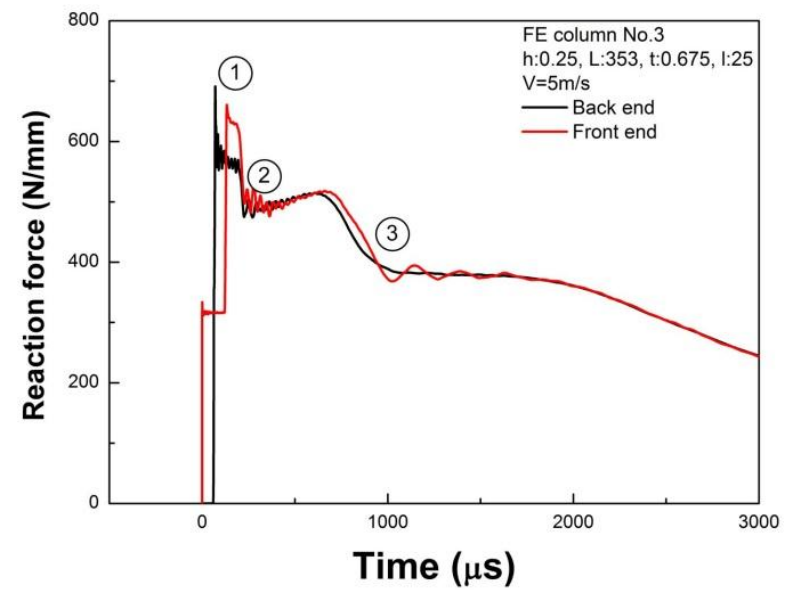

(a)
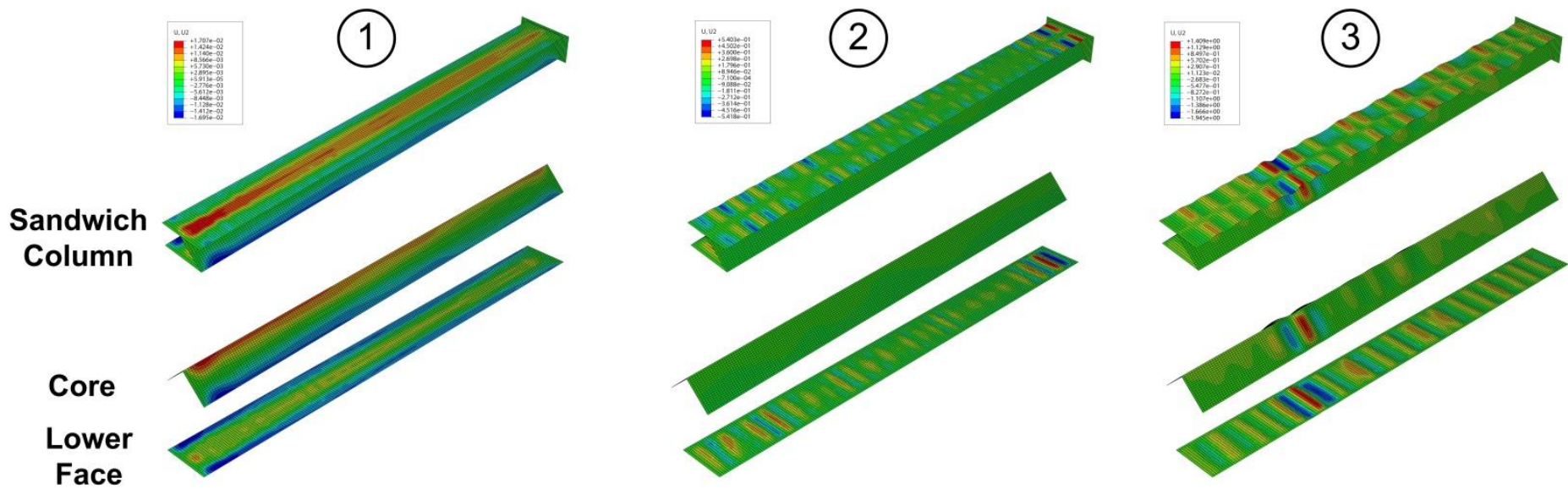

(b)

Figure 4.3: FE column No 3: (a) Reaction forces per unit width at the front and back ends versus time curves; (b) Deformation shapes of sandwich columns at different times on which contours are shown for a vertical displacement component normal to the face sheet planes. Times $t_{1}, t_{2}, t_{3}$ for (1) (2) (3) are 133, 266, $1067 \mu \mathrm{s}$. 


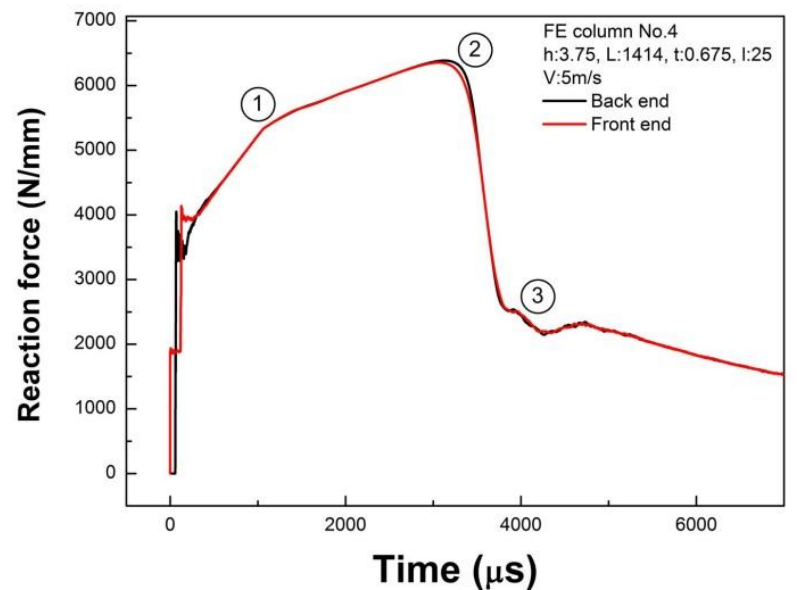

(a)
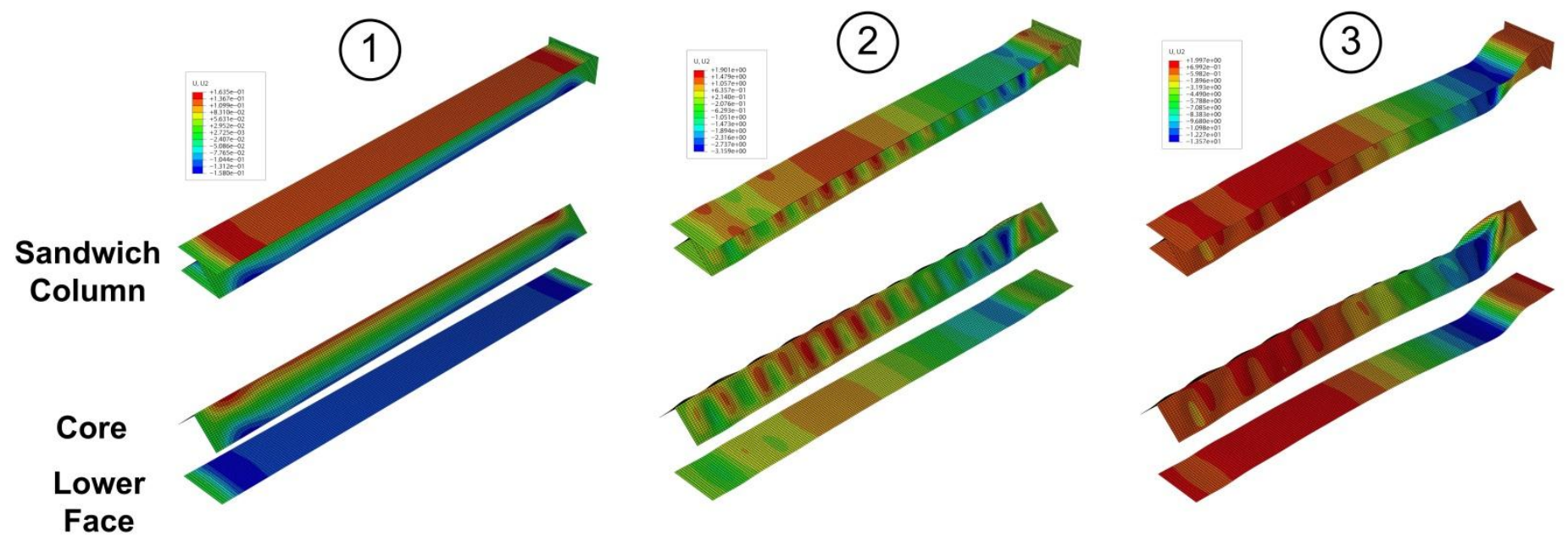

(b)

Figure 4.4: FE column No 4: (a) Reaction forces per unit width at the front and back ends versus time curves; (b) Deformation shapes of sandwich columns at different times on which contours are shown for a vertical displacement component normal to the face sheet planes. Times $t_{1}, t_{2}, t_{3}$ for (1) (2) (3) are 1000, 3333, $4000 \mu \mathrm{s}$. 


\subsection{DEVELOPMENT OF A THEORETICAL MODEL}

Based on the FE observations in the previous section, an analytical model approximating dynamic failure of plates with large dimensions in the longitudinal direction is developed. First, an imaginary patch plate on a long plate is defined. Second, governing equations are established for the predefined patch plate on the long plate, and in-plane stress resultants of the patch plate are estimated from the theory of elastic and elastic-plastic stress wave propagation. Third, the out-of-plane displacement of the patch plate is calculated by using the Galerkin method until the patch plate fails. The reaction forces at both of the ends of the long plate and the out-of-plane displacement of the parch plate can be obtained from the proposed model.

\subsubsection{Patch Plate}

Let a patch plate be an imaginary rectangle on a long plate which encompasses an excessively growing wrinkle as shown in Fig. 4.5(a) and (b). It is assumed that the long plate simulates either a core plate or a face plate of corrugated core sandwich columns in compression parallel-to-corrugations. Accordingly, the size of the patch plate on the long plate corresponds to the buckling wavelength. This idea starts from the observation that a repetitive buckling pattern appears on a long plate in compression and then one of wrinkles grows to excessive deformation. Simply-supported condition is assumed on the loaded side of the patch while lateral side expansion and rotation on the lateral sides is assumed to be restrained as shown in Fig. 4.5(c).

The width of the patch plate, $b$, can be either $b=\ell$ or $2 \ell \cdot \cos \omega$ for core plates of sandwich columns or face plates, respectively. On the other hand, the longitudinal 

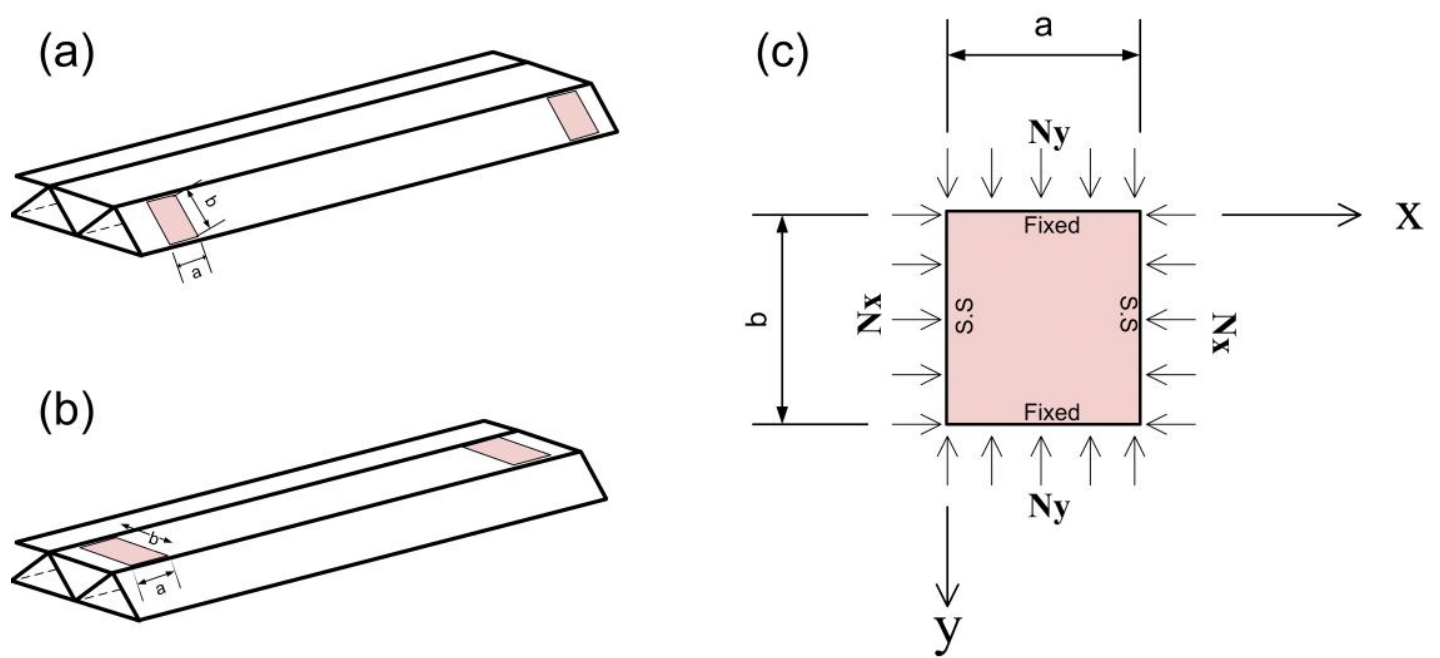

Figure 4.5: (a) Patch plates on a core plate of a corrugated core sandwich column: (b) Patch plates on a face plate of a corrugated core sandwich column; (c) Boundary conditions of the parch plates.

dimension of the patch plate, ' $a$ ' is determined from Eigen-buckling analysis minimizing in-plane force resultant, $\mathrm{N}_{\mathrm{x}}$. For example, that is defined to $\mathrm{be} \mathrm{a} / \mathrm{b}=0.78$ for elastic plates with Poisson's ratio of 0.3 , and $\mathrm{a} / \mathrm{b}=0.29$ for elastic-plastic plates having a plastic modulus of $\mathrm{E}_{\mathrm{p}}=534.2 \mathrm{MPa}$. The derivation is presented in Appendix $\mathrm{C}$ in conjunction with the governing equations in Section 4.3.2. It is assumed that the patch plates are placed near the ends. When the imaginary patch plates are placed near the ends, they are subjected to compressive loading, either with greater magnitude or for longer duration, than when they are placed in the other locations along the column length.

\subsubsection{Governing Equations}

The equation of motion for plates under in-plane forces $\mathrm{N}_{\mathrm{x}}$ and $\mathrm{N}_{\mathrm{y}}$ is $[81,82]$

$$
\left(\frac{\partial^{4} M_{x}}{\partial x^{4}}+2 \frac{\partial^{4} M_{x y}}{\partial x^{2} \partial y^{2}}+\frac{\partial^{4} M_{y}}{\partial y^{4}}\right)+N_{x} \cdot\left(\frac{\partial^{2} w}{\partial x^{2}}+\frac{\partial^{2} w_{0}}{\partial x^{2}}\right)+N_{y} \cdot\left(\frac{\partial^{2} w}{\partial y^{2}}+\frac{\partial^{2} w_{0}}{\partial y^{2}}\right)=\rho A \frac{\partial^{2} w}{\partial t^{2}}
$$


where $M_{x}, M_{x y}, M_{y}$ are the bending moments per unit length (stress couples), $N_{x}, N_{y}$ are in-plane stress resultants, $\rho$ is density, and $\mathrm{A}$ is plate thickness. The above equation (4.1) is independent of material properties [82].

Consider an elastic plate under bi-axial in-plane forces, and $\mathrm{N}_{\mathrm{x}}$ and $\mathrm{N}_{\mathrm{y}}$, with an initial curvature $\mathrm{w}_{0}(\mathrm{x}, \mathrm{y})$ in the out-of-plane direction. The kinematic assumptions in the Kirchhoff-Love hypothesis and the von Karman theory are applied as follows [82,83],

(i) Straight lines normal to the undeformed mid-plane of the plate remain straight and normal to the mid-plane after a deformation (Kirchhoff-Love).

(ii) The thickness of plate does not change during a deformation (Kirchhoff-Love).

(iii) Moderately large rotations in strain measure are allowed (von Karman).

The governing equation of elastic isotropic plates in the absence of shearing stress is derived as below [81]:

$$
D\left(\frac{\partial^{4} w}{\partial x^{4}}+2 \frac{\partial^{4} w}{\partial x^{2} \partial y^{2}}+\frac{\partial^{4} w}{\partial y^{4}}\right)+N_{x}\left(\frac{\partial^{2} w}{\partial x^{2}}+\frac{\partial^{2} w_{0}}{\partial x^{2}}\right)+N_{y}\left(\frac{\partial^{2} w}{\partial y^{2}}+\frac{\partial^{2} w_{0}}{\partial y^{2}}\right)=\rho A \frac{\partial^{2} w}{\partial t^{2}}
$$

In Eqn. (4.2), w is out-of-plane deflection, $\mathrm{D}$ is the flexural rigidity, $\mathrm{N}_{\mathrm{x}}$ and $\mathrm{N}_{\mathrm{y}}$ are the in-plane stress resultants on the loaded side and constrained side, respectively.

The governing equations used in inelastic plates are based on the work done by Bleich [84] and Becque [69], in which they focused on the quasi-static buckling strength of inelastic plates. Since the inelastic plate analysis has been argued up to the present, both Bleich's approach and Becque's are adopted and modified into dynamic versions.

Unlike elastic plates, governing equations for inelastic plates have not been established on a strict theoretical basis $[69,84-86]$. The source of the argument is that the plates subject to axial loading above the yield limit are no longer isotropic according to 
the theory of plasticity. Bleich's approach [84], called 'a semi-rational theory', has been known to be superior to others' [84-86] in that the experimental results are the bestcorrelated. Becque [69] attempted to discuss this issue on a more theoretical basis in his paper. The original forms of Bleich's and Becque's equation are given as below:

- Bleich's (Eqn. 4.3) and Becque's (Eqn. 4.4) equations

$$
\begin{aligned}
& D\left(\tau \frac{\partial^{4} w}{\partial x^{4}}+2 \sqrt{\tau} \frac{\partial^{4} w}{\partial x^{2} y^{2}}+\frac{\partial^{4} w}{\partial y^{4}}\right)+N_{x} \frac{\partial^{2} w}{\partial x^{2}}=0 \\
& \varphi \frac{t^{3}}{12}\left(E_{t} \frac{\partial^{4} w}{\partial x^{4}}+F \frac{\partial^{4} w}{\partial x^{2} y^{2}}+E \frac{\partial^{4} w}{\partial y^{4}}\right)+N_{x} \frac{\partial^{2} w}{\partial x^{2}}=0
\end{aligned}
$$

where

$$
\begin{aligned}
& \tau=\frac{E_{t}}{E}, \\
& F=\frac{E_{t}^{2}\left(\kappa^{2}-2 v \kappa+3 \kappa+6 v\right)+2 E_{t} E\left(-\kappa^{2}+v \kappa-\kappa+v+4\right)+E^{2}\left(\kappa^{2}-\kappa\right)}{(3+\kappa+4 v) E_{t}+(1-\kappa) E} \\
& \varphi=\frac{E}{(1+v \kappa) E-v(v+\kappa) E_{t}} .
\end{aligned}
$$

In the above equations, $\mathrm{E}, \mathrm{E}_{\mathrm{t}}, v$ denotes Young's modulus, tangent modulus under uniaxial loading and Poisson's ratio, respectively. The parameter $\kappa$ is defined as the ratio of principal plastic strain increments in uniaxial stress states, and determined from the following relationship:

$$
\dot{\varepsilon}_{P, 2}=\kappa \dot{\varepsilon}_{P, 1}
$$


If the von Mises stress yield criterion, f, is employed, Eqn. (4.5) can be transformed into Eqn. (4.6) and $\kappa$ becomes $-\frac{1}{2}$.

$$
\kappa=-\left.\frac{\partial f / \partial \sigma_{1}}{\partial f / \partial \sigma_{2}}\right|_{\sigma_{2}=0}=-\frac{1}{2}
$$

The dynamic versions of their quasi-static governing equations are derived as follows: As the first terms in Eqns. (4.3) and (4.4) correspond to the term $\left(\frac{\partial^{2} M_{x}}{\partial x^{2}}+\right.$ $\left.2 \frac{\partial^{2} M_{x y}}{\partial x \partial y}+\frac{\partial^{2} M_{y}}{\partial y^{2}}\right)$ in Eqn. (4.1), the dynamic versions of the inelastic governing equations are obtained by modifying Eqns. (4.3) and (4.4).

$$
\begin{aligned}
& D\left(\tau \frac{\partial^{4} w}{\partial x^{4}}+2 \sqrt{\tau} \frac{\partial^{4} w}{\partial x^{2} y^{2}}+\frac{\partial^{4} w}{\partial y^{4}}\right)+N_{x}\left(\frac{\partial^{2} w}{\partial x^{2}}+\frac{\partial^{2} w_{0}}{\partial x^{2}}\right)+N_{y}\left(\frac{\partial^{2} w}{\partial y^{2}}+\frac{\partial^{2} w_{0}}{\partial y^{2}}\right)=\rho A \frac{\partial^{2} w}{\partial t^{2}} \\
& \varphi \frac{t^{3}}{12}\left(E_{t} \frac{\partial^{4} w}{\partial x^{4}}+F \frac{\partial^{4} w}{\partial x^{2} y^{2}}+E \frac{\partial^{4} w}{\partial y^{4}}\right)+N_{x}\left(\frac{\partial^{2} w}{\partial x^{2}}+\frac{\partial^{2} w_{0}}{\partial x^{2}}\right)+N_{y}\left(\frac{\partial^{2} w}{\partial y^{2}}+\frac{\partial^{2} w_{0}}{\partial y^{2}}\right)=\rho A \frac{\partial^{2} w}{\partial t^{2}}
\end{aligned}
$$

Therefore, Eqn. (4.2) for elastic plates and Eqns. (4.7) and (4.8) for elastic-plastic plates will be used as the governing equations of imaginary patch plates.

\subsubsection{Galerkin Method and Estimation of In-plane forces}

The governing equations in the previous section are solved by employing the Galerkin technique. Considering the boundary conditions on the sides, the out-of-plane displacement field, $w(x, y, t)$, initial curvatures, $w_{0}(x, y)$ and virtual displacement, $\delta w(x, y)$ are given by:

$$
w(x, y, t)=T(t) \cdot X(x) \cdot Y(y)=T(t) \cdot \sin \left(\frac{\pi x}{a}\right) \cdot \frac{1}{2}\left(1-\cos \left(\frac{2 \pi y}{b}\right)\right)
$$




$$
\begin{aligned}
& w_{0}(x, y)=\xi_{0}^{(L P)} \cdot X(x) \cdot Y(y)=\xi_{0}^{(L P)} \cdot \sin \left(\frac{\pi x}{a}\right) \cdot \frac{1}{2}\left(1-\cos \left(\frac{2 \pi y}{b}\right)\right) \\
& \delta w(x, y)=\delta T \cdot X(x) \cdot Y(y)=\delta T \cdot \sin \left(\frac{\pi x}{a}\right) \cdot \frac{1}{2}\left(1-\cos \left(\frac{2 \pi y}{b}\right)\right)
\end{aligned}
$$

In Eqn.(4.9), $\mathrm{T}(\mathrm{t})$ is a modal amplitude, which represents the out-of-plane displacement at the midpoint of a patch plate, and $\xi_{0}^{(L P)}$ is the magnitude of an initial curvature. Applying Galerkin method to the elastic governing equation (4.2),

$$
\begin{array}{r}
\int_{0}^{b} \int_{0}^{a} \nabla^{4} w \cdot \delta w d x d y+\frac{N_{x}}{D} \int_{0}^{b} \int_{0}^{a} \frac{\partial^{2}\left(w+w_{0}\right)}{\partial x^{2}} \bullet \delta w d x d y+\frac{N_{y}}{D} \int_{0}^{b} \int_{0}^{a} \frac{\partial^{2}\left(w+w_{0}\right)}{\partial y^{2}} \cdot \delta w d x d y \\
=\frac{\rho A}{D} \int_{0}^{b} \int_{0}^{a} \frac{\partial^{2} w}{\partial t^{2}} \cdot \delta w d x d y
\end{array}
$$

In Eqn. (4.10), the in-plane resultant $\mathrm{N}_{x}(t)$ for the patch plate is estimated from the in-plane stress distribution of the long plate compressed at a constant velocity by applying the theory of elastic stress wave propagation. (See Section 3.3.1. to estimate $\mathrm{N}_{x}(t)$, just the superscript (perp) needs to be replaced by (para)). On the other hand, $N_{y}(t)$ is obtained from the relationship of $N_{y}=v \mathrm{~N}_{x}$ considering the constraint of lateral expansion. Substituting Eqns. (4.9a),(4.9b),(4.9c) into Eqn. (4.10), a second order ordinary differential equation can be obtained. The equation is solved until the conditions that the extensional rate of the mid plane of patch plate is greater than the compression rate are satisfied.

$$
\begin{gathered}
\int_{0}^{a} \int_{0}^{b} \overline{\dot{\varepsilon}}_{\mathrm{ex} t} d x \cdot d y=\int_{0}^{a} \int_{0}^{b} \overline{\dot{\varepsilon}}_{\text {comp }} d x \cdot d y \\
\text { where } \quad \int_{0}^{b} \int_{0}^{a} \overline{\dot{\varepsilon}}_{\text {comp }} d x \cdot d y \approx \int_{0}^{b} \int_{0}^{a}\left(\frac{V}{L}\right) d x \cdot d y=\frac{V a b}{L}
\end{gathered}
$$




$$
\int_{0}^{b} \int_{0}^{a} \overline{\dot{\varepsilon}}_{\mathrm{ext}} d x \cdot d y \approx\left(\frac{3 \pi^{2}}{16}\right)\left(\frac{b}{a}\right) T \dot{T}+\left(\frac{3 \pi^{2}}{16}\right)\left(\frac{b}{a}\right) \xi_{0}^{(L P)} \dot{T}
$$

Likewise, the two governing equations for inelastic plates, Eqns. (4.7) and (4.8) are rewritten as an integral form as seen in Eqns. (4.12) and (4.13).

$$
\begin{aligned}
& \int_{0}^{b} \int_{0}^{a}\left[D\left(\tau \frac{\partial^{4} w}{\partial x^{4}}+2 \sqrt{\tau} \frac{\partial^{4} w}{\partial x^{2} y^{2}}+\frac{\partial^{4} w}{\partial y^{4}}\right)+N_{x} \frac{\partial^{2}\left(w+w_{0}\right)}{\partial x^{2}}+N_{y} \frac{\partial^{2}\left(w+w_{0}\right)}{\partial y^{2}}\right] \cdot \delta w d x d y \\
& =\frac{\rho A}{D} \int_{0}^{b} \int_{0}^{a} \frac{\partial^{2} w}{\partial t^{2}} \bullet \delta w d x d y \\
& \int_{0}^{b} \int_{0}^{a}\left[\varphi \frac{t^{3}}{12}\left(E_{t} \frac{\partial^{4} w}{\partial x^{4}}+F \frac{\partial^{4} w}{\partial x^{2} y^{2}}+E \frac{\partial^{4} w}{\partial y^{4}}\right)+N_{x} \frac{\partial^{2}\left(w+w_{0}\right)}{\partial x^{2}}+N_{y} \frac{\partial^{2}\left(w+w_{0}\right)}{\partial y^{2}}\right] \cdot \delta w d x d y \\
& =\frac{\rho A}{D} \int_{0}^{b} \int_{0}^{a} \frac{\partial^{2} w}{\partial t^{2}} \cdot \delta w d x d y
\end{aligned}
$$

As a result, the governing equations, Eqns (4.12) and (4.13) for inelastic plates, can be reduced to second order ODEs with respect to time by substituting the assumed displacement field of Eqn. (4.9) and applying the Galerkin method. In a similar manner to the elastic plate analysis, $\mathrm{N}_{x}(t)$ is estimated from the theory of elastic-plastic wave propagation. (See Section 3.3.1. To estimate $\mathrm{N}_{x}(t)$ here, just the superscript (perp) needs to be replaced by (para)). Here, $N_{y}(t)$ is obtained from the relationship of $N_{y}=v_{P} \mathrm{~N}_{x}$ considering the restriction of lateral expansion. The ODEs are solved until the load drop criterion is satisfied as described in Eqn. (4.11). Note that at the transition from elastic to elastic-plastic material state, the size of the patch plate, ' $a$ ', is redefined according to Appendix $\mathrm{C}$. In addition, the value of $\mathrm{T}(\mathrm{t})$ at the end of elastic plate analysis (i.e., as soon as the plate material yields) is assumed to be used for the initial value of $\mathrm{T}(\mathrm{t})$ in the elastic-plastic plate analysis. 


\subsection{RESULTS AND DISCUSSION}

\subsubsection{FE Validation for Plate buckling}

In order to check the validity of the proposed theoretical approach, Finite Element Analysis (FEA) of plates with a long dimension is performed. The parent material of the plates is thought to be either A16061-T6 or SS304 to apply the theoretical model to a general metallic material for corrugated core sandwich columns. In particular, three kinds of features are validated through the FEA: a) the size of patch, and b) comparison of reaction force from model and simulation and c) the mid-point displacements of patch plates (i.e., modal amplitude). For construction of the FE models, material properties of the parent materials, A16061-T6 and SS304, are modeled as elaborated in Chapters 2, and conventional shell elements with reduced integration (S4R) are meshed on the plates. Boundary conditions applied in the FE models are described in Fig. 4.6: All degrees of freedom are fixed at the back end, while all DOFs except for the translational DOF in the $\mathrm{x}$-direction are fixed on the axially loaded side. On the lateral sides of plates, lateral expansion and rotations are constrained considering the constraining effect of neighboring materials in sandwich columns. To include geometric curvature imperfections, the first eigen-mode with a magnitude $\xi^{(\mathrm{LP})}$ is superimposed to the $\mathrm{FE}$ model of perfectly flat geometry. The eigenshape with $\xi^{(\mathrm{LP})}=1$ as shown in Fig. 4.7 reveals a repetitive buckling pattern, however, the amplitude of each wrinkle is different, but of the same order.

For validation, FE simulations are performed on two Al6061-T6 plates and two SS304 plates with different thicknesses, respectively: the thinner plate fails in the elastic 


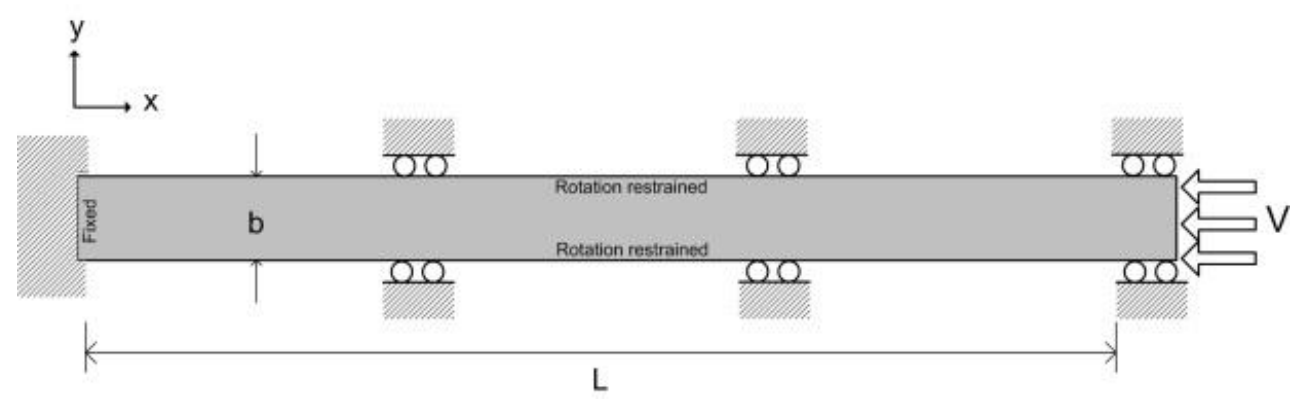

Figure 4.6: Boundary conditions of FEA of plates.

region while the thicker one does in the plastic region. The plates are subjected to $\mathrm{V}=1 \mathrm{~m} / \mathrm{s}$.

1) Elastic plates ; Two cases are presented for validation of the elastic plate analytical model. One is an Al6061-T6 plate of $\mathrm{W}=22.0 \mathrm{~mm}, \mathrm{~L}=300 \mathrm{~mm}$, and thickness $\mathrm{A}=0.457 \mathrm{~mm}$ with an imperfection magnitude $\xi^{(\mathrm{LP})}=0.001$ under $\mathrm{V}=1 \mathrm{~m} / \mathrm{s}$. The other one is an SS304 plate of $\mathrm{W}=35.35 \mathrm{~mm}, \mathrm{~L}=353 \mathrm{~mm}$, and $\mathrm{A}=0.25 \mathrm{~mm}$ with $\xi^{(\mathrm{LP})}=0.001$ under V=1m/s. Figs. 4.8(a) and 4.9(a) show the reaction force histories and out-ofplane displacements at the midpoint of the patch plates (or the displacements of the failed wrinkles in the FEM) for the two elastic cases, respectively. The times-tofailure in the two cases are predicted by the analytical model accurately. The out-ofplane displacements at the mid-point of the failed wrinkles (modal amplitude from the analytical model) are in good agreement with the FE simulations.

From Figs. 4.8(b)(c) and 4.9(b)(c), the sizes of the wrinkles leading to failure are measured from the FE simulations. The excessively deformed wrinkles are located in the middle of plates unlike the basic assumption about the locations of the patch plates in the analytical approach. This is to be expected as the initial curvature 


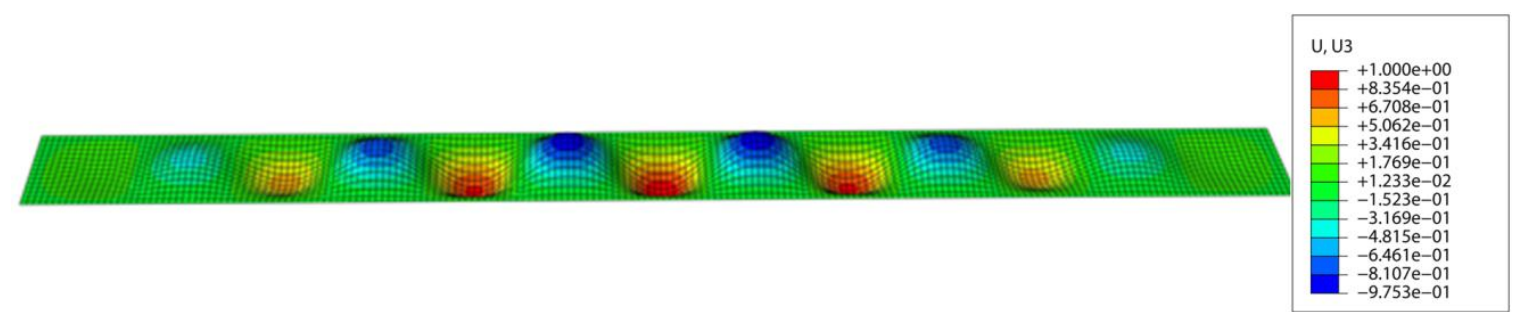

Figure 4.7: The assigned eigenmode shape superposed for initial curvature imperfections. A repetitive buckling pattern is shown, however, the amplitude of each wrinkle is different, but of the same order. In dynamic analysis of plates, the mode shape with a magnitude $\xi$ is added to the flat plates.

imperfections assigned in the $\mathrm{FE}$ simulations have repetitive wrinkles with the different amplitudes as shown in Fig. 4.7.

The contours plots for the out-of-plane displacement field, shown in Figs. 4.8(b)(c) and $4.9(b)(c)$ demonstrate that $\mathrm{x}=\mathrm{a} / \mathrm{b} \sim 0.7$, which agrees well with the kinematic assumption of the predetermined patch size ( $\mathrm{x} \sim 0.78$ and 0.77 for the Al6061-T6 (Eqn. (C6a)) and SS304 elastic plates (Eqn. (C7a)), respectively) as given in the analysis in Appendix C. 


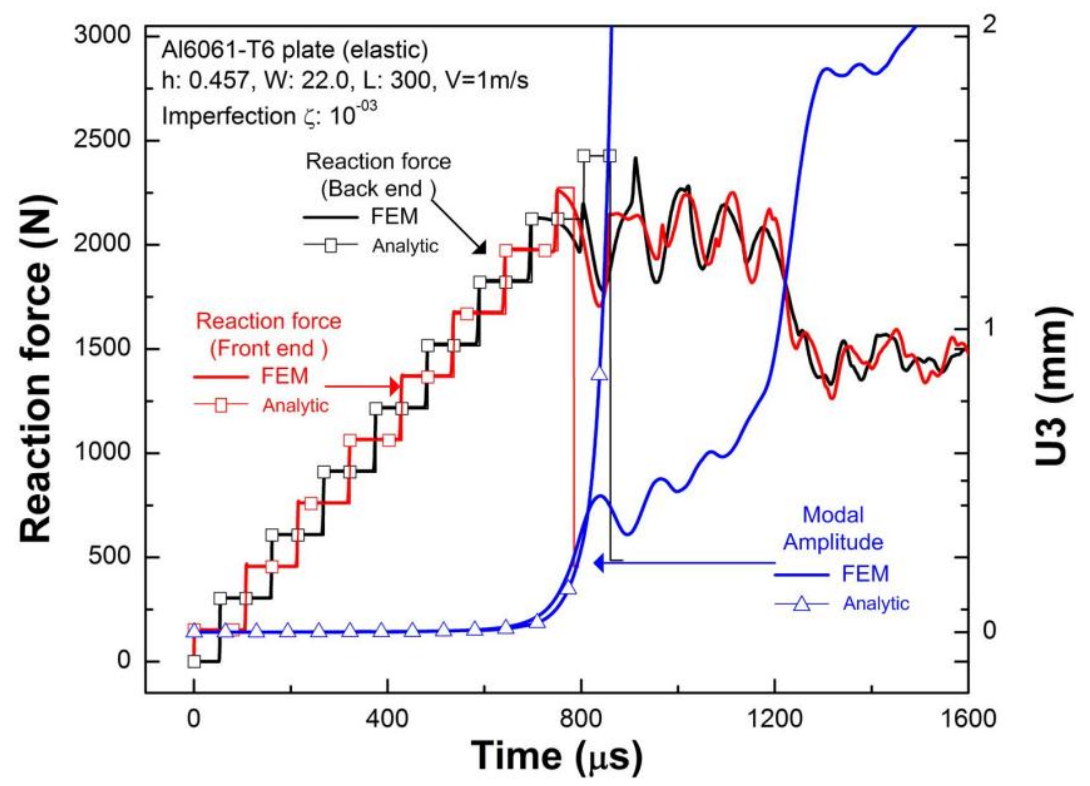

(a)

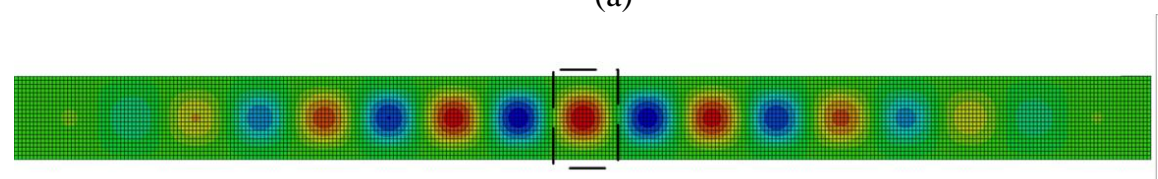

(b) $t=600 \mu \mathrm{s}$

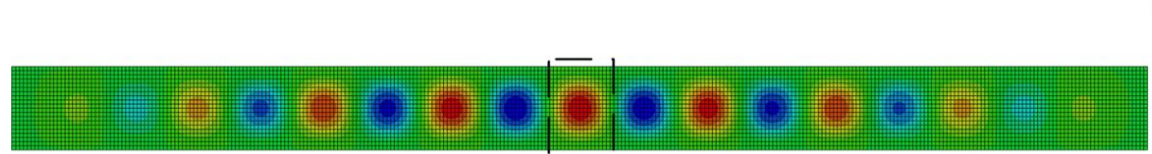

(c) $\bar{t}=800 \mu \mathrm{s}$

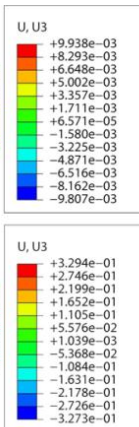

Figure 4.8 : FE validation of the analytical model for the Al6061-T6 elastic plate (h:0.457mm, W: $22.0 \mathrm{~mm}, \mathrm{~L}: 300 \mathrm{~mm}, \xi=0.001, \mathrm{~V}=1 \mathrm{~m} / \mathrm{s}$ ): (a) Reaction force and out-of-plane displacement at the midpoint of the patch plate (or failed wrinkle); (b)\&(c) out-of-plane displacement at $\mathrm{t}=600$ and $800 \mu \mathrm{s}$, respectively. 


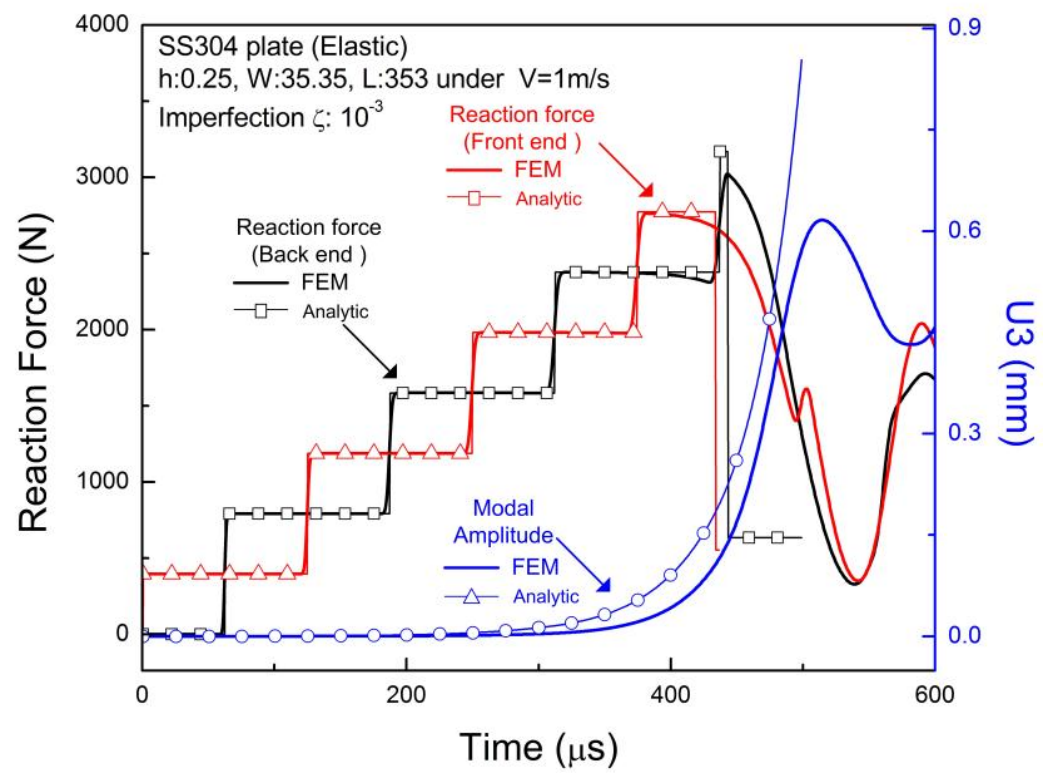

(a)

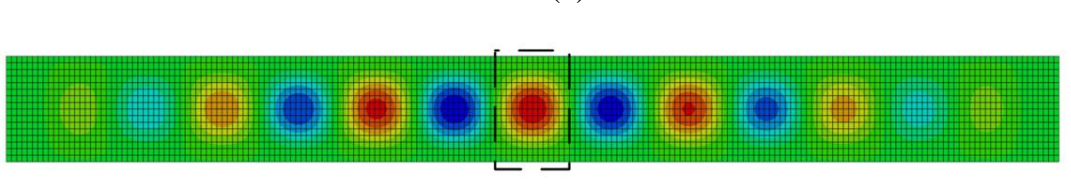

(b) $t=300 \mu \mathrm{s}$

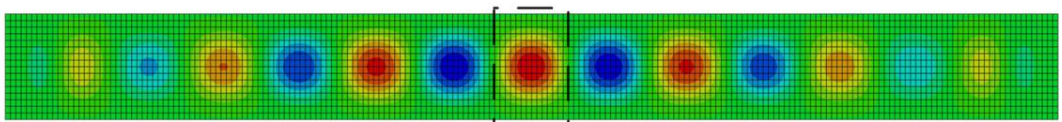

(c) $\mathrm{t}=400 \mu \mathrm{s}$

Figure 4.9 : FE validation of the analytical model for the SS304 elastic plate (h:0.25mm, W: 35.35mm, $\mathrm{L}: 353 \mathrm{~mm}, \xi=0.001, \mathrm{~V}=1 \mathrm{~m} / \mathrm{s}$ ): (a) Reaction force and out-of-plane displacement at the midpoint of the patch plate (or failed wrinkle); (b)\&(c) out-of-plane displacement at $\mathrm{t}=300$ and $400 \mu \mathrm{s}$, respectively. 
2) Inelastic plates; Two cases are presented for validation of the inelastic plate analytical model. One is composed of A16061-T6 with $\mathrm{W}=22.0 \mathrm{~mm}, \mathrm{~L}=300 \mathrm{~mm}$, and $\mathrm{A}=0.88 \mathrm{~mm}$ with $\xi^{(\mathrm{LP})}=0.001$ where the front end is displaced at $\mathrm{V}=1 \mathrm{~m} / \mathrm{s}$. The other one is a SS304 plate with $\mathrm{W}=35.35 \mathrm{~mm}, \mathrm{~L}=353 \mathrm{~mm}$, and $\mathrm{A}=1.25 \mathrm{~mm}$ with $\xi^{(\mathrm{LP})}=0.001$ with $\mathrm{V}=1 \mathrm{~m} / \mathrm{s}$. Figs. 4.10(a) and 4.11(a) show the reaction force versus time histories and out-of-plane displacements at the midpoint of the patch plates (or the failed wrinkles in the FEM) for the two inelastic cases, respectively.

In Fig. 4.10, the analytical results employing Bleich's and Becque's governing equations for the Al6061-T6 plate predict more conservative results compared with the FEM results. Specifically, the duration in plastic deformation $(1100<\mathrm{t}<1450 \mu \mathrm{s})$ calculated by the FE simulation is much longer than that obtained from the analytical models. In Fig. 4.10(b)(c), the out-of-plane displacement fields obtained from the FE simulation are plotted during plastic deformation, especially at $\mathrm{t}=1267$ $\mu$ s and $1467 \mu \mathrm{s}$. At $\mathrm{t}=1267 \mu \mathrm{s}$, the buckling wave length is as large as that of an elastic plate $(\mathrm{x}=\mathrm{a} / \mathrm{b} \sim 0.7)$. However, at $\mathrm{t}=1467 \mu \mathrm{s}$, the critical buckle leading to the failure of the long plate has a much shorter wave length $(\mathrm{x}=\mathrm{a} / \mathrm{b} \sim 0.3)$ than the one observed at $\mathrm{t}=1267 \mu \mathrm{s}$. That is, the initial assumption that the critical buckling wave lengths in elastic-plastic plates (the size of elastic-plastic patch plate) are smaller than those in elastic plates (the size of elastic patch plate) is qualitatively confirmed. But, as shown in the FE result at $\mathrm{t}=1267 \mu$ s, the buckling wavelength does not change suddenly at the transition from elastic to plastic. Rather, the switch toward the shorter wave length seems to be gradual. It can be concluded that the discrepancy between the analytical models and the FEM can be accounted for by the 
gradual change in the patch size at the transition from elastic to plastic. As a result, the growth of the modal amplitude in the analytical models is much faster than the growth of out-of-plane displacement at the midpoint of the critical buckle in FEM. Therefore, earlier time-to-failure is predicted compared with the FEM.

Likewise, the SS304 plate show similar phenomena and conservative results are predicted compared with the FEM, which is presumably due to the gradual change toward the preferred buckling wave $(x \sim 0.3)$ in the plastic plate, which is shorter than that of an elastic plate (x 0.7), as shown in Fig. 4.11. 


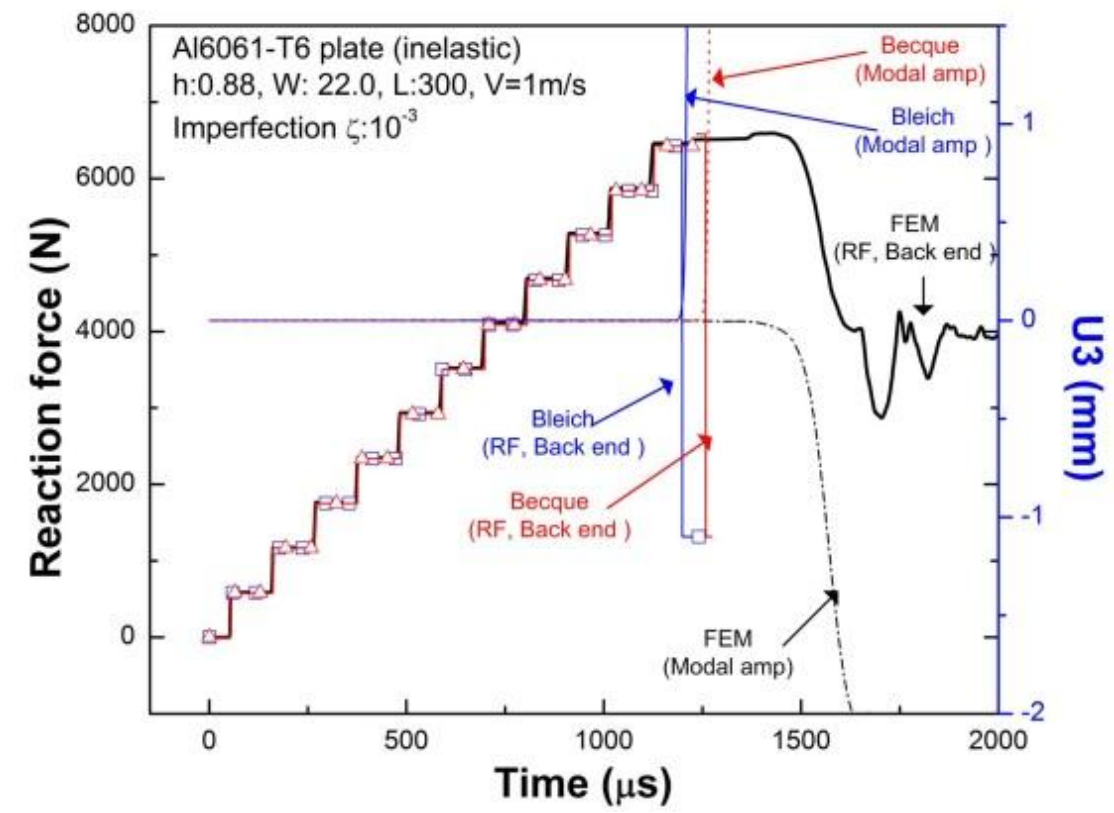

(a)

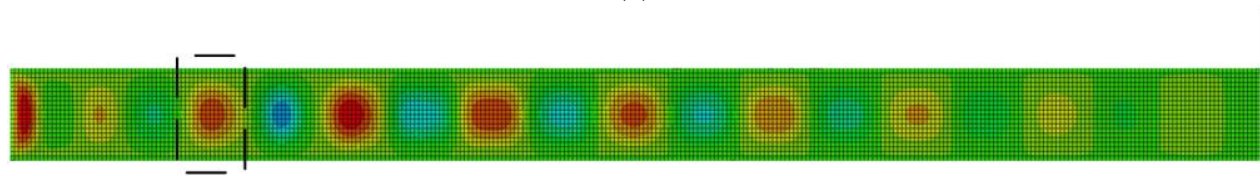

(b) $\mathrm{t}=1267 \mu \mathrm{s}$

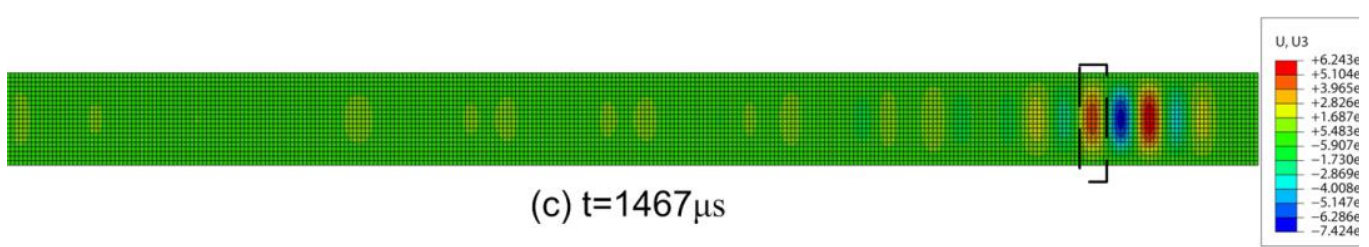

Figure 4.10 : FE validation of the analytic model for the Al6061 elastic-plastic plate (h:0.88, W: 22.0, $\mathrm{L}: 300, \zeta=0.001, \mathrm{~V}=1 \mathrm{~m} / \mathrm{s}$ ): (a) Reaction force and out-of-plane displacement at the midpoint of the patch plate (or failed wrinkle); (b)(c) out-of-plane displacement at $\mathrm{t}=1267$ and $1467 \mu \mathrm{s}$, respectively. 


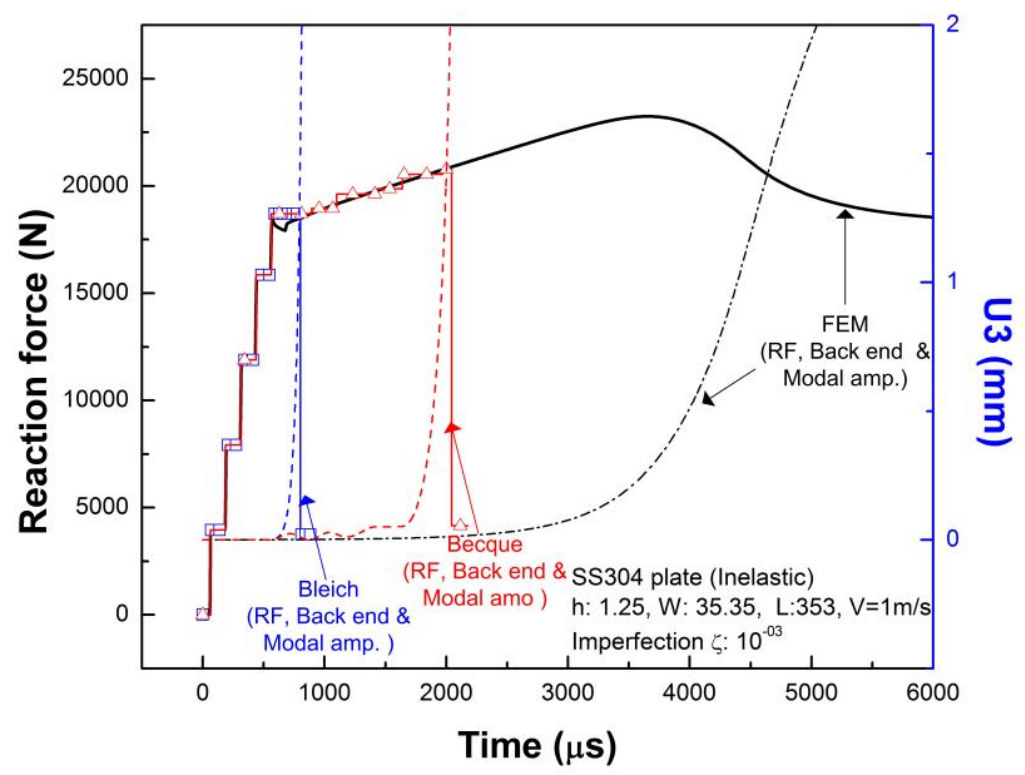

(a)

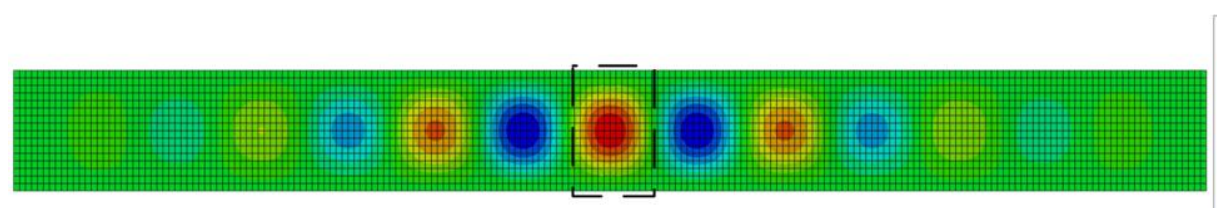

(b) $t=3200 \mu \mathrm{s}$
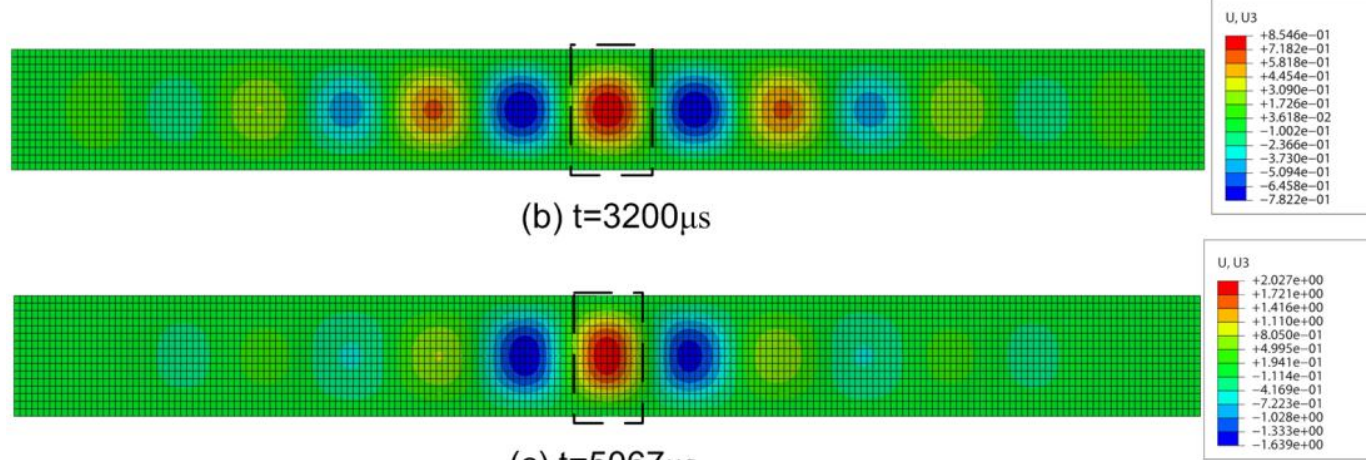

(c) $\mathrm{t}=5067 \mu \mathrm{s}$

Figure 4.11 : FE validation of the analytic model for the SS304 elastic-plastic plate (h:1.25, W: 35.35, $\mathrm{L}: 353, \zeta=0.001, \mathrm{~V}=1 \mathrm{~m} / \mathrm{s}$ ): (a) Reaction force and out-of-plane displacement at the midpoint of the patch plate (or failed wrinkle); (b)(c) out-of-plane displacement at $\mathrm{t}=3200$ and $5067 \mu$ s, respectively. 


\subsubsection{Discussion and Limitations}

As presented in the previous section 4.4.1, the proposed analytical model predicts the dynamic response of elastic plates at $\mathrm{V}=1 \mathrm{~m} / \mathrm{s}$ with good accuracy while the time-toload drop prediction by the elastic-plastic plate analytical model is premature.

This section discusses the sources of discrepancy observed in (a) the elastic-plastic plate model and (b) postcritical behavior in elastic plates compressed at a velocity of $\mathrm{V}=0.1 \mathrm{~m} / \mathrm{s}$ lower than $\mathrm{V}=1 \mathrm{~m} / \mathrm{s}$ (The plate response at the lower velocity of $\mathrm{V}=0.1 \mathrm{~m} / \mathrm{s}$ is included additionally in this section).

- A source of inaccuracy in the elastic-plastic plate model.

A source leading to the inaccurate analytical predictions, especially in the elasticplastic plate analysis, is found in the definition of the size of elastic-plastic patch plates (or equivalently, buckling wave length). In the development of the analytical model, the size of patch plate is predefined depending on its material properties and plate width. Unlike the buckling wavelength in face wrinkling failure mode (the distance between nodes), the plate buckling wavelength has a continuous distribution in nature.

In elastic plates having initial curvature imperfections, the out-of-plane displacements oscillate, while retaining their modal structures and amplify the original imperfection magnitudes [30]. Thus, a reasonable approximation is expected for the dynamic response of elastic plates if geometric curvature imperfections are defined as accurately as in the FE analyses mathematically.

Inelastic plates are assumed to have a much smaller preferred buckling wavelength in the analytical model. As observed in the FE simulations, the size of the patch plate 
(buckling wavelength) changes gradually over time rather than suddenly at their material state transition from elastic to plastic region. The gradual change in the buckling wavelength during plastic deformation is a source of the conservative predictions.

- A discrepancy due to the postcritical behavior of elastic plates at a lower velocity of $\mathrm{V}=0.1 \mathrm{~m} / \mathrm{s}$ than $\mathrm{V}=1 \mathrm{~m} / \mathrm{s}$.

According to the theory of plates, elastic plate elements under quasi-static in-plane loading are generally known to have a capacity to support additional loads without a load drop beyond their critical strength because of their postcritical reserve $[64,82]$. The postcritical reserve of plates [64] is known to result from the redistribution of the inplane force resultants, and characterized by a reduced stiffness in a load-displacement curve.

However, the dynamic response of the elastic plates at $\mathrm{V}=1 \mathrm{~m} / \mathrm{s}$ presented in the previous section 4.4.1 does not show such phenomena. Instead, such features are discovered in elastic plates under a lower applied velocity, in this case when $V=0.1 \mathrm{~m} / \mathrm{s}$. The FE simulations and analytical predictions for $\mathrm{V}=0.1 \mathrm{~m} / \mathrm{s}$ will be presented additionally in this section.

Consider two Al6061-T6 plates at V=0.1m/s of length $\mathrm{L}=300 \mathrm{~mm}, \mathrm{~W}=22.0 \mathrm{~mm}$, and the plate thicknesses $A=0.1 \mathrm{~mm}$ and $0.457 \mathrm{~mm}$, respectively. Additional FEA and analytic predictions are performed for the low velocity cases and the results are shown in Fig. 4.12. The FE results show a change in stiffness over time unlike the sudden load drop shown previous in Figs. 4.8 and 4.9. Although the proposed analytical model successfully predicts the linearly-increasing region, the postcritical behavior characterized by stiffness 
reduction $(\mathrm{t}>1000 \mu \mathrm{s}$ for $\mathrm{A}=0.1 \mathrm{~mm}$ and $\mathrm{t}: 6000 \sim 10500 \mu \mathrm{s}$ for $\mathrm{A}=0.457 \mathrm{~mm}$, respectively) cannot be described.

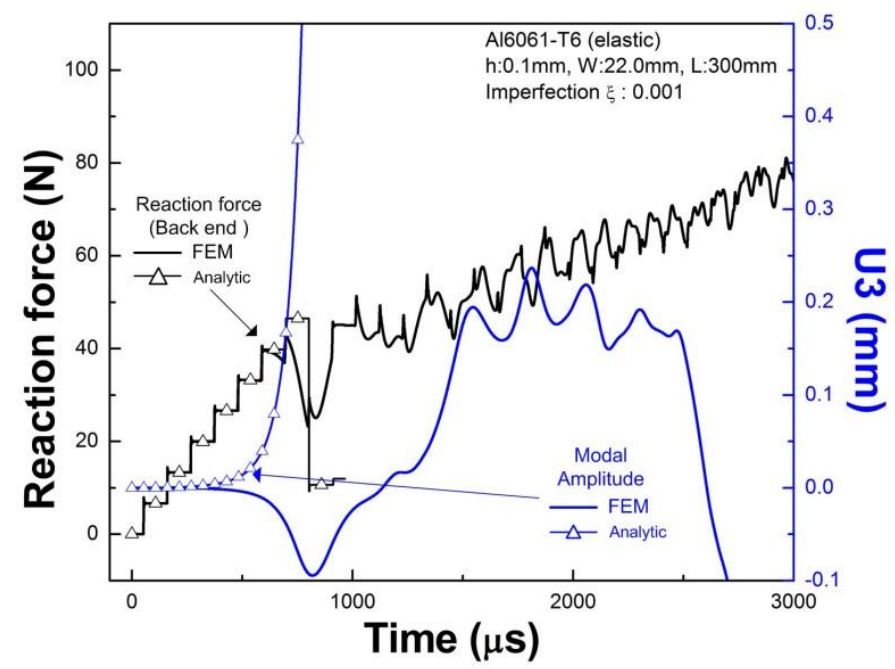

(a)

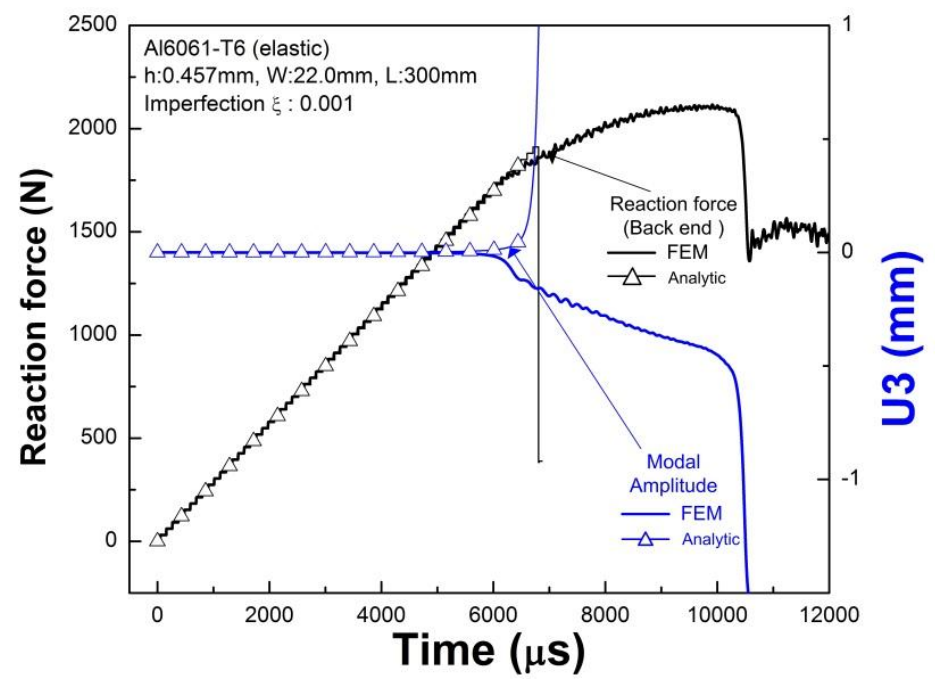

(b)

Figure 4.12: Post critical responses of the Al6061-T6 elastic plates of two different plate thicknesses under $\mathrm{V}=0.1 \mathrm{~m} / \mathrm{s}$ calculated from FEM and the analytical model: (a) $\mathrm{h}=0.1 \mathrm{~mm}$; (b) $\mathrm{h}=0.457 \mathrm{~mm}$. 
To sum up, the proposed model has two limitations regarding this observation: First, it is not capable of predicting the response for the stiffness-reduction region. Second, it is not able to predict whether such response, which is depending on the applied velocities, appears or not. Whether the region of stiffness reduction is included for a design or not depends on designers' choice. Sometimes, it may become too conservative if the region is ignored [64].

\subsection{CHAPTER SUMMARY}

An attempt to approximate the local plate buckling response of sandwich columns dynamically compressed parallel-to-corrugations was made through developing an analytical model for long plates. The analytical model is based on the assumption that the structural integrity of the sandwich columns is determined by the occurrence and growth of a buckle in imaginary patch plates along the length of the column in the face and the core.

The analytical prediction for the elastic plate response at $\mathrm{V}=1 \mathrm{~m} / \mathrm{s}$ was wellcorrelated with the FE calculations as presented in Section 4.4.1. The elastic plates at $\mathrm{V}=1 \mathrm{~m} / \mathrm{s}$ show sudden load drops when they reach critical state. However, at a lower velocity of $V=0.1 \mathrm{~m} / \mathrm{s}$ (additionally presented in Section 4.4.2), the post critical response characterized by a reduced stiffness could not be captured, which is usually seen in plate buckling under quasi-static loading [64].

In the case of elastic-plastic plate buckling, two governing equations were employed. However, the elastic-plastic plate buckling response was not successfully 
predicted. The discrepancy is thought to result from the inaccurate definition of elasticplastic buckling wavelength (the size of imaginary patch plate).

As a cautious suggestion, if an imaginary patch plate which encompasses either a continuous distribution of buckling wavelengths or many discrete buckling wavelengths is defined, the inelastic results could be improved in the future. However, it may be more practical to use the model combined with FE simulations of individual plates by establishing its applicable range by FEM. (Local plate buckling failure is easily seen in the corrugated core sandwich columns employing thin plates at a relatively high velocity, as observed in Section 4.2.)

Despite these drawbacks, the work throughout this chapter will be meaningful on the conditions that

1) Manufacturing imperfections are well-defined,

2) Deformation is within elastic range,

3) Applied velocity is over $1 \mathrm{~m} / \mathrm{s}$.

4) Membrane force is not significant (i.e., the plate width-to-thickness ratio, b/A, is not large). 


\section{CHAPTER 5}

\section{DYNAMIC FAILURE MECHANISM MAPS}

\subsection{INTRODUCTION}

In the design of sandwich structures, failure mechanism maps are useful tools for identifying the operative collapse mode by providing a visual representation $[28,87]$. For example, Biagi [27, 28, 103] constructed failure mechanism maps of corrugated core sandwich columns based on a quasi-static analysis, in which the effects of sandwich nondimensional parameters such as $t / \ell, \omega, h / \ell, L / \ell$ are investigated. The boundaries on the failure maps are expected to change if the rate-of-compression is increased and so is the focus for this current study.

In this chapter, dynamic effects on failure maps for corrugated core sandwich columns compressed perpendicular-to-corrugations will be investigated. To this end, two quasi-static failure mechanism maps of Al6061-T6 and SS304 corrugated core sandwich columns will be chosen for the dynamic investigation. The influences of rate-of-loading and geometric curvature imperfection are then analyzed for the selected failure maps. Dynamic response for individual failure modes will be obtained from the semi-analytical approaches for face wrinkling and global buckling developed from the previous chapters 2 and 3. 


\subsection{QUASI-STATIC FAILURE MECHANISM MAPS}

In this section, two quasi-static failure maps are used as the starting point for the analysis of dynamic effects on the failure boundaries. Figs. 5.1 and 5.2 describe the quasi-static failure maps of Al6061-T6 and SS304 corrugated core sandwich columns and the specifications of core dimensions.

Quasi-static failure maps of corrugated core sandwich columns, with axes of $L / \ell$

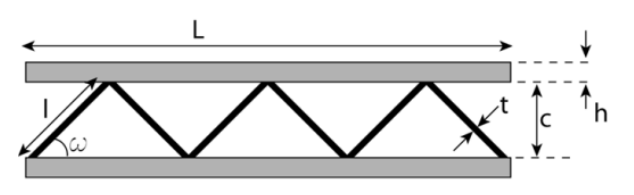

$$
\begin{aligned}
& t=3.175(\mathrm{~mm}) \\
& \mathrm{l}=22.0(\mathrm{~mm}) \\
& \omega=60^{\circ}
\end{aligned}
$$

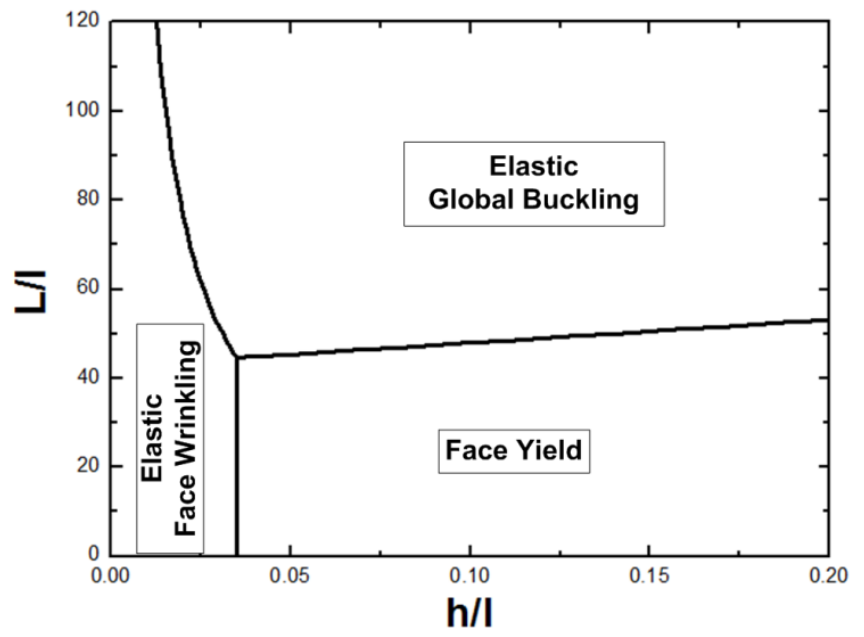

Figure 5.1: Target core geometry of Al6061-T6 corrugated core sandwich columns and corresponding quasi-static failure map.

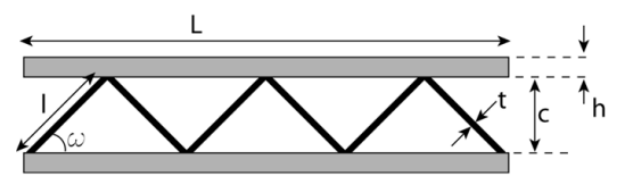

$\mathrm{t}=1.5(\mathrm{~mm})$

$\mathrm{I}=25.0(\mathrm{~mm})$

$\omega=45^{\circ}$

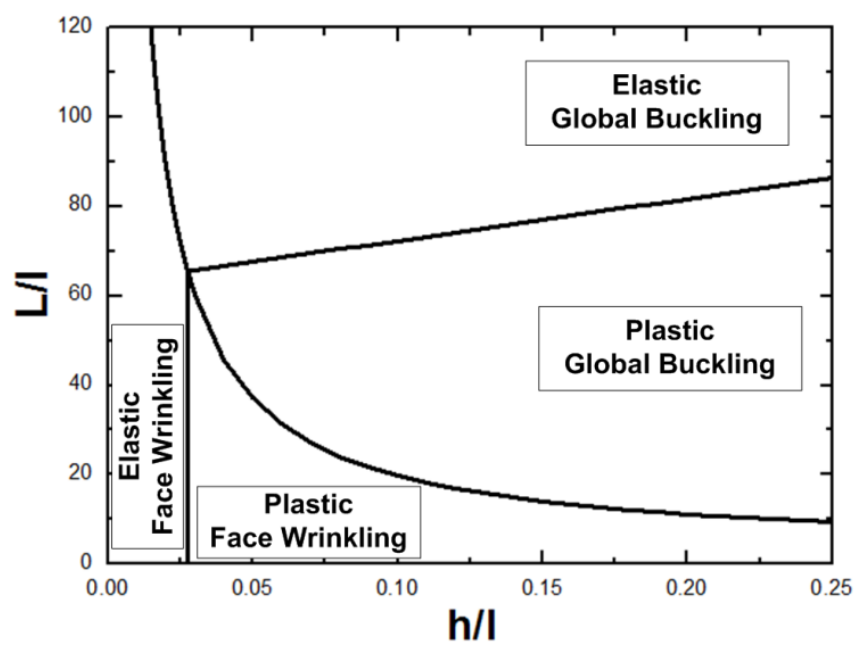

Figure 5.2: Target core geometry of SS304 corrugated core sandwich columns and corresponding quasistatic failure map. 
and $h / \ell$, have been presented by Biagi [27, 28, 103]. Failure maps can be constructed for different core geometric parameters, $t / \ell$, and $w$. However, the dynamic investigation will be limited to the two quasi-static failure maps regarding the cores, which have been used for the experimental validation carried out by Biagi et al. [27, 28]. Consequently, the target core dimensions for the dynamic investigation are as follows. One is an A16061-T6 corrugated core of corrugation thickness, $\mathrm{t}=3.175 \mathrm{~mm}$, corrugation length $\ell=22.0 \mathrm{~mm}$, corrugation angle, $\mathrm{w}=60^{\circ}$ having a relative core density of $25 \%$, and the other is a SS304 corrugated core of $\mathrm{t}=1.5 \mathrm{~mm}, \ell=25 \mathrm{~mm}, \mathrm{w}=45^{\circ}$ having a relative core density of $12 \%$. Material properties of the parent materials are used as presented in Section 2.5: Al6061-T6 has E=75150(MPa), $\sigma_{\mathrm{Y}}=293.9(\mathrm{MPa})$ and $E_{P}=\frac{d \sigma_{e q}}{d p}=$ 534.2(MPa). And, SS304 has $\mathrm{E}=230769.2(\mathrm{MPa}), \sigma_{Y}^{(0)}=271.8(M P a)$ and $E_{P}^{(1)}=$ $\frac{d \sigma_{e q}}{d p}=8117.65(M P a)$. The other hardening region for larger plastic strain $(\mathrm{p}>0.016)$ is not considered here.

Metallic corrugated core sandwich columns loaded quasi-statically perpendicularto-corrugations fail by one of these failure modes: elastic global buckling (EGB), plastic global buckling (PGB), elastic face wrinkling (EFW), plastic face wrinkling (PFW), elastic shear buckling [27, 28, 103]. This is the case for SS304 corrugated core sandwich columns. As columns manufactured from Al6061-T6 does not undergo much post yield hardening deformation under quasi-static loading [28], the number of failure modes in the failure map can be reduced to three (Fig. 5.1): elastic global buckling (EGB), elastic face wrinkling (EFW), face yield (FY).

To create failure maps, the analytical predictions of the collapse strength for the individual failure modes are employed [27, 28, 103]. By evaluating minimum strength 
among the possible failure modes, the dominant failure mode can be determined for a given geometry. Thus, sandwich columns corresponding to geometric points on the boundary are expected to fail by simultaneous activation of the adjacent failure modes. Refer to Biagi's study [27, 28, 103] for more details on the analytical expressions. The equations associated with quasi-static loading are also presented in Chapter 6 (Equations 6.1b-d and 6.3b-e) and as below. Note that the expressions are functions of both material properties and sandwich column geometric parameters.

- Elastic global buckling load under quasi-static loading (Eqns. 6.1b and 6.3b)

$$
P=\frac{\frac{2 k^{4} \pi^{4} D_{e l}^{(f)} D_{0, e l}}{L^{4}}+\frac{k^{2} \pi^{2} D_{e l}^{(\text {Perp })}}{L^{2}} S_{e l}^{(\text {Perp })}}{\frac{k^{2} \pi^{2} D_{0, e l}}{L^{2}}+S_{e l}^{(\text {Perp })}}
$$

- Elastic face wrinkling load under quasi-static loading (Eqns. 6.1c and 6.3c)

$$
P=\frac{k_{1}^{2} \pi^{2} E}{24 \cos ^{2}(w)\left(1-v^{2}\right)} \frac{h^{3}}{l^{2}}
$$

- Face yield load under quasi-static loading (Eqns. 6.1d)

$$
P=\frac{2 h \sigma_{Y}}{\sqrt{1-v_{P}+v_{P}^{2}}}
$$

- Elastic global buckling load under quasi-static loading (Eqn. 6.3d)

$$
P=\frac{\frac{2 k^{4} \pi^{4} D_{p l}^{(f)} D_{0, p l}}{L^{4}}+\frac{k^{2} \pi^{2} D_{p l}^{(\text {Perp })}}{L^{2}} S_{p l}^{(\text {Perp })}}{\frac{k^{2} \pi^{2} D_{0, p l}}{L^{2}}+S_{p l}^{(\text {Perp })}}
$$

- Plastic global buckling load under quasi-static loading (Eqn. 6.3e) 


$$
P=\frac{k_{1}^{2} \pi^{2} b E_{t}^{\prime}}{24 \cos ^{2}(w)} \frac{h^{3}}{l^{2}}
$$

Here $D_{e l}^{(f)}, S_{e l}^{(p e r p)}, D_{e l}^{(p e r p)}$ are given in Eqns. (3.4a), (2.14), (2.12a), respectively. $\mathrm{D}_{0, \mathrm{el}}$ is the flexural rigidity of the sandwich column faces about the column centroids, given by $D_{0, e l}=\frac{E h(h+l \sin (\omega))^{2}}{2\left(1-v^{2}\right)} ;$ For $D_{p l}^{(f)}, D_{P l}^{(p e r p)}, \mathrm{D}_{0, \mathrm{p}}, \quad \mathrm{E} /\left(1-v^{2}\right)$ should be replaced by planestrain tangent modulus, $E_{T}^{\prime}$ from $D_{e l}^{(f)}, D_{e l}^{(p e r p)}, \mathrm{D}_{0, \mathrm{el}} . S_{e l}^{(\text {perp })}$ and $S_{P l}^{(p e r p)}$ are given in Eqn. (2.14).; $\mathrm{k}$ and $\mathrm{k}_{1}$ are a buckling coefficient for global buckling and a face wrinkling buckling coefficient. Here, $\mathrm{k}=2$ and $\mathrm{k}_{1}=2$ are employed.

\subsection{DYNAMIC EFFECTS ON FAILURE MECHANISM MAPS}

\subsubsection{Movement of Elastic-Plastic Boundaries (Al6061-T6)}

Two boundaries of the Al6061-T6 corrugated core sandwich columns failure map are analyzed with respect to applied compression velocity using the semi-analytical formulations derived earlier..

The movement of the boundary between global elastic buckling and face yield in the failure mechanism map of A16061-T6 corrugated core sandwich columns is shown to depend on compressive velocities (Fig. 5.3). The boundaries for $\mathrm{V}=0.1,0.5$ and $0.7 \mathrm{~m} / \mathrm{s}$ are created by finding geometric points with the global buckling analytical model on a trial-and-error basis. Global curvature imperfections are assigned in the form of the first eigenmode with a magnitude of $\xi=0.05 \mathrm{c}$. The region of face yield tends to expand, highlighting sensitivity to compression velocity. The boundary for $\mathrm{V}=0.1 \mathrm{~m} / \mathrm{s}$ lies even under the quasi-static boundary. That is because the quasi-static boundary is created from 
bifurcation analysis ignoring the effect of imperfections while the effect of global curvature imperfection is included in the analysis of the boundary movement for the case of $\mathrm{V}=0.1 \mathrm{~m} / \mathrm{s}$.

The boundary between elastic face wrinkling (EFW) and face yield (FY) in the failure map of Al6061-T6 corrugated core sandwich columns is analyzed in Fig. 5.4. The face wrinkling analytical model is employed to create the boundaries for compressive velocities $\mathrm{V}=1,2 \mathrm{~m} / \mathrm{s}$ by finding corresponding points on a trial-and-error basis. The first eigenmode with a magnitude of $\xi_{0}^{(f)}=0.01 h$ is assigned to the geometric curvature imperfections of face sheet ligaments.

The region of face yield tends to expand toward the region of EFW as the compressive velocity increases; however, it is insensitive compared with the boundary between EGB and FY. That is, the boundary between EGB and FY moves substantially
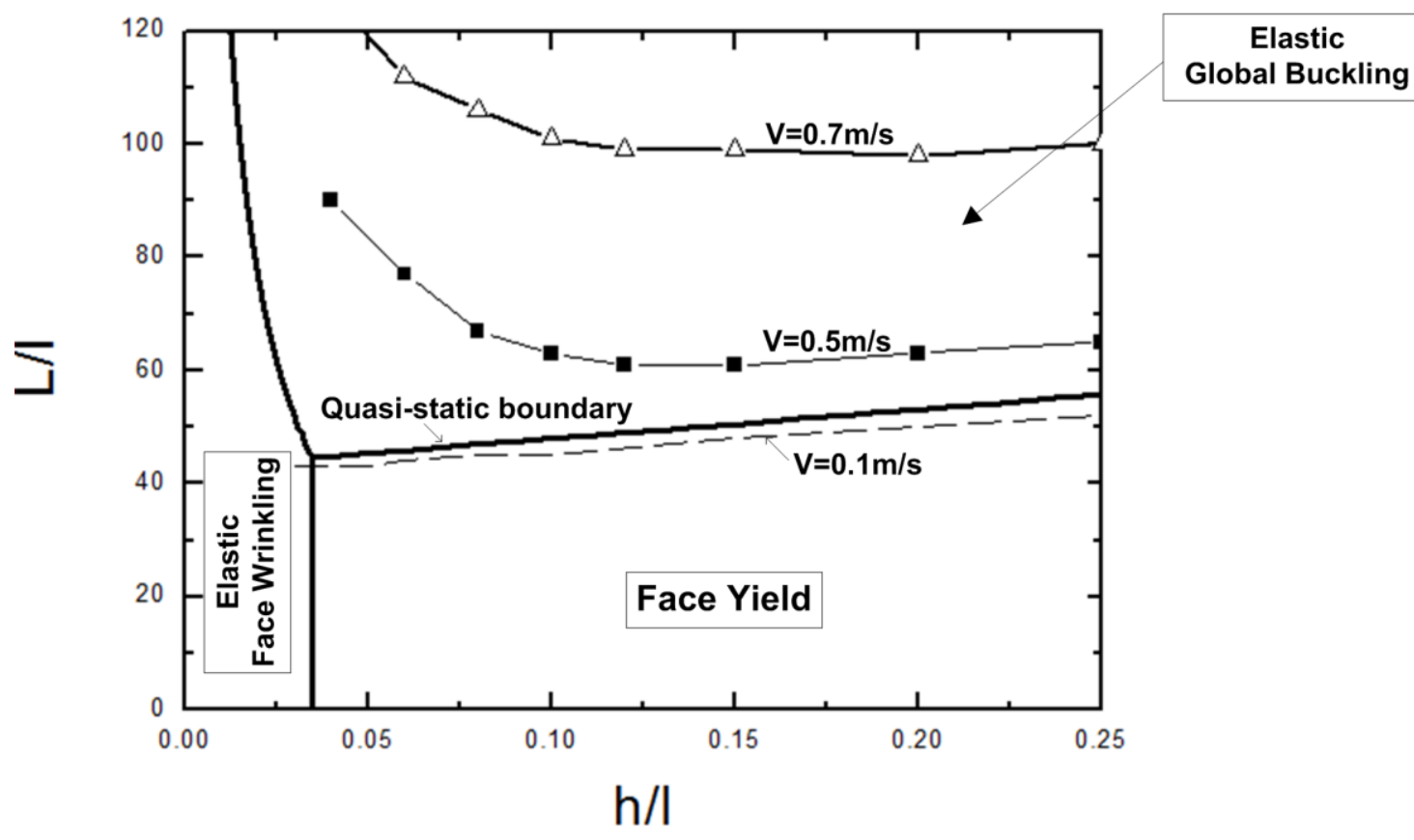

Figure 5.3: Dynamic effects on the boundary between elastic global buckling and face yield of Al6061-T6 corrugated core sandwich columns. 
under compressive velocities less than $\mathrm{V}=1 \mathrm{~m} / \mathrm{s}$. On the other hand, FY would not expand even in $\mathrm{V}=2 \mathrm{~m} / \mathrm{s}$. This observation can be accounted for by a non-dimensional time parameter, $\overline{\mathrm{a}}=\left(\frac{\pi^{4} E I}{\rho A L^{4}}\right)^{1 / 2}$. The parameter determines the rate of the growth of the fundamental mode in an elastic monolithic solid column subject to a constant load P [33] Let $\frac{D_{F W}}{D_{G B}} \sim \frac{h^{3}}{h(l \sin \omega)^{2}}=\left(\frac{h}{l \sin \omega}\right)^{2} ; \frac{\left(\rho A_{G B}\right)}{(\rho h)} \sim \frac{2 h+\bar{\rho} l \sin \omega}{h} ;\left(\frac{L}{(2 l \cos \omega)}\right)^{4}>>1$, and compare the magnitude of the parameters in face wrinkling motion and global buckling motion,

$$
\frac{\bar{a}_{F W}}{\bar{a}_{G B}}=\frac{\left(\frac{\pi^{4} D_{F W}}{\rho h(2 l \cos \omega)^{4}}\right)^{1 / 2}}{\left(\frac{\pi^{4} D_{G B}}{\rho A_{G B} L^{4}}\right)^{1 / 2}}=\sqrt{\left(\frac{D_{F W}}{D_{G B}}\right)\left(\frac{A_{G B}}{h}\right)\left(\frac{L}{(2 l \cos \omega)}\right)^{4}}
$$

The ratio in Eqn (5.6) is much greater than one because of $\left(\frac{L}{2 l \cos \omega}\right)^{4}$, which means

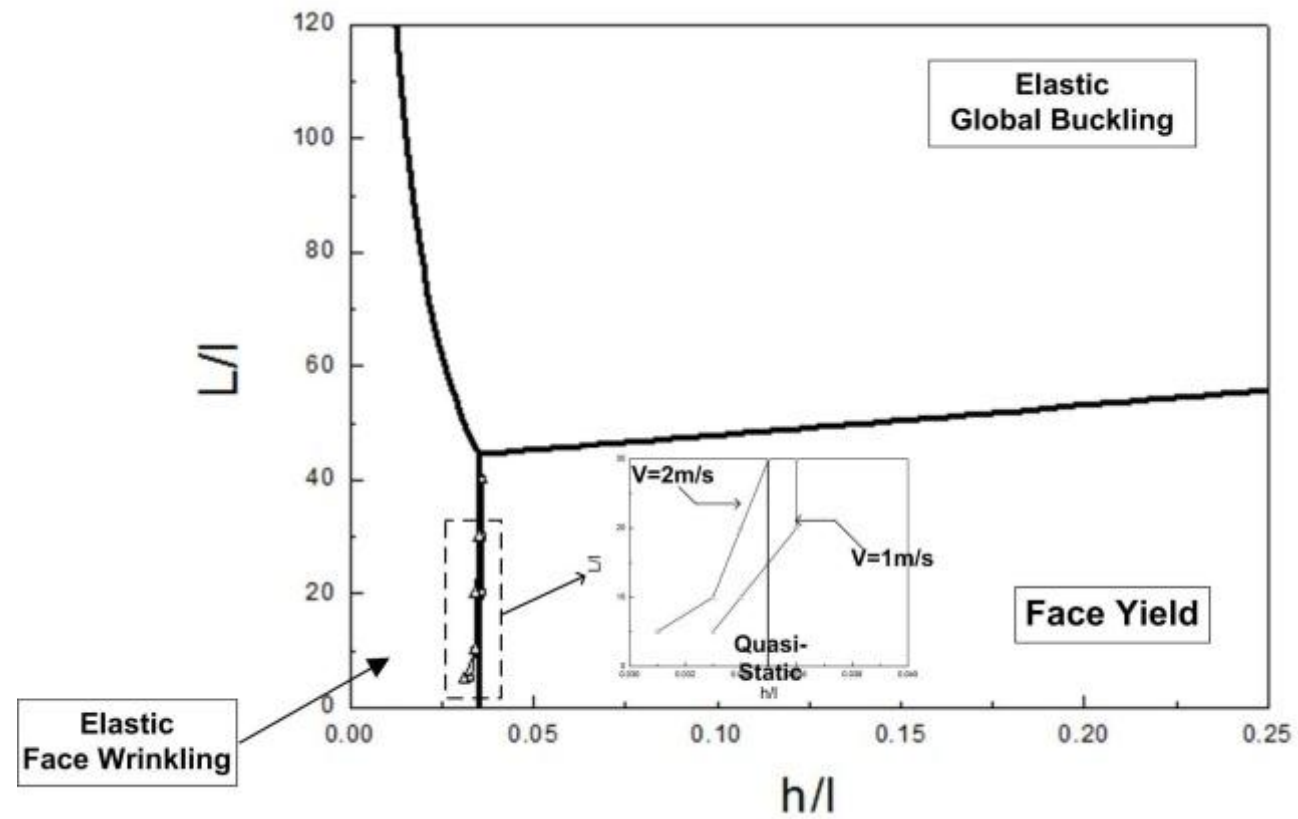

Figure 5.4: Dynamic effects on the boundary between elastic face wrinkling and face yield of Al6061-T6 corrugated core sandwich columns. 
the growth rate of buckling motion in face wrinkling is much greater than in global buckling. From the parameter comparison, it can be deduced that the face wrinkling motion grows rapidly as soon as reaction forces reach a critical value whereas global buckling motion grows more slowly so that it allows a certain grace period until the load drops precipitously. It is for this reason that global buckling failure is more affected by inertial stabilization than face wrinkling failure.

Even though the parameter, $\overline{\mathrm{a}}$, has been introduced from a constant load problem of an elastic monolithic solid column assuming unimodal transverse displacement, it is instructive to compare the rates of growth of two competing failure modes to explain the phenomena shown here.

\subsubsection{Change of Failure Modes Due To Velocity (SS304)}

The change of failure mode between plastic global buckling (PGB) and plastic face wrinkling (PFW) of SS304 corrugated core sandwich columns is studied due to dynamic effects. It is hypothesized that local buckling motion (PFW) predominates global buckling motion as compression velocity increases. Thus, one geometric point in the SS304 failure map is probed to identify failure modes depending on applied velocity rather than creating failure map boundaries as a function of the compression velocity.

Consider Point \#1 shown in Fig. 5.5(a), which represents a sandwich column design whose dominant failure mode is PGB under quasi-static loading. Using the developed analytical models for face wrinkling and global buckling, the time to failure (i.e. loss of load carrying capacity) for each individual failure mode is evaluated for the 
SS304 corrugated core sandwich column of Point \#1 under compression velocities of 1$6 \mathrm{~m} / \mathrm{s}$. Global curvature imperfections in the form of the first eigenmode with a magnitude of $\xi=0.05 \mathrm{c}$ are assigned for the global buckling motion of sandwich columns whereas the asymptotic approach for geometrically perfect faces is used for face wrinkling explained in Chapter 3.

In Fig. 5.5(b), calculation results for the failure time of the SS304 corrugated core sandwich column of Point \#1 obtained from the two analytical models are shown. In velocities less than $3 \mathrm{~m} / \mathrm{s}$, the global buckling prediction to time-to-load drop is earlier than with the face wrinkling prediction. It is assumed that the sandwich column will fail by a failure mode with a predicted time-to-failure that is earlier than the other failure mode. Accordingly, the sandwich column of geometry corresponding to Point \#1 fails by global buckling under a velocity less than $3 \mathrm{~m} / \mathrm{s}$. On the other hand, face wrinkling dominates the sandwich column of Point \#1 at a velocity higher than $3 \mathrm{~m} / \mathrm{s}$.

To confirm the change of failure modes due to the rate-of-compression, finite element analysis of the sandwich column of Point \#1 geometry is carried out for the velocities of $1 \mathrm{~m} / \mathrm{s}$ and $5 \mathrm{~m} / \mathrm{s}$. The FE failure profiles are in agreement with the above analysis as shown in Fig. 5.5(c). The local buckling failure from global buckling failure is expected as compression velocity increases. 


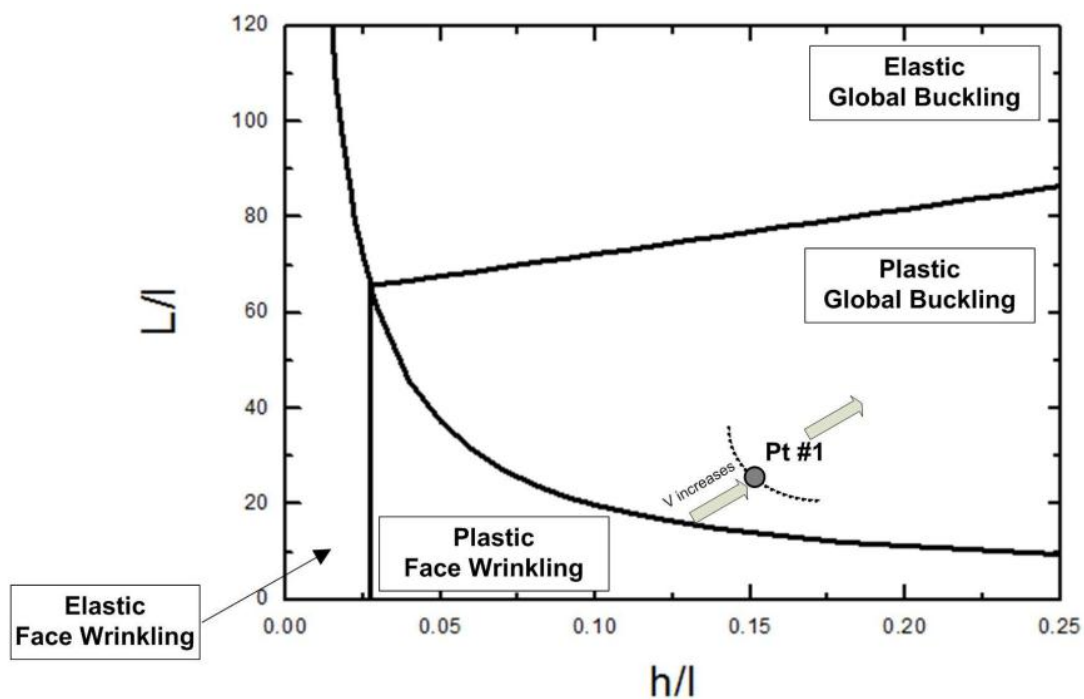

(a)

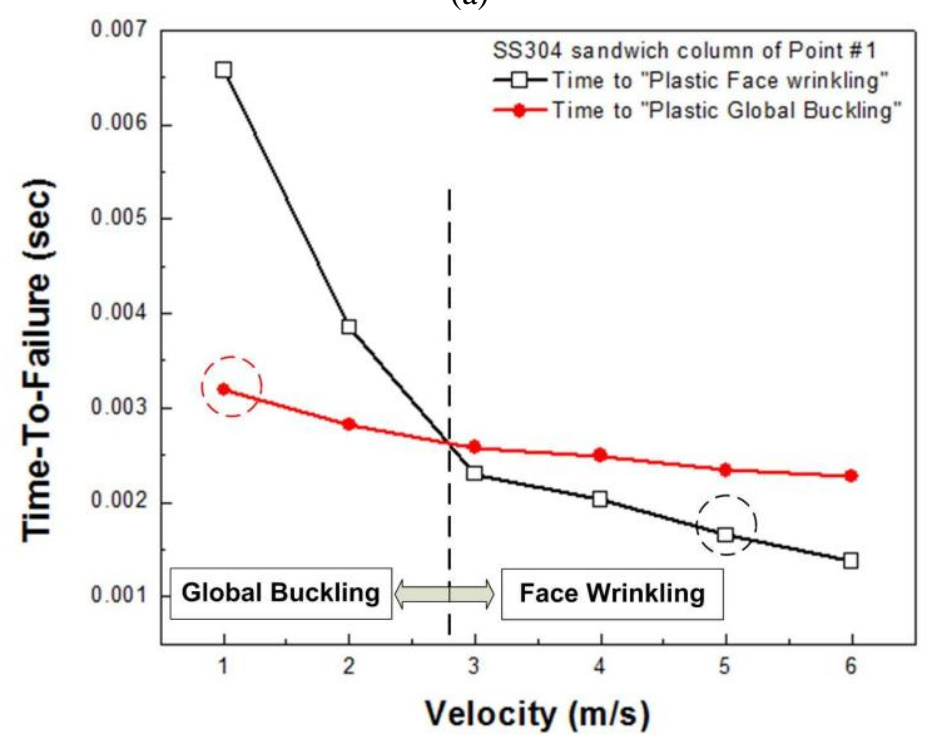

(b)

\section{$V=1 \mathrm{~m} / \mathrm{s}(\mathrm{Pt}$ 1)}

\section{$V=5 \mathrm{~m} / \mathrm{s}$ (Pt 1)}

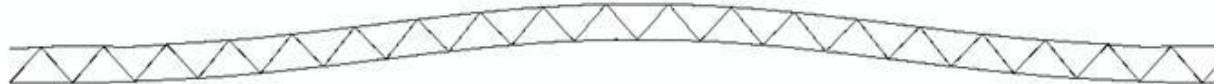

Figure 5.5: Dynamic effects on the change of failure modes from global buckling to local buckling: (a) Quasi-static failure map of SS304 corrugated core sandwich columns with $t=1.5,1=25.0, w=45^{\circ}(\bar{\rho}=12 \%)$ and a geometric point of PT\#1; (b) Calculation of time-to-failure by individual failure modes of face wrinkling and global buckling against applied velocity ranging from $\mathrm{V}=1 \mathrm{~m} / \mathrm{s}$ to $\mathrm{V}=6 \mathrm{~m} / \mathrm{s}$.; (c) FE profiles of the sandwich columns corresponding to PT\#1 under applied velocities $\mathrm{V}=1$ and $5 \mathrm{~m} / \mathrm{s}$. 


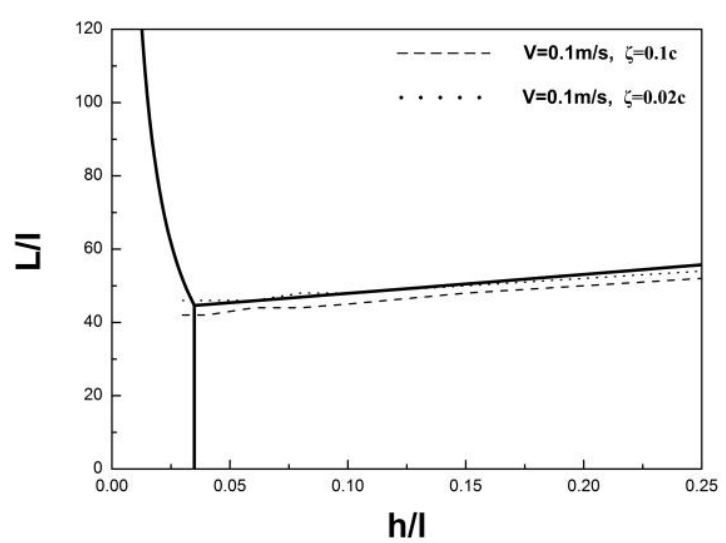

(a)

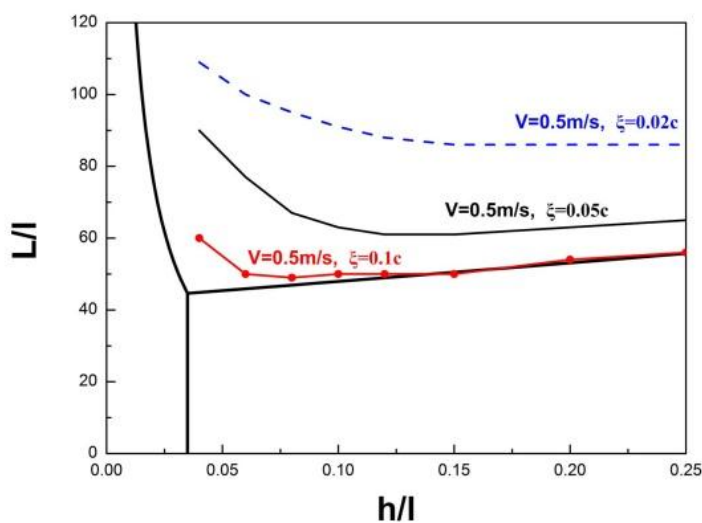

(b)

Figure 5.6: Effect of imperfections on a failure map boundary of A16061-T6 corrugated core sandwich columns under the velocities of (a) $\mathrm{V}=0.1 \mathrm{~m} / \mathrm{s}$, and (b) $\mathrm{V}=0.5 \mathrm{~m} / \mathrm{s}$.

\subsubsection{Effects of Imperfections}

In this subsection, the relationship between imperfections and compression rate on the failure map of Al6061-T6 corrugated core sandwich columns is investigated. The investigation focuses on the boundary between elastic global buckling and face yield which is the most sensitive to compression velocity. The effect of imperfection magnitude for $\mathrm{V}=0.1 \mathrm{~m} / \mathrm{s}$ is shown in Fig. 5.6(a). At this compression velocity $\mathrm{V}=0.1 \mathrm{~m} / \mathrm{s}$, face yield region occupies a slightly larger region with smaller magnitude of imperfections $(\xi=0.02 \mathrm{c})$ than with larger magnitude of imperfections $(\xi=0.1 \mathrm{c})$. However, the difference is not significant.

On the other hand, the boundary for a compression velocity of $\mathrm{V}=0.5 \mathrm{~m} / \mathrm{s}$ is much more sensitive to imperfection magnitudes as shown in Fig. 5.7(b). This observation is quite similar to the Type II response of Calladine's studies [62, 88]. See Fig 5.7 for the definition of Type I and Type II structures. A compression structure whose dominant deformation is axial deformation (Type II structure) rather than bending deformation (Type I structure), is known to be sensitive to imperfections and velocity. 

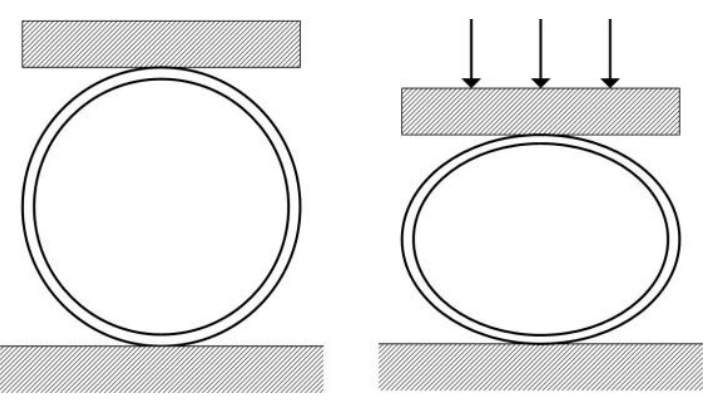

(a)
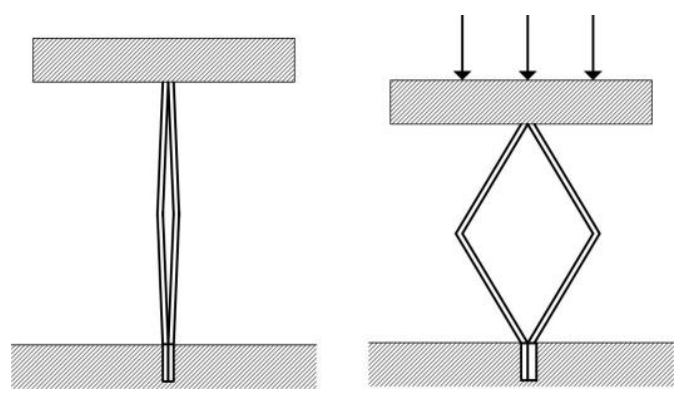

(b)

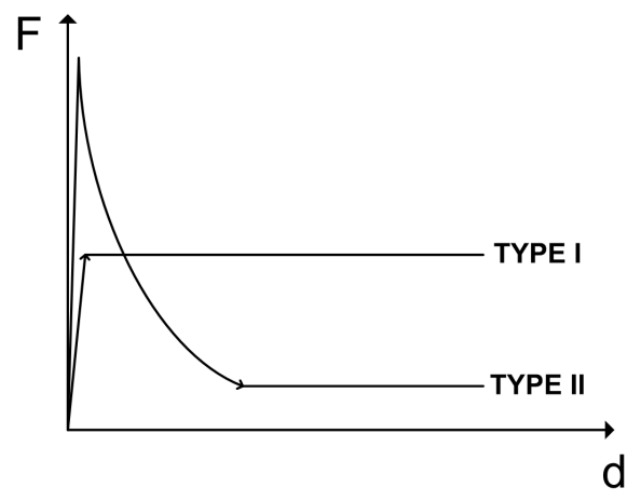

(c)

Figure 5.7: (a) Type I; (b) Type II; (c) Quasi-static load-deflection curve of Type I and Type II structures according to Calladine and English's notation[62,88].

\subsection{CHAPTER SUMMARY}

Dynamic effects on the two quasi-static failure maps of corrugated core sandwich columns compressed perpendicular-to-corrugations are analyzed as rate-of-compression increases. To obtain the dynamic response of individual failure modes, analytical models developed in Part I of this dissertation are employed. The analysis in this chapter is summarized below.

(1) For increasing loading rates, columns designed to fail by global buckling are more robust than those designed to fail by face wrinkling due to inertia stabilization: Global buckling motion is more resistible compared with face wrinkling as rate-ofcompression increases due to inertial stabilization of global buckling motion. The 
boundary between EGB and FY in the Al6061-T6 corrugated core sandwich columns is shown to be sensitive to compressive velocity.

(2) With increased rate-of-compression, face wrinkling failure mode becomes dominant.

(3) The effects of imperfections of sandwich columns are more significant with increased rate-of-loading as has been found in simple compressive structures $[62,88]$. 


\section{CHAPTER 6}

\section{DYNAMIC OPTIMIZATION}

\subsection{INTRODUCTION}

The dynamic effects on minimum weight designs of corrugated core sandwich columns are considered. The majority of previous optimal design studies for periodic core sandwich structures concern quasi-static loading under which dynamic effects are neglected [28, 89-91]. However, it is obvious that the optimal designs based on quasistatic analysis would not work well under an increased rate-of-loading, up to a few $\mathrm{m} / \mathrm{s}$ in the low velocity region as specified previously.

Two specific objectives are established in this chapter. The first one is to understand the influence of dynamic loading on the competing failure mechanisms. The second is to provide a methodology for optimal design of corrugated core sandwich columns in both in-plane loading directions considering dynamic effects under the circumstances that there is no explicit expression for dynamic response, and to investigate how the optimal design variables evolve as the loading rate increases.

The organization of this chapter is as follows:

In Section 6.2, quasi-static optimization problems regarding corrugated core sandwich columns made of Al6061-T6 and SS304 will be established and solved for two in-plane loading orientations: perpendicular-to-corrugations and parallel-tocorrugations. The solutions to the quasi-static optimization problems will be taken as references for the following two sections. 
In Section 6.3, how the quasi-static optimal designs and their core-height-varied designs of equivalent mass respond to dynamic loads will be investigated employing FEM and the analytical approaches developed in the previous chapters 2 and 3 . Thus, the competing failure mechanisms under dynamic circumstances will be understood.

In Section 6.4, an optimization procedure for minimum weight designs of Al6061T6 corrugated core sandwich columns under dynamic in-plane loading will be conducted using the analytical approaches, response surface methodology [92], and a sequential quadratic programming (SQP) optimization algorithm [93,94]. The fundamental difference between optimization based on quasi-static and that based on dynamic loading is that dynamic response cannot be expressed by an explicit function of design variables. This is due to the complex phenomena that occur as the loading rate increases. It is therefore necessary to approximate the dynamic response. To do this, response surface methodology (RSM) [92] is introduced in order to approximate the dynamic response in terms of design variables, and an optimization problem based on the approximated dynamic responses of sandwich columns is solved using the SQP algorithm supported by commercial optimization software, Altair/Hyperstudy v10.0 [95].

Throughout this chapter, the length of columns will be prescribed as $\mathrm{L}=1 \mathrm{~m}$ rather than introducing dimensionless parameters.

\subsection{QUASI-STATIC OPTIMAL DESIGNS}

The quasi-static optimization study will consider loading the corrugated core sandwich column in both the perpendicular-to-corrugations and parallel-to-corrugations. Two materials will be considered-Al6061-T6 and SS304. The main differences 
between this study and those carried out by Biagi et al [28] are three points; (a) performing a standard optimization procedure, (b) considering more design variables (i.e., corrugation angle, w, is included as a design variable), (c) using a simpler description of plastic region (by linear strain-hardening).

The quasi-static optimal design problem is to solve for the minimum mass of a structure and material choice under a given load, while the possible active failure modes are suppressed. Accordingly, the optimization problems for the four types are established in Equations (6.1)-(6.4). In all sets of equations, an objective function, f, represents total mass, and a design constraint, $\mathrm{g}_{\mathrm{i}}$, stands for a load which should be sustained by the corresponding i-th failure mechanism. Material systems and in-plane loading orientations are written in the superscripts of $\mathrm{f}$ and $\mathrm{g}$.

The optimal design problem formulations for the four types of problems are given in the sets of equations (6.1)-(6.4) which are based on Biagi's quasi-static analysis on corrugated core sandwich columns [28].

Al6061-T6 is treated as an elastic-perfectly plastic material unlike the material modeling in Chapters 2,3, and 4. The material modeling stems from the fact that an Al6061-T6 corrugated core sandwich column does not allow much post-yield deformation, especially in quasi-static compression [27,28]. As a result, there was no significant difference when Al6061-T6 was considered as an elastic-perfectly plastic material or as an elastic-strain hardening material according to Biagi's study [28].

Regarding the material modeling of SS304, the quasi-static optimal design problem in this study considers one strain hardening modulus $\left(E_{P}^{(1)}=\frac{d \sigma_{e q}}{d p}=8117.65(M P a)\right)$ for a small plastic strain region $(0<\mathrm{p}<0.0164)$. Therefore, the plastic response of SS304 can 
be expressed as shown in Eqns. (6.3d), (6.3e), (6.4e), (6.4f), (6.4g). More detail regarding the quasi-static analysis can be found in Biagi's study [27, 28, 103] The four types of optimal design problems given in Eqn. (6.1) through Eqn. (6.4) are solved using the optimization algorithm, Sequential Quadratic Programming (SQP), supported by a commercial optimization software program, Altair/Hyperstudy v10. SQP, a gradientbased optimization algorithm, has excellent convergence performance and an advantage in that an initial design point even positioned in an infeasible design domain eventually converges to an optimal solution $[93,94]$. Not only design variables, $\mathrm{h}, \ell, \mathrm{t}$ but also corrugation angle, $\mathrm{w}$, is included and found in the optimal design problems unlike Biagi's optimization [28]. 
- Optimization problem of Al6061-T6 corrugated core sandwich columns quasistatically loaded perpendicular-to-corrugations.

$$
\text { To minimize } \quad f^{(A l-p e r p)}(h, l, t, w)=2 b L \rho\left(h+\frac{t}{2 \cos (w)+\frac{2 t}{l \sin (w)}}\right)
$$

Subject to

Elastic global buckling [28] :

$$
g_{1}^{(A l-p e e p)}(h, l, t, w)=\frac{\frac{2 k^{4} \pi^{4} D_{e l}^{(f)} D_{0, e l}}{L^{4}}+\frac{k^{2} \pi^{2} D_{e l}^{(P e r p)}}{L^{2}} S_{e l}^{(P e r p)}}{\frac{k^{2} \pi^{2} D_{0, e l}}{L^{2}}+S_{e l}^{(P e r p)}}>P
$$

Elastic face wrinkling [28]: $\quad g_{2}^{(A l-p e r p)}(h, l, t, w)=\frac{k_{1}^{2} \pi^{2} E}{24 \cos ^{2}(w)\left(1-v^{2}\right)} \frac{h^{3}}{l^{2}}>P$

Yield [28] : $\quad g_{3}^{(A l-p e r p)}(h, l, t, w)=\frac{2 h \sigma_{Y}}{\sqrt{1-v_{P}+v_{P}^{2}}}>P$

where $D_{e l}^{(f)}, S_{e l}^{(p e r p)}, D_{e l}^{(p e r p)}$ are given in Eqns. (3.4a), (2.14), (2.12a), respectively. $\mathrm{D}_{0, \mathrm{el}}$ is the flexural rigidity of the sandwich column faces about the column centroids, given by $D_{0, e l}=\frac{E h(h+l \sin (\omega))^{2}}{2\left(1-v^{2}\right)} \cdot \mathrm{k}$ and $\mathrm{k}_{1}$ are a buckling coefficient for global buckling and a face wrinkling buckling coefficient. Here, $\mathrm{k}=2$ and $\mathrm{k}_{1}=2$ are employed. 
- Optimization problem of Al6061-T6 corrugated core sandwich columns quasistatically loaded parallel-to-corrugations.

$$
\text { To minimize } \quad f^{(A l-p a r a)}(h, l, t, w)=2 b L \rho\left(h+\frac{t}{2 \cos (w)+\frac{2 t}{l \sin (w)}}\right)
$$

Subject to

Elastic global buckling [28]:

$$
g_{1}^{(A l-p a r a)}(h, l, t, w)=\frac{\frac{2 k^{4} \pi^{4} D_{e l}^{(f)} D_{0, e l}}{L^{4}}+\frac{k^{2} \pi^{2} D_{e l}^{(\text {Para })}}{L^{2}} S_{e l}^{(\text {Para })}}{\frac{k^{2} \pi^{2} D_{0, e l}}{L^{2}}+S_{e l}^{(\text {Para })}}>P
$$

Elastic local face plate buckling [28]:

$$
g_{2}^{(A l-\text { para })}(h, l, t, w)=\frac{K^{2} \pi^{2} E h^{2} A^{(\text {extruded })}}{48\left(1-v^{2}\right) l^{2} \cos ^{2}(w)}>P
$$

Elastic local core plate buckling [28] :

$$
g_{3}^{(A l-p a r a)}(h, l, t, w)=\frac{K^{2} \pi^{2} E t^{2} A^{(e x t r u d e d)}}{12\left(1-v^{2}\right) l^{2}}>P
$$

Yield [28]:

$$
g_{4}^{(A l-p a r a)}(h, l, t, w)=\frac{\sigma_{Y}}{\sqrt{1-v_{P}+v_{P}^{2}}}\left(\frac{t}{\cos (w)+\frac{t}{l \sin (w)}}+2 h\right)>P
$$

where $D_{e l}^{(f)}, S_{e l}^{(\text {para })}, D_{e l}^{(\text {para })}$ are given in Eqns. (3.4a), (2.18), (2.15), respectively. $\mathrm{D}_{0, \mathrm{el}}$ is the flexural rigidity of the sandwich column faces about the column centroids, given by $D_{0, e l}=\frac{E h(h+l \sin (\omega))^{2}}{2\left(1-v^{2}\right)} \cdot \mathrm{A}^{\text {(extruded) }}$ is the cross-sectional material area for A16061-T6 corrugated core sandwich columns manufactured by extrusion given by $A^{(\text {extruded })}=\frac{t}{\cos (\omega)+t /(l \sin (\omega))}+2 h \quad$ [28]. And, $\mathrm{k}$ and $\mathrm{K}$ are a buckling coefficient for global buckling and a local plate buckling coefficient, respectively. Here, $\mathrm{k}=2$, $\mathrm{K}=7$ are employed. 
- Optimization problem of SS304 corrugated core sandwich columns quasi-statically loaded perpendicular-to-corrugations.

$$
\text { To minimize } f^{(S S-p e r p)}(h, l, t, w)=2 b L \rho\left(h+\frac{t}{2 \cos (w)}\right)
$$

Subject to

Elastic global buckling [28] :

$$
g_{1}^{(S S-p e r p)}(h, l, t, w)=\frac{\frac{2 k^{4} \pi^{4} D_{e l}^{(f)} D_{0, e l}}{L^{4}}+\frac{k^{2} \pi^{2} D_{e l}^{(\text {Perp })}}{L^{2}} S_{e l}^{(\text {Perp })}}{\frac{k^{2} \pi^{2} D_{0, e l}}{L^{2}}+S_{e l}^{(\text {Perp })}}>P
$$

Elastic face wrinkling [28]: $\quad g_{2}^{(S S-p e r p)}(h, l, t, w)=\frac{k_{1}^{2} \pi^{2} E}{24 \cos ^{2}(w)\left(1-v^{2}\right)} \frac{h^{3}}{l^{2}}>P$

Plastic global buckling [28]:

$$
g_{3}^{(S S-p e r p)}(h, l, t, w)=\max \left(\frac{\frac{2 k^{4} \pi^{4} D_{p l}^{(f)} D_{0, p l}}{L^{4}}+\frac{k^{2} \pi^{2} D_{p l}^{(\text {Perp })}}{L^{2}} S_{p l}^{(\text {Perp })}}{\frac{k^{2} \pi^{2} D_{0, p l}}{L^{2}}+S_{p l}^{(\text {Perp })}}, \frac{2 h \sigma_{Y}}{\sqrt{1-v_{P}+v_{P}^{2}}}\right)>P
$$

Plastic face wrinkling [28]: $g_{4}^{(S S-p e r p)}(h, l, t, w)=\max \left(\frac{k_{1}^{2} \pi^{2} b E_{t}^{\prime}}{24 \cos ^{2}(w)} \frac{h^{3}}{l^{2}}, \frac{2 h \sigma_{Y}}{\sqrt{1-v_{P}+v_{P}^{2}}}\right)>P$

Here $D_{e l}^{(f)}, S_{e l}^{(\text {perp })}, D_{e l}^{(\text {perp })}$ are given in Eqns. (3.4a), (2.14), (2.12a), respectively. $\mathrm{D}_{0, \mathrm{el}}$ is the flexural rigidity of the sandwich column faces about the column centroids, given by $D_{0, e l}=\frac{E h(h+l \sin (\omega))^{2}}{2\left(1-v^{2}\right)}$; For $D_{p l}^{(f)}, D_{P l}^{(p e r p)}, \mathrm{D}_{0, \mathrm{pl}}$ in $(6.3 \mathrm{~d}), \quad \mathrm{E} /\left(1-v^{2}\right)$ should be replaced by plane-strain tangent modulus, $E_{T}^{\prime}$ from $D_{e l}^{(f)}, D_{e l}^{(\text {perp })}, \mathrm{D}_{0, \mathrm{el}} \cdot S_{e l}^{(\text {perp })}$ and $S_{P l}^{(\text {perp })}$ are given in Eqn. (2.14). 
- Optimization problem of SS304 corrugated core sandwich columns quasi-statically loaded parallel-to-corrugations

$$
\text { To minimize } f^{(S S-p a r a)}(h, l, t, w)(h, l, t, w)=2 b L \rho\left(h+\frac{t}{2 \cos (w)}\right)
$$

Elastic global buckling [28]:

$$
g_{1}^{(S S-p e r p)}(h, l, t, w)=\frac{\frac{2 k^{4} \pi^{4} D_{e l}^{(f)} D_{0, e l}}{L^{4}}+\frac{k^{2} \pi^{2} D_{e l}^{(\text {Para })}}{L^{2}} S_{e l}^{(\text {Para })}}{\frac{k^{2} \pi^{2} D_{0, e l}}{L^{2}}+S_{e l}^{(\text {Para })}}>P
$$

Elastic local face plate buckling [28] :

$$
g_{2}^{(S S-\text { para })}(h, l, t, w)=\frac{K^{2} \pi^{2} E h^{2} A^{(b e n t)}}{48\left(1-v^{2}\right) l^{2} \cos ^{2}(w)}>P
$$

Elastic local core plate buckling [28] :

$$
g_{3}^{(S S-\text { para })}(h, l, t, w)=\frac{K^{2} \pi^{2} E t^{2} A^{(\text {bent })}}{12\left(1-v^{2}\right) l^{2}}>P
$$

Plastic global buckling [28]:

$$
g_{4}^{(S S-p a r a)}(h, l, t, w)=\max \left(\frac{\frac{2 k^{4} \pi^{4} D_{p l}^{(f)} D_{0, p l}}{L^{4}}+\frac{k^{2} \pi^{2} D_{p l}^{(\text {Para })}}{L^{2}} S_{p l}^{(\text {Para })}}{\frac{k^{2} \pi^{2} D_{0, p l}}{L^{2}}+S_{p l}^{(\text {Para })}}, \frac{A^{(\text {bent })} \sigma_{Y}}{\sqrt{1-v_{P}+v_{P}^{2}}}\right)>P
$$

Plastic local face plate buckling [28]:

$$
g_{5}^{(S S-\text { para })}(h, l, t, w)=\max \left(\frac{K^{2} \pi^{2} E_{P} h^{2} A^{(\text {bent })}}{48\left(1-v_{P}^{2}\right) l^{2} \cos ^{2}(w)}, \frac{A^{(\text {bent })} \sigma_{Y}}{\sqrt{1-v_{P}+v_{P}^{2}}}\right)>P
$$

Plastic local core plate buckling [28]:

$$
g_{6}^{(S S-\text { para })}(h, l, t, w)=\max \left(\frac{k_{1}^{2} \pi^{2} E_{P}}{24\left(1-v_{P}^{2}\right) \cos ^{2}(w)} \frac{h^{3}}{l^{2}}, \frac{A^{(\text {bent })} \sigma_{Y}}{\sqrt{1-v_{P}+v_{P}^{2}}}\right)>P
$$


where $D_{e l}^{(f)}, S_{e l}^{(\text {para })}, D_{e l}^{(\text {para })}$ are given in Eqns. (3.4a), (2.18), (2.15), respectively. $\mathrm{D}_{0, \mathrm{el}}$ is the flexural rigidity of the sandwich column faces about the column centroids, given by $D_{0, e l}=\frac{E h(h+l \sin (\omega))^{2}}{2\left(1-v^{2}\right)} . ;$ For $D_{p l}^{(f)}, D_{P l}^{(p e r p)}, \mathrm{D}_{0, \mathrm{pl}}$ in $(6.4 \mathrm{~d}), \mathrm{E} /\left(1-v^{2}\right)$ should be replaced by plane strain tangent modulus, $E_{t}^{\prime}$ from $D_{e l}^{(f)}, D_{e l}^{(\text {perp })}, \mathrm{D}_{0, \mathrm{el}} . \quad S_{p l}^{(\text {para })}$ is given in Eqn. (2.18) according to Becque's derivation[12]. $\mathrm{D}_{0, \mathrm{pl}}$ is obtained from $\mathrm{D}_{0, \mathrm{el}}$ by replacing $\frac{E}{\left(1-v^{2}\right)}$ by $E_{T}^{\prime} . A^{(\text {bent })}$ is the cross-sectional material area for SS304 corrugated core sandwich columns manufactured by bending/brazaing given by $A^{(\text {bent })}=\frac{t}{\cos (\omega)}+2 h$ [28].

Fig. 6.1 shows the comparison of minimum weight designs of Al6061-T6 and SS304 corrugated core sandwich columns of $\mathrm{L}=1 \mathrm{~m}$ depending on a given load. The results agree with Biagi's optimization results in the following aspects. Perpendicular-tocorrugations is more beneficial than parallel-to-corrugations at a low magnitude of load, and vice versa at a high magnitude of load.

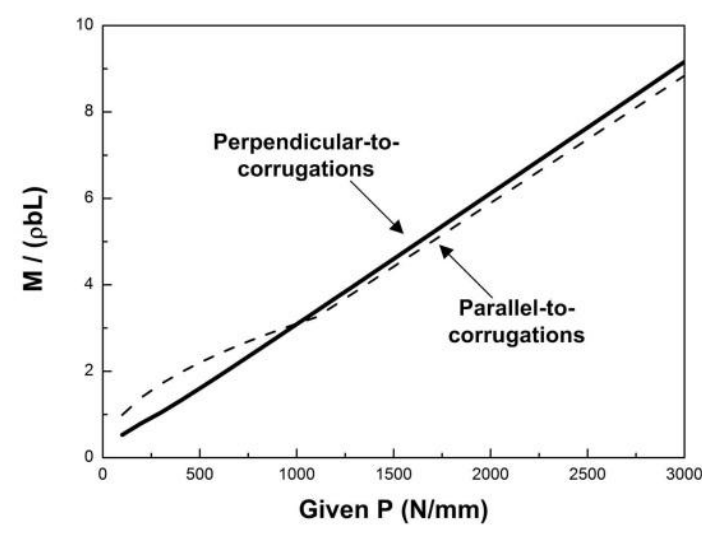

(a)

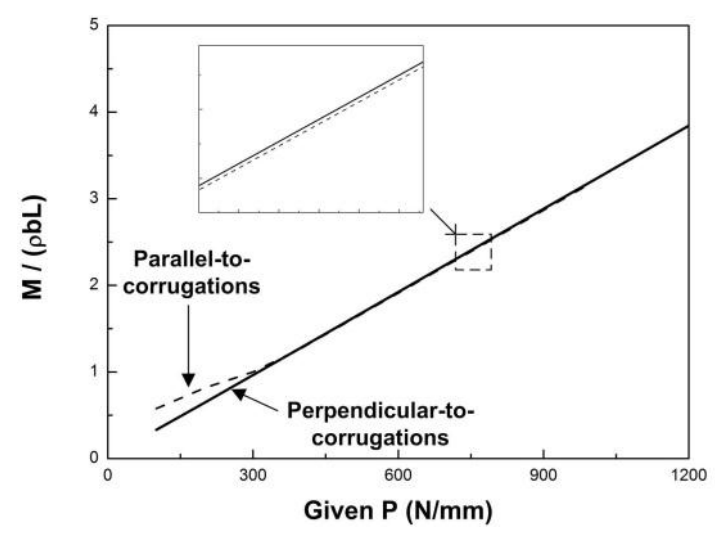

(b)

Figure 6.1: Weight comparison of corrugated core sandwich columns of $\mathrm{L}=1 \mathrm{~m}$ under quasi-static loading: (a) Al6061-T6 corrugated core sandwich columns, and (b) SS304 corrugated core sandwich columns. 
The agreement can be explained as follows. In the low load region where perpendicular-to-corrugation is superior, the yield constraint, $\mathrm{g}_{3}^{(A l-p e r p)}$, as well as the constraints associated with elastic behavior, $\mathrm{g}_{1}^{(A l-p e r p)}$ and $\mathrm{g}_{2}^{(A l-p e r p)}$, are simultaneously active in the perpendicular-to-corrugations orientation while only elastic constraints, $\mathrm{g}_{1}^{(A l-\text { para })}$ through $\mathrm{g}_{3}^{(A l-\text { para })}$ are active in the low magnitude load range in the optimization problem for the parallel-to-corrugations. Generally, the best minimum weight design can be obtained from the simultaneous occurrence of all possible failure modes together with yielding according to naïve optimization [89]. Likewise, this explanation applies to SS304 columns as shown in Fig. 6.1b.

In the higher load region where the parallel-to-corrugation is superior, the superiority can be accounted for by the difference of effective cross-sectional area capable of carrying an in-plane load between the two loading orientations. In the highload region where parallel-to-corrugations is superior, the columns of both of the inplane loading orientations are at the onset of yielding. The weight difference between optimal columns of two loading orientations can be interpreted as the sandwich core cross-section not involved in carrying an in-plane load in the in-plane loading orientation of perpendicular-to-corrugations.

The optimal design variables of the four types of corrugated core sandwich columns of $\mathrm{L}=1 \mathrm{~m}$ depending on a given in-plane load are shown in Fig. 6.2. It is noteworthy that optimal corrugation thickness, t, of optimal sandwich columns compressed perpendicular-to-corrugations is much smaller $(<0.1 \mathrm{~mm}$ for $\mathrm{L}=1 \mathrm{~m})$ than the others as shown in Fig. 6.2 (a) and (c). The extremely small thickness dimension, t, can be also confirmed in Biagi's study if substituting a physical value, $\mathrm{L}=1 \mathrm{~m}$, into the non- 


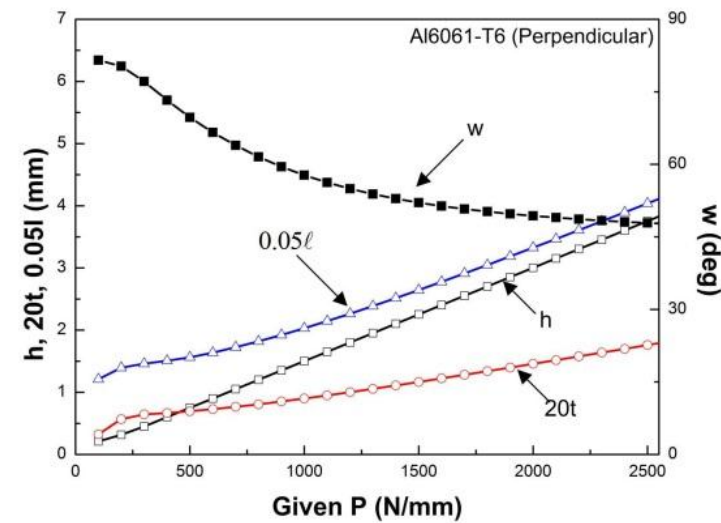

(a)

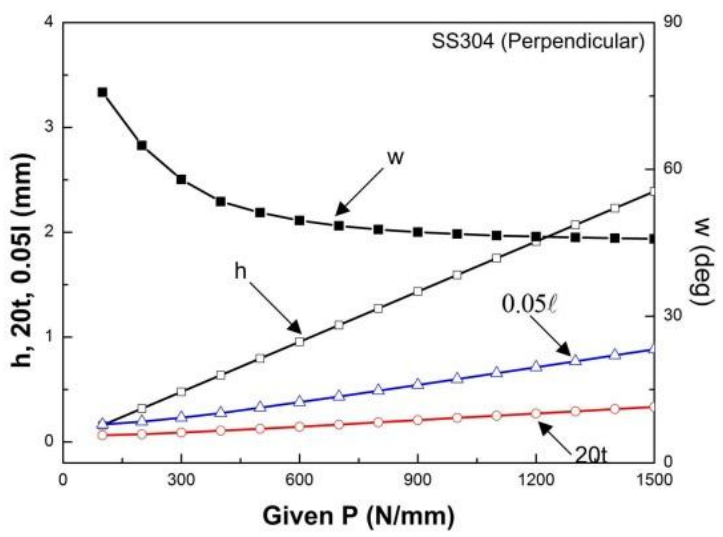

(c)

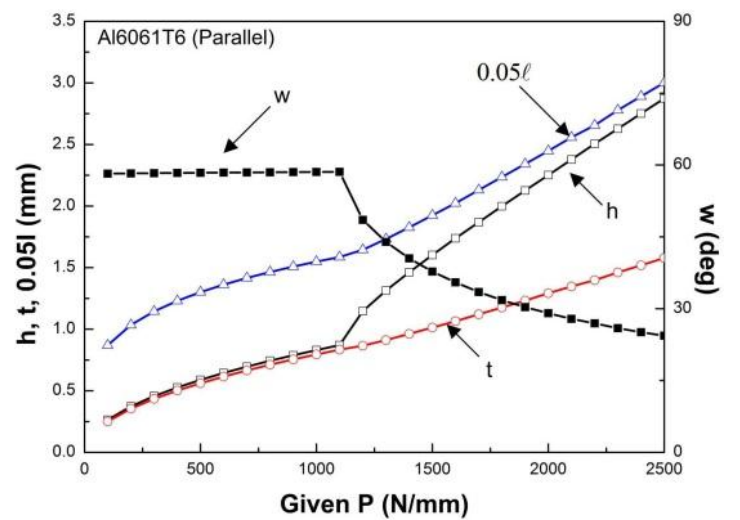

(b)

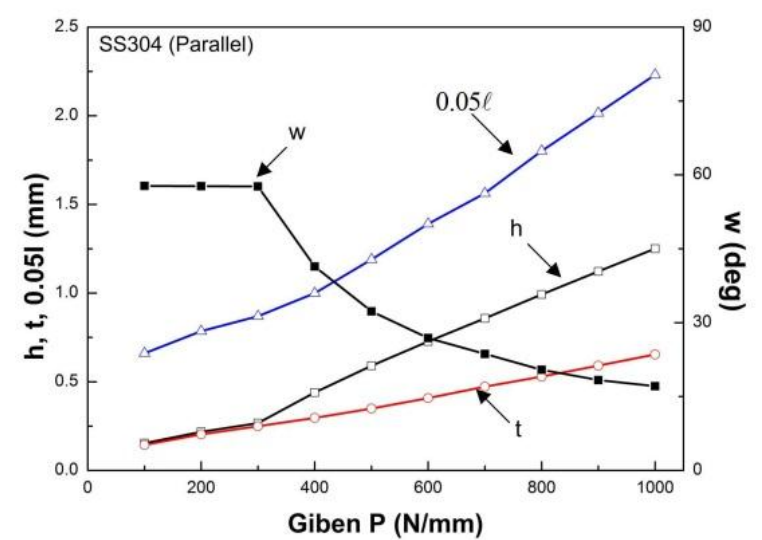

(d)

Figure 6.2: The optimal design variables for quasi-static in-plane loading: (a) Al6061-T6 (perpendicular-tocorrugations); (b) Al6061-T6 (parallel-to-corrugations); (c) SS304 (perpendicular-to-corrugations); (d) SS304 (parallel-to-corrugations).

dimensional parameters in his study [28]. Thus, it may not be prudent to conclude that corrugated core sandwich columns compressed perpendicular-to-corrugations are better than those compressed parallel-to-corrugations for sustaining a low magnitude of load.

Note that the quasi-static optimization results in this section will be reference data for the following sections. 


\subsection{DYNAMIC RESPONSE OF QUASI-STATIC OPTIMAL SOLUTIONS AND}

\section{THEIR MASS-EQUIVALENT DESIGNS}

The quasi-static optimal solutions and their mass-equivalent designs are subjected to dynamic in-plane loading. Let one of the quasi-static optimal designs from each category be denoted by Design I, II, III, and IV. Design I and Design II are minimum weight designs of Al6061-T6 corrugated core sandwich columns of L=1m carrying an inplane compressive load of $\mathrm{P}=1000 \mathrm{~N} / \mathrm{mm}$ in the direction of perpendicular-tocorrugations and parallel-to-corrugations, respectively. Likewise, Design III and Design IV correspond to the optimal SS304 corrugated core sandwich columns of $\mathrm{L}=1 \mathrm{~m}$ subjected to a quasi-static in-plane load of $\mathrm{P}=500 \mathrm{~N} / \mathrm{mm}$. The quasi-static designs (Design $I, I I, I I I$, and $I V)$ are ones of the quasi-static optimal solutions to be tested under dynamic loading. They, though somewhat arbitrarily selected, fail by the simultaneous occurrence of global buckling and local buckling under the quasi-static loads.

First, consider Al6061-T6 corrugated core sandwich columns compressed

Table 6.1. Core-height-varied designs of Design I

\begin{tabular}{cccccc}
\hline Design No. & $\mathrm{h}$ & $\mathrm{t}$ & $\mathrm{l}$ & $\mathrm{w}$ & $\mathrm{M} /(\mathbf{\rho b L})$ \\
\hline Case_Aperp_Base (Design I) & $\mathbf{1 . 5 0 2}$ & $\mathbf{0 . 0 4 5}$ & $\mathbf{4 0 . 6 3}$ & $\mathbf{5 7 . 7 7}$ & $\mathbf{3 . 0 9}$ \\
Case_APerp11 & 1.5 & 0.05513245 & 50 & 60 & 3.11 \\
Case_APerp12 & 1.5 & 0.055148054 & 45 & 60 & 3.11 \\
Case_APerp13* & 1.5 & 0.055167571 & 40 & 60 & 3.11 \\
Case_APerp14 & 1.5 & 0.055192684 & 35 & 60 & 3.11 \\
Case_APerp15 & 1.5 & 0.05522620 & 30 & 60 & 3.11 \\
Case_APerp16 & 1.5 & 0.055273201 & 25 & 60 & 3.11 \\
Case_APerp17 & 1.5 & 0.055343847 & 20 & 60 & 3.11 \\
Case_APerp18 & 1.5 & 0.055461991 & 15 & 60 & 3.11 \\
\hline
\end{tabular}

* Case_Aperp13 is the closest to Design I 
perpendicular-to-corrugations. Using Design I as the base design, eight samples with the core height varied yet retaining the same face sheet thickness, corrugation angle and weight and are listed in Table 6.1. The core-height varied design whose $\ell$ is greater than that of Design I has a greater global static buckling strength but smaller local static buckling strength than Design I. On the contrary, the core height varied design whose $\ell$ is smaller than that of Design I has a smaller global static buckling strength but greater local static buckling strength.

Using the analytical approaches for face wrinkling and global buckling, impulses of the eight listed sandwich column designs are calculated for a compression velocity of $\mathrm{V}=1 \mathrm{~m} / \mathrm{s}$. In all cases, global curvature imperfections and face ligament curvature imperfections are assigned in the form of the first eigenmodes, $\mathrm{w}_{0}(x)=\frac{\xi^{(g)}}{2}(1-$ $\left.\cos \left(\frac{2 \pi x}{L}\right)\right)$ and $w_{0}^{(f)}=\frac{\xi^{(f)}}{2}\left(1-\cos \left(\frac{2 \pi x}{2 \ell \cos (\omega)}\right)\right)$ with magnitudes of $\xi^{(g)}=0.002 L$, $\xi^{(f)}=0.01 h$, respectively. The impulse is obtained from integrating the front end reaction force with respect to time until time to load-drop. For each design, two impulse calculations are carried out employing the face wrinkling and the global buckling analytical models. However, the smaller one between the two calculated impulses is physically meaningful because each design is expected to fail by the failure mode whose calculated impulse is smaller (i.e., either face wrinkling or global buckling).

Fig. 6.3 shows the impulse calculations of the eight mass-equivalent core-height varied designs of $\mathrm{L}=1 \mathrm{~m}$ at $\mathrm{V}=1 \mathrm{~m} / \mathrm{s}$. The blue dash line in the figure represents the maximum impulse capacity under $\mathrm{V}=1 \mathrm{~m} / \mathrm{s}$ sustained by each sandwich design having core height $\mathrm{c}=\ell \cdot \sin \omega$. The design number, Case_APerp13, the design closest to 


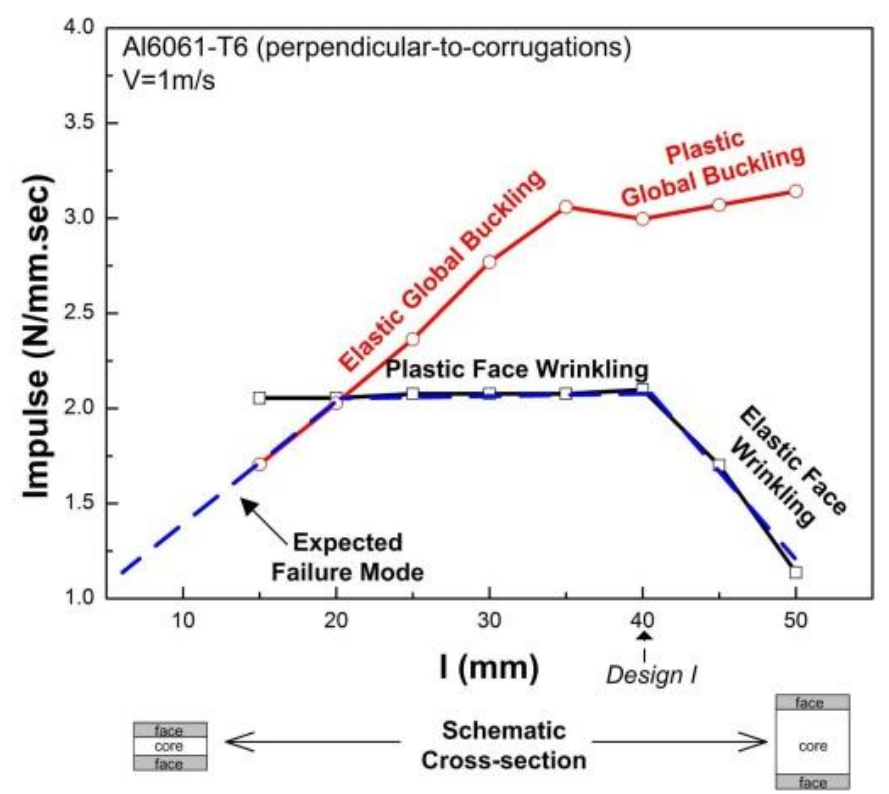

Figure 6.3: Competing failure modes of Al6061-T6 sandwich columns compressed perpendicular-tocorrugations.

Design I, is expected to fail by face wrinkling because the impulse calculated from the face wrinkling analytical model is smaller than the one from the global buckling one. The core height-varied designs whose core heights are greater than the one of Design I are expected to fail by elastic face wrinkling (EFW).

On the other hand, plastic face wrinkling failure dominates in the designs whose corrugation length, $\ell$, ranges from 20 to $40 \mathrm{~mm}$ at $\mathrm{V}=1 \mathrm{~m} / \mathrm{s}$. However, under quasi-static loading, the core height-varied designs in this range $(20<\ell<40)$ would fail by global buckling because their global static buckling strengths are smaller than that of Design I while their local static buckling strengths are larger than Design I.

In the smaller range of $\ell(\ell<20)$, Case_APerp17 and Case_APerp 18 are expected to fail by elastic global buckling.

Therefore, it is concluded that a smaller core-height design than the quasi-static optimal solution (Design I in this example) is allowable to maximize the impulse capacity 
under dynamic loading. In other words, Figure 6.3 implicitly shows that the dynamic optimal solutions are placed from the quasi-static solution to the direction of increasing local-buckling-strength and decreasing global buckling strength. (Remember that as $\ell$ decreases, the direction is toward increasing local buckling strength, at the same time, decreasing global buckling strength.)

Next, consider A16061-T6 corrugated core sandwich columns compressed parallelto-corrugations at $\mathrm{V}=1 \mathrm{~m} / \mathrm{s}$. Five core-height-varied designs from Design II are listed in Table 6.2. Likewise in Design I and its mass-equivalent and core-height varied designs, they have the same mass, corrugation angle, w, and face sheet thickness, h. As the core height-varied designs listed in Table 6.2 have smaller corrugation lengths, $\ell$, than that of Design II, the core height-varied designs have smaller global static buckling strengths but larger local static buckling strengths than Design II. As a result, all the listed designs would fail by global buckling under quasi-static loading.

Table 6.2. Core-height-varied designs of Design II.

\begin{tabular}{cccccc}
\hline Design No. & $\mathrm{h}$ & $\mathrm{t}$ & $\mathrm{l}$ & $\mathrm{w}$ & $\mathrm{M} /(\mathbf{\rho b L})$ \\
\hline \hline Case_Apara_Base (Design II) & $\mathbf{0 . 8 3 0 3}$ & $\mathbf{0 . 7 9 5 4}$ & $\mathbf{3 0 . 9 7}$ & $\mathbf{5 8 . 5 4}$ & $\mathbf{3 . 1 0}$ \\
Case_APara21 & 0.83 & 0.864163 & 10 & 60 & 3.11 \\
Case_APara22 & 0.83 & 0.810262 & 15 & 60 & 3.11 \\
Case_APara23 & 0.83 & 0.785756 & 20 & 60 & 3.11 \\
Case_APara24 & 0.83 & 0.771752 & 25 & 60 & 3.11 \\
Case_APara25* $^{*}$ & 0.83 & 0.76269 & 30 & 60 & 3.11 \\
\hline
\end{tabular}

* Case_Apara25 is the closest to Design II. 


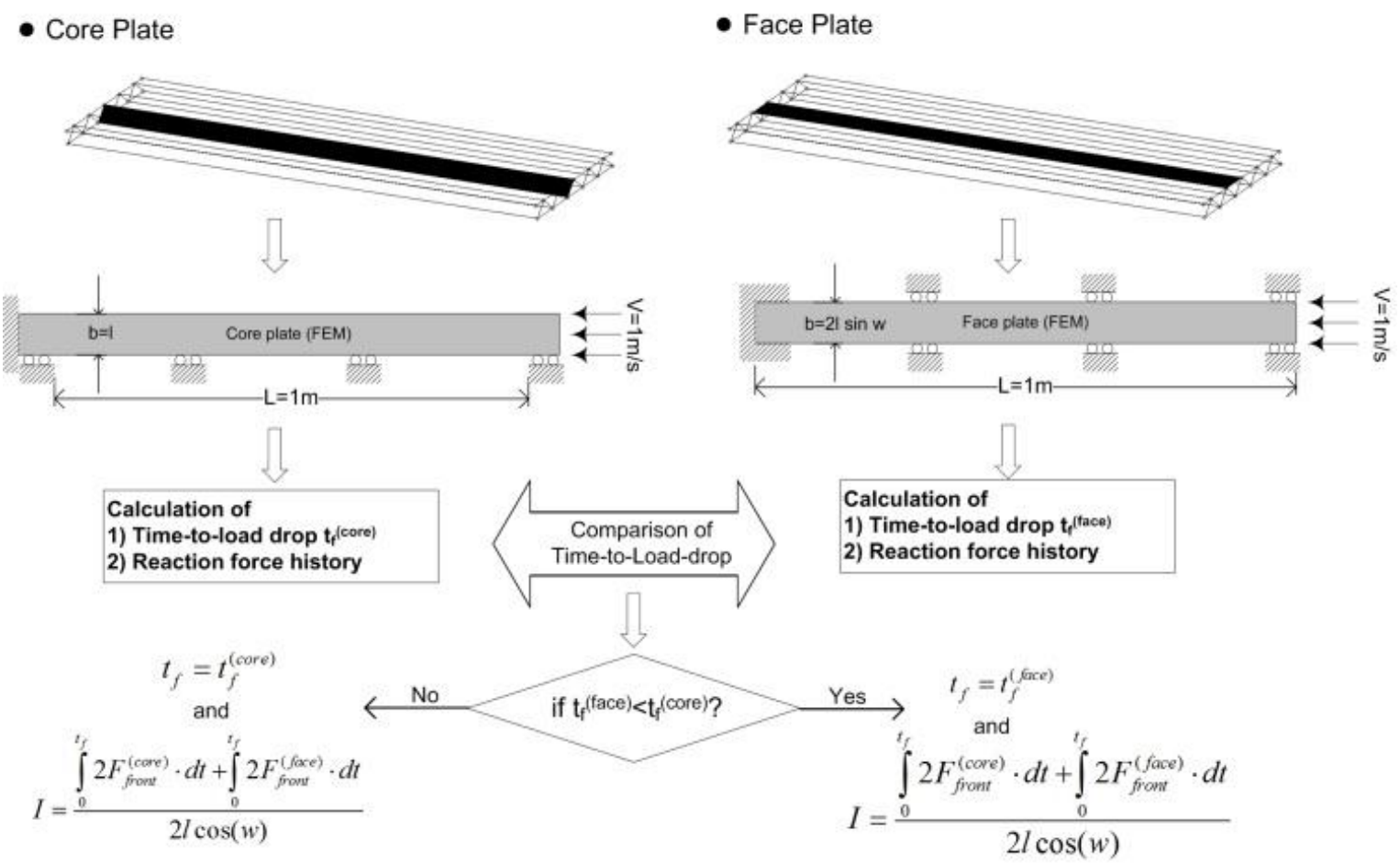

Figure 6.4: FE impulse capacity calculation of a sandwich column failed by local plate buckling.

The designs are tested at $\mathrm{V}=1 \mathrm{~m} / \mathrm{s}$. The impulse of global buckling at $\mathrm{V}=1 \mathrm{~m} / \mathrm{s}$ is obtained from the global buckling analytical model developed in Chapter 2. Global curvature imperfections are assigned in the same way as in Design $I$ cases; $\mathrm{w}_{0}(x)=$ $\frac{\xi^{(g)}}{2}\left(1-\cos \left(\frac{2 \pi x}{L}\right)\right)$ with $\xi^{(g)} / L=0.002$. The impulse calculation for local plate buckling has been done via FE simulations since the accuracy of the analytical approach for the failure mode (in Chapter 4) cannot be guaranteed. Therefore, two FE calculations per each design are performed for the plates simulating a core plate or a face plate. Imperfections in the form of the first eigenmode with a magnitude of one hundredth of their thickness (i.e., $\xi_{0}^{(L P)}=0.01 \mathrm{~h}$ for face plates, and $\xi_{0}^{(L P)}=0.01 \mathrm{t}$ for core plates) was included in the analysis. The FE impulse calculation procedure for local plate buckling is described in Fig. 6.4, and is used to estimate the impulse capacity of the sandwich design. 


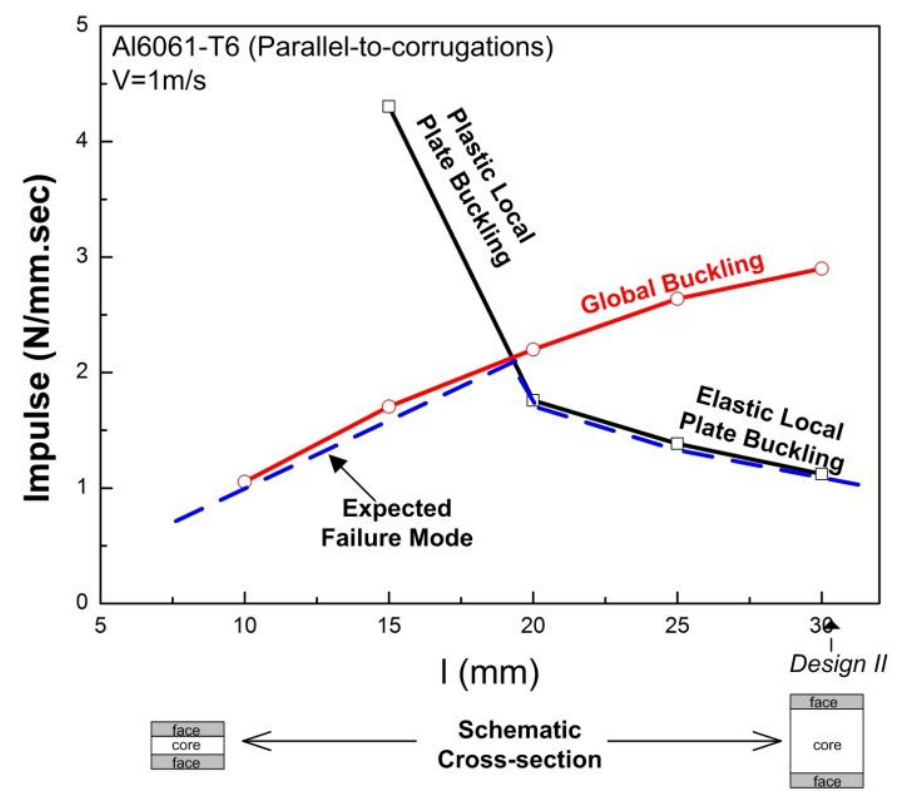

Figure 6.5: Competing failure modes of Al6061-T6 sandwich columns compressed parallel-tocorrugations under $\mathrm{V}=1 \mathrm{~m} / \mathrm{s}$.

The dominant failure mode (core plate or face plate) is the one with the lower impulse at time-to-load drop, as described in Fig. 6.4 and Eqn. (6.5).

$$
I=\frac{\int_{0}^{t_{f}} 2 F_{\text {front }}^{(\text {core })} \cdot d t+\int_{0}^{t_{f}} 2 F_{\text {front }}^{(\text {face })} \cdot d t}{2 l \cos (w)}
$$

Fig. 6.5 describes the competition of failure mechanisms for columns compressed parallel-to-corrugations made from Al6061-T6. As the core height increases, the impulse calculated from the global buckling analytical approach increases while the one obtained from local plate buckling FEA decreases. The blue dash line represents the maximum impulse capacity of each sandwich design at $\mathrm{V}=1 \mathrm{~m} / \mathrm{s}$. In addition, the line indicates the failure mode of each sandwich column design, based on smaller predicted impulse. As seen in the Fig. 6.5, the sandwich design having the maximum impulse capacity at $\mathrm{V}=1 \mathrm{~m} / \mathrm{s}$ is positioned between Case_Apara22 and Case_Apara23, and the quasi-static optimal solution (Design II) fails by local plate buckling if employed under dynamic 
loading. However, note that all the listed core-height varied designs in Table 6.2 would fail by global buckling under quasi-static loading.

Next, consider SS304 corrugated core sandwich columns compressed perpendicular-to-corrugations at two velocities, $\mathrm{V}=0.5 \mathrm{~m} / \mathrm{s}$ and $1 \mathrm{~m} / \mathrm{s}$. In order to investigate how the competing failure mechanisms evolve, the analysis has been carried out for these two velocities.

Table 6.3 shows eight different core height designs, varied around Design III. The core-height varied design whose $\ell$ is greater than that of Design III has a greater global static buckling strength but smaller local static buckling strength than Design III. On the contrary, the core height varied design whose $\ell$ is smaller than that of Design III has a smaller global static buckling strength but greater local static buckling strength.

As in the analysis of Design I (A16061-T6 sandwich columns in the perpendicularto-corrugations direction) and its mass-equivalent core-height-varied designs, the face wrinkling and global buckling analytical models are employed for impulse calculations

Table 6.3. Core-height-varied designs of Design III.

\begin{tabular}{cccccc}
\hline Design No, & $\mathrm{h}$ & $\mathrm{t}$ & $\mathrm{l}$ & $\mathrm{w}$ & $\mathrm{M} /(\mathbf{\rho b L})$ \\
\hline \hline Case_Sperp_Base (Design III) & $\mathbf{0 . 7 9 6 5}$ & $\mathbf{0 . 0 6 3}$ & $\mathbf{3 2 . 7 5}$ & $\mathbf{5 1 . 1 8}$ & $\mathbf{1 . 6 0}$ \\
Case_SPerp31 & 0.8 & 0.0063 & 40 & 52 & 1.61 \\
Case_SPerp32 & 0.8 & 0.0063 & 35 & 52 & 1.61 \\
Case_SPerp33 & 0.8 & 0.0063 & 30 & 52 & 1.61 \\
Case_SPerp34 & 0.8 & 0.0063 & 25 & 52 & 1.61 \\
Case_SPerp35 & 0.8 & 0.0063 & 20 & 52 & 1.61 \\
Case_SPerp36 & 0.8 & 0.0063 & 15 & 52 & 1.61 \\
Case_SPerp37 & 0.8 & 0.0063 & 10 & 52 & 1.61 \\
Case_SPerp38 & 0.8 & 0.0063 & 5 & 52 & 1.61 \\
\hline
\end{tabular}

* Case_Sperp33 is the closest to Design III. 
under dynamic loading. In addition, the assigned imperfections are $\mathrm{w}_{0}(x)=\frac{\xi^{(g)}}{2}(1-$ $\left.\cos \left(\frac{2 \pi x}{L}\right)\right)$ and $w_{0}^{(f)}=\frac{\xi^{(f)}}{2}\left(1-\cos \left(\frac{2 \pi x}{2 \ell \cos (\omega)}\right)\right)$ with magnitudes of $\xi^{(g)}=0.002 L$ $\xi^{(f)}=0.01 \mathrm{~h}$, for global curvature imperfections and face ligament curvature imperfections, respectively.

The results of the impulse calculations at the two velocities, $V=0.5 \mathrm{~m} / \mathrm{s}$ and $1 \mathrm{~m} / \mathrm{s}$ are shown in Fig 6.6. At $\mathrm{V}=0.5 \mathrm{~m} / \mathrm{s}$, the global buckling line and the face wrinkling line intersects around $\ell=8 \mathrm{~mm}$ as shown in Fig. 6.6(a). However, face wrinkling failure dominates at $\mathrm{V}=1 \mathrm{~m} / \mathrm{s}$ over all the considered designs at $\mathrm{V}=1 \mathrm{~m} / \mathrm{s}$. In effect, the global buckling line moves from the right of $\mathrm{x}$-axis to the left as $\mathrm{V}$ increases due to the inertial stabilization of global buckling motion. Face wrinkling dominates the failure of the columns as the applied velocity increases. 


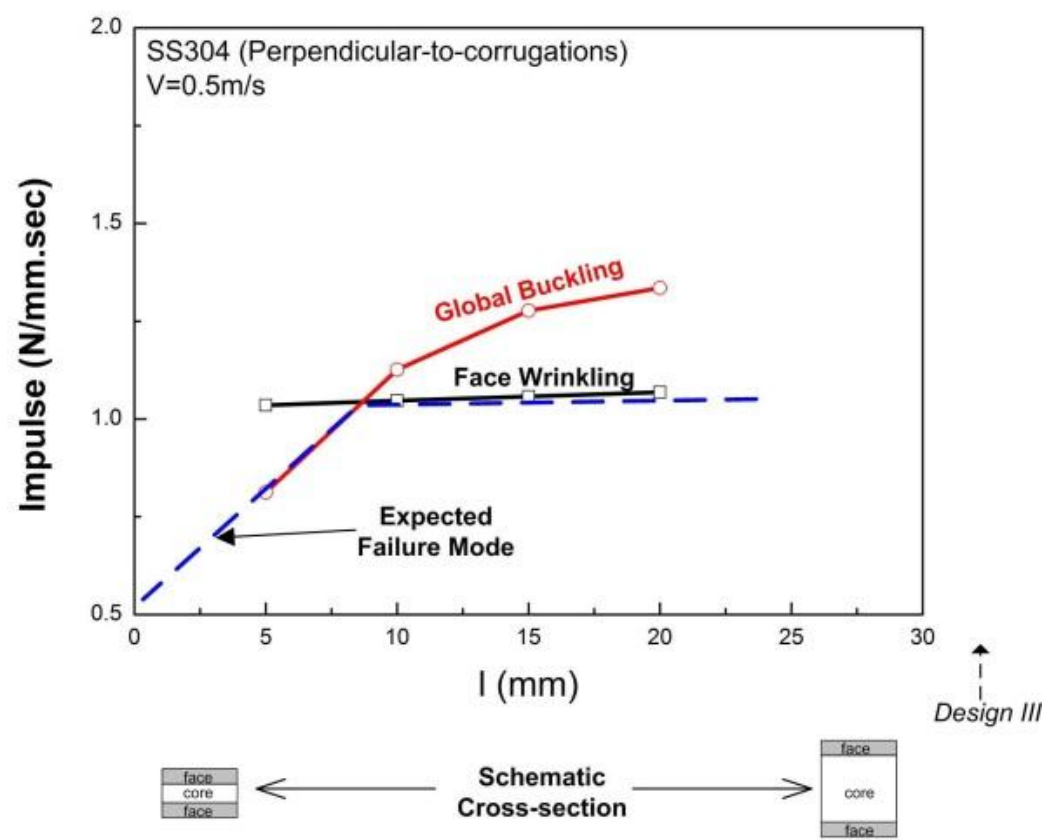

(a)

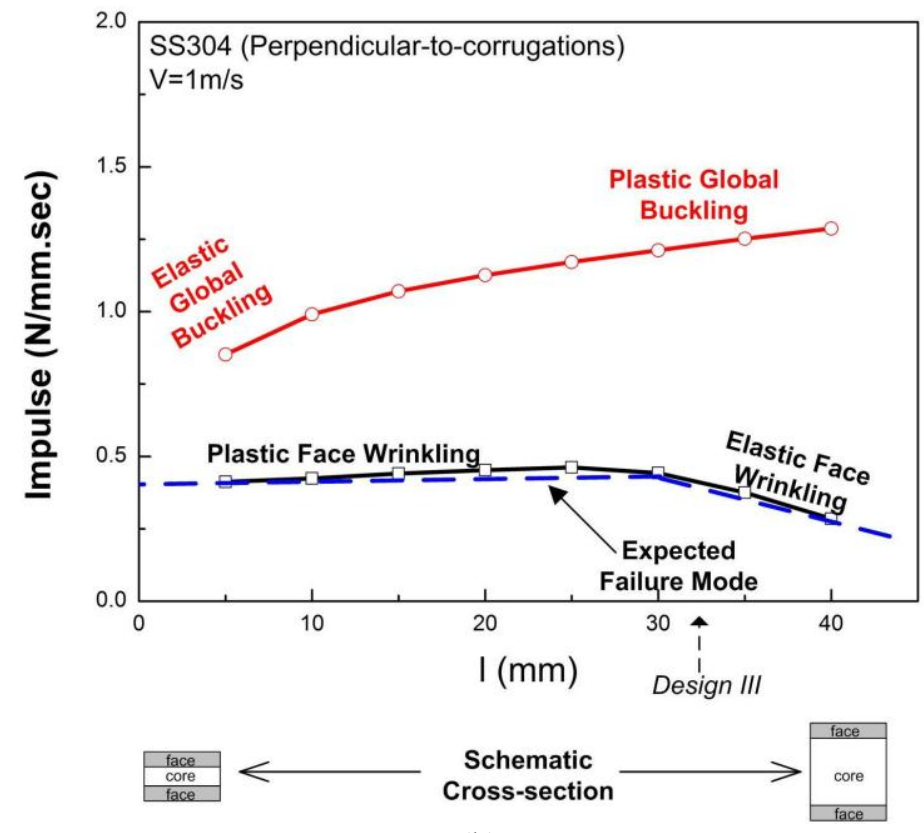

(b)

Figure 6.6: Competing failure modes of SS304 sandwich columns compressed perpendicular-tocorrugations under (a) $\mathrm{V}=0.5 \mathrm{~m} / \mathrm{s}$ and (b) $\mathrm{V}=1 \mathrm{~m} / \mathrm{s}$. 


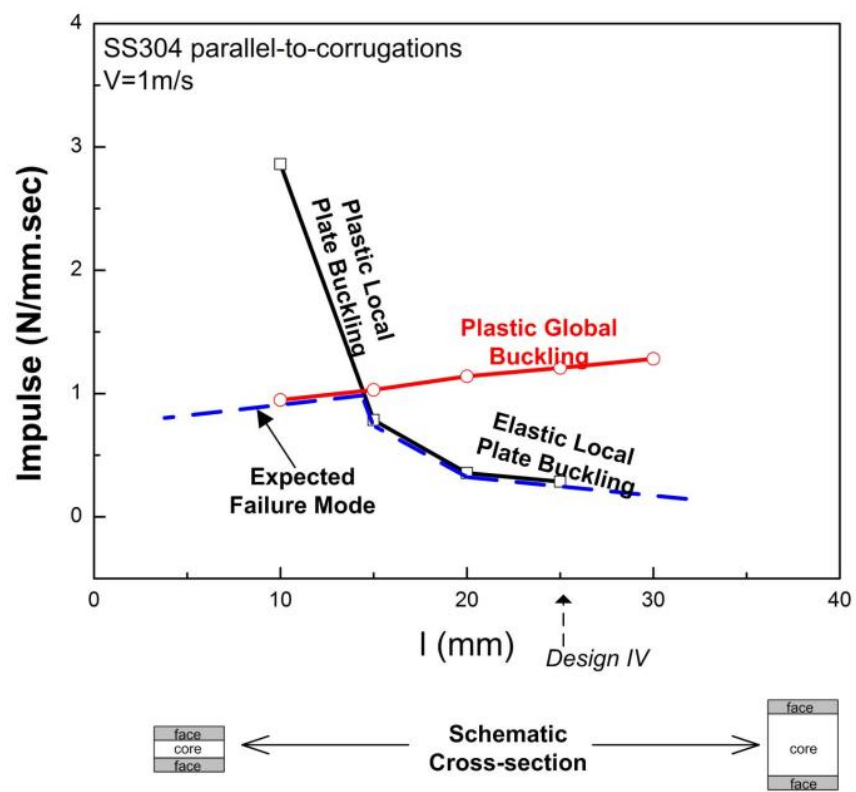

Figure 6.7: Competing failure modes of SS304 sandwich columns compressed parallel-to-corrugations under $\mathrm{V}=1 \mathrm{~m} / \mathrm{s}$.

Similarly, the maximum dynamic performance is also achieved by decreasing the core height of Design IV, which is the quasi-static optimal solution of SS304 corrugated core sandwich columns compressed parallel-to-corrugations as seen in Fig. 6.7. As in the other analyses, the design closest to the quasi-static optimal design, Case_Spara43 specified in Table 6.4 , fails by local buckling at $\mathrm{V}=1 \mathrm{~m} / \mathrm{s}$. By decreasing the core height (i.e., the enhancement of local plate buckling strength), sandwich columns in the direction of parallel-to-corrugations are caused to fail by simultaneous activation of the

Table 6.4. Core-height-varied designs of Design IV.

\begin{tabular}{cccccc}
\hline Design No. & $\mathrm{h}$ & $\mathrm{t}$ & $\mathrm{l}$ & $\mathrm{w}$ & $\mathrm{M} /(\mathbf{\rho b L})$ \\
\hline \hline Case_Spara_Base $($ Design $\boldsymbol{I} \boldsymbol{V})$ & $\mathbf{0 . 6}$ & $\mathbf{0 . 3 5}$ & $\mathbf{2 3 . 7 9}$ & $\mathbf{3 2 . 2 9}$ & $\mathbf{1 . 5 9}$ \\
Case_SPara41 & 0.6 & 0.35 & 10 & 30 & 1.61 \\
Case_SPara42 $^{\text {Case_SPara43* }}$ & 0.6 & 0.35 & 15 & 30 & 1.61 \\
Case_SPara44 $^{\text {Case_SPara45 }}$ & 0.6 & 0.35 & 20 & 30 & 1.61 \\
& 0.6 & 0.35 & 25 & 30 & 1.61 \\
\hline
\end{tabular}

* Case_Spara43 is the closest to Design IV. 
two failure modes.

In summary, the quasi-static optimal designs were tested under dynamic conditions. The increase in loading rate can cause a transition in failure mechanism. The structural columns to support dynamic in-plane loading should be designed such that local buckling strength is reinforced in order to sustain maximum impulse with minimized weight. This suggestion will be confirmed by the other methodology described in Section 6.4.

\subsection{DYNAMIC OPTIMIZATION FOR AL6061-T6 SANDWICH COLUMNS}

In this section, the optimal designs for Al6061-T6 corrugated core sandwich columns of $\mathrm{L}=1 \mathrm{~m}$ subject to dynamic compressive loads are studied. In particular, the investigation will focus on how minimum weight design change under increased rate-ofcompression from quasi-static loading to $\mathrm{V}=0.1$ and $1.0 \mathrm{~m} / \mathrm{s}$.

As previously mentioned, the dynamic response of sandwich columns is difficult to express with an explicit function of sandwich design variables. In this regard, the response of interest must be limited and approximated with other alternative functions such as polynomials of independent variables. The approximation procedure is referred to as Response Surface Methodology (RSM) [92]. The basic concept of the RSM is that an approximated response, $\widetilde{g}_{l}(\vec{x})$, called the response surface, is employed for an optimization procedure rather than using a complex and unknown response, $g_{i}(\vec{x})$ as a function of design vector $\overrightarrow{\mathrm{x}}$ (a set of design variables). The relationship between $\widetilde{g}_{l}(\vec{x})$ and $g_{i}(\vec{x})$ are described as

$$
g_{i}(\vec{x})=\tilde{g}_{i}(\vec{x})+\varepsilon_{i}
$$


In Eqn. (6.6), $\varepsilon_{\mathrm{i}}$ is the residual error, which is difference between the exact response, $g_{i}(\vec{x})$, and the approximated response, $\tilde{g}_{i}(\vec{x})$, and typically analyzed by means of statistics.

The success of this kind of optimization is determined by the accuracy of response approximation rather than the performance of optimization algorithms so that the interested impulse range ${ }^{1}$ is limited to $0.5<\mathrm{I}<4.0(\mathrm{~N} / \mathrm{mm} . \mathrm{s})$ for $\mathrm{V}=1 \mathrm{~m} / \mathrm{s}$, and $5<\mathrm{I}<40(\mathrm{~N} / \mathrm{mm} . \mathrm{s})$ for $0.1 \mathrm{~m} / \mathrm{s}$. Moreover, geometric constraints of the design variables are also assigned as follows: $0.5<\mathrm{h}<3.0 \mathrm{~mm}, 0.5<\mathrm{t}<2.0 \mathrm{~mm}, 0<\ell<100 \mathrm{~mm}, 30<\mathrm{w}<60^{\circ}$ for perpendicular-to-corrugations, and $0.5<\mathrm{h}, \mathrm{t}<2.0 \mathrm{~mm}, 0<\ell<100 \mathrm{~mm}, \mathrm{~h}=\mathrm{t}, \mathrm{w}=60^{\circ}$ for parallel-to-corrugations.

The analytical approaches developed in Chapters 2 and 3 are used for obtaining the dynamic response of individual failure modes except for the local plate buckling prediction of sandwich columns compressed parallel-to-corrugations. Instead, finite element analysis will be performed for this scenario as applied in Section 6.3. The first eigen-modes as geometric curvature imperfections for individual failure modes are assigned with a magnitude of $\xi_{g} / L=0.002$ for global buckling, $\xi^{(f)}=0.01 h$ for face wrinkling, or $\xi^{(l p)}=0.01 \mathrm{~h}$ or $0.01 t$ for local plate buckling, respectively. Commercial optimization software, Altair/Hyperstudy v10.0 [95], is employed for the optimization procedure in this section, which performs both the response surface methodology [92] and the optimization algorithm, SQP [93-94].

\footnotetext{
${ }^{1}$ It is impulse per unit plate width because reaction force is force per unit width.
} 


\subsubsection{Response Surface Methodology}

The complex dynamic response of Al6061-T6 corrugated core sandwich columns compressed perpendicular-to-corrugations and parallel-to-corrugations is given explicitly in terms of sandwich design variables through the RSM for compression velocities $\mathrm{V}=0.1$ and $1.0 \mathrm{~m} / \mathrm{s}$. As a result, polynomial functions which will be only valid in a limited design domain, will be representatives of the dynamic response. To differentiate the approximation with explicit functions (polynomial representation) from obtaining approximated responses by analytical approaches, the former will be called as 'response approximation.,'

Consider a global buckling response approximation of corrugated core sandwich columns compressed perpendicular-to-corrugations under $\mathrm{V}=1 \mathrm{~m} / \mathrm{s}$. In Table D1 of Appendix D, a full factorial experimental design for the response approximation is given, in which specimens with four face sheet thicknesses $(h=0.5,1,2,3 \mathrm{~mm})$, three corrugation thicknesses $(\mathrm{t}=0.5,1,2)$, ten corrugation lengths, $\ell$, and three corrugation angles $(\mathrm{w}=30$, $45,60)$ are tested, resulting in 360 numerical experiments.

For each numerical experiment, front end reaction force histories are calculated using the global buckling analytical approach. Subsequently, impulse is calculated by integrating the obtained reaction force history till time-to-load drop.

$$
I_{f r}=\int_{0}^{t_{f}} F_{f r} \cdot d t
$$

\footnotetext{
${ }^{1}$ Response approximation (a meta model); some called this kind of approximation as a meta model ('response approximation of approximated response') if the test results are obtained from numerical simulations such as FEM, analytical models etc.
} 


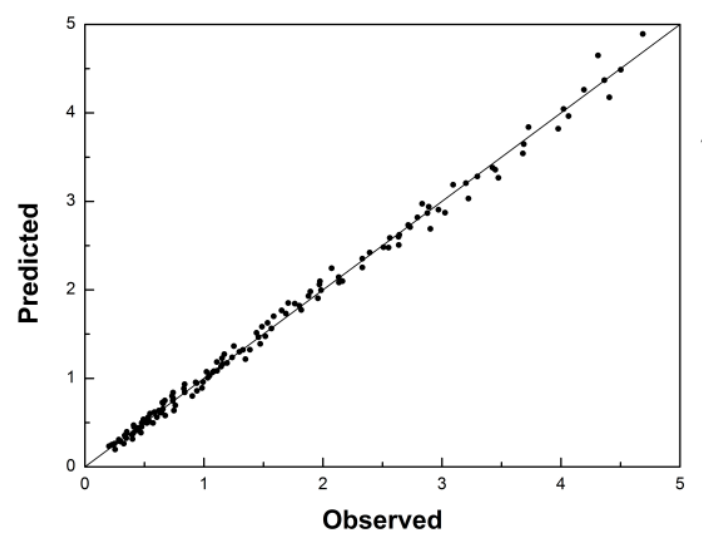

(a)

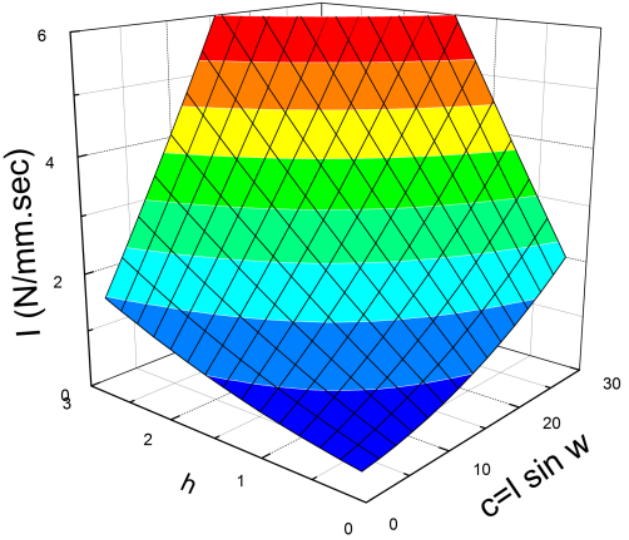

(b)

Figure 6.8: EGB (perpendicular-to-corrugations): (a) Residual plot and (b) Response surface for elastic global buckling response under $\mathrm{V}=1 \mathrm{~m} / \mathrm{s}(\mathrm{t}=1.0 \mathrm{~mm})$.

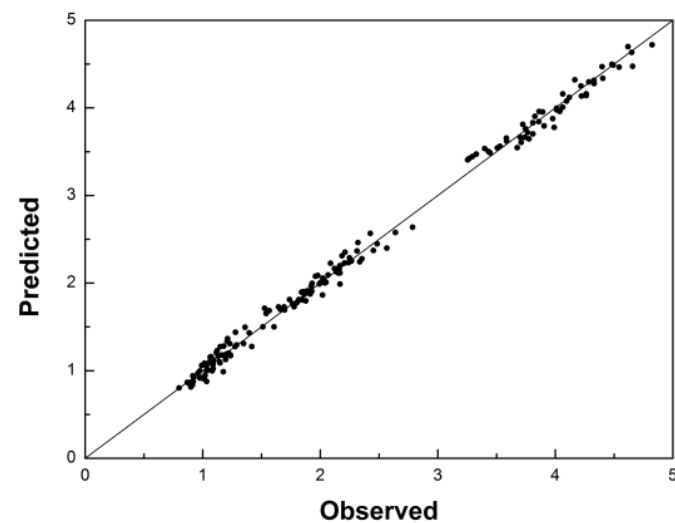

(a)

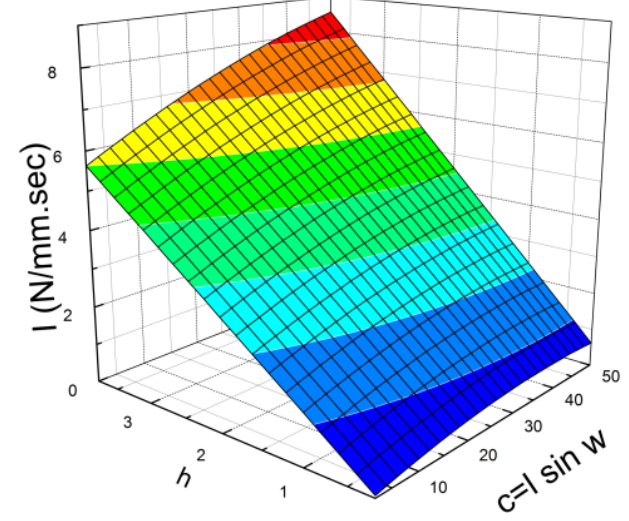

(b)

Figure 6.9: PGB (perpendicular-to-corrugations): (a) Residual plot and (b) Response surface for plastic global buckling response under $\mathrm{V}=1 \mathrm{~m} / \mathrm{s}(\mathrm{t}=1.0 \mathrm{~mm})$.

It should be noted that since the accuracy of the response approximation is critical, experiments whose responses are beyond the impulse range of interest are discarded for the response approximation. In addition, the response approximation is divided into elastic and plastic because the functions show an obvious distinction between the two. The method of least squares is used for parameter estimation.

The approximated polynomial functions of design variables for maximum impulse capacity are summarized in Equations (6.8), (6.9) for $\mathrm{V}=1 \mathrm{~m} / \mathrm{s}$, and (6.12), (6.13) for 
$\mathrm{V}=0.1 \mathrm{~m} / \mathrm{s}$. The resulting statistics are summarized in Table 6.5. A statistic, $\mathrm{R}^{2}$, is shown to be greater than 0.9 , which indicates high correlation between the approximated polynomials and the numerical experimental data. The residual plot and resulting response surface for the elastic and plastic global buckling motions under $\mathrm{V}=1 \mathrm{~m} / \mathrm{s}$ are shown in Fig.6.8 and Fig. 6.9, respectively. In the residual plots of Fig 6.8(a) and Fig.6.9(a), the vertical axis means the predicted impulse from the curve-fitted polynomials while the horizontal axis stands for the tested results (i.e., impulse calculations by analytical models). If the data points are placed near the line in each plot, the response approximation by polynomial can be said to be satisfactory.

A full factorial experimental design for impulse response approximation for face wrinkling failure mode of Al6061-T6 sandwich columns is given in Table D2 of Appendix D. The experimental design consists of a combination of four levels of $h(0.5,1$, $2,3)$, three levels of $\mathrm{t}(0.5,1,2)$, seven levels of $\ell$ and three levels of $\mathrm{w}(30,45,60)$. For the entire 252 experiments, impulse at the front end is calculated with the face-wrinkling analytical approach developed in Chapter 3. The resulting response approximations are given in Equations (6.10) and (6.11) for $\mathrm{V}=1 \mathrm{~m} / \mathrm{s}$, and Equations (6.14) and (6.15) for $\mathrm{V}=0.1 \mathrm{~m} / \mathrm{s}$. In addition, corresponding statistics are summarized in Table 6.5. Residual graphs and the face wrinkling response surfaces for $V=1 \mathrm{~m} / \mathrm{s}$ are illustrated in Figures 6.10 and 6.11 .

- Elastic global buckling under V=1.0m/s (Perpendicular-to-corrugations)

$$
\begin{aligned}
\tilde{g}_{1}^{(A l-p e r p)}(h, t, l, w)= & 0.1709586+(-0.0675246) \mathrm{h}+(0.0309833) \mathrm{t}+(-0.0399853) \ell \sin \mathrm{w} \\
& +(0.0841007) \mathrm{h}^{2}+(-0.0571254) \mathrm{t}^{2}+(0.0017178)(\ell \sin w)^{2} \\
& +(0.0563303) \mathrm{h} \cdot \mathrm{t}+(0.1035862) \mathrm{h} \cdot(\ell \sin w)+(0.0296650) \mathrm{t} \cdot(\ell \sin w)
\end{aligned}
$$




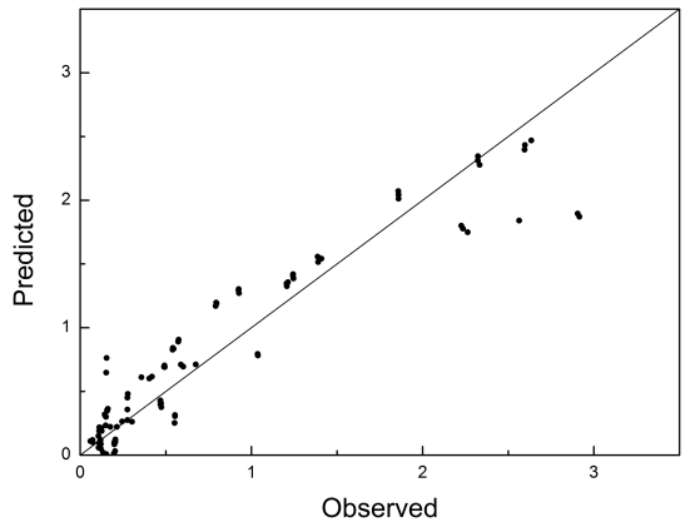

(a)

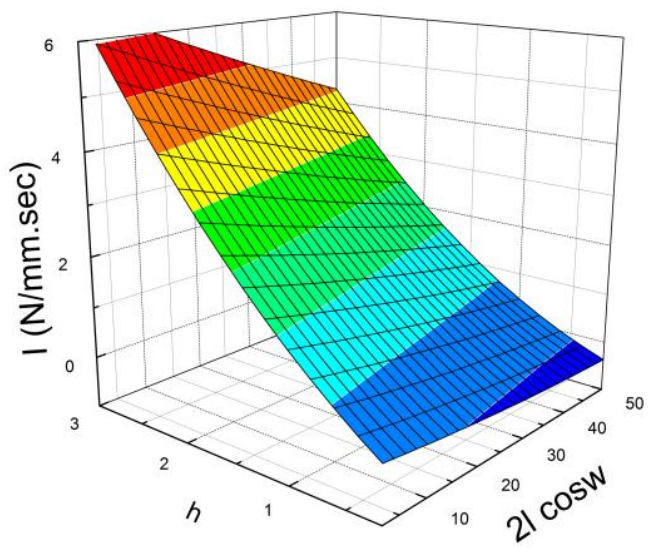

(b)

Figure 6.10: EFW (perpendicular-to-corrugations): (a) Residual plot, and (b) Response surface for elastic face wrinkling response under $\mathrm{V}=1 \mathrm{~m} / \mathrm{s}(\mathrm{t}=1.0 \mathrm{~mm})$.

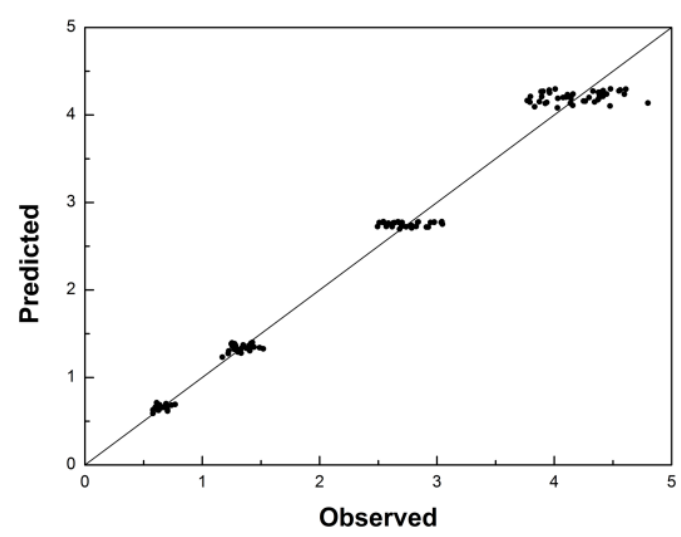

(a)

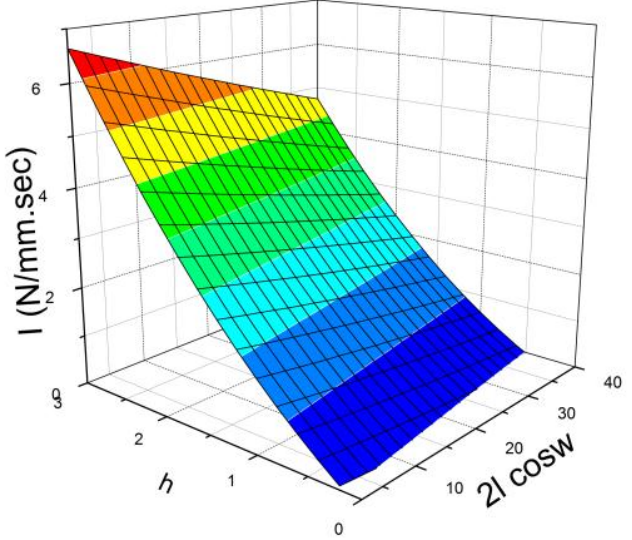

(b)

Figure 6.11: PFW(perpendicular-to-corrugations): (a) Residual plot and (b) Response surface for plastic face wrinkling response under $\mathrm{V}=1 \mathrm{~m} / \mathrm{s}(\mathrm{t}=1.0 \mathrm{~mm})$.

Plastic global buckling under V=1.0m/s (Perpendicular-to-corrugations)

$$
\begin{aligned}
\tilde{g}_{2}^{(A l-p e r p)}(h, t, l, w) & =-0.4228689+(1.5168608) \mathrm{h}+(0.1552485) \mathrm{t}+(0.0286442) \ell \sin \mathrm{w} \\
& +(-0.0062855) \mathrm{h}^{2}+(-0.0488618) \mathrm{t}^{2}+(-4.53 \mathrm{e}-04)(\ell \sin w)^{2}+ \\
& +(0.0271148) \mathrm{h} \cdot \mathrm{t}+(0.0136655) \mathrm{h} \cdot(\ell \sin w)+(0.0038931) \mathrm{t} \cdot(\ell \sin w)
\end{aligned}
$$

- Elastic face wrinkling under V=1.0m/s (Perpendicular-to-corrugations) 


$$
\begin{aligned}
\tilde{g}_{3}^{(A l-p e r p)}(h, t, l, w) & =-0.2046283+(1.3154471) \mathrm{h}+(0.0515360) \mathrm{t}+(-0.0119863)(2 \ell \cos w) \\
& +(0.3173782) \mathrm{h}^{2}+(-0.0236445) \mathrm{t}^{2}+(1.20 \mathrm{e}-04)(2 \ell \cos w)^{2} \\
& +(0.0408362) \mathrm{h} \cdot \mathrm{t}+(-0.0141695) \mathrm{h} \cdot(2 \ell \cos w)+(-6.66 \mathrm{e}-04) \mathrm{t} \cdot(2 \ell \cos w)
\end{aligned}
$$

- Plastic face wrinkling under V=1.0m/s (Perpendicular-to-corrugations)

$$
\begin{aligned}
\tilde{g}_{4}^{(A l-p e r p)}(h, t, l, w) & =0.1144946+(1.3885025) \mathrm{h}+(-0.2282694) \mathrm{t}+(-0.0077415)(2 \ell \cos \mathrm{w}) \\
& +(-0.0074789) \mathrm{h}^{2}+(0.0993416) \mathrm{t}^{2}+(-5.59 \mathrm{e}-05) \cdot(2 \ell \cos \mathrm{w})^{2}+ \\
& +(-0.0107762) \mathrm{h} \cdot \mathrm{t}+(0.0046224)(\mathrm{h}) \cdot(2 \ell \cos \mathrm{w})+(3.60 \mathrm{e}-04)(\mathrm{t}) \cdot(2 \ell \cos \mathrm{w})
\end{aligned}
$$

- Elastic global buckling under V=0.1m/s (Perpendicular-to-corrugations)

$$
\begin{aligned}
\tilde{g}_{5}^{(\text {Al-pepr })}(h, t, l, w) & =15.657571+(6.6978276) \mathrm{h}+(-8.4888258) \mathrm{t}+(-3.2840058) \ell \sin \mathrm{w} \\
& +(-4.1062569) \mathrm{h}^{2}+(2.9008331) \mathrm{t}^{2}+(0.2308017)(\ell \sin w)^{2}+(3.1547413) \mathrm{h} \cdot \mathrm{t} \\
& +(-1.1633736) \mathrm{h} \cdot(\ell \sin w)+(0.2967039) \mathrm{t} \cdot(\ell \sin w)+(0.4433579) \mathrm{h}^{3}+(-0.0055382)(\ell \sin w)^{3} \\
& +(-0.4729058) \mathrm{h}^{2} \cdot \mathrm{t}+(-0.3660887) \mathrm{h} \cdot \mathrm{t}^{2}
\end{aligned}
$$

- Plastic global buckling under V=0.1m/s (Perpendicular-to-corrugations)

$$
\tilde{g}_{6}^{(A l-p e r p)}(h, t, l, w)=-0.0521955+(14.483407) \mathrm{h}
$$

- Elastic face wrinkling under $\mathrm{V}=0.1 \mathrm{~m} / \mathrm{s}$ (Perpendicular-to-corrugations)

$$
\begin{aligned}
\tilde{g}_{7}^{(A l-p e r p)}(h, t, l, w) & =-4.3407479+(37.705770) \mathrm{h}+(0.6911561) \mathrm{t}+(-0.7046823)(2 \ell \cos w) \\
& +(-6.6876168) \mathrm{h}^{2}+(0.2503075) \mathrm{t}^{2}+(-0.0015621)(2 \ell \cos w)^{2} \\
& +(-6.1952847) \mathrm{h} \cdot \mathrm{t}+(0.2157633) \mathrm{h} \cdot(2 \ell \cos w)+(0.1276817) \mathrm{t} \cdot(2 \ell \cos w)
\end{aligned}
$$

- Plastic face wrinkling under V=0.1m/s (Perpendicular-to-corrugations)

$$
\tilde{g}_{8}^{(A l-p e r p)}(h, t, l, w)=-0.0150867+(13.691845) \mathrm{h}
$$

Regarding Al6061-T6 corrugated core sandwich columns compressed parallel-tocorrugations, two design variables, $\mathrm{h}, \ell$, are considered with constraints to reduce the number of experiments: $\omega=60^{\circ}, \mathrm{t}=\mathrm{h}$. For global buckling motion, seven levels of $\mathrm{h}(0.5$, 
$0.75,1,1.25,1.5,1.75,2)$, and ten levels of $\ell$ construct a full factorial design consisting of 70 experiments for $\mathrm{V}=0.1$, and $1 \mathrm{~m} / \mathrm{s}$ as given in Table D3 of Appendix D.

In the case of local plate buckling, a full factorial design of 21 numerical experiments for each velocity $\mathrm{V}=0.1$ and $1 \mathrm{~m} / \mathrm{s}$ is constructed as shown in Appendix D4. The experimental design consists of seven levels of $\mathrm{h}$ and three levels of $\ell$.

Impulses are obtained using the global buckling analytical approach, and FEM as detailed in Section 6.3. Similarly to the perpendicular-to-corrugations, the data which is impulse within the interested range, is collected and approximated with polynomials of design variables. The resulting approximated equations and statistics are summarized in Equations (6.16)-(6.21) and in Table 6.5.

- Elastic global buckling under V=1.0m/s (Parallel-to-corrugations)

$$
\begin{aligned}
\tilde{g}_{1}^{(A l-\text { para })}(h, l)= & 0.1956026+(-0.0997822) \mathrm{h}+(-0.0314052) \ell \\
& +(0.0695462) \mathrm{h}^{2}+(0.0011726) \ell^{2}+(0.1352505) \mathrm{h} \cdot \ell
\end{aligned}
$$

- Plastic global buckling under V=1.0m/s (Parallel-to-corrugations)

$$
\tilde{g}_{2}^{(A l-p a r a)}(h, l)=-0.5758818+(3.3465553) \mathrm{h}+(0.0172231) \ell
$$

- Local plate buckling under $\mathrm{V}=1.0 \mathrm{~m} / \mathrm{s}$ (Parallel-to-corrugations)

$$
\begin{aligned}
\tilde{g}_{3}^{(\text {Al-para })}(h, l) & =-14.390687+(26.957723) \mathrm{h}+(0.7920770) \ell \\
& +(2.5501371) \mathrm{h}^{2}+(-0.0100981) \ell^{2}+(-1.4786034) \mathrm{h} \cdot \ell+(0.0189604) \mathrm{h} \cdot \ell^{2}
\end{aligned}
$$

- Elastic global buckling under V=0.1m/s (Parallel-to-corrugations)

$$
\begin{aligned}
\tilde{g}_{4}^{(A l-p a r a)}(h, l)= & 1.9486206+(-35.140836) \mathrm{h}+(0.1078835) \ell \\
& +(7.0930762) \mathrm{h}^{2}+(0.0041762) \ell^{2}+(1.6196441) \mathrm{h} \cdot \ell
\end{aligned}
$$

- Plastic global buckling under V=0.1m/s (Parallel-to-corrugations)

$$
\tilde{g}_{5}^{(\text {Al-para })}(h, l)=-0.1579522+(24.441435) \mathrm{h}+(0.0300818) \ell
$$


Table 6.5. Summary of statistics for the response surfaces.

\begin{tabular}{|c|c|c|c|c|c|c|}
\hline $\begin{array}{l}\text { In-plane } \\
\text { loading } \\
\text { orientation }\end{array}$ & $\begin{array}{c}\mathrm{V} \\
(\mathrm{m} / \mathrm{s})\end{array}$ & $\begin{array}{l}\text { Failure } \\
\text { mode }\end{array}$ & Design Parameters & $\begin{array}{c}\text { Order of } \\
\text { Polynomial }\end{array}$ & $\mathrm{R}^{2}$ & $\begin{array}{c}\text { \# of } \\
\text { experiments } \\
\text { used for } \\
\text { RSM }\end{array}$ \\
\hline \multirow{8}{*}{$\begin{array}{c}\text { Perpendicular- } \\
\text { to- } \\
\text { corrugations }\end{array}$} & \multirow{4}{*}{1.0} & EGB & $\mathrm{h}, \mathrm{t}, \ell \sin (\mathrm{w})$ & 2 & 0.9953199 & 135 \\
\hline & & PGB & $\mathrm{h}, \mathrm{t}, \ell \sin (\mathrm{w})$ & 2 & 0.9970090 & 224 \\
\hline & & EFW & $\mathrm{h}, \mathrm{t}, \quad 2 \ell \cos (\mathrm{w})$ & 2 & 0.8846610 & 113 \\
\hline & & PFW & $\mathrm{h}, \mathrm{t}, 2 \ell \cos (\mathrm{w})$ & 2 & 0.9817564 & 112 \\
\hline & \multirow{4}{*}{0.1} & EGB & $\mathrm{h}, \mathrm{t}, \ell \sin (\mathrm{w})$ & 3 & 0.9689700 & 146 \\
\hline & & PGB & $\mathrm{h}, \mathrm{t}, \ell \sin (\mathrm{w})$ & 1 & 0.9978398 & 113 \\
\hline & & EFW & $\mathrm{h}, \mathrm{t}, 2 \ell \cos (\mathrm{w})$ & 2 & 0.8673762 & 72 \\
\hline & & PFW & $\mathrm{h}, \mathrm{t}, 2 \ell \cos (\mathrm{w})$ & 1 & 0.9997984 & 99 \\
\hline \multirow{6}{*}{$\begin{array}{l}\text { Parallel-to- } \\
\text { corrugations }\end{array}$} & \multirow{3}{*}{1.0} & EGB & $\mathrm{h}, \mathrm{l}$ & 2 & 0.9959884 & 32 \\
\hline & & PGB & $\mathrm{h}, \ell$ & 1 & 0.9947609 & 20 \\
\hline & & LPB & $\mathrm{h}, \mathrm{l}$ & 3 & 0.9652911 & 12 \\
\hline & \multirow{3}{*}{0.1} & EGB & $\mathrm{h}, \mathrm{l}$ & 2 & 0.9736527 & 20 \\
\hline & & PGB & $\mathrm{h}, \mathrm{l}$ & 1 & 0.9994376 & 21 \\
\hline & & LPB & $\mathrm{h}, \mathrm{l}$ & 3 & 0.9865088 & 12 \\
\hline
\end{tabular}

- Local core plate buckling under $\mathrm{V}=0.1 \mathrm{~m} / \mathrm{s}$ (Parallel-to-corrugations)

$$
\begin{aligned}
\tilde{g}_{6}^{(A l-p a r a)}(h, l)= & -109.28181+(94.619872) \mathrm{h}+(9.7964445) \ell+(195.60548) \mathrm{h}^{2}+(-0.1763206) \ell^{2} \\
& +(-14.434154) \mathrm{h} \cdot \ell+(-4.5730210) \mathrm{h}^{2} \cdot \ell+(0.3048533) \mathrm{h} \cdot \ell^{2}
\end{aligned}
$$

\subsubsection{Optimization of Al6061-T6 Sandwich Columns under Dynamic In-}

\section{plane Loading}

Based on the approximated dynamic response, minimum weight designs of Al6061-

T6 corrugated core sandwich columns for sustaining a given impulse are obtained by employing the SQP. Impulse of interest is predefined as $0.5<\mathrm{I}<4(\mathrm{~N} / \mathrm{mm}$.sec) for $\mathrm{V}=1 \mathrm{~m} / \mathrm{s}$, and $5<\mathrm{I}<40(\mathrm{~N} / \mathrm{mm} . \mathrm{sec})$ for $\mathrm{V}=0.1 \mathrm{~m} / \mathrm{s}$ as mentioned previously. Geometric constraints are assigned additionally as follows: $0.5<\mathrm{h}<3(\mathrm{~mm}), \quad 0.5<\mathrm{t}<2(\mathrm{~mm}), \quad \ell<100(\mathrm{~mm})$, and $30^{\circ}<\mathrm{w}<60^{\circ}$ for perpendicular-to-corrugations and $0.5<\mathrm{h}<2(\mathrm{~mm}), \quad \ell<100(\mathrm{~mm}), \mathrm{t}=\mathrm{h}$, $\mathrm{w}=60^{\circ}$ for parallel-to-corrugations. Consequently, the optimization problems can be 
formulated in Equations (6.22) and (6.23) for the in-plane loading orientations of perpendicular-to-corrugation and parallel-to-corrugations, respectively.

- Optimization problem of Al6061-T6 corrugated core sandwich columns dynamically loaded perpendicular-to-corrugations

$$
\text { To minimize } f^{(A l-p e r p)}(h, l, t, w)=2 b L \rho\left(h+\frac{t}{2 \cos (w)+\frac{2 t}{l \sin (w)}}\right)
$$

Subject to

$$
\begin{aligned}
& \tilde{g}_{i}^{(A l-p e r p)}(h, t, l, \varpi)>I_{\text {given }}, \quad \mathrm{i}=\mathrm{m}, \ldots \mathrm{n} \\
& 0.5<\mathrm{h}<3(\mathrm{~mm}) \\
& 0.5<\mathrm{t}<2(\mathrm{~mm}) \\
& 0<1<100(\mathrm{~mm}) \\
& 30<\mathrm{w}<60\left(^{\circ}\right)
\end{aligned}
$$

where $(m, n)=(1,4)$ for $V=1 \mathrm{~m} / \mathrm{s},(\mathrm{m}, \mathrm{n})=(5,8)$ for $\mathrm{V}=0.1 \mathrm{~m} / \mathrm{s}$

- Optimization problem of Al6061-T6 corrugated core sandwich columns dynamically loaded parallel-to-corrugations

$$
\text { To minimize } f^{(A l-p e r p)}(h, l, t, w)=2 b L \rho\left(h+\frac{t}{2 \cos (w)+\frac{2 t}{l \sin (w)}}\right)
$$

Subject to 


$$
\begin{aligned}
& \tilde{g}_{i}^{(A l-\text { para })}(h, t, l)>I_{\text {given }}, \quad \mathrm{i}=\mathrm{m}, \ldots \mathrm{n} \\
& 0.5<\mathrm{h}<3(\mathrm{~mm}) \\
& \mathrm{t}=\mathrm{h} \\
& 0<1<100(\mathrm{~mm}) \\
& \mathrm{W}=60\left(^{\circ}\right)
\end{aligned}
$$

where $(m, n)=(1,3)$ for $V=1 \mathrm{~m} / \mathrm{s},(\mathrm{m}, \mathrm{n})=(4,6)$ for $\mathrm{V}=0.1 \mathrm{~m} / \mathrm{s}$

In these equations, $\mathrm{f}$ represents mass, and $\tilde{\mathrm{g}}$ means the approximated impulse capacity of a sandwich column as a design constraint in the optimization problem. The four types of the optimization problems (two in-plane loading orientations and two compressive velocities) are solved via a commercial optimization software program, Altair/Hyperstudy v10.0 [95] employing the SQP algorithm [92-94].

The optimization results are plotted together with monolithic solid columns having the same global curvature definition in Fig. 6.12. Monolithic solid column response is also calculated using the analytical approach presented in Chapter 2.

As shown in Fig. 6.12, Al6061-T6 corrugated core sandwich columns under both in-plane loading orientations are superior to monolithic solid columns in that they sustain a given impulse with less weight $(M / \rho b L)$ than monolithic solid columns for the two velocities, $V=0.1$ and $1 \mathrm{~m} / \mathrm{s}$. However, beneficial sandwich effects are more remarkable for the lower compressive velocity $V=0.1 \mathrm{~m} / \mathrm{s}$ than for $\mathrm{V}=1 \mathrm{~m} / \mathrm{s}$. That is because monolithic solid column structures are also influenced by inertial stabilization as compressive velocity increases $(\mathrm{V}=1 \mathrm{~m} / \mathrm{s})$.

The reason that sandwich columns loaded parallel-to-corrugations are always 


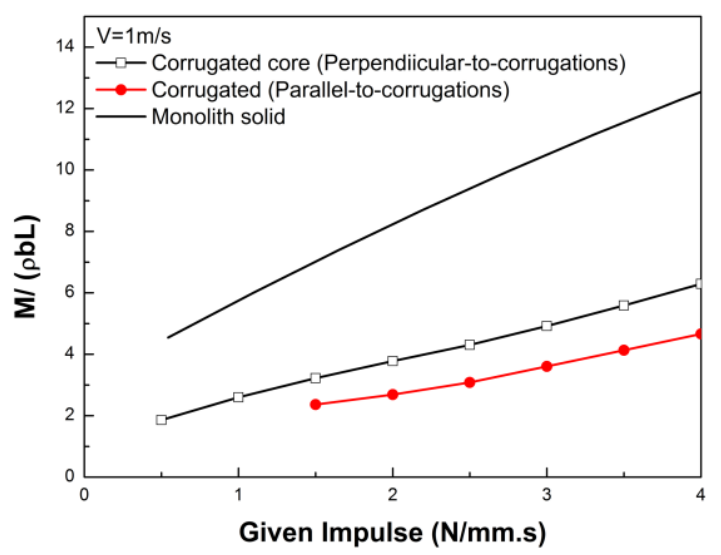

(a)

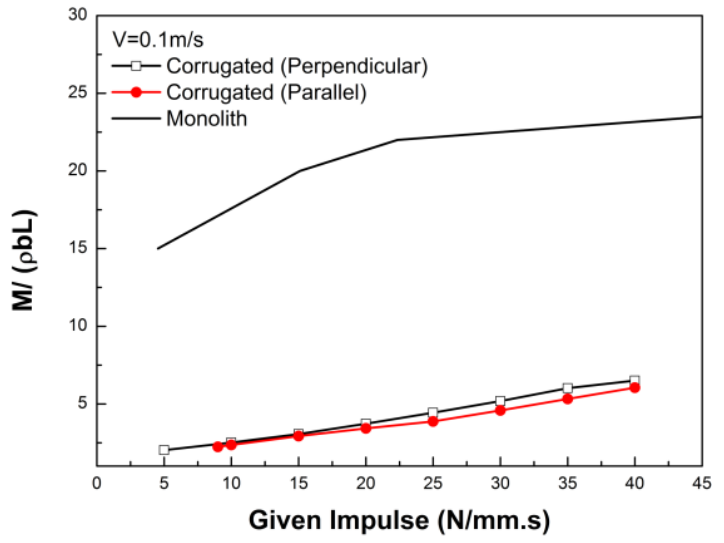

(b)

Figure 6.12: Comparisons of minimum weight designs of Al6061 corrugated core sandwich columns under (a) $\mathrm{V}=1 \mathrm{~m} / \mathrm{s}$ and (b) $\mathrm{V}=0.1 \mathrm{~m} / \mathrm{s}$.

superior to perpendicular-to-corrugations unlike the quasi-static optimization results in Section 6.2 is because of the additional constraint definition in this dynamic optimization problem, $\mathrm{t}_{\mathrm{min}}=0.5$. That is, the dimension of $\mathrm{t}$ may not be practical if there is no minimum constraint as in the quasi-static optimization problem in Section 6.2.

Fig. 6.13 shows the optimal cross-sectional shape of sandwich columns compressed perpendicular-to-corrugations depending on the compressive velocities.

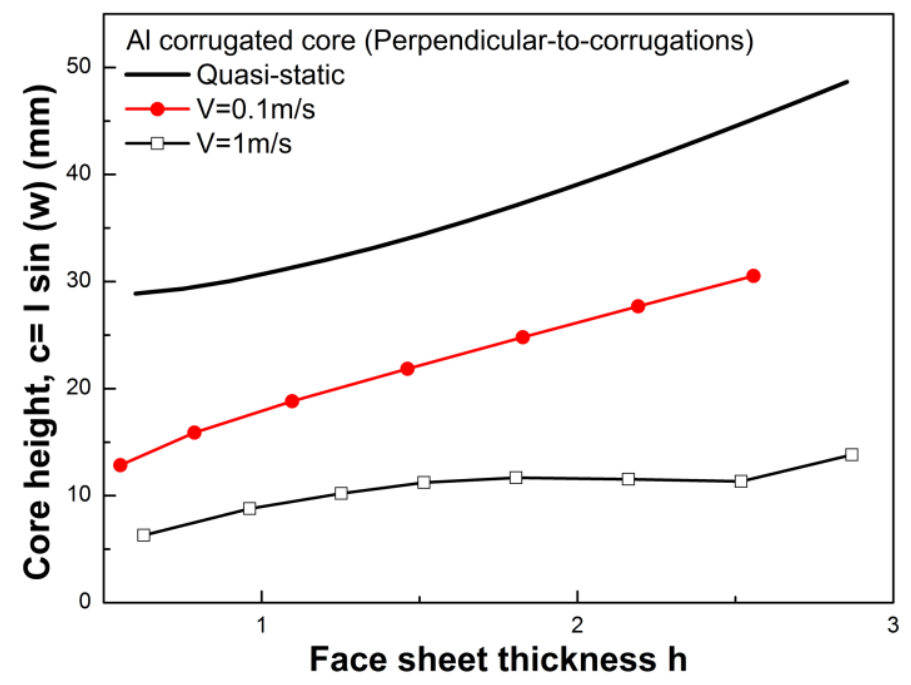

Figure 6.13: Variation of optimal core height for face sheet thickness h of optimal design for Al6061T6 sandwich columns compressed perpendicular-to-corrugations. 


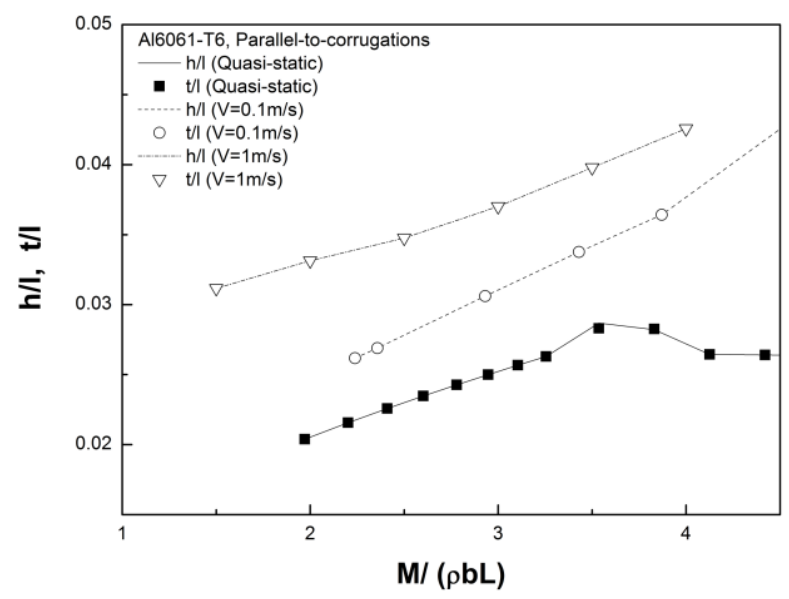

Figure 6.14: Local buckling strength, h/l and t/l for the same aerial mass of Al6061T6 sandwich columns compressed parallel-to-corrugations under quasi-static loading, $\mathrm{V}=1 \mathrm{~m} / \mathrm{s}$ and $\mathrm{V}=0.1 \mathrm{~m} / \mathrm{s}$.

Optimal core height, $\mathrm{c}=\ell \cdot \sin \omega$, for the same face sheet thickness tends to decrease as rate-of-loading increases. This result explains that the global buckling motion is influenced favorably by inertial stabilization more than the face wrinkling failure mode. This phenomenon is consistent with the observations in both Chapter 5 and Section 6.3. (Remember that maximized impulse capacity is found in sandwich columns whose core height is less than quasi-static optimal solution in the analysis of core-height varied design in Section 6.3)

Fig. 6.14 shows the evolution of optimal design variables in Al6061-T6 corrugated core sandwich columns compressed parallel-to-corrugations depending on rate-ofloading. As the rate-of-loading increases, the optimal ratios associated with local plate buckling strength, $h / \ell$ and $t / \ell$, increase for the same effective cross sectional area ${ }^{1}$, $A_{e f f}=(2 h+\bar{\rho} c)=\frac{M}{\rho b L}$, to achieve a given structural performance (i.e. sustaining a given

${ }^{1}$ aerial mass if expressed differently 
impulse in this study): face sheet thickness-to-corrugation length ratio $(h / \ell)$ and corrugation thickness to corrugation length ratio $(t / \ell)$. This phenomenon is also consistent with the observations in Section 6.3. (Remember that maximized impulse capacity is found in sandwich columns whose core height is less than quasi-static optimal solution in the analysis of core-height varied design in Section 6.3)

\subsection{CHAPTER SUMMARY}

It is shown that optimal designs of corrugated core sandwich columns under quasistatic loading and under dynamic loading are different. Although the optimization procedure in this chapter focuses on the specific range of impulse and assumed imperfections, the study in this chapter is informative in that:

(1) Global buckling motion is influenced more by beneficial inertial stabilization than the face wrinkling motion.

(2) As compression velocity is increased, minimum weight designs of corrugated core sandwich columns should reinforce local buckling strength in order to sustain a given impulse.

(3) Sandwich columns are superior to monolithic solid columns under dynamic loading; however, sandwich effects are more remarkable at lower applied velocities.

(4) A methodology for corrugated core sandwich columns is provided in the case where there is no explicit expression for dynamic response with design variables. 


\section{CHAPTER 7}

\section{CHARACTERIZATION OF HIGH VELOCITY RESPONSE}

\subsection{INTRODUCTION}

In this chapter, the dynamic response of corrugated core sandwich columns under high velocities is investigated through FEM, and characterized analytically.

When one end of a column is compressed at a compression velocity in the high

velocity range, $V /\left(c_{e l}^{*} \epsilon_{Y}\right)>1$ (Here, $c_{e l}^{*}$ can be either $c_{e l}^{(\text {Perp })}, c_{e l}^{(\text {Para })}$ or $\left.c_{e l}^{(m)}\right)$, the response is determined during the time period for a one-way trip of the plastic stress wave. This differs from the low velocity response discussed in previous chapters (Chap. 2 6), where many (at least a few) wave reflections have occurred until the column fails. Therefore, the high velocity sandwich column response can be parameterized in terms of sandwich geometric dimensions and loading intensity by applying the theory of rateindependent elastic-plastic wave propagation using an analogy between monolithic solid columns and sandwich columns. The range of applied compression velocity is considered up to $100 \mathrm{~m} / \mathrm{s}$, which front faces of typical sandwich panels attain during an underwater blast $[3,25]$.

The chapter is organized as follows: In Section 7.2, details of the FE model are described. In Section 7.3, the high velocity response for corrugated core sandwich columns compressed perpendicular-to-corrugations is investigated. Specifically, the effects of applied velocity and sandwich column geometric dimensions on reaction force are noted. The investigation is mainly focused on the sandwich columns made of a rate- 
independent material, A16061-T6, whose material strain-rate dependence can be neglected. However, the effect of material strain-rate dependence on the dynamic response is analyzed through FE simulations on SS304 corrugated core sandwich columns with and without consideration of the strain rate sensitivity of the parent material. In Section 7.4, the dynamic in-plane response in the parallel-to-corrugations direction is investigated.

\subsection{FEA}

Finite element simulations are performed based on material and loading direction: 1a) Al6061-T6 sandwich columns manufactured by extrusion/friction weld, 1b) SS304 sandwich columns manufactured by bending/brazing, 2a) perpendicular-to-corrugations, and 2b) parallel-to-corrugations.

A commercial FE package, ABAQUS/Explicit, is employed for the FE simulations. In-plane compression velocities in the range of $20 \sim 100 \mathrm{~m} / \mathrm{s}$ are imposed at one end of sandwich columns such that $V /\left(c_{e l}^{(S W)} \epsilon_{Y}\right)>1$. If the structural integrity of a column is damaged, or characteristic structural response is observed, calculations are interrupted and terminated. For example, when there is a loss of axial stability due to global buckling or progressive densification of unit cells observed in the perpendicular-to-corrugations models.

The details on elements, materials, boundary conditions are described in Chapter 2.5. 


\subsection{HIGH VELOCITY RESPONSE OF SANDWICH COLUMNS}

\section{(PERPENDICUALR-TO-CORRUGATIONS)}

The high velocity response of sandwich columns compressed perpendicular-tocorrugations is investigated. FE simulations are performed to obtain the response of the sandwich columns of varied geometric dimensions subject to compression velocities up to $100 \mathrm{~m} / \mathrm{s}$. Initial studies are performed using properties for a rate-independent (A16061T6) corrugated core sandwich columns. Simple analytic expressions incorporating general characteristics observed in the rate-independent FE analysis are suggested. The influence of material strain-rate dependence is assessed numerically for column manufactured from SS304.

\subsubsection{General Observations}

Representative dynamic response of corrugated core sandwich column compression perpendicular-to-corrugations is shown in Fig. 7.1. The plot represents the reaction force curves measured at the front and back ends of an Al6061-T6 sandwich column of $\mathrm{h}=2.2 \mathrm{~mm}, \mathrm{~L}=469 \mathrm{~mm}, \bar{\rho}=25 \%\left(\mathrm{t}=3.175 \mathrm{~mm}, \mathrm{l}=22 \mathrm{~mm}, \mathrm{w}=60^{\circ}\right)$ at $\mathrm{V}=60 \mathrm{~m} / \mathrm{s}$. The front end reaction force reveals an instantaneous rise after imposing the compressive velocity, followed by an immediate decay over $100 \mu \mathrm{s}$. While this observation at the front end is in progress, no response is detected at the back end. However, the back end reaction force rises to achieve a peak reaction force around $t=150 \mu \mathrm{s}$, remain at the peak for a period of time, and declines eventually. Also, additional increase in the front end reaction force is observed at $\mathrm{t}=400 \mu \mathrm{s}$. 
A graphical illustration of corresponding deformation shapes is shown in a time sequential order in Fig. 7.2. The contour plot shows the von Mises stress. The decay in the front end reaction force within $100 \mu$ s can be accounted for by the local buckling of the face ligament closest to the rigid plate as shown in the deformation at $t=70 \mu \mathrm{s}$. On the other hand, the rise and decay of the back end reaction force are inferred from the arrival of an elastic stress wave followed by face wrinkling of the face ligament at the back end, which is confirmed by the contour at $t=140 \mu$ s and the magnified view of the deformation near the back end at $\mathrm{t}=420 \mu \mathrm{s}$. The increase in the front end reaction force around $\mathrm{t}=400 \sim 600 \mu \mathrm{s}$ is explained by the densification of a cell, as shown in the zoom-in view of deformation shape near the front end at $\mathrm{t}=420 \mu \mathrm{s}$.

The high-velocity dynamic loading plots happen during the propagation of one elastic wave propagation along the column length, and the dynamic response curves can be characterized through the following parametric analysis.

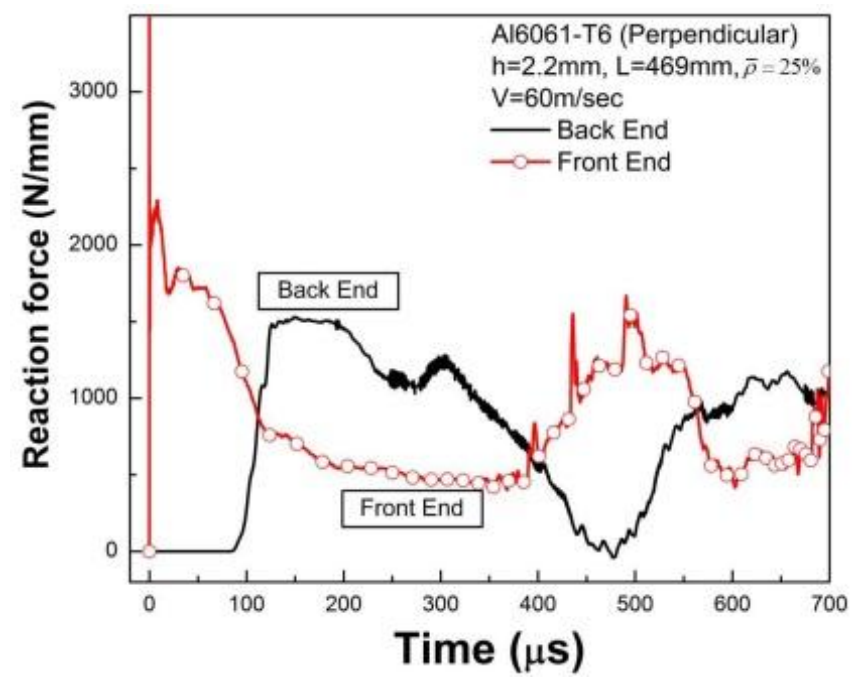

Figure 7.1: Typical reaction curve of corrugated core sandwich columns compressed perpendicular-tocorrugations. The curve shows an Al6061-T6 sandwich column of $\mathrm{h}=2.2 \mathrm{~mm}, \mathrm{~L}=469 \mathrm{~mm}, \bar{\rho}=25 \%$ $\left(\mathrm{t}=3.175 \mathrm{~mm}, \mathrm{l}=22 \mathrm{~mm}, \mathrm{w}=60^{\circ}\right)$ under $\mathrm{V}=60 \mathrm{~m} / \mathrm{s}$. 


\subsubsection{Influence on the Reaction Force Characteristics}

As demonstrated in Section 7.3.1, the high velocity response of corrugated core sandwich columns loaded perpendicular-to-corrugations is dominated by highly localized buckling deformation. In this section, in-depth analysis on the effects of applied velocity, overall column geometry, and imperfections are conducted to gain insight on the absorbed and transmitted impulse to external loading through this structure.

For applied compression velocities of $20,60,100 \mathrm{~m} / \mathrm{s}$, the reaction forces at the back and front ends are examined in Fig. 7.3 and Fig. 7.4, respectively. As shown in Fig. 7.3, all of the back end reaction forces rise after a time period of $100 \mu$ s and maintain

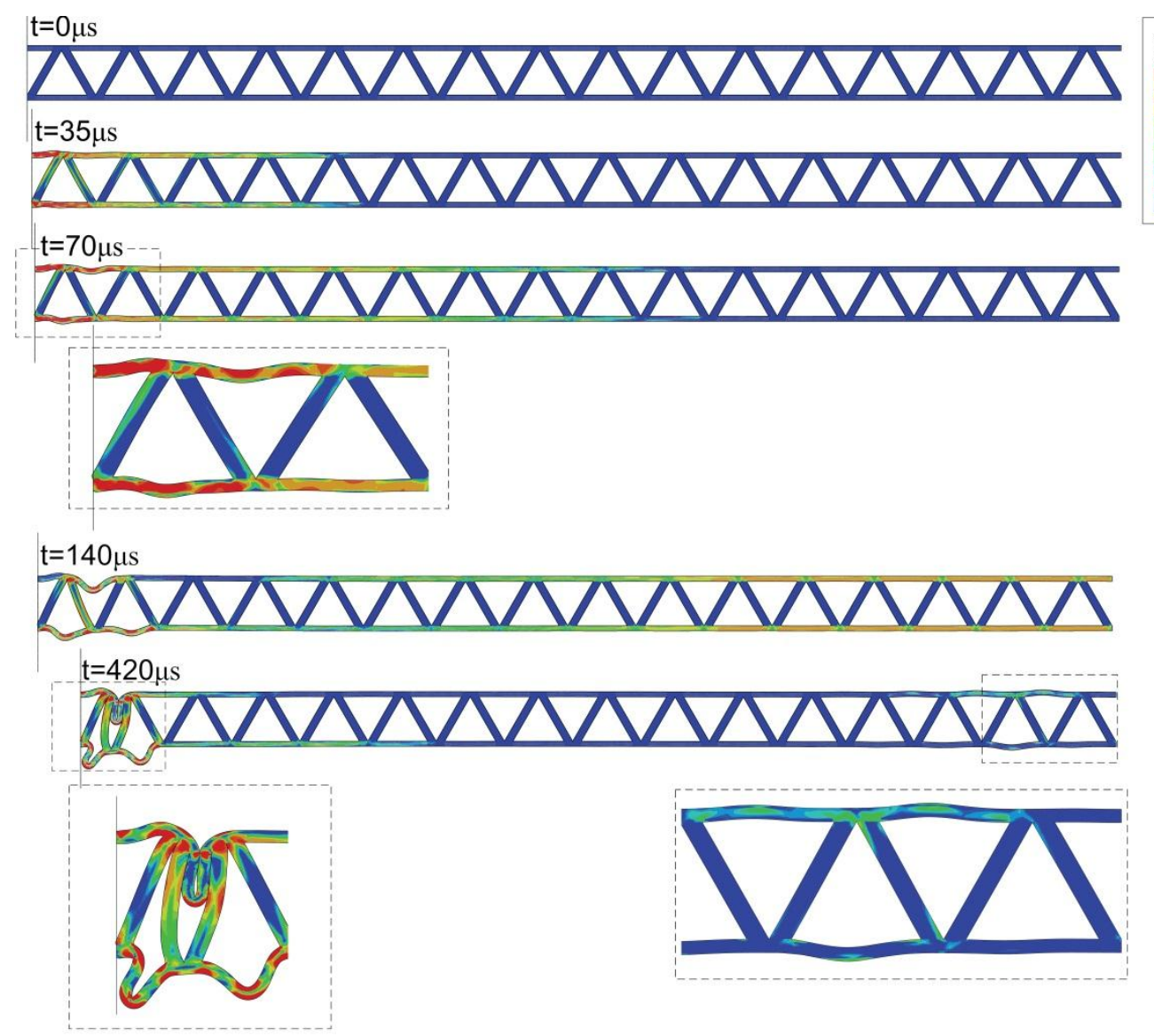

Figure 7.2: Deformation of the Al6061-T6 corrugated core sandwich columns of $\mathrm{h}=2.2 \mathrm{~mm}, \mathrm{~L}=469 \mathrm{~mm}, \bar{\rho}=$ $25 \%\left(\mathrm{t}=3.175 \mathrm{~mm}, \mathrm{l}=22 \mathrm{~mm}, \mathrm{w}=60^{\circ}\right)$ compressed perpendicular-to-corrugations under $\mathrm{V}=60 \mathrm{~m} / \mathrm{s}$. 


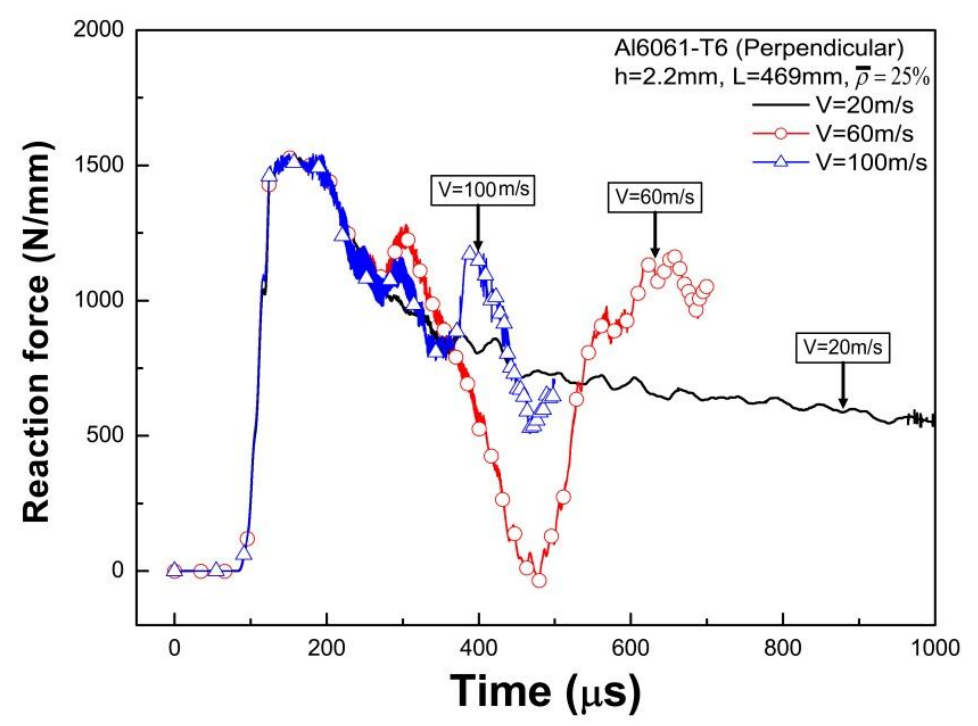

Figure 7.3: Back end reaction forces depending on applied velocities of $V=20,60,100 \mathrm{~m} / \mathrm{s}$.

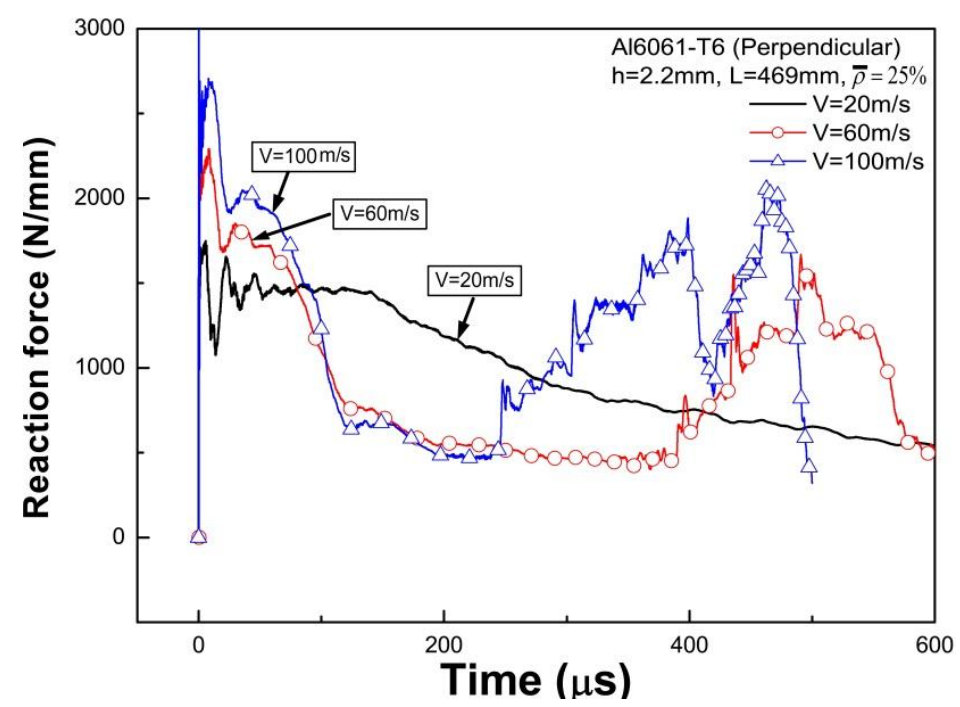

Figure 7.4: Front end reaction forces depending on applied velocities of $V=20,60,100 \mathrm{~m} / \mathrm{s}$.

their peaks at $\mathrm{P}_{\text {back }}=1500 \mathrm{~N} / \mathrm{mm}$, invariable to the applied compression velocities. In other words, the transmitted pulse to the back end is independent of loading intensities. This can be interpreted as follows: an elastic stress wave front, whose magnitude and propagation speed are independent of applied velocities, arrives and is reflected at the 


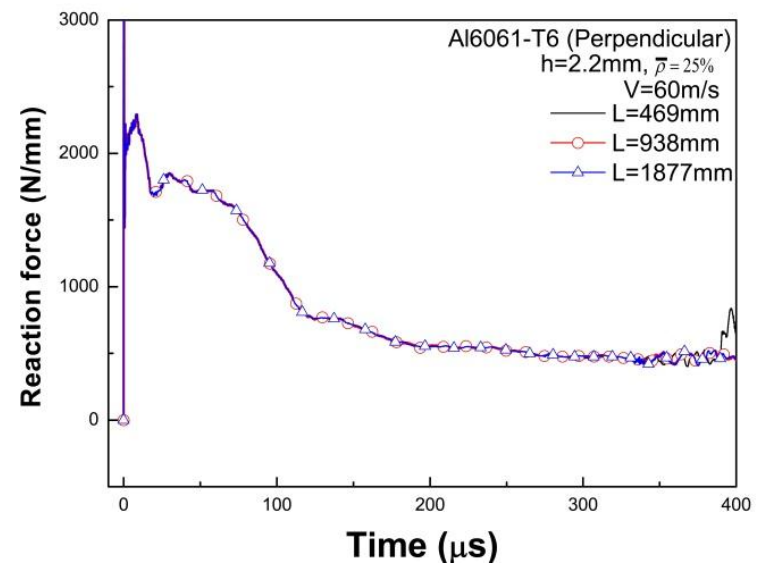

(a)

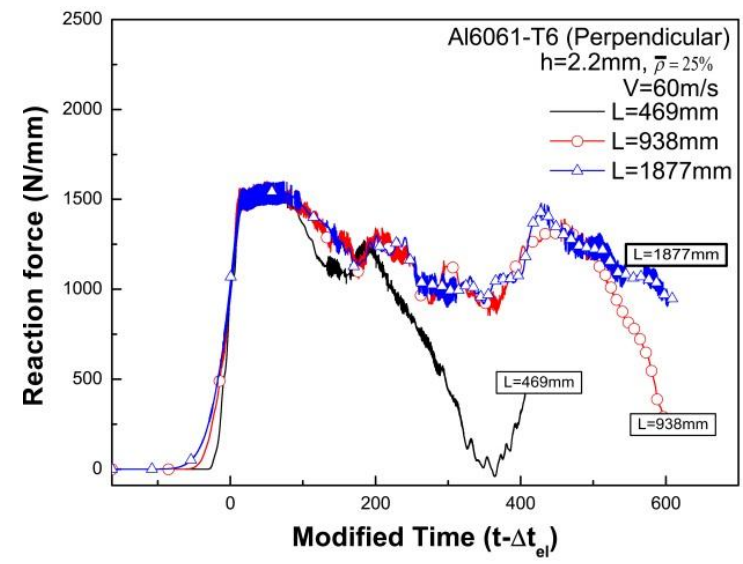

(b)

Figure 7.5: Length dependence: (a) Front end reaction force; (b) Back end reaction force.

back end. Subsequently, a face ligament near the back end buckles by the plastic wave created from the reflection of the elastic wave.

In contrast to the back end reaction forces, the front end reaction forces are dependent on the applied velocities as shown in Fig. 7.4. They drop quickly within approximately 100 microseconds. However, the increase in the front end reaction forces for $\mathrm{V}=60 \mathrm{~m} / \mathrm{s}$ and $100 \mathrm{~m} / \mathrm{s}$ are observed again at 420 and $220 \mu$ s, respectively, which result from cell densification that causes contact between face and core ligaments.

The effect of column length on the dynamic response is investigated in Fig. 7.5. Through this investigation, the response (absorbed and trasmitted pulse through this structure) accouted for by wave propagation will be corroborated. The column lengths of sandwich columns are varied as $\mathrm{L}=469,938,1877 \mathrm{~mm}$ while the same face sheet thickness and core dimensions retained. The column lengths are the same, twice and four times as long as that of that representative column as shown in subsection 7.3.1, respectively. The longer columns are expected to fail in global buckling under quasi- 
static loading. The front end reaction forces are invariable to the column lengths, thus confirming highly localized buckling.

On the other hand, each back end reaction force curve is translated along the time axis by $\Delta t_{e l}^{(\text {Perp })}$ such that the arrival time of elastic stress wave to the back end is set to zero. The pulse shape of the back end reaction curves until the load drops are insensitive to column length. Therefore, the high velocity responses are dominated by highly localized buckling deformation irrespective of column lengths. However, the longer columns ( $\mathrm{L}=938,1877 \mathrm{~mm}$ ) show slightly longer, but with negligible difference, duration at peak load than the shorter column $(\mathrm{L}=469 \mathrm{~mm})$ does.

The effects of imperfections on the dynamic response, specifically the choice of imperfection modes and their magnitudes, are investigated in Figs. 7.6 and 7.7. First, the dynamic response of an Al6061-T6 sandwich column having a global curvature is

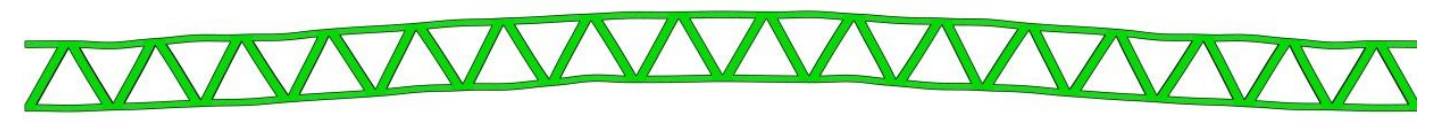

(a)

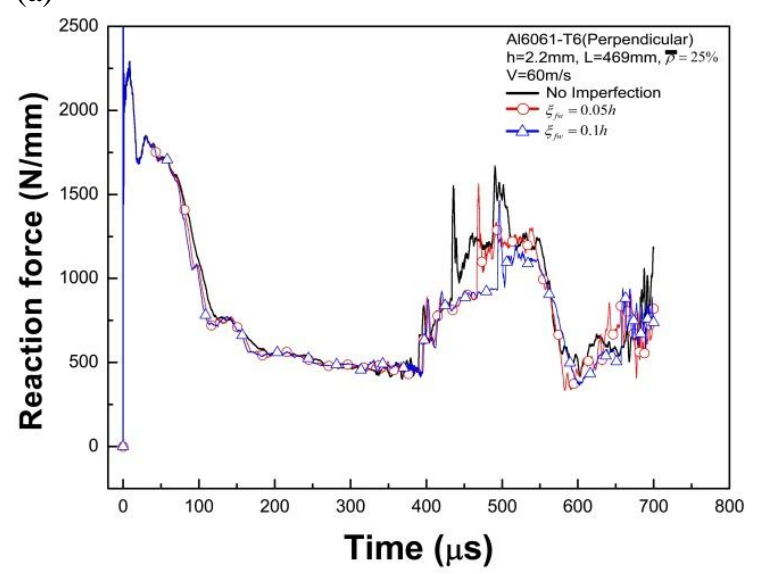

(b)

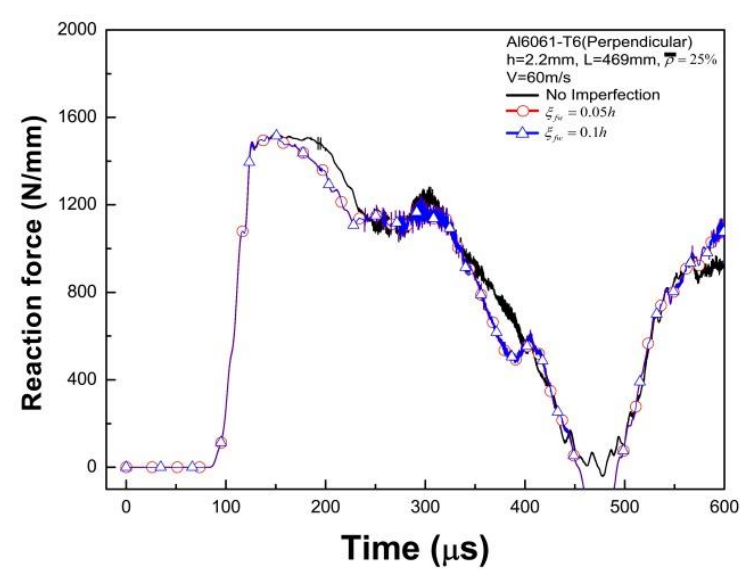

(c)

Figure 7.6: Global buckling mode (mode 7) imperfection sensitivity: (a) imposed eigenmode for the imperfection; (b) front end reaction force; (c) back end reaction force. 
investigated. To perform the dynamic FEA, a globally deformed eigenmode from static buckling analysis, (here, mode \#7 as shown in Fig. 7.6(a)) is superposed on the mesh for a perfect geometry. (Mode \#7 is the lowest globally-buckled mode whereas mode \#1 through \#20 except for the mode \#7 correspond to face-wrinkled modes.) Figs. 7.6(b) and(c) show the reaction forces at the front and back ends by varying the imperfection magnitude between $0<\xi_{\mathrm{g}}<0.1 \mathrm{c}$, respectively. However, it is demonstrated that no significant difference is found between them.

Secondly, FEA is performed on a sandwich column having a geometrically imperfect face ligaments. To represent such a geometrically imperfect column, an eigen mode of Mode \#6 from an additional static buckling analysis is incorporated into the dynamic FE simulations. (Mode \#6 is one of face-wrinkled modes among the twenty static buckling modes obtained from the static buckling analysis. And, the maximum

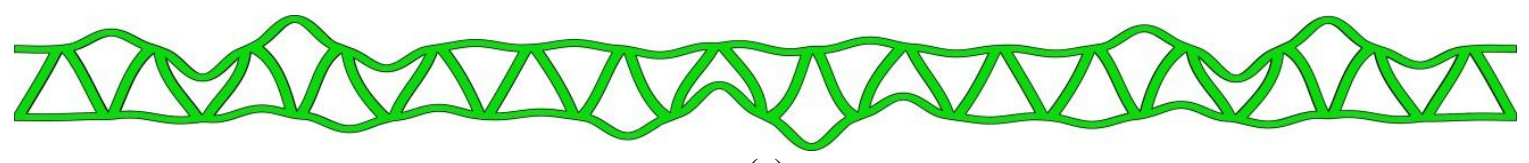

(a)

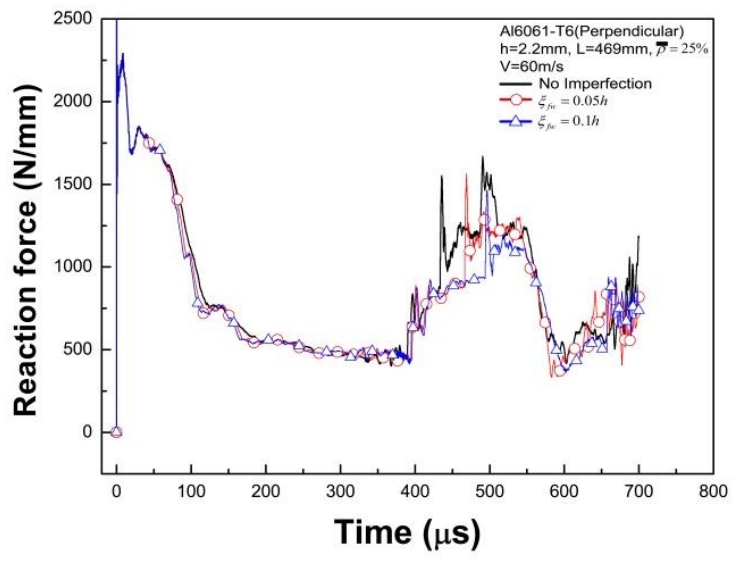

(b)

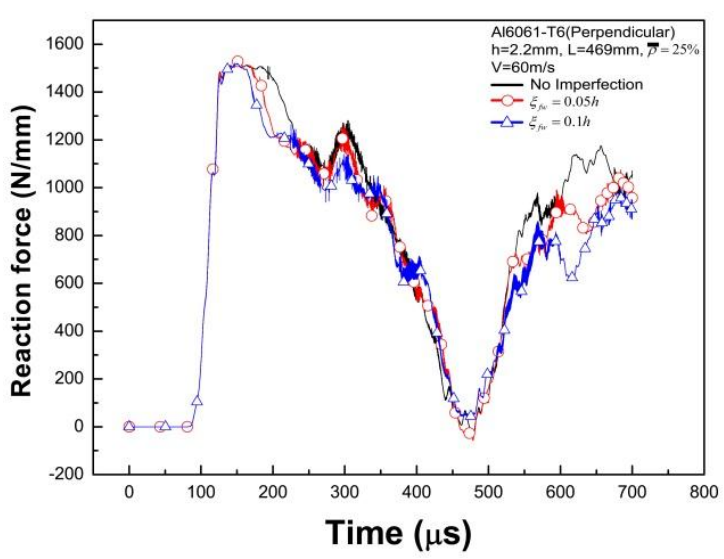

(c)

Figure 7.7: Face wrinkling mode (mode 6) imperfection sensitivity: (a) front end reaction force; (b) back end reaction force. 
wrinkled faces in the selected mode are located in both back and front ends.) Although this type of imperfection is identical to the deformation characteristics of sandwich columns under high velocity compression perpendicular-to-corrugations, there is no significant difference in the front end reaction forces at the imperfection range of $0<\xi^{(\mathrm{f})}<$ 0.1h as shown in Fig. 7.7(a). It is noted that the imperfection only affects a pulse duration of the back end reaction force at $\mathrm{P}=1500 \mathrm{~N} / \mathrm{mm}$ slightly as shown in Fig. 7.7(b).

To summarize the observations in Figs. 7.6 and 7.7, the considered range of imperfections does not cause appreciable differences to the front end reaction force curves due to intense local buckling deformation accompanying substantial plastic deformation at the front end. However, the pulse duration in the back end reaction force is slightly affected by the imperfection in the considered imperfection range. Because a mild intensity of back end reaction force, corresponding to yield stress, is maintained until the face ligament buckles, the duration will be affected by the imperfections: Put in another way, the face wrinkling near the back end is analogous to the dynamic buckling problem of a plastic column under a constant load associated with $\sigma_{\mathrm{Y}}$.

\subsubsection{Analytic Simplification of Reaction Forces}

From the findings in the previous sections, reaction forces of corrugated core sandwich columns compressed perpendicular-to-corrugations can be modeled as shown in Fig. 7.8. The front end reaction force is characterized in an initial rise and drop followed by repeated pulses due to cell densifications causing contact between face and core members. The back end reaction force rises after the elastic stress wave arrives at the back end, maintains its peak for a period, and then drops due to excessive deformation of 


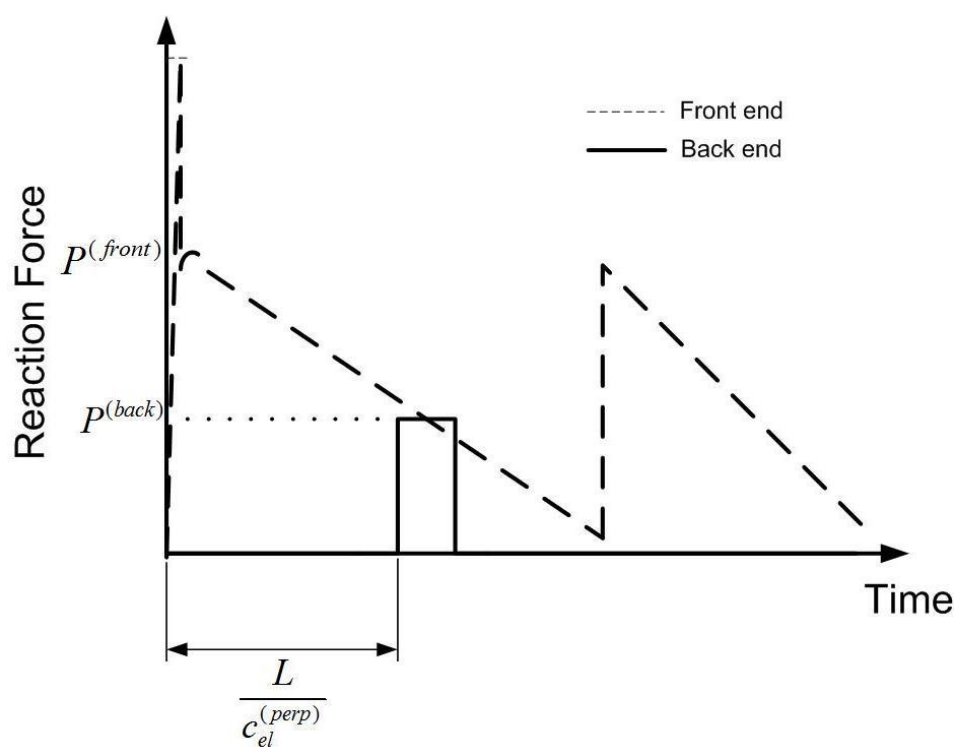

Figure 7.8: Simplified model of the front and back end reaction force curves for corrugated core sandwich columns under high-velocity compression perpendicular-to-corrugations.

a face ligament near the end. Thus, the peak duration is related to the slenderness ratio of a face ligament as long as there is no interfering pulse (e.g., contact forces near the front end).

Correspondingly, the peak loads can be deduced from the theory of rateindependent elastic-plastic wave propagation using an analogy between sandwich columns and monolithic columns. The peak of front end reaction force curve is given by $[3-5,13]$

$$
\frac{P^{(\text {front })}}{P_{Y}}=1+\left(\sqrt{\frac{E_{T}^{\prime}}{E /\left(1-v^{2}\right)}}\right)\left(\frac{V}{c_{e l}^{(\text {perp })} \cdot \varepsilon_{Y}^{\prime}}-1\right)
$$

where $c_{e l}^{(\text {Perp })}, E_{T}^{\prime}, \epsilon_{Y}^{\prime}$ denote the apparent elastic wave speed, tangent modulus in plane strain condition, yield strain in plane strain condition, $\epsilon_{Y}^{\prime} \approx\left(\frac{\sigma_{Y}}{\sqrt{1-v_{P}+v_{P}^{2}}}\right) /\left(\frac{E}{1-v^{2}}\right)$, and 
$\mathrm{P}_{\mathrm{Y}}$ is the in-plane load associated with yield stress, $\mathrm{P}_{Y}=\frac{2 h \sigma_{Y}}{\sqrt{1-v_{P}+v_{P}^{2}}}$.

On the other hand, the upper and lower bounds of back end reaction force can be obtained assuming the buckling of a face ligament near the back end either by a propagating elastic wave or by its reflected wave.

$$
\begin{gathered}
\frac{P_{\text {lower }}^{(\text {back })}}{P_{Y}} \approx 1 \\
\frac{P_{\text {upper }}^{\text {(back })}}{P_{Y}} \approx 1+\sqrt{\frac{E_{T}^{\prime}}{E /\left(1-v^{2}\right)}}
\end{gathered}
$$

In Fig. 7.9, the analytic expressions given in Eqns. (7.1) and (7.2) are compared with FE results of sandwich columns having several face sheet thicknesses of $h=1.31,2.2$, and $4.4 \mathrm{~mm}$ subjected to various applied velocities. Eqn. (7.1) for $P^{(\text {front })}$ and Eqn. (7.2) for $P_{\text {lower }}^{(\text {back }}$ and $P_{\text {upper }}^{(\text {back })}$, are plotted using red dash lines and black straight lines, respectively. The peaks in back end reaction force computed from FEM are bounded by analytical expressions of Eqn. (7.2) while the analytical expression for the peaks in front end reaction force, $P^{(\text {front })}$, Eqn. (7.1), underpredicts the FE front end peaks.

The under-prediction can be attributed to neglecting the core contribution to inplane stiffness in the analytic expression of Eqn. (7.1). As seen in Fig. 7.10(a), the major core deformation mechanism by a low-velocity compression is folding at nodes between face sheets and a core so that the contribution of a core to the in-plane stiffness of sandwich columns is minimal. This has been already confirmed through verification of apparent stress wave speeds, $c_{e l}^{(S W)}$ in Chapter 3, where the analytic expression for the wave propagation speeds is based on neglecting core's in-plane stiffness. 
However, the deformation of a core strut positioned closest to the front end under high velocity compression differs from the deformation mechanism of the other core struts or the deformation mechanism observed under a low velocity compression. The deformation of the core strut closest to the front end is instantaneous so that considerable axial compression and flexural deformation is involved. An exaggerated illustration for the description of core deformation is sketched in Fig. 7.10(b). In this regard, Eqn. (7.1) is modified into Eqn. (7.3) by multiplying a correction factor to take the core contribution into account. The correction factor, $\mathrm{f}_{\text {correction }}$, is the ratio of the in-plane stiffness of a sandwich column with and without the core's in-plane contribution. The derivation of $\mathrm{E}_{\text {core }}^{*}$ in Eqn. (7.3), an effective in-plane core stiffness explaining the core deformation mechanism, is included in Appendix E.

$$
\begin{aligned}
&\left.\frac{P^{(\text {front })}}{P_{Y}}\right|_{\text {modified }}=f_{\text {correction }} \cdot\left[1+\left(\sqrt{\frac{E_{T}^{\prime}}{E /\left(1-v^{2}\right)}}\right)\left(\frac{V}{c_{\text {el }}^{(\text {perp })} \cdot \varepsilon_{Y}^{\prime}}-1\right)\right] \\
& \text { where } \quad f_{\text {correction }}=\left(\frac{2 h E /\left(1-v^{2}\right)+c E_{\text {core }}^{*}}{2 h E /\left(1-v^{2}\right)}\right) \\
& E_{\text {core }}^{*}=\frac{(\cos \omega)^{3}}{\sin \omega} \frac{t E}{l\left(1-v^{2}\right)}
\end{aligned}
$$

The modified equation, Eqn. (7.3), is also plotted in Fig. 7.9 and improves the $P^{\text {(front })}$ prediction. 


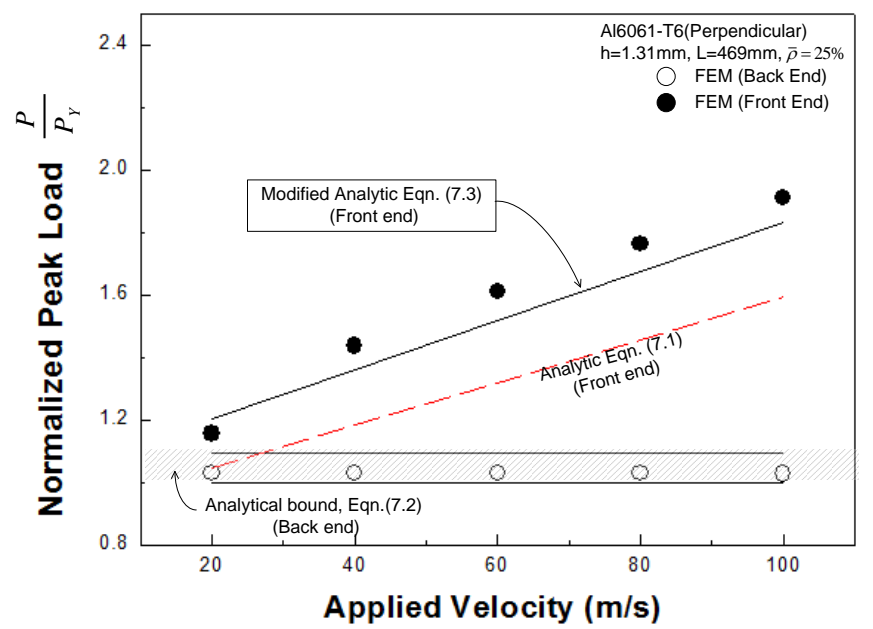

(a)

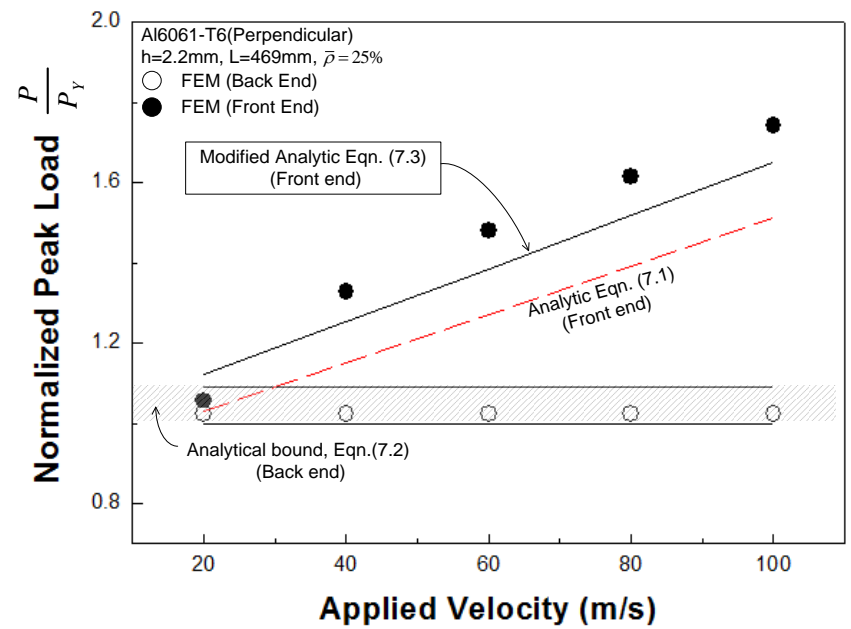

(b)

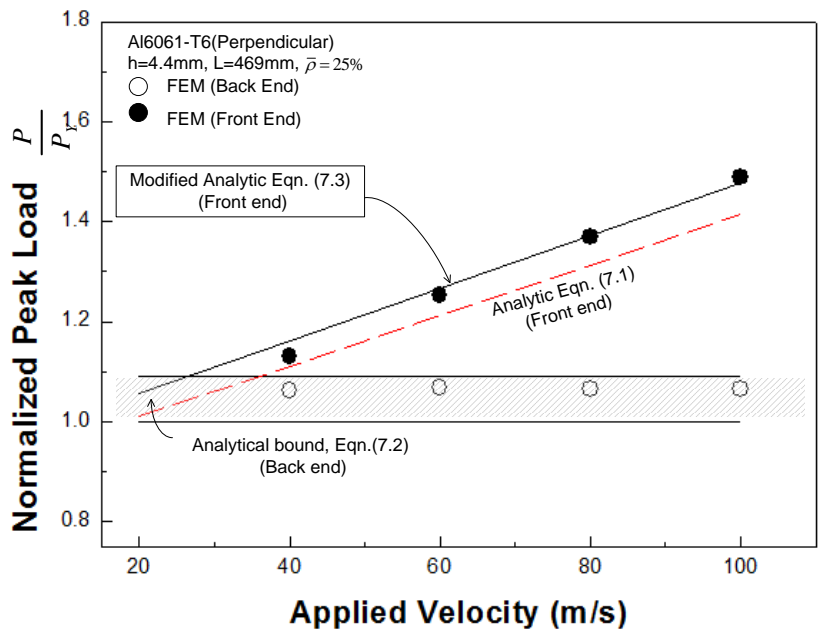

(c)

Figure 7.9: Analytic prediction of peak reaction forces of corrugated core sandwich columns under high velocity compression perpendicular-to-corrugations: (a) $h=1.31 \mathrm{~mm}$; (b) $h=2.2 \mathrm{~mm}$; (c) $h=4.4 \mathrm{~mm}$. 


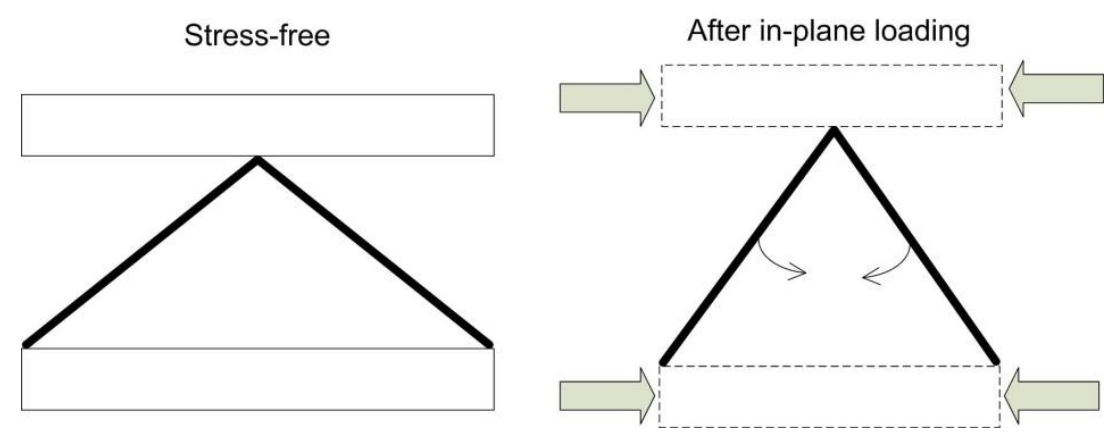

(a) low velocity

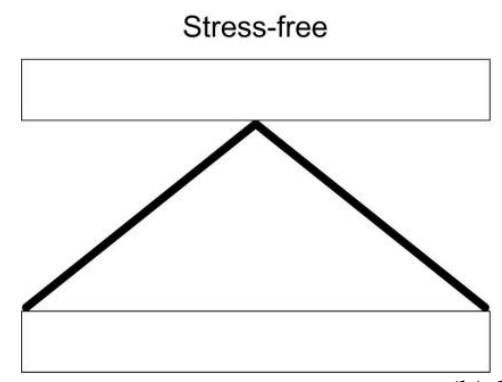

(b)

(b) high velocity

Figure 7.10 : Core contribution to in-plane stiffness depending on the magnitude of compression velocity: (a) low velocity; (b) high velocity.

\subsubsection{Material Rate Dependence}

So far in this section, the FE observations and analytic characterization have been focused on corrugated core sandwich columns fabricated from rate independent materials (A16061-T6). In this subsection, how the material strain rate dependence affects the high velocity dynamic response is discussed through FE analysis of SS304 corrugated core sandwich columns.

FE simulations are carried out for SS304 corrugated core sandwich columns of $\mathrm{h}=2.5 \mathrm{~mm}, \mathrm{~L}=707 \mathrm{~mm}$ subject to $\mathrm{V}=40 \mathrm{~m} / \mathrm{s}$ with and without considering material strainrate dependence. The FE simulation without material strain-rate dependence is computed by suppressing the dynamic strengthening enhancement ratio, $R(\dot{\mathrm{p}})$, in the parent material description. The reaction forces from the two FE computations are compared in Fig. 7.11. It is evident that the higher magnitude in the rate-dependent simulation 
attributes to the elevation in dynamic yield strength, i.e. the effects of material strain-rate dependence.

To examine whether the rate-dependent result can be inferred from the rateindependent computation, the rate-independent back and front end reaction curves are scaled with $R(\dot{p})$, and then the scaled curves are compared to those of the rate-dependent simulation in Fig. 7.12. As a result, the reaction force curves from the rate-dependent FE simulation and the R-scaled curves from the rate-independent simulations, which are created by multiplying the curves by $\mathrm{R}(\dot{\mathrm{p}})$, show similarity in magnitude and shape.

In the high velocity region, most of deformation is plastic so that the rate-dependent reaction force curves of corrugated core sandwich columns compressed perpendicular-tocorrugations can be approximated by multiplying $\mathrm{R}(\dot{\mathrm{p}})$, a function of plastic strain rate, from the rate independent simulation.

Accordingly, the peak forces considering the material strain-rate dependence of a parent material whose plastic part is modeled by two plastic hardening moduli such as SS304 can be obtained by simply multiplying the rate-independent expressions by $R(\dot{\mathrm{p}})$.

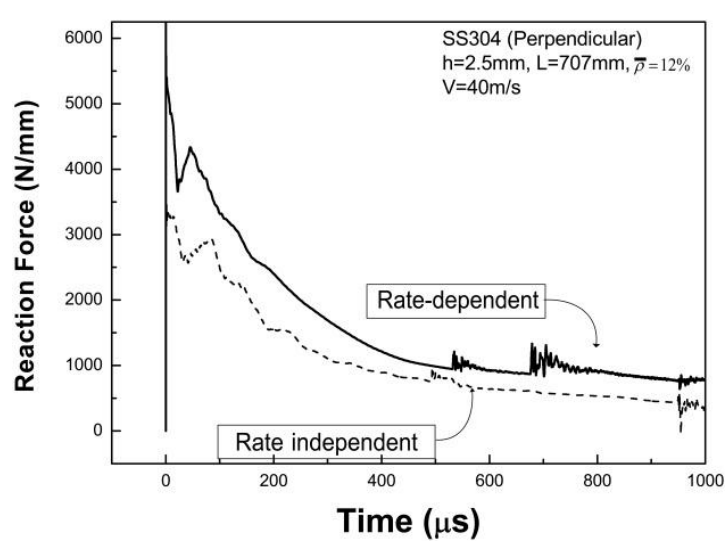

(a)

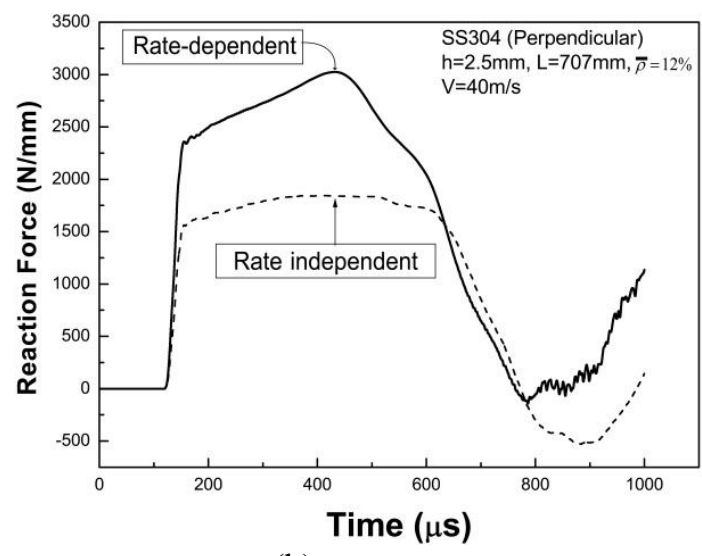

(b)

Figure 7.11: The comparison of reaction forces between rate-dependent FEA and rate-independent FEA: (a) front end reaction force; (b) back end reaction force. 


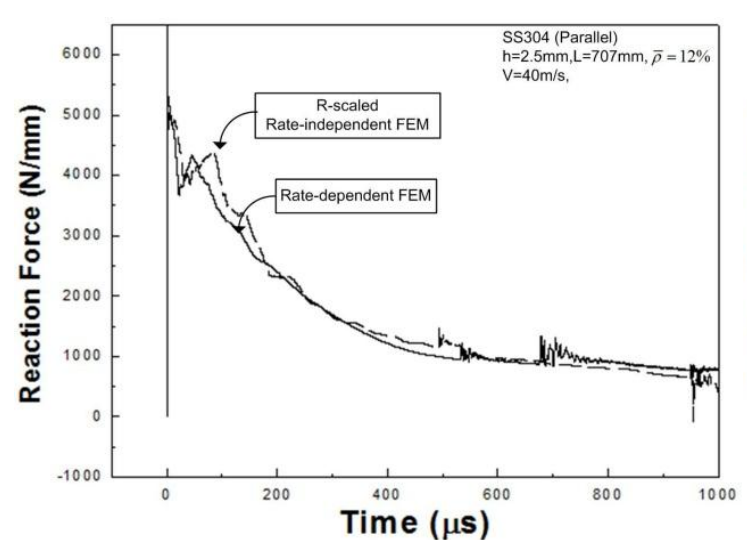

(a)

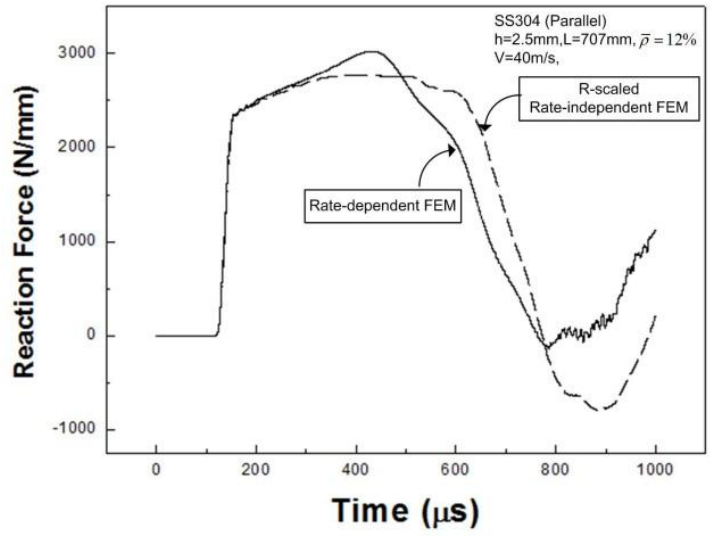

(b)

Figure 7.12 : Comparison between R-scaled rate-independent analysis and the rate-dependent analysis, (a) front end reaction force, (b) back end reaction force.

$$
\begin{gathered}
\frac{P^{(f r o n t)}}{P_{Y}^{(0)}} \mid \approx R(\dot{p}) \cdot f_{\text {correction }} \cdot\left[\frac{P_{Y}^{\prime(1)}}{P_{Y}^{\prime(0)}}+\sqrt{\frac{E_{T}^{\prime(2)}}{E /\left(1-v^{2}\right)}}\left(\frac{V}{c_{e l}^{(0)(\text { perp })} \varepsilon_{Y}^{\prime(0)}}-1\right)-\sqrt{\frac{E_{T}^{\prime(2)}}{E_{T}^{\prime(1)}}} \frac{\left(P_{Y}^{\prime(1)}-P_{Y}^{(0)}\right)}{P_{Y}^{\prime(0)}}\right] \\
\frac{P_{\text {upper }}^{(\text {back })}}{P_{Y}^{\prime(0)}} \approx R(\dot{p}) \cdot\left[\frac{P_{Y}^{\prime(1)}}{P_{Y}^{\prime(0)}}+\sqrt{\frac{E_{T}^{\prime(2)}}{E /\left(1-v^{2}\right)}}-\sqrt{\frac{E_{T}^{\prime(2)}}{E_{T}^{\prime(1)}}} \cdot \frac{P_{Y}^{\prime(1)}-P_{Y}^{\prime(0)}}{P_{Y}^{\prime(0)}}\right] \\
\frac{P_{\text {lower }}^{(\text {back })}}{P_{Y}^{\prime(0)}} \approx R(\dot{p})
\end{gathered}
$$

In the above equations, the third term in the RHS of Eqn. (7.5) and the second term in the RHS of Eqn. (7.6) result from the bilinear modeling of the plastic part in SS304. The superscripts (1) and (2) represent the respective plastic regions while (0) represents the elastic region, as denoted in the $\sigma_{11}-\varepsilon_{11}$ diagram of Fig. 7.13. Although the relationship between the axial stress versus and the axial strain in the presence of constraint in lateral expansion (i.e., the plane-strain condition) does not generally show a strict discontinuity point around the region of the elastic-plastic transition [97], the relationship between $\sigma_{11}$ and $\varepsilon_{11}$ is assumed as shown in Fig. 7.13, for convenience. 


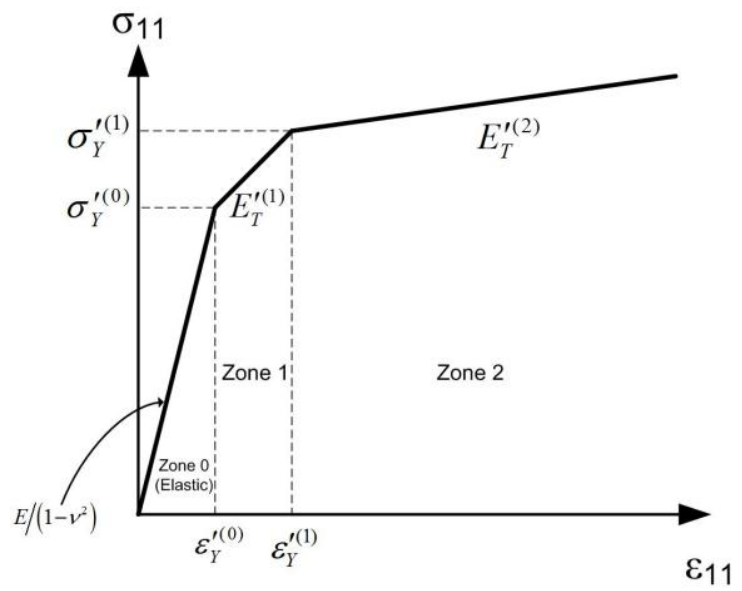

Figure 7.13: Approximated $\sigma_{11}-\varepsilon_{11}$ diagram of an elastic-bilinear plastic material (SS304) in the presence of lateral expansion constraint.

Hence, $\mathrm{E}_{T}^{\prime(k)}$ and $\mathrm{P}_{Y}^{\prime(k)}$ are computed from Eqn. (2.35) and from the relation of $\mathrm{P}_{Y}^{\prime(k)}=$ $2 h \cdot \sigma_{Y}^{\prime(k)} \approx 2 h \cdot \frac{\sigma_{Y}^{(k)}}{\sqrt{1-v_{P}+v_{P}^{2}}}$, respectively.

In Fig. 7.14, the analytical equations (7.5) through (7.7) are plotted with the FE calculations for the high velocity response of SS304 corrugated core sandwich columns having $h=1.25$ and $2.5 \mathrm{~mm}$ with the same $\mathrm{L}$ and $\bar{\rho}$ as the column presented in Figs. 7.11 and 7.12.

To sum up, the high velocity response of corrugated core sandwich columns compressed perpendicular-to-corrugations is sensitive to face wrinkling, in which a face ligament near the front end fails while an incident plastic wave passes one unit cell, and the cell near the back end buckles due to the plastic wave generated by the reflection of an incident elastic stress wave. This local-buckling dominant deformation can be also confirmed by the response comparison of sandwich columns with varied column lengths. The front and back reaction force curves can be analytically characterized by utilizing the theory of rate-independent stress wave propagation. 


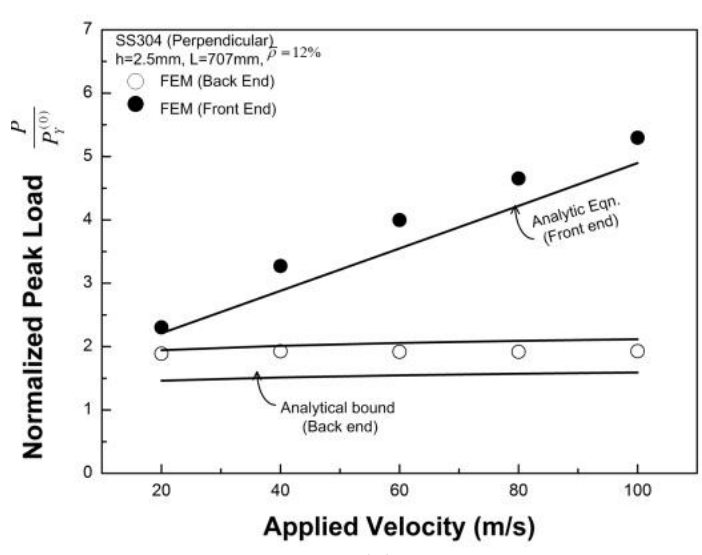

(a)

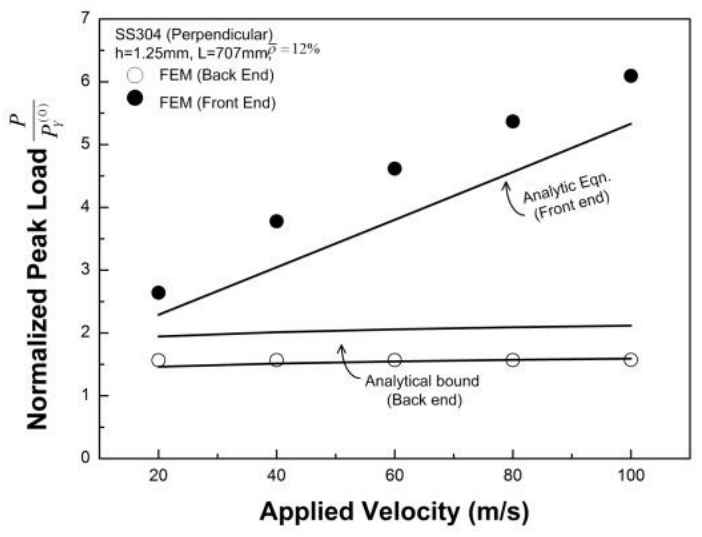

(b)

Figure 7.14: Analytic prediction of peak reaction forces of rate-dependent (SS304) corrugated core sandwich columns under high velocity compression perpendicular-to-corrugations: (a) h=1.25mm; (b) h=2.5mm.

From the comparative observations of SS304 corrugated core sandwich columns with and without consideration of the material strain-rate dependence, it is confirmed that the response of a rate-dependent corrugated core sandwich column is successfully approximated by the R-scaled response from the rate-independent analysis.

\subsection{HIGH VELOCITY RESPONSE OF SANDWICH COLUMNS (PARALLEL-TO-}

\section{CORRUGATIONS)}

The high velocity response of corrugated core sandwich columns compressed parallel-to-corrugations is investigated by utilizing FEA, followed by an analytical characterization. The initial investigation focuses on the response of the rate-independent (A16061-T6) sandwich columns of varied geometric dimensions subject to compression velocities up to $100 \mathrm{~m} / \mathrm{s}$ with material rate effects excluded. Then, simple analytic expressions are suggested in order to incorporate general features in the observed 
response characteristics of the rate-independent sandwich column responses into the model.

To account for the influence of material rate-dependence, FE analysis on a ratedependent (SS304) sandwich column is performed. Through comparing the results with and without the rate-dependence of the parent material description, a method of incorporating the material effect into the analytic expressions is suggested.

\subsubsection{General Observation}

Representative reaction forces numerically measured at the front and back ends are plotted in Fig. 7.15. The demonstrated curves are on an Al6061-T6 corrugated core sandwich column compressed parallel-to-corrugations at $\mathrm{V}=60 \mathrm{~m} / \mathrm{s}$, having the same geometric dimensions of $\mathrm{h}=2.2 \mathrm{~mm}, \mathrm{~L}=469 \mathrm{~mm}, \bar{\rho}=0.25$ as that presented in Section 7.3.1.

Upon compression at the front end, the front end reaction force rises

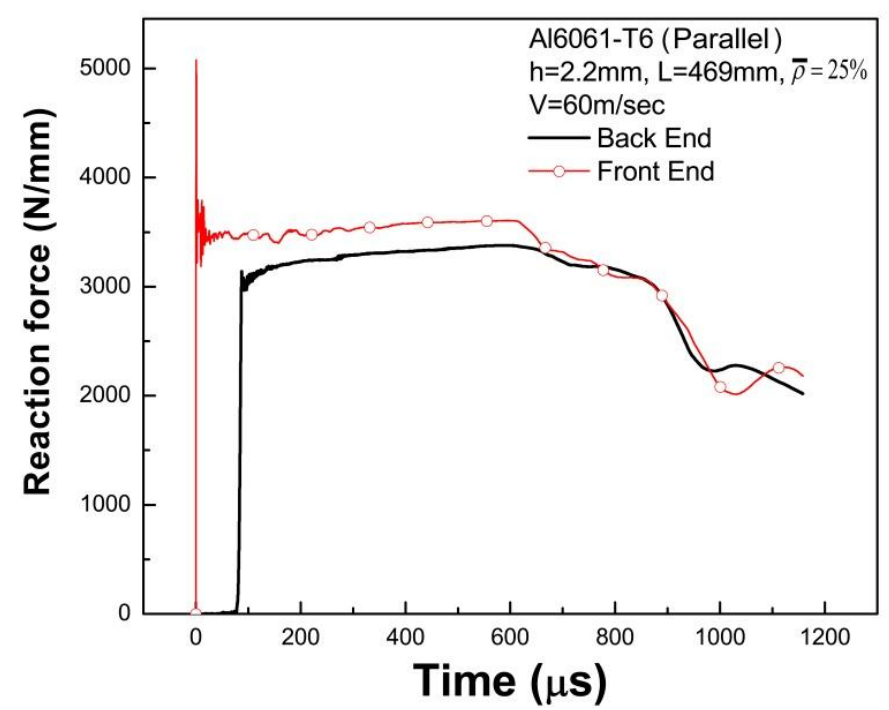

Figure 7.15: Representative reaction force curves of a corrugated core sandwich column compressed parallelto-corrugations. The curves are regarding an Al6061-T6 sandwich column of $\mathrm{h}=2.2 \mathrm{~mm}, \mathrm{~L}=469 \mathrm{~mm}, \bar{\rho}=$ $25 \%\left(\mathrm{t}=3.175 \mathrm{~mm}, \mathrm{l}=22 \mathrm{~mm}, \mathrm{w}=60^{\circ}\right)$ under $\mathrm{V}=60 \mathrm{~m} / \mathrm{s}$. 


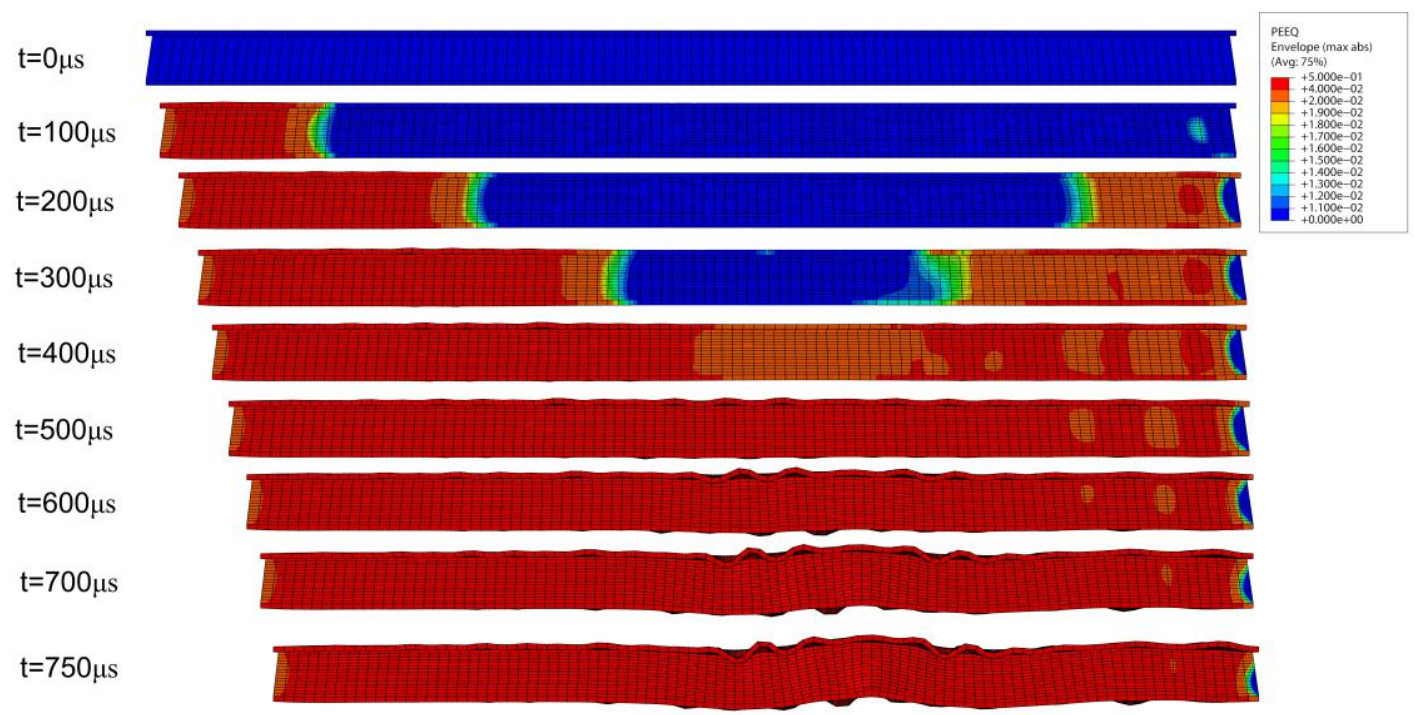

Figure 7.16: Wave propagation in the representative sandwich column presented in Fig.7.15.

instantaneously to approximately $3500 \mathrm{~N} / \mathrm{mm}$, and remains at this magnitude for several hundreds of microseconds, which differs from the front end reaction characteristics of the perpendicular-to-corrugations results in Section 7.3. On the other hand, the back end reaction force rises after a delay of $100 \mu$ s, and then, a constant reaction force is maintained for several hundreds of microseconds.

The dynamic event in the corrugated core sandwich column is described in a time sequential order in Fig. 7.16. Equivalent plastic strain is identified by the colored contour plot on the deformed column. Upon compression at the front end (left), two distinct wave fronts, plastic wave front (red) and elastic wave front (not indicated in the contour plot), appear simultaneously as shown at $\mathrm{t}=100 \mu \mathrm{s}$. At this point, the elastic wave propagation is not shown in the contour because the contour represents equivalent plastic strain, however, the elastic wave front travelling at the higher wave speed must have arrived at the back end before $t=100 \mu$ s. The elastic wave front is reflected just before $t=100 \mu$ s and transformed into a plastic stress wave proceeding toward the front end as demonstrated in 
the contours of $\mathrm{t}=200$ and $300 \mu \mathrm{s}$. The two plastic wave fronts, which are the incident and reflected plastic waves, meet at about mid-point of the column length as shown in the contours of $t=400$ and $500 \mu$ s. Subsequently, the superposition of the two plastic wave leads to local and global buckling growth, which is coincident with the drops of the reaction force curves.

Therefore, a considerable amount of information in the reaction force characteristics can be explained by elastic-plastic wave propagation.

\subsubsection{Influences on the Reaction Force Characteristics}

A parametric analysis is carried out with emphasis on the effects of compression velocity, column length, and imperfections.

The front and back end reaction forces of the sandwich column, as a function of applied compression velocities are investigated in Figs. 7.17(a) and (b), respectively. The column has the same geometric dimensions as the one presented in Figs. 7.15 and 7.16. The magnitude of the front end reaction forces increases in proportion to compression velocity from 20 to $100 \mathrm{~m} / \mathrm{s}$ while the back end reaction forces are invariant to the magnitude of compression velocity.

The investigation of the dynamic response of sandwich columns having different column lengths is illustrated in Fig. 7.18. This column length investigation will identify the wave propgation phenomenon within this structure and provide an insight on the corresponding pulse characteristics. The sandwich columns have column lengths twice $(\mathrm{L}=938 \mathrm{~mm})$ and three times $(\mathrm{L}=1407 \mathrm{~mm})$ as long as that $(\mathrm{L}=469 \mathrm{~mm})$ presented in Fig. 


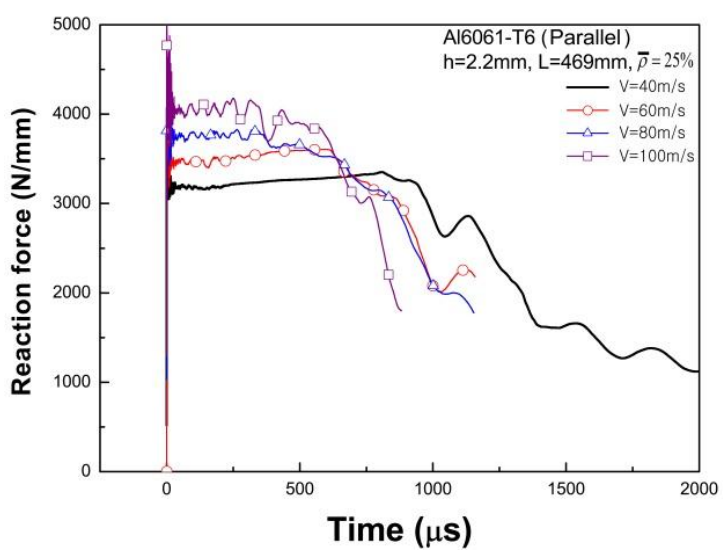

(a)

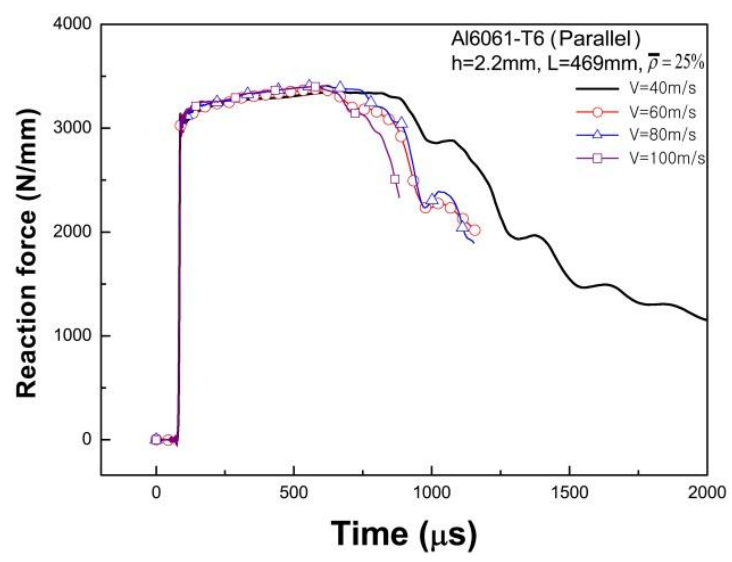

(b)

Figure 7.17: Velocity-dependent reaction forces at (a) the front end; (b) the back end.

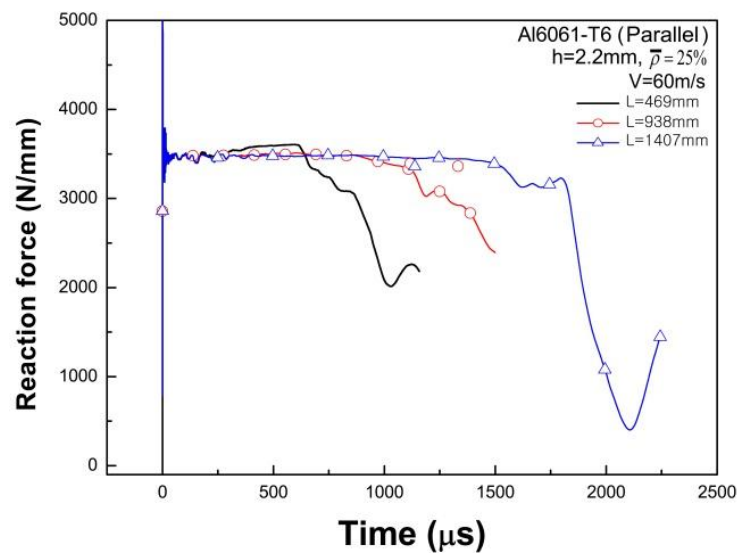

(a)

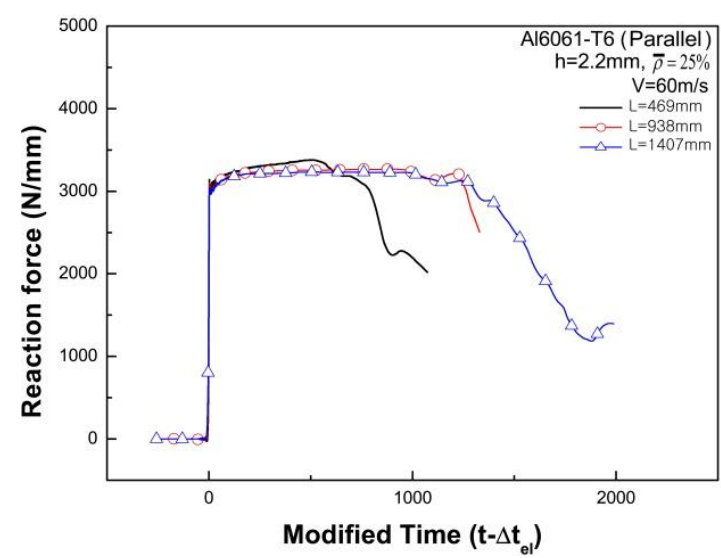

(b)

Figure 7.18: Effects of column length at V=60m/s: (a) front end reaction force; (b) back end reaction force.

7.15 , with the same face sheet thickness $(h=2.2 \mathrm{~mm})$ and core dimensions $(\bar{\rho}=0.25)$ retained.

As shown in Fig. 7.18(a), the durations of front end reaction forces are clearly dependent on the column lengths. With regard to back end reaction forces, each back end reaction force curve is translated along the time axis by $\Delta t_{e l}^{(\text {Para })}$ such that the arrival time of each elastic stress wave to the back end coincides. The transformed back end reaction curves are shown in Fig. 7.18(b) with the wave propagaion of a longer sandwich column of L=938mm in Fig. 7.19. From the illustrated contours in Figs. 7.14 and 7.19, 


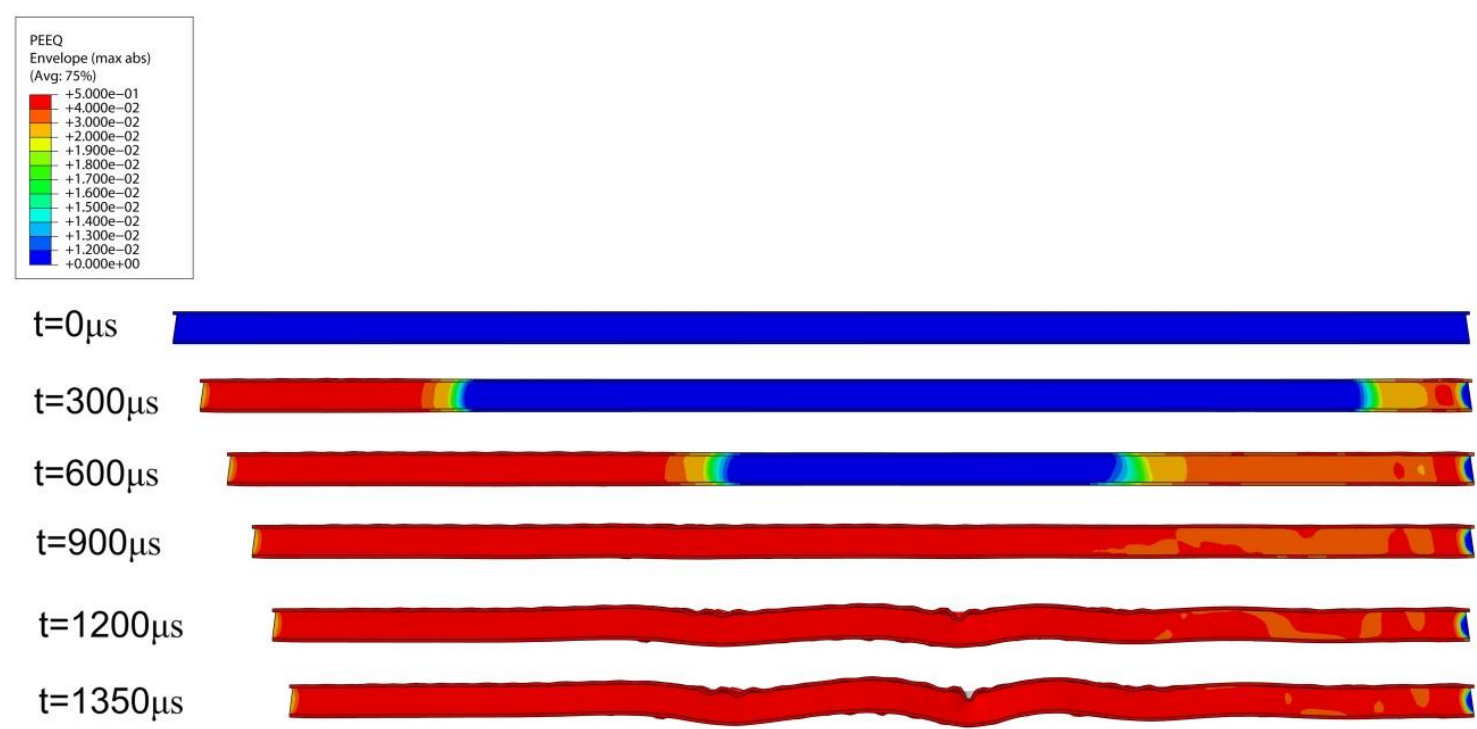

Figure 7.19. Sandwich column length dependence ( $\mathrm{L}=938 \mathrm{~mm})$.

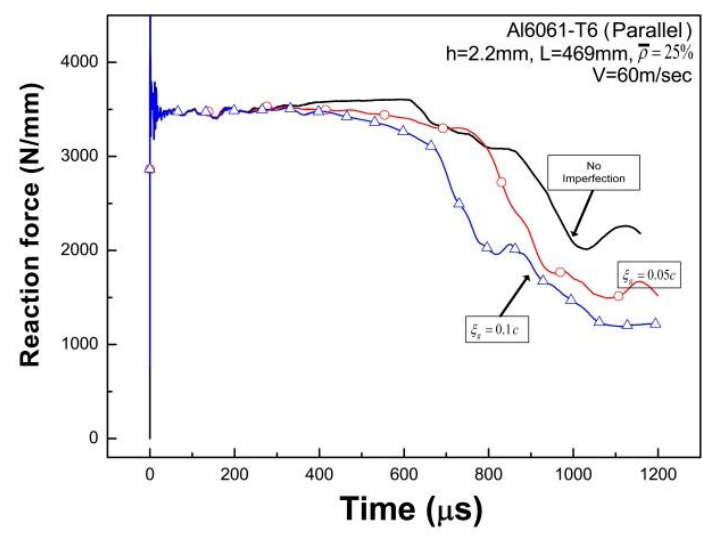

(a)

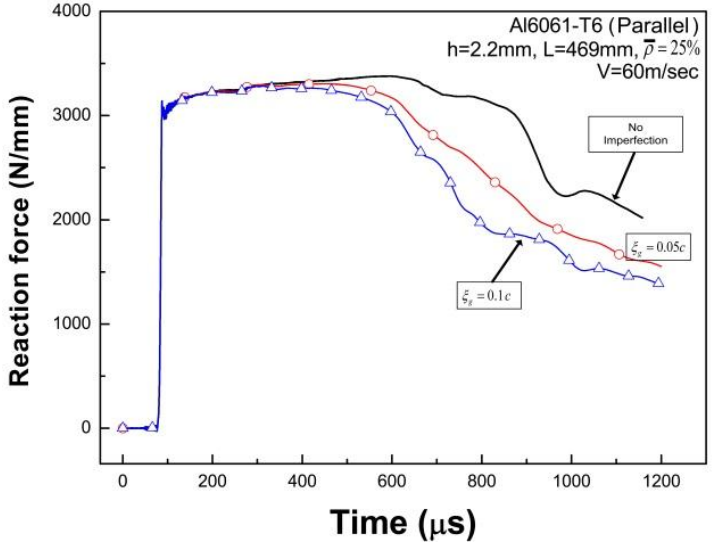

(b)

Figure 7.20: Imperfection-sensitive reaction force curves: (a) Front end reaction force; (b) Back end reaction force curves.

the excessive deformation initiated near the superposition of the two plastic waves leads to the termination of maintaining a constant level of reaction forces.

To investigate the effects of imperfections, FE analysis on an Al6061-T6 corrugated core sandwich column with a global initial curvature are performed by varying its magnitude up to $\xi=0.1 c$. The column has the same geometric dimensions as that presented in Section 7.4.1. The varied magnitudes of the assigned global curvature 


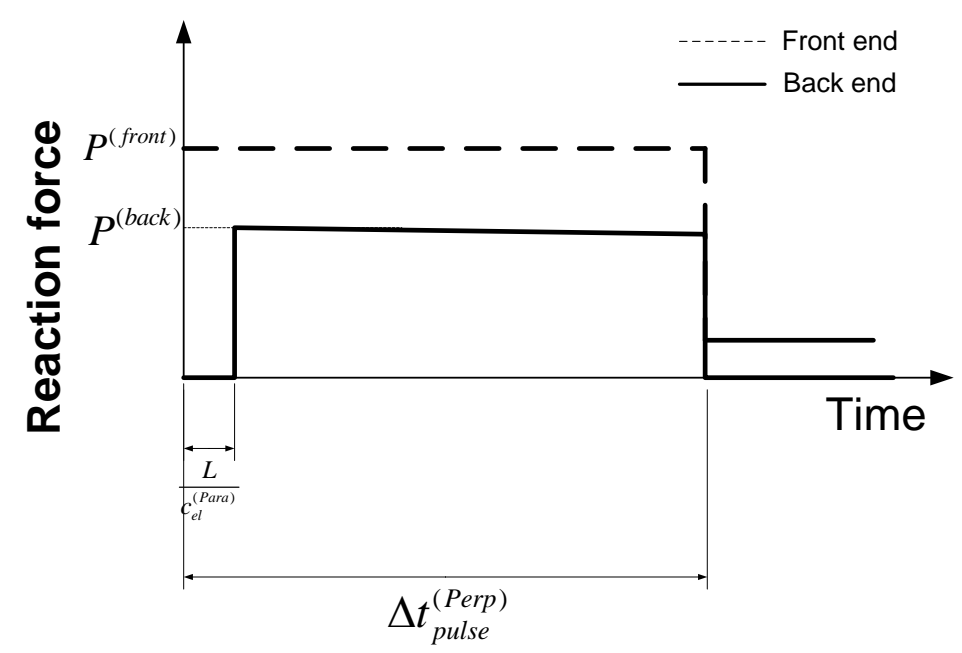

Figure 7.21: Simplification of analytical model.

influence the duration of each end reaction force with minimal impact on the constant level of reaction force curves as shown in Fig. 7.20.

\subsubsection{Analytic Simplification}

From the previous investigations, the front end reaction force reveals an initial rise followed by a constant peak maintained for some period, and similarly, back end reaction force shows similar response to the front end reaction force after a delay, which is taken for the arrival of an elastic wave, $L / c_{e l}^{(\text {Para })}$. Accordingly, the reaction force curve characteristics can be simplfied as shown in Fig. 7.21.

Much information about the reaction force characteristics can be explained by elastic-plastic wave propagation. For a rate independent material, peak reaction forces of the front and back end reaction characteristics, $\mathrm{P}^{(\text {front })}$ and $\mathrm{P}^{(\text {back })}$, can be given by

$$
\frac{P^{(\text {front })}}{P_{Y}^{(\text {para })}} \approx 1+\left(\sqrt{\frac{2 h E_{T}^{\prime}+\bar{\rho} c E_{T}}{2 h E /\left(1-v^{2}\right)+\bar{\rho} c E}}\right)\left(\frac{V}{c_{e l}^{(\text {para })} \cdot \varepsilon_{Y}^{\prime}}-1\right)
$$


and

$$
\frac{P^{(\text {back })}}{P_{Y}^{(\text {para })}} \approx 1+\sqrt{\frac{2 h E_{T}^{\prime}+\bar{\rho} c E_{T}}{2 h E /\left(1-v^{2}\right)+\bar{\rho} c E}}
$$

where $c_{e l}^{(\text {para })} \approx \sqrt{\frac{2 h E /\left(1-v^{2}\right)+\bar{\rho} c E}{(2 h+\bar{\rho} c) \rho}}, P_{Y}^{(\text {para })}=\frac{2 h \sigma_{Y}}{\sqrt{1-v_{P}+v_{P}^{2}}}+\bar{\rho} c \sigma_{Y}, \quad \varepsilon_{Y}^{\prime}=\frac{P_{Y}^{(\text {para })}}{2 h E /\left(1-v^{2}\right)+\bar{\rho} c E}$.

The duration of front end reaction force curve at $\mathrm{P}^{(\mathrm{front})}$ is approximated as $\Delta t_{\text {pulse }}^{(\text {para })} \approx$ $L / 2 c_{p l}^{\text {(Para) }}$.

In Fig. 7.22, the analytic expressions for $\mathrm{P}^{\text {(front) }}$ and $\mathrm{P}^{(\mathrm{back})}$ described in Eqns.(7.8) and (7.9) are compared with FE results on the sandwich columns of varied face sheet thickness, h, with retaining the same core dimensions $(\bar{\rho}=0.25)$ and column length $(\mathrm{L}=469 \mathrm{~mm})$. The analytic expressions based on the theory of rate-independent wave propagaion agrees well with the FE results. 


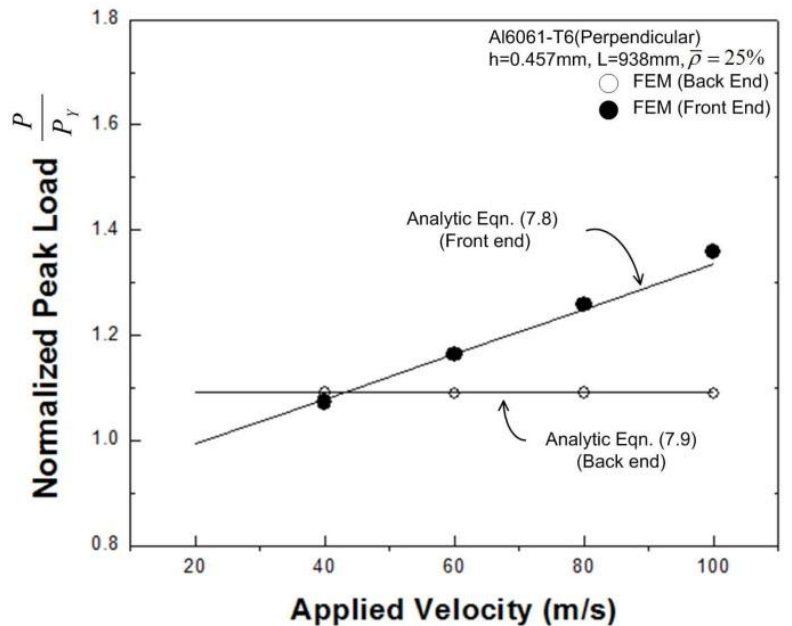

(a)

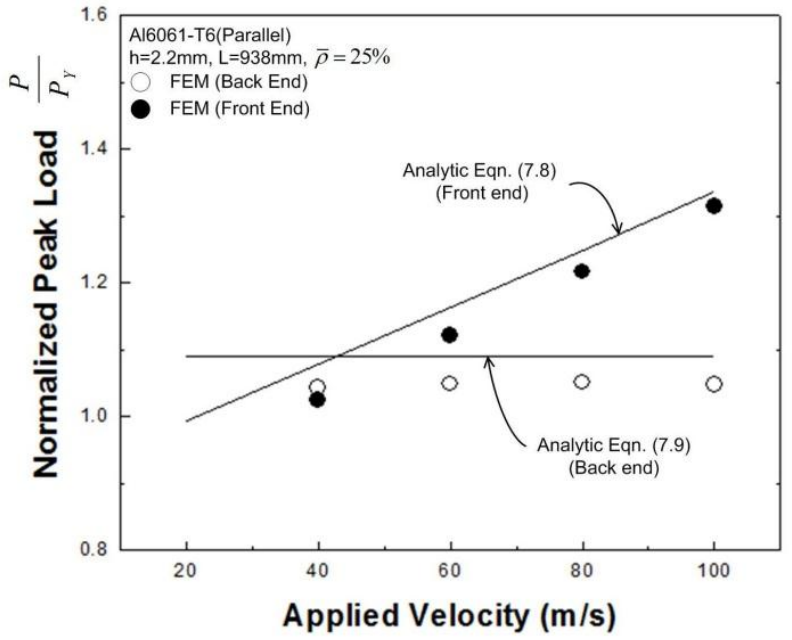

(b)

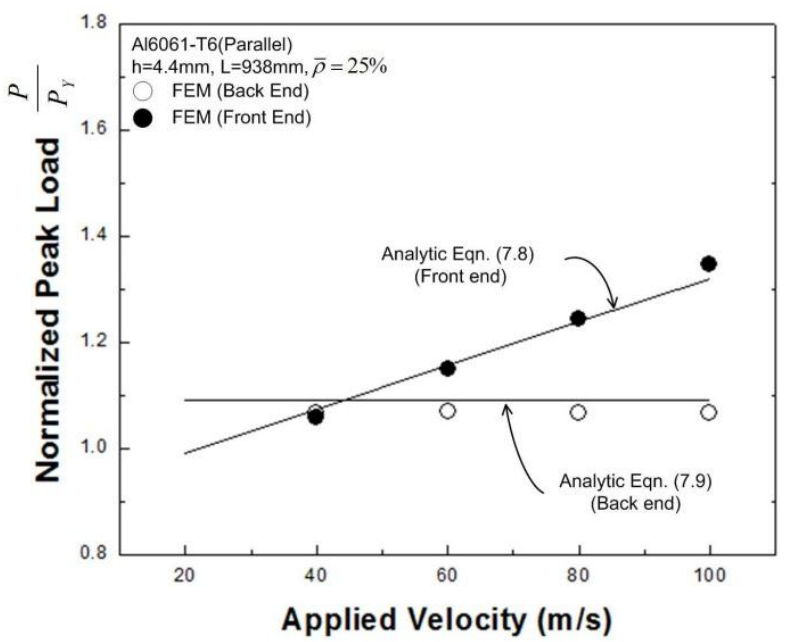

(c)

Figure 7.22: Comparison between analytic expressions and FE simulations of compression-velocity dependent peak reaction forces, $\mathrm{P}^{\text {(front) }}$ and $\mathrm{P}^{\text {(back) }}$, of Al6061-T6 sandwich columns having various face sheet thicknesses: (a) $\mathrm{h}=0.457 \mathrm{~mm}$; (b) $\mathrm{h}=2.2 \mathrm{~mm}$; (c) $\mathrm{h}=4.4 \mathrm{~mm}$. 


\subsubsection{Material Rate Dependence}

To this point in this section, the FE observations and analytic characterization focused on the rate-independent (Al6061-T6) corrugated core sandwich column compressed parallel-to-corrugations. In this subsection, how the material rate dependence is incorporated into the high velocity dynamic response is discussed through the FE analysis of SS304 corrugated core sandwich columns.

FE simulations are carried out for SS304 corrugated core sandwich columns of $\mathrm{h}=2.5 \mathrm{~mm}, \mathrm{~L}=707 \mathrm{~mm}$ subject to $\mathrm{V}=40 \mathrm{~m} / \mathrm{s}$ with and without considering material ratedependence. The FE simulation without material rate-dependence is also computed by suppressing the dynamic strengthening enhancement ratio, $R(\dot{p})$, in the parent material description. The reaction forces from the two FE computations are compared in Fig. 7.23. It is evident that the higher magnitude in the rate-dependent result attributes to the elevation in dynamic yield strength, i.e., the effects of material rate dependence.

It is important to note that the SS304 back end reaction force curves show significant hardening around $\mathrm{t}=700 \mu$ s compared to the Al6061-T6 columns. That is

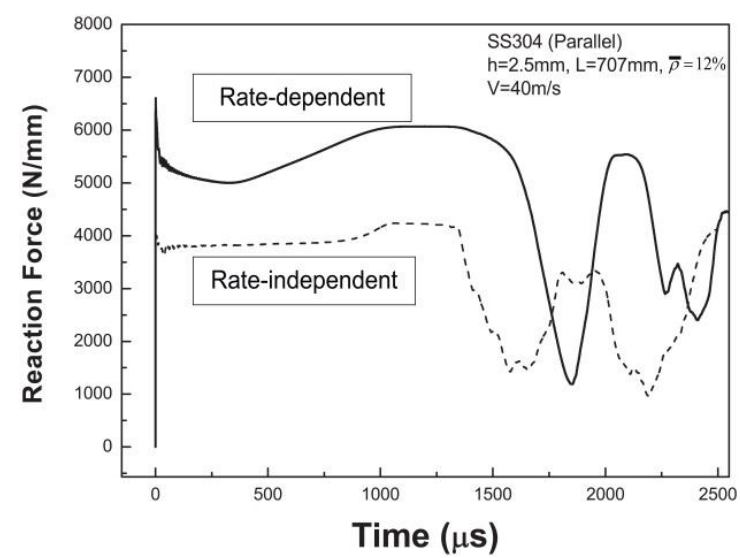

(a)

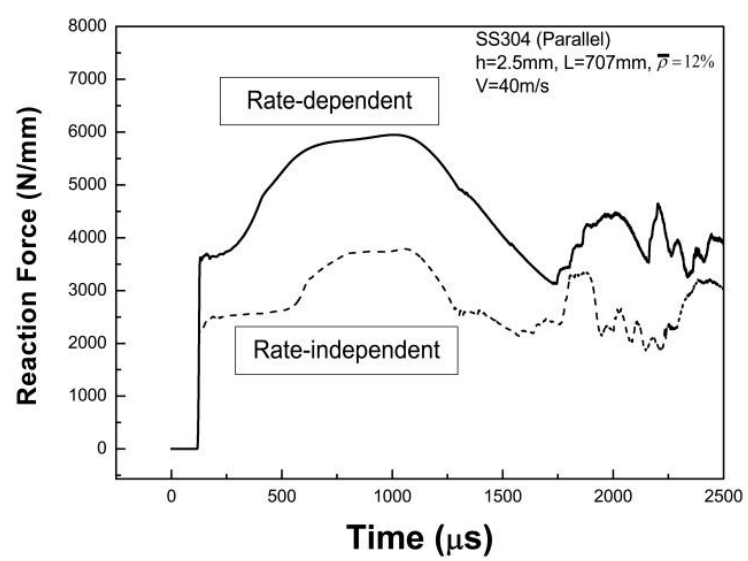

(b)

Figure 7.23: Material strain-rate dependence of SS304 corrugated core sandwich columns compressed parallel-to-corrugations: (a) Front end reaction force; (b) Back end reaction force. 


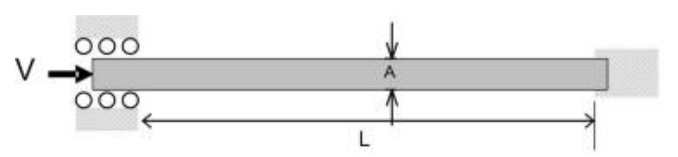

(a)

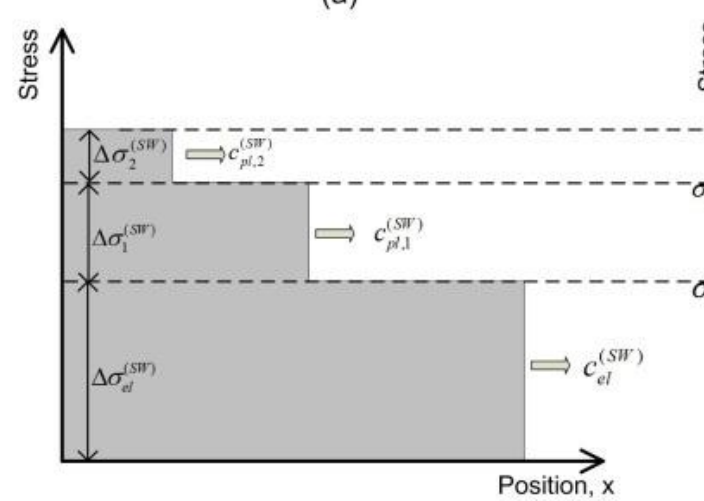

(b)

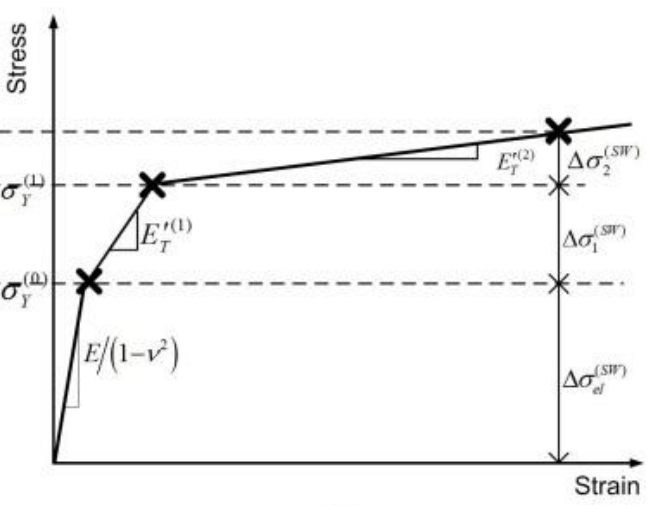

(c)

Figure 7.24: Three wave fronts propagated in a rate-independent elastic- plastic material with bilinear hardening moduli.

because the plastic part of SS304 is modeled by two plastic hardening moduli in the FE simulations, $E_{P}^{(1)}=\frac{d \sigma_{e q}}{d p}=8117.65(M P a)$ for small strain region $(0<\mathrm{p}<0.0164)$, and $E_{P}^{(2)}=\frac{d \sigma_{e q}}{d p}=2460.66(M P a)$ for large strain region $(\mathrm{p}>0.0164)$ as described in Fig. 7.13. In other words, the hardening response that emerged in the back end reaction force curves in Fig. 7.23(b) is explained by an arrival of the plastic wave associated with the first strain-hardening region whose corresponding stiffness and wave speed are $E_{P}^{(1)}$ and $c_{p l, 1}^{(S W)}$ as depicted in Fig. 7.24.

To examine if the rate-dependent result can be inferred from the rate-independent analysis, the rate-independent back and front end reaction curves scaled with $\mathrm{R}$ are compared to the rate-dependent results in Fig. 7.25. The two results, the rate-dependent FE reaction force curves and the R-scaled curves from the rate-independent FE curves, are similar. The only difference is that the hardening in the R-scaled curves appears stepwise while the rate-dependent FE results shows smooth increase in the hardening 


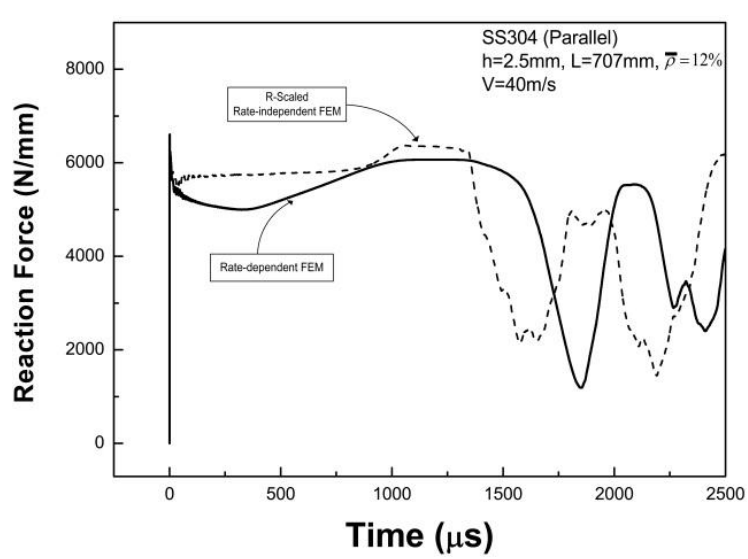

(a)

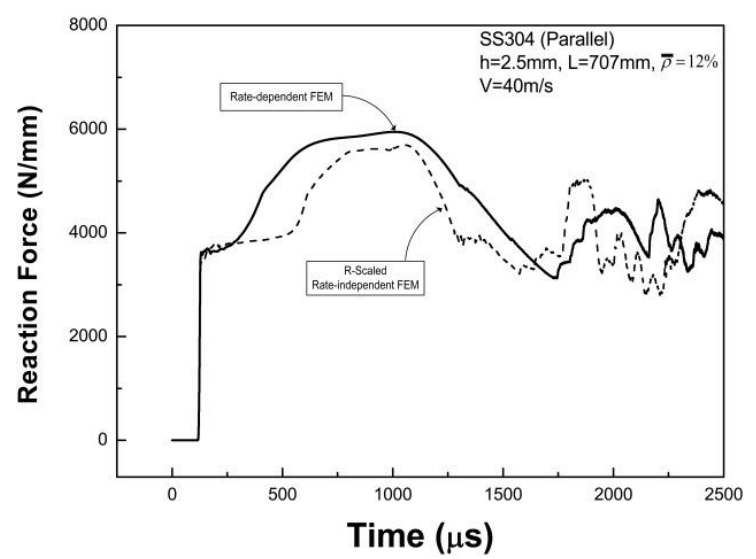

(b)

Figure 7.25: Comparison between the rate-dependent analysis and the scaled curves from the rate-independent analysis: (a) Front end reaction forces; (b) Back end reaction forces.

region. This phenomenon can be explained by the findings from the two independent studies, Ferri [3] and Garnet [72] work: The shape of wave fronts travelling along a ratedependent column is indistinct. As a result, associated plastic strain distribution is quite dispersed [3]. On the other hand, the distinct wave fronts are observed so that the stressstrain distribution over a column is spatially stepwise, and thus, reaction force curve histories appear stepwise $[3,72]$ as in the primary assumption of this dissertation.

Nevertheless, the rate-dependent response is reasonably approximated from scaling the rate-independent results. Therefore, it can be concluded that the response of a ratedependent material under high velocity compression is approximately predicted from a rate-independent analysis of treating the rate-dependent material as a rate-independent material having R-scaled yield stress and tangent modulus.

As in Section 7.3.4, the peak forces considering the material strain-rate dependence of a parent material whose plastic part is modeled by two plastic hardening moduli can be obtained just by simply multiplying the rate-independent expressions by $R(\dot{\mathrm{p}})$. 


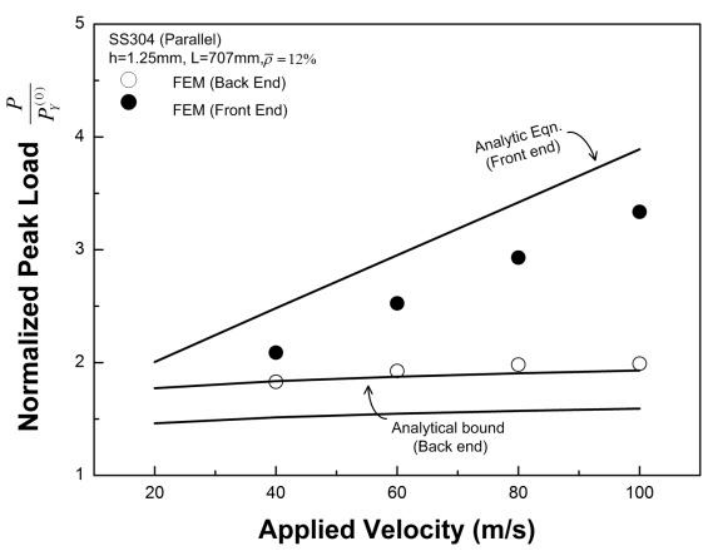

(a)

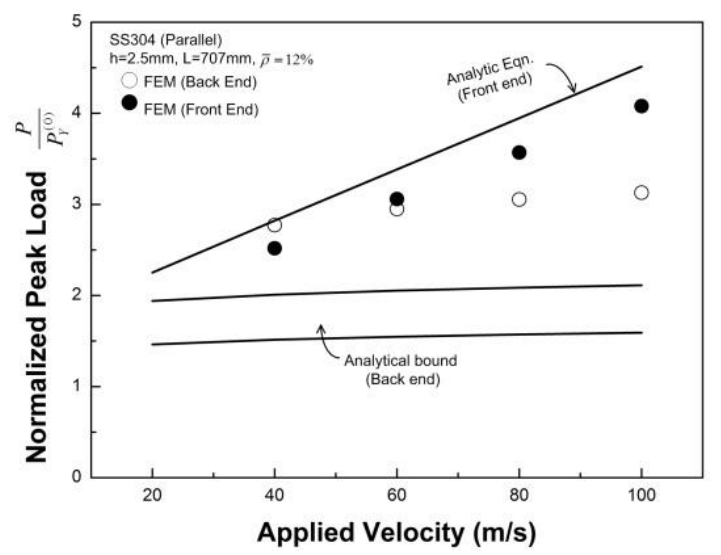

(b)

Figure 7.26: Analytic prediction of peak reaction forces of rate-dependent (SS304) corrugated core sandwich columns under high velocity compression parallel-to-corrugations: (a) h=1.25mm; (b) h=2.5mm.

$$
\begin{gathered}
\frac{P^{(\text {front })}}{P_{Y}^{(0)}} \mid \approx R(\dot{p}) \cdot\left[\frac{P_{Y}^{\prime(1)}}{P_{Y}^{\prime(0)}}+\sqrt{\frac{2 h E_{T}^{\prime(2)}+\bar{\rho} c E_{T}^{(2)}}{2 h E /\left(1-v^{2}\right)+\bar{\rho} c E}}\left(\frac{V}{c_{e l}^{(0)(\text { para })} \varepsilon_{Y}^{\prime(0)}}-1\right)-\sqrt{\left.\frac{2 h E_{T}^{\prime(2)}+\bar{\rho} c E_{T}^{(2)}}{2 h E_{T}^{\prime(1)}+\bar{\rho} c E_{T}^{(1)}} \frac{\left.P_{Y}^{\prime(1)}-P_{Y}^{\prime(0)}\right)}{P_{Y}^{\prime(0)}}\right](7 .}\right] \\
\frac{P_{\text {upper }}^{(\text {back })}}{P_{Y}^{\prime(0)}} \approx R(\dot{p}) \cdot\left[\frac{P_{Y}^{\prime(1)}}{P_{Y}^{\prime(0)}}+\sqrt{\frac{2 h E_{T}^{\prime(2)}+\bar{\rho} c E_{T}^{(2)}}{2 h E /\left(1-v^{2}\right)+\bar{\rho} c E}}-\sqrt{\frac{2 h E_{T}^{\prime(2)}+\bar{\rho} c E_{T}^{(2)}}{2 h E_{T}^{\prime(1)}+\bar{\rho} c E_{T}^{(1)}}} \cdot \frac{P_{Y}^{\prime(1)}-P_{Y}^{\prime(0)}}{P_{Y}^{\prime(0)}}\right] \\
\frac{P_{\text {lower }}^{(\text {back })}}{P_{Y}^{\prime(0)}} \approx R(\dot{p})
\end{gathered}
$$

where $\varepsilon_{Y}^{\prime(0)}=\frac{P_{Y}^{\prime(0)}}{2 h E /\left(1-v^{2}\right)+\bar{\rho} c E}, P_{Y}^{\prime(k)}=2 h \frac{\sigma_{Y}^{(k)}}{\sqrt{1-v_{P}+v_{P}^{2}}}+\bar{\rho} c \sigma_{Y}^{(k)}, c_{e l}^{(0)} \approx \sqrt{\frac{2 h E /\left(1-v^{2}\right)+\bar{\rho} c E}{(2 h+\bar{\rho} c) \rho}}$.

In Fig. 7.26, the analytic expressions corrected for the inclusion of the material strain-rate effects are compared with FE calculations of SS304 corrugated core sandwich columns of $\mathrm{h}=1.25,2.5 \mathrm{~mm}$, respectively. 


\subsection{CHAPTER SUMMARY}

This chapter investigates the dynamic response of corrugated core sandwich columns subject to a velocity in the high velocity region via FE simulations. The peak and duration in reaction forces are parameterized with respect to compression velocities, material properties, and sandwich geometric parameters utilizing the theory of rateindependent elastic-plastic wave propagation.

The responses in the high velocity range differs from the low-velocity response in that the response time scale is of the order of a one-way trip time of an plastic stress wave along the column length.

In the perpendicular-to-corrugation loading orientation, face wrinkling dominates the structural response. The face sheet ligaments closest to the front end exhibit face wrinkling before an incident plastic wave passes one unit cell under the intense loading situation. Macroscopically, contact forces between face and core members from progressive densification of unit cells are repeatedly observed. The back end reaction forces are determined by the face wrinkling failure of the face ligament closest to the back end due to the incident elastic wave and its reflected plastic wave. The parameters for the front and back end reaction forces in the in-plane loading orientation are well predicted by the modified analytic expressions suggested in this chapter.

In the parallel-to-corrugation loading orientation, the failure leading to load drop is the result of global buckling motion when the two plastic waves meet and superpose in the mid-section of the column. The high velocity response of corrugated core sandwich columns compressed parallel-to-corrugations is also well-characterized by the analytic expressions based on the theory of wave propagation. 


\section{CHAPTER 8}

\section{CONCLUDING REMARKS}

\subsection{SUMMARY}

Theoretical models are developed to predict the dynamic response of corrugated core sandwich columns under a compression velocity in the low velocity region (from quasi-static to an order of a few $\mathrm{m} / \mathrm{s}$ ). Each model reproduces the dynamic explicit $\mathrm{FE}$ simulations of the individual failure modes such as global buckling, face wrinkling, and local plate buckling. Not only do the theoretical models combined with the FEM analyze the dominant dynamic effects but also the influences on the minimum weight designs and competing failure modes are identified.

The global buckling analytical model considers a sandwich column as a beamcolumn structural element with core shear deformations. The model successfully yields the imperfection-sensitive, velocity-dependent dynamic response and appearance of higher buckling modes. In addition to the transverse-inertia dominating dynamic strengthening (i.e., inertial stabilization), the material strain-rate dependent response is reasonably approximated for the SS304 corrugated core sandwich column response. It is also found that imperfections influence the global buckling motion of elastic columns, whereas their effect on the elastic-plastic global column response is not as significant as with the elastic column response.

The face wrinkling analytical model calculates a face ligament transverse deflection of a face ligament modeled by an Euler-Bernoulli beam-column using continuum 
approximation of the discrete cores with wave propagation theory. A fundamental assumption in the face wrinkling analytical model is verified by numerical experiments for measuring the apparent elastic wave propagation speeds. It is noteworthy that the apparent wave speeds depend not only on material properties but also on sandwich design parameters unlike monolithic solid columns. The numerical verification of the model successfully captures the dynamic phenomena observed in the FE simulations including dynamic strengthening, material strain-rate effects and imperfection sensitivity. However, there is a limitation that the model is not capable of predicting the plateau response which has been seen in the representative Al6061-T6 sandwich columns of the EFW geometric dimensions, especially compressed at a low velocity. It is demonstrated that the plateau response results from local plastic deformation at nodes.

An attempt to approximate the local plate buckling response of sandwich columns compressed parallel-to-corrugations was made through developing an analytical model for long plates. The analytical model assumes that the structural integrity of the sandwich columns is determined from the occurrence and growth of a buckle in the imaginary patch plates. The analytical prediction for the elastic plate response at $\mathrm{V}=1 \mathrm{~m} / \mathrm{s}$ was wellcorrelated with the FE calculations. However, the post critical response characterized by a reduced stiffness at a low velocity of $\mathrm{V}=0.1 \mathrm{~m} / \mathrm{s}$ and elastic-plastic plate buckling could not be reproduced.

The developed theoretical models are quite attractive at the point of efficiency with a reasonable accuracy. A combination of global buckling and local buckling analytical models can be utilized in numerous potential applications such as preliminary sandwich column designs. For example, the competition of failure modes under dynamic 
circumstances and minimum weight design considering dynamic effects are investigated with those developed analytical models in this dissertation.

In the dynamic failure map analysis, dynamic effects on the two quasi-static failure maps of corrugated core sandwich columns compressed perpendicular-to-corrugations are analyzed as rate-of-compression increases. In the investigation, operative failure modes can be identified by comparing the times-to-failure computed from the global and face wrinkling analytical models. It is demonstrated that inertial stabilization of global buckling motion makes the global buckling motion more resistible than face wrinkling as rate-of-compression increases. Furthermore, the effect of imperfections on the failure map boundary between FY and EGB becomes more pronounced with increase in rate-ofcompression. The key contribution in the analysis is that how compression velocities and imperfections alter the failure boundaries is identified.

The efficiency of the developed analytical models is highlighted in the dynamic optimization procedure. Because there is no explicit expression for the dynamic response in terms of design variables, a methodology for corrugated core sandwich columns is provided. Due to the complex dynamic phenomena, the individual failure responses are approximated through a number of numerical experiments for the response range of interest. Thus, a response surface methodology is employed for the response approximation of individual failure modes, and one of classical optimal solution algorithms (Sequential Quadratic Programming) for minimum weight designs considering the dynamic effects is also employed. The optimal solutions demonstrate that optimal designs of corrugated core sandwich columns under quasi-static loading and under dynamic loading differ in that global buckling motion is influenced more by 
beneficial inertial stabilization than by the face wrinkling motion and that sandwich columns are superior to monolithic solid columns under dynamic loading; however, the benefit of sandwich design concept is even more remarkable at lower velocities. Those findings are consistent with the results of dynamic failure map analysis.

The dynamic response subject to a velocity in the high velocity region, $V /\left(c_{e l}^{(S W)} \varepsilon_{Y}\right)>1$, is also investigated via FE simulations. The responses in the high velocity range differ from the low-velocity region response in that the response time scale is of the order of a one-way trip time of a plastic wave along the column length. Thus, the peak and duration in reaction forces are parameterized with respect to compression velocities, material properties, and sandwich geometric parameters utilizing the theory of rate-independent elastic-plastic wave propagation.

\subsection{ORIGINAL CONTRIBUTIONS}

1. The development of analytical models for the individual failure models in corrugated core sandwich columns. Since there have been no analytical tools predicting the dynamic response of individual failure modes to date, the models make possible the dynamic effect assessment of individual failure modes. Furthermore, emphasis is placed on the following two aspects: wide applicable compression velocity and the efficiency. Because the models consider non-uniform stress distribution due to wave propagation, they can be applied to a relatively large magnitude of compression velocities. And, the models can also reproduce the FE simulations requiring a large number of elements for periodic core structures with limited computing time. 
2. Dynamic strengthening caused from transverse inertia, material strain-rate effects etc. are understood through the FE and theoretical investigations. In addition, the combined effect of imperfections with the dynamic loading is also investigated.

3. A dynamic optimization methodology is introduced into the area of metallic core sandwich structures. In this dissertation, a dynamic optimization problem is established with response surface methodology, in which complex dynamic response is represented by easily manageable polynomial functions.

The RSM is employed in many engineering fields, especially in cases where many design variables or complicated phenomena make it difficult to find an accurate function for the response. Instead, RSM seeks to find easy-to-handle polynomial functions to fit a set of numerical or experimental design points in a carefully planned design of experiments (e.g., full-factorial DOE, DOE using an orthogonal array table, etc.). For example, designing safety components for frontal crash in the crashworthiness area or determining optimal operating conditions in Direct Chill casting are good examples for application of the methodology. Because the dynamic response of corrugated core sandwich column cannot be explicit in terms of their geometric parameters, the optimization procedure for minimum weight designs considering dynamic effects is appropriate.

4. High velocity response of corrugated core sandwich columns is generalized with respect to the intensity of compression velocity and sandwich design parameters in this dissertation. This will provide insight for a design guideline of a structural component with minimum weight exposed to intense loading such as blast loading. 


\subsection{FUTURE WORK}

Several points are noted for the extension of this study.

First, it was assumed that the failure of corrugated core sandwich columns was caused only by buckling in this dissertation. However, there is a possibility that other failure mechanisms exist. For example, the sandwich columns may not function effectively due to a fracture at fixed ends, cracking or delamination at nodes between cores and face sheets. Those failures are presumably due to a lack of material ductility, stress concentration, and inadequate manufacturing, etc. In this regard, the theoretical model can be extended considering such failure mechanisms.

Second, the fidelity of analytical models was justified by comparing to the FEM, which is generally known to be an accurate numerical tool. However, the gaps between theoretical/numerical results and experiments might be unexpectedly large. Thus, an experimental verification for each failure mode, together with the discussion of potential discrepancy, would reinforce the justification of the developed analytical models and observed dynamic phenomena.

Third, minimum weight designs including dynamic effects are discussed as the rateof-compression is increased in this dissertation. It is instructive that the reinforcement of local buckling strength is suggested under dynamic circumstances. However, if multifunctional capability, the distinguished advantage of metallic core sandwich structures, is reflected in the optimal design of sandwich structures, the optimization results will be different. Multi objective optimization of metallic core sandwich structures under dynamic loading with the second function such as blast loading energy absorption, ballistic energy resistance, and a flow channel, can be the next step. 


\section{REFERENCES}

[1] Wadley, H. N. G. Multifunctional periodic cellular metals. Philosophical Transactions of the Royal Society A: Mathematical, Physical and Engineering Sciences 364, (2006) 31-68.

[2] Evans, A. G., Hutchinson, J. W., Fleck, N. A., Ashby, M. F., and Wadley, H. N. G. The topological design of multifunctional cellular metals. Progress in Materials Science 46, 3-4 (2001), 309-327.

[3] Ferri, E., Antinucci, E., He, M.Y., Hutchinson, J. W., Zok, F.W. and Evans, A.G. Dynamic buckling of impulsively loaded prismatic cores, Journal of Mechanics of Materials and Structure 1, 8 (2006) 1345-1365.

[4] Tilbrook, M.T., Radford, D.D., Deshpande, V.S, Fleck, N.A. Dynamic crushing of sandwich panels with prismatic lattice cores, International Journal of Solids and Structures 44, 18-19 (2007) 6101-6123.

[5] Radford, D.D., McShane, G.J., Deshpande, V.S., Fleck, N.A. Dynamic compressive response of stainless-steel square honeycombs. Journal of Applied Mechanics 74, 4 (2007) 658-667.

[6] Lee, S., Barthelat, F., Hutchinson, J.W., Espinosa, H.D. Dynamic failure of metallic pyramidal truss core materials-Experiments and modeling. International Journal of Plasticity 22, 11 (2006) 2118-2145.

[7] Rubino, V., Deshpande, V.S., and Fleck, N.A. The dynamic response of end clamped sandwich beams with a Y-frame or corrugated core. International Journal of Impact Engineering 35, 8 (2008) 829-844.

[8] Rubino, V., Deshpande, V.S., and Fleck, N.A. The dynamic response of clamped rectangular Y-frame and corrugated core sandwich plates. European Journal of Mechanics A-Solids 28, 1 (2009) 14-24.

[9] Radford, D.D., Fleck, N.A. and Deshpande, V.S. The response of clamped sandwich beams subjected to shock loading. International Journal of Impact Engineering 32, 6 (2006) 968-987.

[10] Tilbrook, M.T., Deshpande, V.S., and Fleck, N.A. The impulsive response of sandwich beams: Analytical and numerical investigation of regimes of behaviour. Journal of the Mechanics and Physics of Solids 54, 11 (2006) 2242-2280.

[11] Qiu, X., Deshpande, V.S., Fleck, N.A. Impulsive loading of clamped monolithic and sandwich beams over a central patch. Journal of the Mechanics and Physics of Solids 53, 5 (2005) 1015-1046.

[12] Mori, L.F., Queheillalt, D.T., Wadley, H.N.G., Espinosa, H.D. Deformation and 
failure modes of I-core sandwich structures subjected to underwater impulsive loads. Experimental Mechanics 49, 2 (2009) 257-275.

[13] Mori, L.F., Lee, S., Xue, Z.Y., Vaziri, A., Queheillalt, D.T., Dharmasena, K.P., Wadley,H.N.G., Hutchinson, J.W., Espinosa, H.D. Deformation and fracture modes of sandwich structures subjected to underwater impulsive loads. Journal of Mechanics of Materials and Structures 2, 10 (2007) 1981-2005.

[14] Wadley, H.N.G., Dharmasena, K.P., Queheillalt, D.T., Chen, Y., Dudt, P., Knight, D., Kiddy, K., Xue, Z., Vaziri, A. Dynamic compression of square honeycomb structures during underwater impulsive loading., Journal of Mechanics of Materials and Structures 2, 10 (2007) 2025-2048.

[15] Wei, Z., Dharmasena, K.P., Wadley, H.N.G. and Evans. A.G. Analysis and interpretation of a test for characterizing the response of sandwich panels to water blast. International Journal of Impact Engineering 34, 10 (2007) 1602-1618.

[16] McShane, G.J., Deshpande, V.S. and Fleck, N.A. The underwater blast resistance of metallic sandwich beams with prismatic lattice cores. Journal of Applied Mechanics 74, 2 (2007) 352-364.

[17] McMeeking, R.M., Spuskanyuk, A.V., He, M.Y., Deshpande, V.S., Fleck, N.A., Evans, A.G. An analytic model for the response to water blast of unsupported metallic sandwich panels. International Journal of Solids and Structures 45, 2 (2008) 478-496.

[18] Rathbun, H.J., et al. Performance of metallic honeycomb-core sandwich beams under shock loading. International Journal of Solids and Structures 43, 6 (2006) 17461763.

[19] Tagarielli, V.L., Deshpande, V.S., and Fleck, N.A., The dynamic response of composite sandwich beams to transverse impact. International Journal of Solids and Structures 44, 7-8 (2007) p. 2442-2457.

[20] Fleck, N. A. and Deshpande, V.S. The resistance of clamped sandwich beams to shock loading. Journal of Applied Mechanics 71, 3 (2004) 386-401.

[21] Fleck, N.A., Tagarielli, V.L. and Deshpande, V.S. The dynamic response of composite sandwich beams to transverse impact. International Journal of Solids and Structures 44, 7-8 (2007) 2442-2457.

[22] Qiu, X., Deshpande, V.S., Fleck, N.A. Finite element analysis of the dynamic response of clamped sandwich beams subject to shock loading. European Journal of Mechanics A-Solids 22, 6 (2003) 801-814.

[23] Liang, Y. et al. The response of metallic sandwich panels to water blast. Journal of Applied Mechanics 74, 1 (2007) 81-99.

[24] Xue, Z., Hutchinson, J.W. A comparative study of impulse-resistant metal sandwich 
plates. International Journal of Impact Engineering 30, 10 (2004) 1283-1305.

[25] Vaughn,D.G. and Hutchinson, J.W. Bucklewaves. European Journal of Mechanics A/Solids 25, 1 (2006) 1-12.

[26] Ji, W. Static and dynamic response of a sandwich structure under axial compression. Ph.D Dissertation, University of Michgan, Ann Arbor, USA, 2008

[27] Biagi, R., Lim, J., Bart-Smith, H. In-Plane compression response of extruded aluminum 6061-T6 corrugated core sandwich columns. Journal of the American Ceramic Society 94, S1 (2011) 76-84.

[28] Biagi, R. The mechanical response of corrugated core sandwich columns. Ph.D Dissertation, University of Virginia, Charlottesville, USA, 2010.

[29] Cote, F., Biagi, R., Bart-Smith, H. Deshpande, V.S. Structural response of pyramidal core sandwich columns. International Journal of Solids and Structures 44, 10 (2007) 3533-3556.

[30] Lindberg, H. E., and Florence, A. L. Dynamic Pulse Buckling: Theory and Experiment. Martinus Nijhoff, Norwell, MA, 1987.

[31] Zhang, Q., Li, S., Zheng, J. Dynamic response, buckling and collapsing of elasticplastic straight columns under axial solid-fluid slamming compression I. Experiments. International Journal of Solids and Structures 29, 3 (1992) 381-397.

[32] Simitses, G.J. Dynamic stability of suddenly loaded structures. Springer-Verlag, New York Inc., 1990.

[33] Jones, N. Structural Impact. Cambridge University Press, Cambridge, 1989.

[34] Lindberg, H.E. Impact buckling of a thin bar. Journal of Applied Mechanics 32, 2 (1965) 315-322.

[35] Lepik, U. A contribution to bifurcation analysis of elastic-plastic beams. International Journal of Impact Engineering 21, 1-2 (1999) 35-49.

[36] Lepik, U. Dynamic buckling of elastic-plastic beams including effects of axial stress waves. International Journal of Impact Engineering 25, 6 (2001) 537-552.

[37] Lepik, U. On dynamic buckling of elastic-plastic beams. International Journal of Non-Linear Mechanics 35, 4 (2000) 721-734.

[38] Hoff, N.J. The dynamics of the buckling of elastic columns. Journal of Applied Mechanics 18, 1 (1951) 68-74.

[39] Sevin, E. On the bending of columns due to dynamic axial forces including effects of axial inertia. Journal of Applied Mechanics 27, 1 (1960) 125-131. 
[40] Lee, L. H. N. Quasi-bifurcation of rods within an axial plastic compressive wave. Journal of Applied Mechanics 45, 1 (1978) 100-104.

[41] Lee, L. H. N. Dynamic buckling of an inelastic column. International Journal of Solids and Structures 17, 3 (1981) 271-279.

[42] Lee, L. H. N. Flexural waves in rods within an axial plastic compressive wave. Wave Motion 3, 3 (1981) 243-255.

[43] Ari-Gur, J., Weller, T., Singer, J. Experimental and theoretical studies of columns under axial impact. International Journal of Solids and Structures 18, 7 (1982) 619-641.

[44] Ari-Gur, J., Eliashakoff, I., Dynamic instability of a transversely isotropic column subjected to a compression pulse, Computers \& Structures 62, 5 (1997) 811-815.

[45] Havashi, T., Sano, O. Dynamic buckling of elastic bars (1st report : The case of low velocity impact). Bulletin of the JSME 15, 88 (1972) 1167-1175.

[46] Havashi, T., Sano, O. Dynamic buckling of elastic bars (1st report: The case of high velocity impact). Bulletin of the JSME 15, 88 (1972) 1176-1184.

[47] Ji, W., Waas, A.M. Dynamic bifurcation buckling of an impacted column. International Journal of Engineering Science 46, 10 (2008) 958-967.

[48] Kenny, S., Taheri, F., Pegg, N. Finite element investigations on the dynamic plastic buckling of a slender beam subject to axial impact. International Journal of Impact Engineering 27, 2 (2002) 179-195.

[49] Kenny, S., Taheri, F., Pegg, N. Experimental investigations on the dynamic plastic buckling of a slender beam subject to axial impact. International Journal of Impact Engineering 27, 1(2002) 1-17.

[50] Abrahamson, G. R. and Goodier, J. N. Dynamic flexural buckling of rods within an axial plastic compression wave. Journal of Applied Mechanics 33, 2 (1966) 241-248.

[51] Vaughn, D. G., Canning, J. M., and Hutchinson, J. W. Coupled plastic wave propagation and column buckling. Journal of Applied Mechanics 72, 1 (2005) 139-146.

[52] Bell, J. F. The dynamic buckling of rods at large plastic strain. Acta Mechanica 74, $1-4$ (1988) 51-67.

[53] Sugiura, K., Mizuno, E. and Fukumoto, Y. Dynamic instability analyses of axially impacted columns. Journal of Engineering Mechanics 111, 7 (1985) 893-908.

[54] Cui, S., Cheong, H.K., Hao, H. Dynamic buckling of simply supported columns under axial slamming. Journal of Engineering Mechanics 125, 5 (1999) 513-520.

[55] Cui, S., Cheong, H.K., Hao, H. Experimental study of dynamic buckling of plates 
under fluid-solid slamming. International Journal of Impact Engineering 22, 7 (1999) 675-691.

[56] Cui, S., Hao, H., Cheong, H.K. Theoretical study of dynamic elastic buckling of columns subjected to intermediate velocity impact loads. International Journal of Mechanical Sciences 44, 4 (2002) 687-702.

[57] Hao, H., Cheong, H.K., Cui, S. Analysis of imperfect column buckling under intermediate velocity impact. International Journal of Solids and Structures 37, 38 (2000) 5297-5313.

[58] Cui, S., Cheong, H.K., Hao, H. Experimental study of dynamic post-buckling characteristics of columns under fluid-solid slamming. Engineering Structures 22, 6 (2000) 647-656.

[59] Cui, S., Hao, H., Cheong, H.K. Dynamic buckling and post-buckling of imperfect columns under fluid-solid interaction. International Journal of Solids and Structures 38, 48 (2001) 8879-8897.

[60] Su, X.Y., Yu, T.X., Reid, S.R. Inertia-sensitive impact energy-absorbing structures part 1: effects of inertia and elasticity. International Journal of Impact Engineering 16, 4 (1995) 651-672.

[61] Su, X.Y., Yu, T.X., Reid, S.R. Inertia-sensitive impact energy-absorbing structures part 2: effect of strain rate. . International Journal of Impact Engineering 16, 4 (1995) 673-689.

[62] Calladine, C.R., English, R.W. Strain-rate and inertia effects in the collapse of twotypes of energy absorbing structures. International Journal of Mechanical Sciences 26, 11-12 (1984) 689-701.

[63] Karagiozova D, Jones N. Multi-degrees of freedom model for dynamic buckling of an elastic-plastic structure. International Journal of Solids and Structures 33, 23 (1996) 3377-3398.

[64] Bazant, Z.P. and Cedolin, L.. Stability of structures : elastic, inelastic, fracture and damage theories. Oxford University Press, New York, 1991.

[65] Allen, H.G. Analysis and design of structural sandwich panels. Pergamon Press, London, 1969.

[66] Zenkert, D. An introduction to sandwich construction. Engineering Materials Advisory Services, London, 1995.

[67] Plantema, F.J. Sandwich construction. Wiley, New York, 1966. 
[68] Côté, F., Deshpande, V.S., Fleck, N. A., Evans, A.G. The compressive and shear responses of corrugated and diamond lattice materials. International Journal of Solids and Structures 43, 20 (2006) 6220-6242.

[69] Becque, J. Inelastic plate buckling, Journal of Engineering Mechanics 136, 9 (2010) 1123-1130.

[70] von Karman,T., Duwez, P. The propagation of plastic deformation in solids. Journal of Applied Physics 21, 10 (1950) 987-994.

[71] Taylor, G. I. The plastic wave in a wire extended by an impact load. The Scientific Papers of G. I. Taylor Vol. 1 Mechanics of Solids edited by G. K. Batchelor, University Press, Cambridge, England, (1958) 467-479.

[72] Garnet, H. and Armen, H. One dimensional elasto-plastic wave interaction and boundary reflections. Computers and Structures 5, 5-6 (1975) 327-334.

[73] Stronge, W.J. Impact mechanics. University Press, Cambridge, England, 2000.

[74] Lee, H.P. Effects of initial curvature on the dynamic stability of a beam with tip mass subjected to axial pulsating loads. International Journal of Solids and Structures 32, 23 (1995) 3377-3392.

[75] Perrone, N. On a simplified method for solving impulsively loaded structures of rate-sensitive materials. Journal of Applied Mechanics 32, 3 (1965) 489-492.

[76] Maiden, C.J. and Green, S.J. Compressive strain-rate tests on six selected materials at strain rates from $10^{-3}$ to $10^{4} \mathrm{in} / \mathrm{in} / \mathrm{s}$., Journal of Applied Mechanics 33, 3 (1966) 496504.

[77] Lindholm, U.S. Some experiments with the split-Hopkinson pressure bar. Journal of the Mechanics and Physics of Solids 12, 5 (1964) 317-335.

[78] Stout, M.G., and Follansbee, P.S. Strain rate sensitivity, strain hardening and yield behavior of 304L stainless steel. Journal of Engineering Materials Technology 108, (1986) 344-353.

[79] Cowper, G.R. and Symonds, P.S. Strain hardening and strain rate effects in the impact loading of cantilever beams, Brown University Division of Applied Mathematics Report No. 28, September 1957.

[80] Haberman, R. Applied partial differential equations, $4^{\text {th }}$ edition, Prentice Hall, 2003.

[81] Timoshenko, S.P. and Woinowski-Krieger, S. Theory of Plates and Shells, (2nd Edition). McGraw-Hill Kogakusha, 1959. p. 393-395.

[82] Baber, T. Lecture Notes on Plates and Shells, Charlottesville. University of Virginia, 2011. 
[83] Shames, I.H. and Dym, C.L. Energy and Finite Element Methods in Structural Mechanics, Taylor and Francis, New York, 1985.

[84] Bleich, F. Buckling Strength of Metal Structures. McGraw-Hill, New York, 1952.

[85] Ros, M., and Eichinger, A. Final Rep. of the 1st Congress. International Association of Bridge and Structural Engineering 144, Paris, 1932.

[86] Gerard, G. Secant modulus method for determining plate instability above the proportionality limit. Journal of Aeronautical Sciences 13, 1 (1946) 38-44.

[87] Fleck, N.A. and Sridhar, I. End compression of sandwich columns. Composites: Part A 33, 3 (2002) 353-359.

[88] Tam, L.L., Calladine, C.R. Inertia and strain-rate effects in a simple plate-structure under impact loading. International Journal of Impact Engineering 11, 3 (1991) 349-377.

[89] Budiansky, B. On the minimum weight of compression members. International Journal of Solids Structures 36, (1999) 3677-3708.

[90] Tian, Y.S. and Lu, T,J. Optimal design of compression corrugated panels. Thinwalled Structures 43, 3 (2005) 477-498.

[91] Wicks, N. and Hutchinson, J.W. Optimal truss plates. International Journal of Solids Structures 38, 30-31 (2001) 5165-5183.

[92] Montgomery, D.C. Design and Analysis of Experiments 5th Edition. John Wiley, New York, 2000.

[93] Arora, J.S. Introduction to optimum design. Academic Press, 2004.

[94] Belegundu, A.D. and Chandrupatla, T.R. Optimization concepts and applications in engineering. Prentice Hall, Upper Saddle River, N.J., 1999.

[95] Altair Product Hyperstudy v10.0 Theory and User Manual.

[96] Balawi, Effective Mechanical Behavior of honeycombs theoretical and Experimental studies, p20, Section 2.3.2, University of Cincinatti, 2007..

[97] Khan, A.S. and Huang, S. Continuum Theory of Plasticity. John Wiley, New York, 1995.

[98] Bloom, F., and Coffin, D. Handbook of Thin Plate Buckling and Postbuckling. Chapman \& Hall, Boca Raton, FL, 2000. 
[99] Qiu, X., Deshpande, V.S., Fleck, N.A. Dynamic response of a clamped circular sandwich plate subject to shock loading. Journal of Applied Mechanics 71, 3 (2004) 637645.

[100] Reddy, J.N. Mechanics of Laminated Composite Plates: Theory and Analysis. CRC Press, Boca Raton, 2004.

[101] Vinson, J.R. The Behavior of Sandwich Structures of Isotropic and Composite Materials. CRC Press, Lancaster, 1999.

[102] Zhang, Z., Liu, S., Tang, S. Crashworthiness investigation of Kagome honeycomb sandwich cylindrical column under axial crushing loads. Thin-walled Structures 48 (2010) 9-18.

[103] Biagi, R., Bart-Smith, H. In-plane column response of metallic corrugated core sandwich columns. International Journal of Solids and Structures (in press).

[104] Gama, B.A., Lopatnikov, S.L., Gillespie, J.R. Hopkinson bar experimental technique: A critical review. Applied Mechanics Reviews 57, 4 (2004) 223-250.

[105] van Hoof, J.F.A.M. One- and two dimensional wave propagations in solids. WFWreport 94.055, Eindhoven University of Technology, the Netherlands, 1994.

[106] Chree, C. The equations of an isotropic elastic solid in polar and cylindrical coordinates, their solution and applications. Trans. Cambridge Philos. Soc. 14, (1889) $251-369$.

[107] Belytschko, T., Liu, W.K., Moran, B., Nonlinear Finite Elements for Continua and Structures. John Wiley \& Sons, New York, 2000. 


\section{APPENDIX A DERIVATION OF EQUATIONS (2.30) AND (2.31).}

$$
\begin{gathered}
\frac{\partial P(x, t)}{\partial x}=\rho A \frac{\partial^{2} u^{(m)}}{\partial t^{2}} \\
P(x, t)=(A E)^{*} \frac{\partial u^{(m)}}{\partial x}-\frac{1}{2}(A E)^{*}\left[\left(\frac{\partial\left(w^{(m)}+w_{0}^{(m)}\right)}{\partial x}\right)^{2}-\left(\frac{\partial w_{0}^{(m)}}{\partial x}\right)^{2}\right]
\end{gathered}
$$

Substituting (2.22) into (2.3), we will have two sub-problems.

1) Axial sub-problem (Problem I): Homogeneous partial differential equation (PDE) with nonhomogeneous boundary conditions, $u_{1}^{(m)}$.

2) Axial sub-problem (Problem II): Nonhomogeneous partial differential equation (PDE) with homogeneous boundary conditions, $u_{2}^{(m)}$.

$$
u^{(m)}=u_{1}^{(m)}+u_{2}^{(m)}
$$

$\underline{\text { Axial sub-problem (Problem I) for axial stress distribution for } u_{1}^{(m)}}$

$$
\begin{gathered}
\frac{\partial^{2} u_{1}^{(m)}}{\partial x^{2}}-\frac{1}{\left(c_{e l}^{(m)}\right)^{2}} \frac{\partial^{2} u_{1}^{(m)}}{\partial t^{2}}=0 \\
\mathrm{BC}: \quad u_{1}^{(m)}(0, t)=V t, \quad u_{1}^{(m)}(L, t)=0 \\
\mathrm{IC}: \quad u_{1}^{(m)}(x, 0)=0, \quad \frac{\partial u_{1}^{(m)}}{\partial t}(L, t)=0
\end{gathered}
$$

Dividing $\mathrm{u}_{1}$ into a reference solution $u_{1}^{(\text {ref })}$ and a perturbation solution $v_{1}$ [80].

$$
u_{1}=u_{1}^{(r e f)}(x, t)+v_{1}(x, t)
$$

$u_{1}^{(r e f)}(x, t)$ can be obtained by neglecting the second term in LHS of (2.24). 


$$
u_{h}^{(r e f)}(x)=\frac{V t x}{L}
$$

From separation of variables, the perturbation solution $v_{1}(x, t)$ is given by

$$
\begin{aligned}
& v_{1}(x, t)=X(x) \cdot T(t) \\
& \frac{X^{\prime \prime}}{X}=\frac{1}{c_{0}^{2}} \frac{T^{\prime \prime}}{T}=-\lambda \\
& X=c_{1} \cos \sqrt{\lambda} x+c_{2} \sin \sqrt{\lambda} x
\end{aligned}
$$

To apply the homogeneous boundary conditions in the perturbation problem of "Problem 1", the eigenfunction is

$$
\begin{gathered}
X \sim \sin \frac{n \pi x}{L} \\
v_{h}(x, t)=\sum_{n=1}^{\infty} A_{n} \sin \left(\frac{n \pi x}{L}\right) \cdot \sin \left(\frac{c_{0} n \pi t}{L}\right)
\end{gathered}
$$

Applying the initial conditions, Eqn. (2.25c,d) to Eqns. (A1), (A2), and (A7),

$$
\begin{aligned}
& A_{n}=-\frac{2}{c_{e l}^{(m)} n \pi} \int_{0}^{L} \frac{V x}{L} \sin \left(\frac{n \pi x}{L}\right) \cdot d x=\frac{2 L V}{c_{e l}^{(m)} n^{2} \pi^{2}}(-1)^{n} \\
& u_{1}=\frac{V x t}{L}+\frac{2 V L}{c_{e l}^{(m)} \pi^{2}} \sum_{n=1}^{\infty} \frac{(-1)^{n}}{n^{2}} \sin \left(\frac{n \pi x}{L}\right) \sin \left(\frac{c_{e l}^{(m)} n \pi t}{L}\right)
\end{aligned}
$$

$\underline{\text { Axial sub-problem (Problem II) for axial stress distribution for } u_{2}^{(m)}}$

$$
\begin{gathered}
\frac{\partial^{2} u_{2}^{(m)}}{\partial x^{2}}-\frac{1}{\left(c_{e l}^{(m)}\right)^{2}} \frac{\partial^{2} u_{2}^{(m)}}{\partial t^{2}}=\frac{1}{2} \frac{\partial}{\partial x}\left[\left(\frac{\partial\left(w^{(m)}+w_{0}^{(m)}\right)}{\partial x}\right)^{2}-\left(\frac{\partial w_{0}^{(m)}}{\partial x}\right)^{2}\right] \\
\mathrm{BC}: \quad u_{2}^{(m)}(0, t)=0, \quad u_{2}^{(m)}(L, t)=0
\end{gathered}
$$




$$
\mathrm{IC}: \quad u_{2}^{(m)}(x, 0)=0, \quad \frac{\partial u_{2}^{(m)}}{\partial t}(L, t)=0
$$

To solve "Problem 2", the related eigen-problem can be written as below [34].

\section{$<$ Related Eigenproblem $>$}

$$
\begin{aligned}
u_{2}^{(m)}=\mathrm{X}(\mathrm{x}) \mathrm{T}(\mathrm{t}) \\
\quad X^{\prime \prime}+\lambda X=0 \\
\quad X=C_{1} \cdot \cos \sqrt{\lambda} x+C_{2} \cdot \sin \sqrt{\lambda} x
\end{aligned}
$$

From the boundary conditions Eqns. (2.27a,b),

$$
\mathrm{C}_{1}=0 \text {, and } \sqrt{\lambda} L=n \pi \text { for a nontrivial solution, and thus, }
$$

$$
X=\sin \frac{n \pi x}{L}
$$

Therefore, the solution, $u_{2}^{(m)}$, can be approximated as below.

$$
u_{2}^{(m)}(x, t) \sim \sum a_{n}(t) \sin \frac{n \pi x}{L}
$$

Substituting (A13) into Eqn. (2.26) to get $\mathrm{a}_{\mathrm{n}}(\mathrm{t})$,

$$
\frac{1}{\left(c_{e l}^{(m)}\right)^{2}} \ddot{a}_{n} \cdot \sin \frac{n \pi x}{L}+a_{n}\left(\frac{n \pi}{L}\right)^{2} \cdot \sin \frac{n \pi x}{L}=-\frac{1}{2} \frac{\partial}{\partial x}\left[\left(\frac{\partial\left(w+w_{o}\right)}{\partial x}\right)^{2}-\left(\frac{\partial w_{o}}{\partial x}\right)^{2}\right]
$$

Multiplying (A.22) by $X(x)$ and integrating over the range [0,L], Eqn. (A14) becomes

$$
\ddot{a}_{n}+\left(\frac{n \pi}{L}\right)^{2}\left(c_{e l}^{(m)}\right)^{2} \cdot a_{n}=-\frac{\left(c_{e l}^{(m)}\right)^{2}}{(L / 2)} \int_{0}^{L} \frac{1}{2} \frac{\partial}{\partial x}\left[\left(\frac{\partial\left(w+w_{o}\right)}{\partial x}\right)^{2}-\left(\frac{\partial w_{o}}{\partial x}\right)^{2}\right] \cdot \sin \frac{n \pi x}{L} d x
$$

Integrating by parts, the RHS of Eqn. (A15) can be modified as below 


$$
\ddot{a}_{n}+\left(\frac{n \pi}{L}\right)^{2}\left(c_{e l}^{(m)}\right)^{2} \cdot a_{n}=\left(\frac{\left(c_{e l}^{(m)}\right)^{2} n \pi}{L^{2}}\right) \cdot \int_{0}^{L}\left[\left(\frac{\partial\left(w+w_{o}\right)}{\partial x}\right)^{2}-\left(\frac{\partial w_{o}}{\partial x}\right)^{2}\right] \cdot \cos \frac{n \pi x}{L} d x
$$

To solve $\mathrm{a}_{\mathrm{n}}(\mathrm{t})$ in the ODE (A16), the solution can be divided into homogeneous and particular solutions.

$$
a_{n}^{\text {homogeneous }}(t)=C_{3} \cos \frac{c_{e l}^{(m)} n \pi}{L} t+C_{4} \sin \frac{c_{e l}^{(m)} n \pi}{L} t
$$

To get $a_{n}^{\text {particular }}(t)$, Variation of Parameters is applied.

From the homogeneous solutions, $a_{n}^{\text {homogeneous }}(t)$, the Wronskian, W, is

$$
\begin{aligned}
& W=\left|\begin{array}{cc}
u_{1} & u_{2} \\
u_{1, t} & u_{2, t}
\end{array}\right|=\left|\begin{array}{cc}
\cos \frac{c_{e l}^{(m)} n \pi}{L} t & \sin \frac{c_{e l}^{(m)} n \pi}{L} t \\
-\frac{c_{e l}^{(m)} n \pi}{L} \sin \frac{c_{e l}^{(m)} n \pi}{L} t & \frac{c_{e l}^{(m)} n \pi}{L} \cos \frac{c_{e l}^{(m)} n \pi}{L} t
\end{array}\right|=\frac{c_{e l}^{(m)} n \pi}{L} \\
& V_{1, t}=-\frac{f}{W} u_{2}=-\frac{\left(\frac{\left(c_{e l}^{(m)}\right)^{2} n \pi}{L^{2}}\right) \cdot \int_{0}^{L}\left[\left(\frac{\partial\left(w+w_{o}\right)}{\partial x}\right)^{2}-\left(\frac{\partial w_{o}}{\partial x}\right)^{2}\right] \cdot \cos \frac{n \pi x}{L} d x}{\left(\frac{c_{e l}^{(m)} n \pi}{L}\right)} \sin \frac{c_{e l}^{(m)} n \pi}{L} t \\
& V_{2, t}=\frac{f}{W} u_{1}=\frac{\left(\frac{\left(c_{e l}^{(m)}\right)^{2} n \pi}{L^{2}}\right) \cdot \int_{0}^{L}\left[\left(\frac{\partial\left(w+w_{o}\right)}{\partial x}\right)^{2}-\left(\frac{\partial w_{o}}{\partial x}\right)^{2}\right] \cdot \cos \frac{n \pi x}{L} d x}{\left(\frac{c_{e l}^{(m)} n \pi}{L}\right)} \cos \frac{c_{e l}^{(m)} n \pi}{L} t \\
& V_{1}=\int_{0}^{t} V_{1, \tau} d \tau=-\frac{c_{e l}^{(m)}}{L} \int_{0}^{t}\left[\int_{0}^{L}\left[\left(\frac{\partial\left(w+w_{o}\right)}{\partial x}\right)^{2}-\left(\frac{\partial w_{o}}{\partial x}\right)^{2}\right] \cdot \cos \frac{n \pi x}{L} d x\right] \cdot \sin \frac{c_{e l}^{(m)} n \pi \tau}{L} d \tau \\
& V_{2}=\int_{0}^{t} V_{2, \tau} d \tau=\frac{c_{e l}^{(m)}}{L} \int_{0}^{t}\left[\int_{0}^{L}\left[\left(\frac{\partial\left(w+w_{o}\right)}{\partial x}\right)^{2}-\left(\frac{\partial w_{o}}{\partial x}\right)^{2}\right] \cdot \cos \frac{n \pi x}{L} d x\right] \cdot \cos \frac{c_{e l}^{(m)} n \pi \tau}{L} d \tau
\end{aligned}
$$

Therefore, 


$$
\begin{aligned}
a_{n}(t)= & a_{n}^{\text {hom }}(t)+a_{n}^{\text {part }}(t) \\
= & C_{3} \cos \frac{c_{e l}^{(m)} n \pi}{L} t+C_{4} \sin \frac{c_{e l}^{(m)} n \pi}{L} t+ \\
& -\frac{c_{e l}^{(m)}}{L} \int_{0}^{t}\left[\int_{0}^{L}\left[\left(\frac{\partial\left(w+w_{o}\right)}{\partial x}\right)^{2}-\left(\frac{\partial w_{o}}{\partial x}\right)^{2}\right] \cdot \cos \frac{n \pi x}{L} d x\right] \cdot \sin \frac{c_{e l}^{(m)} n \pi \tau}{L} d \tau \cdot \cos \frac{c_{e l}^{(m)} n \pi}{L} t \\
& +\frac{c_{e l}^{(m)}}{L} \int_{0}^{t}\left[\int_{0}^{L}\left[\left(\frac{\partial\left(w+w_{o}\right)}{\partial x}\right)^{2}-\left(\frac{\partial w_{o}}{\partial x}\right)^{2}\right] \cdot \cos \frac{n \pi x}{L} d x\right] \cdot \cos \frac{c_{e l}^{(m)} n \pi \tau}{L} d \tau \cdot \sin \frac{c_{e l}^{(m)} n \pi}{L} t
\end{aligned}
$$

Note that $\mathrm{w}$ is a function of $\tau$ and $\mathrm{x}$ in Eqn. (A20a).

$$
\begin{aligned}
& a_{n}(t)=C_{3} \cos \frac{c_{e l}^{(m)} n \pi}{L} t+C_{4} \sin \frac{c_{e l}^{(m)} n \pi}{L} t+ \\
&+\frac{c_{e l}^{(m)}}{L} \int_{0}^{t}\left[\int_{0}^{L}\left[\left(\frac{\partial\left(w+w_{o}\right)}{\partial x}\right)^{2}-\left(\frac{\partial w_{o}}{\partial x}\right)^{2}\right] \cdot \cos \frac{n \pi x}{L} d x\right] \cdot \sin \frac{c_{e l}^{(m)} n \pi(t-\tau)}{L} d \tau
\end{aligned}
$$

Substituting the ICs, Eqns (2.27c,d) into (A20b), the coefficient $a_{n}(t)$ can be attained as follows.

$$
\therefore a_{n}(t)=\frac{c_{e l}^{(m)}}{L} \int_{0}^{t}\left[\int_{0}^{L}\left[\left(\frac{\partial\left(w+w_{o}\right)}{\partial x}\right)^{2}-\left(\frac{\partial w_{o}}{\partial x}\right)^{2}\right] \cdot \cos \frac{n \pi x}{L} d x\right] \cdot \sin \frac{c_{e l}^{(m)} n \pi(t-\tau)}{L} d \tau
$$

Rewriting (A13) with (A21),

$$
\begin{aligned}
& u_{P}(x, t) \sim \sum a_{n}(t) \sin \frac{n \pi x}{L} \\
& =\sum_{n=1}^{\infty}\left\{\frac{c_{e l}^{(m)}}{L} \int_{0}^{t}\left[\int_{0}^{L}\left[\left(\frac{\partial\left(w+w_{o}\right)}{\partial x}\right)^{2}-\left(\frac{\partial w_{o}}{\partial x}\right)^{2}\right] \cdot \cos \frac{n \pi x}{L} d x\right] \cdot \sin \frac{c_{e l}^{(m)} n \pi(t-\tau)}{L} d \tau\right\} \cdot \sin \frac{n \pi x}{L}
\end{aligned}
$$




\section{APPENDIX B APPROXIMATION OF a(X) IN SECTION 3.3.5}

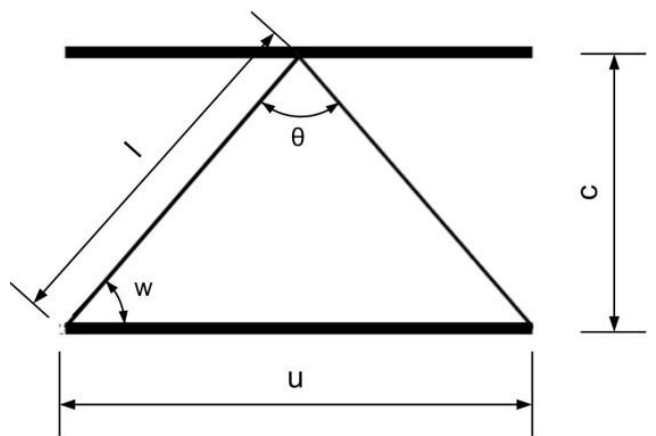

(a)

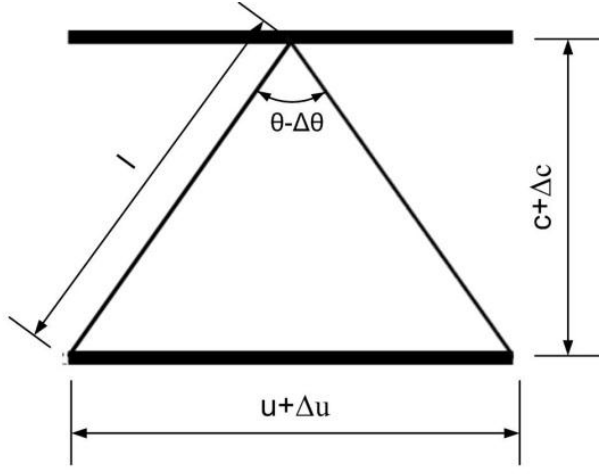

(b)

Figure B1: Change of vertical position of a node due to in-plane loading: (a) Before in-plane compression; (b) After in-plane compression.

As shown in Fig. B1, the change of core height assuming a corrugation leg as a rigid link,

$$
\Delta c=\ell \cos \left(\frac{\theta}{2}\right)-\ell \cos \left(\frac{\theta-\Delta \theta}{2}\right)
$$

For small $\Delta \theta$, Eqn. (B1) is reduced to

$$
\Delta c \approx \ell\left\{\sin \left(\frac{\theta}{2}\right) \sin \left(\frac{\Delta \theta}{2}\right)\right\}
$$

The width change of the unit-cell, $\Delta \mathrm{u}$, is given as below.

$$
\Delta u \approx 2 \ell\left\{\cos \left(\frac{\theta}{2}\right) \sin \left(\frac{\Delta \theta}{2}\right)\right\}
$$

From Eqns. (B2) and (B3), the kinematic relation between $\Delta \mathrm{c}$ and $\Delta \mathrm{u}$ is

$$
\Delta c=\left(\frac{\Delta u}{2}\right) \tan \left(\frac{\pi}{2}-\omega\right)
$$

Since shortening of the face sheet is involved in the width change of the unit-cell, $\Delta \mathrm{u}$, 


$$
\Delta u \approx\left(\frac{\Delta \sigma_{e l}^{(P e r p)}}{E /\left(1-v^{2}\right)}\right)[2 \ell \cos (\varpi)]
$$

Substituting (B5) into (B4),

$$
\therefore \frac{\Delta c}{2} \approx \frac{1}{2} \cdot\left(\frac{\Delta \sigma_{e l}^{(P e r p)}}{E /\left(1-v^{2}\right)} \ell \cos (\omega)\right) \tan \left(\frac{\pi}{2}-\omega\right)
$$

Therefore, $\Delta c / 2$ in Eqn. (B6) is employed as the value of $\mathrm{a}_{1}$ in Eqn. (3.13). 


\section{APPENDIX C DETERMINATION OF THE PATCH SIZE}

Consider the size of patch on elastic plates, $a \times b$. One of dimensions, $b$, is determined by a geometric dimension: i.e. $\mathrm{b}=2 \ell \cdot \cos \omega$ for the analysis of face plates, and $\mathrm{b}=\ell$ for core plates, respectively. On the other hand, the other dimension ' $\mathrm{a}$ ' is assumed to be such that $\mathrm{N}_{\mathrm{x}}$ is minimized in the eigen problem.

Eqn. (4.1) in the absence of $\mathrm{w}_{0}$ and the inertia term is given by:

$$
D\left(\frac{\partial^{4} w}{\partial x^{4}}+2 \frac{\partial^{4} w}{\partial x^{2} \partial y^{2}}+\frac{\partial^{4} w}{\partial y^{4}}\right)+N_{x} \frac{\partial^{2} w}{\partial x^{2}}+N_{y} \frac{\partial^{2} w}{\partial y^{2}}=0
$$

Applying Ritz method in Eqn. (C1) with the relationship of $\mathrm{N}_{\mathrm{y}}=\mathrm{vN}_{\mathrm{x}}$ and the assumed displacement in Eqn (4.4), Eqn. (C1) becomes Eqn. (C2).

$$
\left[\int_{A} w_{, x x} \cdot w_{, x x} d A+2 \int_{A} w_{, x y} \cdot w_{, x y} d A+\int_{A} w_{, y y} \cdot w_{, y y} d A\right]-\frac{N_{x}}{D} \int_{A} w_{, x} \cdot w_{, x} d A-\frac{N_{y}}{D} \int_{A} w_{, y} \cdot w_{, y} d A=0
$$

Therefore, $\mathrm{N}_{\mathrm{x}}$ is given in terms of $\mathrm{a}$ and $\mathrm{b}$.

$$
\begin{aligned}
N_{x} & =\frac{D \pi^{2}\left(\frac{3}{4}\left(\frac{1}{a^{4}}\right)+2\left(\frac{1}{a^{2} b^{2}}\right)+\left(\frac{4}{b^{4}}\right)\right)}{\left\{\frac{3}{4}\left(\frac{1}{a^{2}}\right)+v\left(\frac{1}{b^{2}}\right)\right\}} \\
& =\frac{D \pi^{2}}{b^{2}} \cdot \frac{\frac{3}{4}+2\left(\frac{a}{b}\right)^{2}+4\left(\frac{a}{b}\right)^{4}}{\left(\frac{a}{b}\right)^{2} \cdot\left[\frac{3}{4}+v\left(\frac{a}{b}\right)^{2}\right]}
\end{aligned}
$$

Let $x=\frac{a}{b}$, and Eqn. (C3) is rewritten as:

$$
N_{x}=\frac{D \pi^{2}}{b^{2}} \cdot \frac{\frac{3}{4}+2 x^{2}+4 x^{4}}{x^{2} \cdot\left[\frac{3}{4}+v x^{2}\right]}
$$


To find ' $x$ ' for minimizing $\mathrm{N}_{\mathrm{x}}$ with the fixed $\mathrm{b}$, the following equation (C5) should be solved.

$$
\frac{d N_{x}}{d x}=-\frac{D \pi^{2}}{b^{2}}\left(\frac{\left(4 x+16 x^{3}\right)\left(v x^{4}+\frac{3}{4} x^{2}\right)-\left(\frac{3}{4}+2 x^{2}+4 x^{4}\right)\left(4 v x^{3}+\frac{3}{2} x\right)}{\left(v x^{4}+\frac{3}{4} x^{2}\right)^{2}}\right)=0
$$

For v=0.33 in case of Al6061-T6,

$$
\begin{aligned}
& x=\left(\frac{a}{b}\right)=0.779319, \\
& N_{x, \min }=5.9597 \frac{D \pi^{2}}{b^{2}}
\end{aligned}
$$

For $\mathrm{v}=0.3$ in case of $\mathrm{SS} 304$,

$$
\begin{array}{r}
x=\left(\frac{a}{b}\right)=0.766072, \\
N_{x}=6.0746 \frac{D \pi^{2}}{b^{2}}
\end{array}
$$

If assuming no constraint on the lateral expansion $\mathrm{v}=0$ (i.e., $\mathrm{N}_{\mathrm{y}}=0$ )

$$
\begin{aligned}
x=\left(\frac{a}{b}\right) & =\frac{\sqrt[4]{3}}{2}=0.658037 \\
N_{x, \min } & =7.28547 \frac{D \pi^{2}}{b^{2}}
\end{aligned}
$$

Compare $\operatorname{Eqn}(\mathrm{C} 8 \mathrm{~b})$ with the exact value of $N_{x}^{(\text {minimized })} \approx 6.97 \frac{D \pi^{2}}{b^{2}}$ in uniaxial case[100].

The eigenproblem of inelastic plates from Becque's equation is given by 


$$
E_{t} I \varphi \frac{\partial^{4} w}{\partial x^{4}}+F I \varphi \frac{\partial^{4} w}{\partial x^{2} \partial y^{2}}+E_{0} I \varphi \frac{\partial^{4} w}{\partial y^{4}}+N_{x} \frac{\partial^{2} w}{\partial x^{2}}+N_{y} \frac{\partial^{2} w}{\partial y^{2}}=0
$$

And then, Eqn. (C9) is transformed into (C10) likewise in the elastic analysis.

$$
E_{t} I \varphi \int w_{, x x} w_{, x x} d A+F I \varphi \int w_{, x y} w_{, x y} d A+E_{0} I \varphi \int w_{, y y} w_{, y y} d A-N_{x} \int w_{, x} w_{, x} d A-N_{y} \int w_{, y} w_{, y} d A=0
$$

From Eqn. (C10), $\mathrm{N}_{\mathrm{x}}$ is given using the relationship of $\mathrm{N}_{\mathrm{y}}=\mathrm{v}_{\mathrm{p}} \mathrm{N}_{\mathrm{x}}$, and the assumed displacement described in Eqns. (4.4).

$$
N_{x}=\frac{I \varphi \pi^{2}}{b^{2}} \frac{\frac{3}{4} E_{t}+2 F\left(\frac{a}{b}\right)^{2}+E_{0} \cdot 4\left(\frac{a}{b}\right)^{4}}{\left(\frac{a}{b}\right)^{2} \cdot\left(\frac{3}{4}+v_{P}\left(\frac{a}{b}\right)^{2}\right)}
$$

Let $\mathrm{x}=\left(\frac{a}{b}\right)$, and then $(\mathrm{C} 11 \mathrm{a})$ is written as

$$
N_{x}=\frac{I \varphi \pi^{2}}{b^{2}} \frac{\frac{3}{4} E_{t}+2 F x^{2}+4 E_{0} x^{4}}{x^{2} \cdot\left(\frac{3}{4}+v_{P} x^{2}\right)}
$$

To find ' $x$ ' for minimizing $\mathrm{N}_{\mathrm{x}}$ with the fixed $\mathrm{b}$, $\mathrm{x}$ should be determined such that $\frac{d N_{x}}{d x}=0 \mathrm{using}(\mathrm{C} 11 \mathrm{~b})$

For the material properties of Al6061,

$$
\mathrm{x}=\left(\frac{a}{b}\right)=0.193916
$$


For the material properties of the rate-independent SS304,

$$
\mathrm{x}=\left(\frac{a}{b}\right)=0.290168
$$

If assuming no restraint on the lateral expansion,

$$
x=\left(\frac{a}{b}\right)=\sqrt[4]{\frac{E_{t}}{E_{0}}} \frac{\sqrt[4]{3}}{2}
$$

Compare Eqns. (C12a), (C12b), (C12c) with Eqns. (C6a), (C7a), (C8a). The patch size in inelastic plates is less than one half the sizes in elastic plates. It is a reasonable derivation in that elastic-plastic plates buckle with shorter wave lengths than those of elastic plates. 


\section{APPENDIX D FULL FACTORIAL EXPERIMENTAL DESIGN}

Table D-1. Full Factorial experimental design for global buckling response of sandwich columns (Perpendicular-to-corrugations) under $\mathrm{V}=0.1 \mathrm{~m} / \mathrm{s}$ and $\mathrm{V}=1 \mathrm{~m} / \mathrm{s}$.

\begin{tabular}{|c|c|c|c|c|c|c|c|c|c|c|c|c|c|c|}
\hline $\begin{array}{l}\text { Exp. } \\
\text { No.. }\end{array}$ & $\mathrm{h}$ & $\mathrm{t}$ & 1 & $\mathrm{w}$ & $\begin{array}{c}\text { Exp. } \\
\text { No. }\end{array}$ & $\mathrm{h}$ & $\mathrm{t}$ & 1 & $\mathrm{w}$ & $\begin{array}{l}\text { Exp. } \\
\text { No. }\end{array}$ & $\mathrm{h}$ & $\mathrm{t}$ & 1 & $\mathrm{w}$ \\
\hline 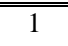 & 0.5 & 0.5 & $\bar{~} 4$ & 30 & 39 & 0.5 & 1 & 250 & 30 & 77 & 0.5 & 2 & 30 & 45 \\
\hline 2 & 0.5 & 0.5 & 8 & 30 & 40 & 0.5 & 1 & 60 & 30 & 78 & 0.5 & 2 & 40 & 45 \\
\hline 3 & 0.5 & 0.5 & 12 & 30 & 41 & 0.5 & 1 & 4 & 45 & 79 & 0.5 & 2 & 50 & 45 \\
\hline 4 & 0.5 & 0.5 & 16 & 30 & 42 & 0.5 & 1 & 8 & 45 & 80 & 0.5 & 2 & 60 & 45 \\
\hline 5 & 0.5 & 0.5 & 20 & 30 & 43 & 0.5 & 1 & 12 & 45 & 81 & 0.5 & 2 & 4 & 60 \\
\hline 6 & 0.5 & 0.5 & 25 & 30 & 44 & 0.5 & 1 & 16 & 45 & 82 & 0.5 & 2 & 8 & 60 \\
\hline 7 & 0.5 & 0.5 & 30 & 30 & 45 & 0.5 & 1 & 20 & 45 & 83 & 0.5 & 2 & 12 & 60 \\
\hline 8 & 0.5 & 0.5 & 40 & 30 & 46 & 0.5 & 1 & 25 & 45 & 84 & 0.5 & 2 & 16 & 60 \\
\hline 9 & 0.5 & 0.5 & 50 & 30 & 47 & 0.5 & 1 & 30 & 45 & 85 & 0.5 & 2 & 20 & 60 \\
\hline 10 & 0.5 & 0.5 & 60 & 30 & 48 & 0.5 & 1 & 40 & 45 & 86 & 0.5 & 2 & 25 & 60 \\
\hline 11 & 0.5 & 0.5 & 4 & 45 & 49 & 0.5 & 1 & 50 & 45 & 87 & 0.5 & 2 & 30 & 60 \\
\hline 12 & 0.5 & 0.5 & 8 & 45 & 50 & 0.5 & 1 & 60 & 45 & 88 & 0.5 & 2 & 40 & 60 \\
\hline 13 & 0.5 & 0.5 & 12 & 45 & 51 & 0.5 & 1 & 4 & 60 & 89 & 0.5 & 2 & 50 & 60 \\
\hline 14 & 0.5 & 0.5 & 16 & 45 & 52 & 0.5 & 1 & 8 & 60 & 90 & 0.5 & 2 & 60 & 60 \\
\hline 15 & 0.5 & 0.5 & 20 & 45 & 53 & 0.5 & 1 & 12 & 60 & 91 & 1 & 0.5 & 4 & 30 \\
\hline 16 & 0.5 & 0.5 & 25 & 45 & 54 & 0.5 & 1 & 16 & 60 & 92 & 1 & 0.5 & 8 & 30 \\
\hline 17 & 0.5 & 0.5 & 30 & 45 & 55 & 0.5 & 1 & 20 & 60 & 93 & 1 & 0.5 & 12 & 30 \\
\hline 18 & 0.5 & 0.5 & 40 & 45 & 56 & 0.5 & 1 & 25 & 60 & 94 & 1 & 0.5 & 16 & 30 \\
\hline 19 & 0.5 & 0.5 & 50 & 45 & 57 & 0.5 & 1 & 30 & 60 & 95 & 1 & 0.5 & 20 & 30 \\
\hline 20 & 0.5 & 0.5 & 60 & 45 & 58 & 0.5 & 1 & 40 & 60 & 96 & 1 & 0.5 & 25 & 30 \\
\hline 21 & 0.5 & 0.5 & 4 & 60 & 59 & 0.5 & 1 & 50 & 60 & 97 & 1 & 0.5 & 30 & 30 \\
\hline 22 & 0.5 & 0.5 & 8 & 60 & 60 & 0.5 & 1 & 60 & 60 & 98 & 1 & 0.5 & 40 & 30 \\
\hline 23 & 0.5 & 0.5 & 12 & 60 & 61 & 0.5 & 2 & 4 & 30 & 99 & 1 & 0.5 & 50 & 30 \\
\hline 24 & 0.5 & 0.5 & 16 & 60 & 62 & 0.5 & 2 & 8 & 30 & 100 & 1 & 0.5 & 60 & 30 \\
\hline 25 & 0.5 & 0.5 & 20 & 60 & 63 & 0.5 & 2 & 12 & 30 & 101 & 1 & 0.5 & 4 & 45 \\
\hline 26 & 0.5 & 0.5 & 25 & 60 & 64 & 0.5 & 2 & 16 & 30 & 102 & 1 & 0.5 & 8 & 45 \\
\hline 27 & 0.5 & 0.5 & 30 & 60 & 65 & 0.5 & 2 & 20 & 30 & 103 & 1 & 0.5 & 12 & 45 \\
\hline 28 & 0.5 & 0.5 & 40 & 60 & 66 & 0.5 & 2 & 25 & 30 & 104 & 1 & 0.5 & 16 & 45 \\
\hline 29 & 0.5 & 0.5 & 50 & 60 & 67 & 0.5 & 2 & 30 & 30 & 105 & 1 & 0.5 & 20 & 45 \\
\hline 30 & 0.5 & 0.5 & 60 & 60 & 68 & 0.5 & 2 & 40 & 30 & 106 & 1 & 0.5 & 25 & 45 \\
\hline 31 & 0.5 & 1 & 4 & 30 & 69 & 0.5 & 2 & 50 & 30 & 107 & 1 & 0.5 & 30 & 45 \\
\hline 32 & 0.5 & 1 & 8 & 30 & 70 & 0.5 & 2 & 60 & 30 & 108 & 1 & 0.5 & 40 & 45 \\
\hline 33 & 0.5 & 1 & 12 & 30 & 71 & 0.5 & 2 & 4 & 45 & 109 & 1 & 0.5 & 50 & 45 \\
\hline 34 & 0.5 & 1 & 16 & 30 & 72 & 0.5 & 2 & 8 & 45 & 110 & 1 & 0.5 & 60 & 45 \\
\hline 35 & 0.5 & 1 & 20 & 30 & 73 & 0.5 & 2 & 12 & 45 & 111 & 1 & 0.5 & 4 & 60 \\
\hline 36 & 0.5 & 1 & 25 & 30 & 74 & 0.5 & 2 & 16 & 45 & 112 & 1 & 0.5 & 8 & 60 \\
\hline 37 & 0.5 & 1 & 30 & 30 & 75 & 0.5 & 2 & 20 & 45 & 113 & 1 & 0.5 & 12 & 60 \\
\hline 38 & 0.5 & 1 & 40 & 30 & 76 & 0.5 & 2 & 25 & 45 & 114 & 1 & 0.5 & 16 & 60 \\
\hline
\end{tabular}


Table D-1. Full Factorial experimental design for global buckling response of sandwich columns (Perpendicular-to-corrugations) under $\mathrm{V}=0.1 \mathrm{~m} / \mathrm{s}$ and $\mathrm{V}=1 \mathrm{~m} / \mathrm{s}$ (Continued).

\begin{tabular}{|c|c|c|c|c|c|c|c|c|c|c|c|c|c|c|}
\hline $\begin{array}{l}\text { Exp. } \\
\text { No. }\end{array}$ & $\mathrm{h}$ & $\mathrm{t}$ & 1 & W & $\begin{array}{l}\text { Exp. } \\
\text { No. }\end{array}$ & $\mathrm{h}$ & $\mathrm{t}$ & 1 & W & $\begin{array}{l}\text { Exp. } \\
\text { No.. }\end{array}$ & $\mathrm{h}$ & $\mathrm{t}$ & 1 & $\mathrm{w}$ \\
\hline 1115 & $\bar{~} 1$ & 0.5 & 20 & 60 & 156 & $\overline{1}$ & 2 & 25 & 30 & $\begin{array}{l}197 \\
\end{array}$ & 2 & 0.5 & 30 & $\overline{445}$ \\
\hline 116 & 1 & 0.5 & 25 & 60 & 157 & 1 & 2 & 30 & 30 & 198 & 2 & 0.5 & 40 & 45 \\
\hline 117 & 1 & 0.5 & 30 & 60 & 158 & 1 & 2 & 40 & 30 & 199 & 2 & 0.5 & 50 & 45 \\
\hline 118 & 1 & 0.5 & 40 & 60 & 159 & 1 & 2 & 50 & 30 & 200 & 2 & 0.5 & 60 & 45 \\
\hline 119 & 1 & 0.5 & 50 & 60 & 160 & 1 & 2 & 60 & 30 & 201 & 2 & 0.5 & 4 & 60 \\
\hline 120 & 1 & 0.5 & 60 & 60 & 161 & 1 & 2 & 4 & 45 & 202 & 2 & 0.5 & 8 & 60 \\
\hline 121 & 1 & 1 & 4 & 30 & 162 & 1 & 2 & 8 & 45 & 203 & 2 & 0.5 & 12 & 60 \\
\hline 122 & 1 & 1 & 8 & 30 & 163 & 1 & 2 & 12 & 45 & 204 & 2 & 0.5 & 16 & 60 \\
\hline 123 & 1 & 1 & 12 & 30 & 164 & 1 & 2 & 16 & 45 & 205 & 2 & 0.5 & 20 & 60 \\
\hline 124 & 1 & 1 & 16 & 30 & 165 & 1 & 2 & 20 & 45 & 206 & 2 & 0.5 & 25 & 60 \\
\hline 125 & 1 & 1 & 20 & 30 & 166 & 1 & 2 & 25 & 45 & 207 & 2 & 0.5 & 30 & 60 \\
\hline 126 & 1 & 1 & 25 & 30 & 167 & 1 & 2 & 30 & 45 & 208 & 2 & 0.5 & 40 & 60 \\
\hline 127 & 1 & 1 & 30 & 30 & 168 & 1 & 2 & 40 & 45 & 209 & 2 & 0.5 & 50 & 60 \\
\hline 128 & 1 & 1 & 40 & 30 & 169 & 1 & 2 & 50 & 45 & 210 & 2 & 0.5 & 60 & 60 \\
\hline 129 & 1 & 1 & 50 & 30 & 170 & 1 & 2 & 60 & 45 & 211 & 2 & 1 & 4 & 30 \\
\hline 130 & 1 & 1 & 60 & 30 & 171 & 1 & 2 & 4 & 60 & 212 & 2 & 1 & 8 & 30 \\
\hline 131 & 1 & 1 & 4 & 45 & 172 & 1 & 2 & 8 & 60 & 213 & 2 & 1 & 12 & 30 \\
\hline 132 & 1 & 1 & 8 & 45 & 173 & 1 & 2 & 12 & 60 & 214 & 2 & 1 & 16 & 30 \\
\hline 133 & 1 & 1 & 12 & 45 & 174 & 1 & 2 & 16 & 60 & 215 & 2 & 1 & 20 & 30 \\
\hline 134 & 1 & 1 & 16 & 45 & 175 & 1 & 2 & 20 & 60 & 216 & 2 & 1 & 25 & 30 \\
\hline 135 & 1 & 1 & 20 & 45 & 176 & 1 & 2 & 25 & 60 & 217 & 2 & 1 & 30 & 30 \\
\hline 136 & 1 & 1 & 25 & 45 & 177 & 1 & 2 & 30 & 60 & 218 & 2 & 1 & 40 & 30 \\
\hline 137 & 1 & 1 & 30 & 45 & 178 & 1 & 2 & 40 & 60 & 219 & 2 & 1 & 50 & 30 \\
\hline 138 & 1 & 1 & 40 & 45 & 179 & 1 & 2 & 50 & 60 & 220 & 2 & 1 & 60 & 30 \\
\hline 137 & 1 & 1 & 50 & 45 & 180 & 1 & 2 & 60 & 60 & 221 & 2 & 1 & 4 & 45 \\
\hline 140 & 1 & 1 & 60 & 45 & 181 & 2 & 0.5 & 4 & 30 & 222 & 2 & 1 & 8 & 45 \\
\hline 141 & 1 & 1 & 4 & 60 & 182 & 2 & 0.5 & 8 & 30 & 223 & 2 & 1 & 12 & 45 \\
\hline 142 & 1 & 1 & 8 & 60 & 183 & 2 & 0.5 & 12 & 30 & 224 & 2 & 1 & 16 & 45 \\
\hline 143 & 1 & 1 & 12 & 60 & 184 & 2 & 0.5 & 16 & 30 & 225 & 2 & 1 & 20 & 45 \\
\hline 144 & 1 & 1 & 16 & 60 & 185 & 2 & 0.5 & 20 & 30 & 226 & 2 & 1 & 25 & 45 \\
\hline 145 & 1 & 1 & 20 & 60 & 186 & 2 & 0.5 & 25 & 30 & 227 & 2 & 1 & 30 & 45 \\
\hline 146 & 1 & 1 & 25 & 60 & 187 & 2 & 0.5 & 30 & 30 & 228 & 2 & 1 & 40 & 45 \\
\hline 147 & 1 & 1 & 30 & 60 & 188 & 2 & 0.5 & 40 & 30 & 229 & 2 & 1 & 50 & 45 \\
\hline 148 & 1 & 1 & 40 & 60 & 189 & 2 & 0.5 & 50 & 30 & 230 & 2 & 1 & 60 & 45 \\
\hline 149 & 1 & 1 & 50 & 60 & 190 & 2 & 0.5 & 60 & 30 & 231 & 2 & 1 & 4 & 60 \\
\hline 150 & 1 & 1 & 60 & 60 & 191 & 2 & 0.5 & 4 & 45 & 232 & 2 & 1 & 8 & 60 \\
\hline 151 & 1 & 2 & 4 & 30 & 192 & 2 & 0.5 & 8 & 45 & 233 & 2 & 1 & 12 & 60 \\
\hline 152 & 1 & 2 & 8 & 30 & 193 & 2 & 0.5 & 12 & 45 & 234 & 2 & 1 & 16 & 60 \\
\hline 153 & 1 & 2 & 12 & 30 & 194 & 2 & 0.5 & 16 & 45 & 235 & 2 & 1 & 20 & 60 \\
\hline 154 & 1 & 2 & 16 & 30 & 195 & 2 & 0.5 & 20 & 45 & 236 & 2 & 1 & 25 & 60 \\
\hline 155 & 1 & 2 & 20 & 30 & 196 & 2 & 0.5 & 25 & 45 & 237 & 2 & 1 & 30 & 60 \\
\hline
\end{tabular}


Table D-1. Full Factorial experimental design for global buckling response of sandwich columns (Perpendicular-to-corrugations) under $\mathrm{V}=0.1 \mathrm{~m} / \mathrm{s}$ and $\mathrm{V}=1 \mathrm{~m} / \mathrm{s}$ (Continued).

\begin{tabular}{|c|c|c|c|c|c|c|c|c|c|c|c|c|c|c|}
\hline $\begin{array}{l}\text { Exp. } \\
\text { No. }\end{array}$ & $\mathrm{h}$ & $\mathrm{t}$ & 1 & $\mathrm{~W}$ & $\begin{array}{l}\text { Exp. } \\
\text { No. }\end{array}$ & $\mathrm{h}$ & $\mathrm{t}$ & 1 & W & $\begin{array}{l}\text { Exp. } \\
\text { No.. }\end{array}$ & $\mathrm{h}$ & $\mathrm{t}$ & 1 & $\mathrm{~W}$ \\
\hline 238 & 2 & 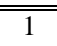 & 40 & 60 & 279 & 3 & 0.5 & 250 & 30 & 320 & 3 & (1 & 60 & $\overline{445}$ \\
\hline 239 & 2 & 1 & 50 & 60 & 280 & 3 & 0.5 & 60 & 30 & 321 & 3 & 1 & 4 & 60 \\
\hline 240 & 2 & 1 & 60 & 60 & 281 & 3 & 0.5 & 4 & 45 & 322 & 3 & 1 & 8 & 60 \\
\hline 241 & 2 & 2 & 4 & 30 & 282 & 3 & 0.5 & 8 & 45 & 323 & 3 & 1 & 12 & 60 \\
\hline 242 & 2 & 2 & 8 & 30 & 283 & 3 & 0.5 & 12 & 45 & 324 & 3 & 1 & 16 & 60 \\
\hline 243 & 2 & 2 & 12 & 30 & 284 & 3 & 0.5 & 16 & 45 & 325 & 3 & 1 & 20 & 60 \\
\hline 244 & 2 & 2 & 16 & 30 & 285 & 3 & 0.5 & 20 & 45 & 326 & 3 & 1 & 25 & 60 \\
\hline 245 & 2 & 2 & 20 & 30 & 286 & 3 & 0.5 & 25 & 45 & 327 & 3 & 1 & 30 & 60 \\
\hline 246 & 2 & 2 & 25 & 30 & 287 & 3 & 0.5 & 30 & 45 & 328 & 3 & 1 & 40 & 60 \\
\hline 247 & 2 & 2 & 30 & 30 & 288 & 3 & 0.5 & 40 & 45 & 329 & 3 & 1 & 50 & 60 \\
\hline 248 & 2 & 2 & 40 & 30 & 289 & 3 & 0.5 & 50 & 45 & 330 & 3 & 1 & 60 & 60 \\
\hline 249 & 2 & 2 & 50 & 30 & 290 & 3 & 0.5 & 60 & 45 & 331 & 3 & 2 & 4 & 30 \\
\hline 250 & 2 & 2 & 60 & 30 & 291 & 3 & 0.5 & 4 & 60 & 332 & 3 & 2 & 8 & 30 \\
\hline 251 & 2 & 2 & 4 & 45 & 292 & 3 & 0.5 & 8 & 60 & 333 & 3 & 2 & 12 & 30 \\
\hline 252 & 2 & 2 & 8 & 45 & 293 & 3 & 0.5 & 12 & 60 & 334 & 3 & 2 & 16 & 30 \\
\hline 253 & 2 & 2 & 12 & 45 & 294 & 3 & 0.5 & 16 & 60 & 335 & 3 & 2 & 20 & 30 \\
\hline 254 & 2 & 2 & 16 & 45 & 295 & 3 & 0.5 & 20 & 60 & 336 & 3 & 2 & 25 & 30 \\
\hline 255 & 2 & 2 & 20 & 45 & 296 & 3 & 0.5 & 25 & 60 & 337 & 3 & 2 & 30 & 30 \\
\hline 256 & 2 & 2 & 25 & 45 & 297 & 3 & 0.5 & 30 & 60 & 338 & 3 & 2 & 40 & 30 \\
\hline 257 & 2 & 2 & 30 & 45 & 298 & 3 & 0.5 & 40 & 60 & 339 & 3 & 2 & 50 & 30 \\
\hline 258 & 2 & 2 & 40 & 45 & 299 & 3 & 0.5 & 50 & 60 & 340 & 3 & 2 & 60 & 30 \\
\hline 259 & 2 & 2 & 50 & 45 & 300 & 3 & 0.5 & 60 & 60 & 341 & 3 & 2 & 4 & 45 \\
\hline 260 & 2 & 2 & 60 & 45 & 301 & 3 & 1 & 4 & 30 & 342 & 3 & 2 & 8 & 45 \\
\hline 261 & 2 & 2 & 4 & 60 & 302 & 3 & 1 & 8 & 30 & 343 & 3 & 2 & 12 & 45 \\
\hline 262 & 2 & 2 & 8 & 60 & 303 & 3 & 1 & 12 & 30 & 344 & 3 & 2 & 16 & 45 \\
\hline 263 & 2 & 2 & 12 & 60 & 304 & 3 & 1 & 16 & 30 & 345 & 3 & 2 & 20 & 45 \\
\hline 264 & 2 & 2 & 16 & 60 & 305 & 3 & 1 & 20 & 30 & 346 & 3 & 2 & 25 & 45 \\
\hline 265 & 2 & 2 & 20 & 60 & 306 & 3 & 1 & 25 & 30 & 347 & 3 & 2 & 30 & 45 \\
\hline 266 & 2 & 2 & 25 & 60 & 307 & 3 & 1 & 30 & 30 & 348 & 3 & 2 & 40 & 45 \\
\hline 267 & 2 & 2 & 30 & 60 & 308 & 3 & 1 & 40 & 30 & 349 & 3 & 2 & 50 & 45 \\
\hline 268 & 2 & 2 & 40 & 60 & 309 & 3 & 1 & 50 & 30 & 350 & 3 & 2 & 60 & 45 \\
\hline 267 & 2 & 2 & 50 & 60 & 310 & 3 & 1 & 60 & 30 & 351 & 3 & 2 & 4 & 60 \\
\hline 270 & 2 & 2 & 60 & 60 & 311 & 3 & 1 & 4 & 45 & 352 & 3 & 2 & 8 & 60 \\
\hline 271 & 3 & 0.5 & 4 & 30 & 312 & 3 & 1 & 8 & 45 & 353 & 3 & 2 & 12 & 60 \\
\hline 272 & 3 & 0.5 & 8 & 30 & 313 & 3 & 1 & 12 & 45 & 354 & 3 & 2 & 16 & 60 \\
\hline 273 & 3 & 0.5 & 12 & 30 & 314 & 3 & 1 & 16 & 45 & 355 & 3 & 2 & 20 & 60 \\
\hline 274 & 3 & 0.5 & 16 & 30 & 315 & 3 & 1 & 20 & 45 & 356 & 3 & 2 & 25 & 60 \\
\hline 275 & 3 & 0.5 & 20 & 30 & 316 & 3 & 1 & 25 & 45 & 357 & 3 & 2 & 30 & 60 \\
\hline 276 & 3 & 0.5 & 25 & 30 & 317 & 3 & 1 & 30 & 45 & 358 & 3 & 2 & 40 & 60 \\
\hline 277 & 3 & 0.5 & 30 & 30 & 318 & 3 & 1 & 40 & 45 & 359 & 3 & 2 & 50 & 60 \\
\hline 278 & 3 & 0.5 & 40 & 30 & 319 & 3 & 1 & 50 & 45 & 360 & 3 & 2 & 60 & 60 \\
\hline
\end{tabular}


Table D-2. Full Factorial experimental design for face wrinkling response of sandwich columns compressed perpendicular-to-corrugations under $\mathrm{V}=0.1 \mathrm{~m} / \mathrm{s}$ and $\mathrm{V}=1 \mathrm{~m} / \mathrm{s}$.

\begin{tabular}{|c|c|c|c|c|c|c|c|c|c|c|c|c|c|c|}
\hline $\begin{array}{l}\text { Exp. } \\
\text { No. }\end{array}$ & $\mathrm{h}$ & $\mathrm{t}$ & 1 & $\mathrm{w}$ & $\begin{array}{l}\text { Exp. } \\
\text { No. }\end{array}$ & $\mathrm{h}$ & $\mathrm{t}$ & 1 & $\mathrm{w}$ & $\begin{array}{l}\text { Exp. } \\
\text { No. }\end{array}$ & $\mathrm{h}$ & $\mathrm{t}$ & 1 & $\mathrm{w}$ \\
\hline$\overline{c 1}$ & 0.5 & 0.5 & $\bar{~} 5$ & 30 & 441 & 0.5 & $\overline{c 1}$ & 80 & 60 & 81 & 0.5 & 0.5 & $4 \quad 40$ & 260 \\
\hline 2 & 0.5 & 0.5 & 10 & 30 & 42 & 0.5 & 1 & 100 & 60 & 82 & 0.5 & 0.5 & 60 & 60 \\
\hline 3 & 0.5 & 0.5 & 20 & 30 & 43 & 0.5 & 2 & 5 & 30 & 83 & 0.5 & 0.5 & 80 & 60 \\
\hline 4 & 0.5 & 0.5 & 40 & 30 & 44 & 0.5 & 2 & 10 & 30 & 84 & 0.5 & 0.5 & 100 & 60 \\
\hline 5 & 0.5 & 0.5 & 60 & 30 & 45 & 0.5 & 2 & 20 & 30 & 85 & 0.5 & 0.5 & 5 & 30 \\
\hline 6 & 0.5 & 0.5 & 80 & 30 & 46 & 0.5 & 2 & 40 & 30 & 86 & 0.5 & 1 & 10 & 30 \\
\hline 7 & 0.5 & 0.5 & 100 & 30 & 47 & 0.5 & 2 & 60 & 30 & 87 & 0.5 & 1 & 20 & 30 \\
\hline 8 & 0.5 & 0.5 & 5 & 45 & 48 & 0.5 & 2 & 80 & 30 & 88 & 0.5 & 1 & 40 & 30 \\
\hline 9 & 0.5 & 0.5 & 10 & 45 & 49 & 0.5 & 2 & 100 & 30 & 89 & 0.5 & 1 & 60 & 30 \\
\hline 10 & 0.5 & 0.5 & 20 & 45 & 50 & 0.5 & 2 & 5 & 45 & 90 & 0.5 & 1 & 80 & 30 \\
\hline 11 & 0.5 & 0.5 & 40 & 45 & 51 & 0.5 & 2 & 10 & 45 & 91 & 1 & 1 & 100 & 30 \\
\hline 12 & 0.5 & 0.5 & 60 & 45 & 52 & 0.5 & 2 & 20 & 45 & 92 & 1 & 1 & 5 & 45 \\
\hline 13 & 0.5 & 0.5 & 80 & 45 & 53 & 0.5 & 2 & 40 & 45 & 93 & 1 & 1 & 10 & 45 \\
\hline 14 & 0.5 & 0.5 & 100 & 45 & 54 & 0.5 & 2 & 60 & 45 & 94 & 1 & 1 & 20 & 45 \\
\hline 15 & 0.5 & 0.5 & 5 & 60 & 55 & 0.5 & 2 & 80 & 45 & 95 & 1 & 1 & 40 & 45 \\
\hline 16 & 0.5 & 0.5 & 10 & 60 & 56 & 0.5 & 2 & 100 & 45 & 96 & 1 & 1 & 60 & 45 \\
\hline 17 & 0.5 & 0.5 & 20 & 60 & 57 & 0.5 & 2 & 5 & 60 & 97 & 1 & 1 & 80 & 45 \\
\hline 18 & 0.5 & 0.5 & 40 & 60 & 58 & 0.5 & 2 & 10 & 60 & 98 & 1 & 1 & 100 & 45 \\
\hline 19 & 0.5 & 0.5 & 60 & 60 & 59 & 0.5 & 2 & 20 & 60 & 99 & 1 & 1 & 5 & 60 \\
\hline 20 & 0.5 & 0.5 & 80 & 60 & 60 & 0.5 & 2 & 40 & 60 & 100 & 1 & 1 & 10 & 60 \\
\hline 21 & 0.5 & 0.5 & 100 & 60 & 61 & 0.5 & 2 & 60 & 60 & 101 & 1 & 1 & 20 & 60 \\
\hline 22 & 0.5 & 1 & 5 & 30 & 62 & 0.5 & 2 & 80 & 60 & 102 & 1 & 1 & 40 & 60 \\
\hline 23 & 0.5 & 1 & 10 & 30 & 63 & 0.5 & 2 & 100 & 60 & 103 & 1 & 1 & 60 & 60 \\
\hline 24 & 0.5 & 1 & 20 & 30 & 64 & 1 & 0.5 & 5 & 30 & 104 & 1 & 1 & 80 & 60 \\
\hline 25 & 0.5 & 1 & 40 & 30 & 65 & 1 & 0.5 & 10 & 30 & 105 & 1 & 1 & 100 & 60 \\
\hline 26 & 0.5 & 1 & 60 & 30 & 66 & 1 & 0.5 & 20 & 30 & 106 & 1 & 2 & 5 & 30 \\
\hline 27 & 0.5 & 1 & 80 & 30 & 67 & 1 & 0.5 & 40 & 30 & 107 & 1 & 2 & 10 & 30 \\
\hline 28 & 0.5 & 1 & 100 & 30 & 68 & 1 & 0.5 & 60 & 30 & 108 & 1 & 2 & 20 & 30 \\
\hline 29 & 0.5 & 1 & 5 & 45 & 69 & 1 & 0.5 & 80 & 30 & 109 & 1 & 2 & 40 & 30 \\
\hline 30 & 0.5 & 1 & 10 & 45 & 70 & 1 & 0.5 & 100 & 30 & 110 & 1 & 2 & 60 & 30 \\
\hline 31 & 0.5 & 1 & 20 & 45 & 71 & 1 & 0.5 & 5 & 45 & 111 & 1 & 2 & 80 & 30 \\
\hline 32 & 0.5 & 1 & 40 & 45 & 72 & 1 & 0.5 & 10 & 45 & 112 & 1 & 2 & 100 & 30 \\
\hline 33 & 0.5 & 1 & 60 & 45 & 73 & 1 & 0.5 & 20 & 45 & 113 & 1 & 2 & 5 & 45 \\
\hline 34 & 0.5 & 1 & 80 & 45 & 74 & 1 & 0.5 & 40 & 45 & 114 & 1 & 2 & 10 & 45 \\
\hline 35 & 0.5 & 1 & 100 & 45 & 75 & 1 & 0.5 & 60 & 45 & 115 & 1 & 2 & 20 & 45 \\
\hline 36 & 0.5 & 1 & 5 & 60 & 76 & 1 & 0.5 & 80 & 45 & 116 & 1 & 2 & 40 & 45 \\
\hline 37 & 0.5 & 1 & 10 & 60 & 77 & 1 & 0.5 & 100 & 45 & 117 & 1 & 2 & 60 & 45 \\
\hline 38 & 0.5 & 1 & 20 & 60 & 78 & 1 & 0.5 & 5 & 60 & 118 & 1 & 2 & 80 & 45 \\
\hline 39 & 0.5 & 1 & 40 & 60 & 79 & 1 & 0.5 & 10 & 60 & 119 & 1 & 2 & 100 & 45 \\
\hline 40 & 0.5 & 1 & 60 & 60 & 80 & 1 & 0.5 & 20 & 60 & 120 & 1 & 2 & 5 & 60 \\
\hline
\end{tabular}


Table D-2. Full Factorial experimental design for face wrinkling response of sandwich columns compressed perpendicular-to-corrugations under $\mathrm{V}=0.1 \mathrm{~m} / \mathrm{s}$ and $\mathrm{V}=1 \mathrm{~m} / \mathrm{s}$ (continued).

\begin{tabular}{|c|c|c|c|c|c|c|c|c|c|c|c|c|c|c|}
\hline $\begin{array}{l}\text { Exp. } \\
\text { No. }\end{array}$ & $\mathrm{h}$ & $\mathrm{t}$ & 1 & $\mathrm{w}$ & $\begin{array}{c}\text { Exp. } \\
\text { No. }\end{array}$ & $\mathrm{h}$ & $\mathrm{t}$ & 1 & $\mathrm{w}$ & $\begin{array}{l}\text { Exp. } \\
\text { No. }\end{array}$ & $\mathrm{h}$ & $\mathrm{t}$ & 1 & $\mathrm{w}$ \\
\hline 121 & $\bar{~} 1$ & 2 & 10 & 60 & 161 & 2 & 1 & 100 & 45 & 201 & 3 & 0.5 & 60 & 45 \\
\hline 122 & 1 & 2 & 20 & 60 & 162 & 2 & 1 & 5 & 60 & 202 & 3 & 0.5 & 80 & 45 \\
\hline 123 & 1 & 2 & 40 & 60 & 163 & 2 & 1 & 10 & 60 & 203 & 3 & 0.5 & 100 & 45 \\
\hline 124 & 1 & 2 & 60 & 60 & 164 & 2 & 1 & 20 & 60 & 204 & 3 & 0.5 & 5 & 60 \\
\hline 125 & 1 & 2 & 80 & 60 & 165 & 2 & 1 & 40 & 60 & 205 & 3 & 0.5 & 10 & 60 \\
\hline 126 & 1 & 2 & 100 & 60 & 166 & 2 & 1 & 60 & 60 & 206 & 3 & 0.5 & 20 & 60 \\
\hline 127 & 2 & 0.5 & 5 & 30 & 167 & 2 & 1 & 80 & 60 & 207 & 3 & 0.5 & 40 & 60 \\
\hline 128 & 2 & 0.5 & 10 & 30 & 168 & 2 & 1 & 100 & 60 & 208 & 3 & 0.5 & 60 & 60 \\
\hline 129 & 2 & 0.5 & 20 & 30 & 169 & 2 & 2 & 5 & 30 & 209 & 3 & 0.5 & 80 & 60 \\
\hline 130 & 2 & 0.5 & 40 & 30 & 170 & 2 & 2 & 10 & 30 & 210 & 3 & 0.5 & 100 & 60 \\
\hline 131 & 2 & 0.5 & 60 & 30 & 171 & 2 & 2 & 20 & 30 & 211 & 3 & 1 & 5 & 30 \\
\hline 132 & 2 & 0.5 & 80 & 30 & 172 & 2 & 2 & 40 & 30 & 212 & 3 & 1 & 10 & 30 \\
\hline 133 & 2 & 0.5 & 100 & 30 & 173 & 2 & 2 & 60 & 30 & 213 & 3 & 1 & 20 & 30 \\
\hline 134 & 2 & 0.5 & 5 & 45 & 174 & 2 & 2 & 80 & 30 & 214 & 3 & 1 & 40 & 30 \\
\hline 135 & 2 & 0.5 & 10 & 45 & 175 & 2 & 2 & 100 & 30 & 215 & 3 & 1 & 60 & 30 \\
\hline 136 & 2 & 0.5 & 20 & 45 & 176 & 2 & 2 & 5 & 45 & 216 & 3 & 1 & 80 & 30 \\
\hline 137 & 2 & 0.5 & 40 & 45 & 177 & 2 & 2 & 10 & 45 & 217 & 3 & 1 & 100 & 30 \\
\hline 138 & 2 & 0.5 & 60 & 45 & 178 & 2 & 2 & 20 & 45 & 218 & 3 & 1 & 5 & 45 \\
\hline 137 & 2 & 0.5 & 80 & 45 & 179 & 2 & 2 & 40 & 45 & 219 & 3 & 1 & 10 & 45 \\
\hline 140 & 2 & 0.5 & 100 & 45 & 180 & 2 & 2 & 60 & 45 & 220 & 3 & 1 & 20 & 45 \\
\hline 141 & 2 & 0.5 & 5 & 60 & 181 & 2 & 2 & 80 & 45 & 221 & 3 & 1 & 40 & 45 \\
\hline 142 & 2 & 0.5 & 10 & 60 & 182 & 2 & 2 & 100 & 45 & 222 & 3 & 1 & 60 & 45 \\
\hline 143 & 2 & 0.5 & 20 & 60 & 183 & 2 & 2 & 5 & 60 & 223 & 3 & 1 & 80 & 45 \\
\hline 144 & 2 & 0.5 & 40 & 60 & 184 & 2 & 2 & 10 & 60 & 224 & 3 & 1 & 100 & 45 \\
\hline 145 & 2 & 0.5 & 60 & 60 & 185 & 2 & 2 & 20 & 60 & 225 & 3 & 1 & 5 & 60 \\
\hline 146 & 2 & 0.5 & 80 & 60 & 186 & 2 & 2 & 40 & 60 & 226 & 3 & 1 & 10 & 60 \\
\hline 147 & 2 & 0.5 & 100 & 60 & 187 & 2 & 2 & 60 & 60 & 227 & 3 & 1 & 20 & 60 \\
\hline 148 & 2 & 1 & 5 & 30 & 188 & 2 & 2 & 80 & 60 & 228 & 3 & 1 & 40 & 60 \\
\hline 149 & 2 & 1 & 10 & 30 & 189 & 2 & 2 & 100 & 60 & 229 & 3 & 1 & 60 & 60 \\
\hline 150 & 2 & 1 & 20 & 30 & 190 & 3 & 0.5 & 5 & 30 & 230 & 3 & 1 & 80 & 60 \\
\hline 151 & 2 & 1 & 40 & 30 & 191 & 3 & 0.5 & 10 & 30 & 231 & 3 & 1 & 100 & 60 \\
\hline 152 & 2 & 1 & 60 & 30 & 192 & 3 & 0.5 & 20 & 30 & 232 & 3 & 2 & 5 & 30 \\
\hline 153 & 2 & 1 & 80 & 30 & 193 & 3 & 0.5 & 40 & 30 & 233 & 3 & 2 & 10 & 30 \\
\hline 154 & 2 & 1 & 100 & 30 & 194 & 3 & 0.5 & 60 & 30 & 234 & 3 & 2 & 20 & 30 \\
\hline 155 & 2 & 1 & 5 & 45 & 195 & 3 & 0.5 & 80 & 30 & 235 & 3 & 2 & 40 & 30 \\
\hline 156 & 2 & 1 & 10 & 45 & 196 & 3 & 0.5 & 100 & 30 & 236 & 3 & 2 & 60 & 30 \\
\hline 157 & 2 & 1 & 20 & 45 & 197 & 3 & 0.5 & 5 & 45 & 237 & 3 & 2 & 80 & 30 \\
\hline 158 & 2 & 1 & 40 & 45 & 198 & 3 & 0.5 & 10 & 45 & 238 & 3 & 2 & 100 & 30 \\
\hline 159 & 2 & 1 & 60 & 45 & 199 & 3 & 0.5 & 20 & 45 & 239 & 3 & 2 & 5 & 45 \\
\hline 160 & 2 & 1 & 80 & 45 & 200 & 3 & 0.5 & 40 & 45 & 240 & 3 & 2 & 10 & 45 \\
\hline
\end{tabular}


Table D-2. Full Factorial experimental design for face wrinkling response of sandwich columns compressed perpendicular-to-corrugations under $\mathrm{V}=0.1 \mathrm{~m} / \mathrm{s}$ and $\mathrm{V}=1 \mathrm{~m} / \mathrm{s}$ (continued).

\begin{tabular}{ccccc|ccccc|cccccc}
\hline $\begin{array}{c}\text { Exp. } \\
\text { No. }\end{array}$ & $\mathrm{h}$ & $\mathrm{t}$ & $\mathrm{l}$ & $\mathrm{w}$ & $\begin{array}{c}\text { Exp. } \\
\text { No. }\end{array}$ & & & $\mathrm{h}$ & $\mathrm{t}$ & $\mathrm{l}$ & $\mathrm{w}$ & $\begin{array}{c}\text { Exp. } \\
\text { No. }\end{array}$ & $\mathrm{h}$ & $\mathrm{t}$ & $\mathrm{l}$ \\
\hline \hline 241 & 3 & 2 & 20 & 45 & 245 & 3 & 2 & 100 & 45 & 249 & 3 & 2 & 40 & 60 \\
242 & 3 & 2 & 40 & 45 & 246 & 3 & 2 & 5 & 60 & 250 & 3 & 2 & 60 & 60 \\
243 & 3 & 2 & 60 & 45 & 247 & 3 & 2 & 10 & 60 & 251 & 3 & 2 & 80 & 60 \\
244 & 3 & 2 & 80 & 45 & 248 & 3 & 2 & 20 & 60 & 252 & 3 & 2 & 100 & 60 \\
\hline
\end{tabular}


Table D-3. Full factorial experimental design for global buckling response of sandwich columns compressed parallel-to-corrugations under $\mathrm{V}=0.1 \mathrm{~m} / \mathrm{s}$ and $\mathrm{V}=1 \mathrm{~m} / \mathrm{s}$.

\begin{tabular}{|c|c|c|c|c|c|c|c|c|c|c|c|c|c|c|}
\hline $\begin{array}{l}\text { Exp. } \\
\text { No. }\end{array}$ & $\mathrm{h}$ & $\mathrm{t}$ & 1 & $\mathrm{w}$ & $\begin{array}{l}\text { Exp. } \\
\text { No. }\end{array}$ & $\mathrm{h}$ & $\mathrm{t}$ & 1 & $\mathrm{w}$ & $\begin{array}{l}\text { Exp. } \\
\text { No. }\end{array}$ & $\mathrm{h}$ & $\mathrm{t}$ & 1 & $\mathrm{w}$ \\
\hline 1 & 0.5 & 0.5 & 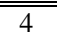 & 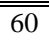 & 31 & 1.25 & 1.25 & 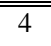 & 260 & 261 & 2 & 2 & 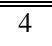 & 260 \\
\hline 2 & 0.5 & 0.5 & 8 & 60 & 32 & 1.25 & 1.25 & 8 & 60 & 62 & 2 & 2 & 8 & 60 \\
\hline 3 & 0.5 & 0.5 & 12 & 60 & 33 & 1.25 & 1.25 & 12 & 60 & 63 & 2 & 2 & 12 & 60 \\
\hline 4 & 0.5 & 0.5 & 16 & 60 & 34 & 1.25 & 1.25 & 16 & 60 & 64 & 2 & 2 & 16 & 60 \\
\hline 5 & 0.5 & 0.5 & 20 & 60 & 35 & 1.25 & 1.25 & 20 & 60 & 65 & 2 & 2 & 20 & 60 \\
\hline 6 & 0.5 & 0.5 & 25 & 60 & 36 & 1.25 & 1.25 & 25 & 60 & 66 & 2 & 2 & 25 & 60 \\
\hline 7 & 0.5 & 0.5 & 30 & 60 & 37 & 1.25 & 1.25 & 30 & 60 & 67 & 2 & 2 & 30 & 60 \\
\hline 8 & 0.5 & 0.5 & 35 & 60 & 38 & 1.25 & 1.25 & 35 & 60 & 68 & 2 & 2 & 35 & 60 \\
\hline 9 & 0.5 & 0.5 & 40 & 60 & 39 & 1.25 & 1.25 & 40 & 60 & 69 & 2 & 2 & 40 & 60 \\
\hline 10 & 0.75 & 0.75 & 50 & 60 & 40 & 1.25 & 1.25 & 50 & 60 & 70 & 2 & 2 & 50 & 60 \\
\hline 11 & 0.75 & 0.75 & 4 & 60 & 41 & 1.5 & 1.5 & 4 & 60 & & & & & \\
\hline 12 & 0.75 & 0.75 & 8 & 60 & 42 & 1.5 & 1.5 & 8 & 60 & & & & & \\
\hline 13 & 0.75 & 0.75 & 12 & 60 & 43 & 1.5 & 1.5 & 12 & 60 & & & & & \\
\hline 14 & 0.75 & 0.75 & 16 & 60 & 44 & 1.5 & 1.5 & 16 & 60 & & & & & \\
\hline 15 & 0.75 & 0.75 & 20 & 60 & 45 & 1.5 & 1.5 & 20 & 60 & & & & & \\
\hline 16 & 0.75 & 0.75 & 25 & 60 & 46 & 1.5 & 1.5 & 25 & 60 & & & & & \\
\hline 17 & 0.75 & 0.75 & 30 & 60 & 47 & 1.5 & 1.5 & 30 & 60 & & & & & \\
\hline 18 & 0.75 & 0.75 & 35 & 60 & 48 & 1.5 & 1.5 & 35 & 60 & & & & & \\
\hline 19 & 0.75 & 0.75 & 40 & 60 & 49 & 1.5 & 1.5 & 40 & 60 & & & & & \\
\hline 20 & 0.75 & 0.75 & 50 & 60 & 50 & 1.5 & 1.5 & 50 & 60 & & & & & \\
\hline 21 & 1 & 1 & 4 & 60 & 51 & 1.75 & 1.75 & 4 & 60 & & & & & \\
\hline 22 & 1 & 1 & 8 & 60 & 52 & 1.75 & 1.75 & 8 & 60 & & & & & \\
\hline 23 & 1 & 1 & 12 & 60 & 53 & 1.75 & 1.75 & 12 & 60 & & & & & \\
\hline 24 & 1 & 1 & 16 & 60 & 54 & 1.75 & 1.75 & 16 & 60 & & & & & \\
\hline 25 & 1 & 1 & 20 & 60 & 55 & 1.75 & 1.75 & 20 & 60 & & & & & \\
\hline 26 & 1 & 1 & 25 & 60 & 56 & 1.75 & 1.75 & 25 & 60 & & & & & \\
\hline 27 & 1 & 1 & 30 & 60 & 57 & 1.75 & 1.75 & 30 & 60 & & & & & \\
\hline 28 & 1 & 1 & 35 & 60 & 58 & 1.75 & 1.75 & 35 & 60 & & & & & \\
\hline 29 & 1 & 1 & 40 & 60 & 59 & 1.75 & 1.75 & 40 & 60 & & & & & \\
\hline 30 & 1 & 1 & 50 & 60 & 60 & 1.75 & 1.75 & 50 & 60 & & & & & \\
\hline
\end{tabular}


Table D-4. Full factorial experimental design for local plate buckling response of sandwich columns compressed parallel-to-corrugations under $\mathrm{V}=0.1 \mathrm{~m} / \mathrm{s}$ and $\mathrm{V}=1 \mathrm{~m} / \mathrm{s}$.

\begin{tabular}{ccccc|ccccc|ccccc}
\hline $\begin{array}{c}\text { Exp. } \\
\text { No. }\end{array}$ & $\mathrm{h}$ & $\mathrm{t}$ & $\mathrm{l}$ & $\mathrm{w}$ & $\begin{array}{c}\text { Exp. } \\
\mathrm{No}\end{array}$ & & $\mathrm{h}$ & $\mathrm{t}$ & $\mathrm{l}$ & $\mathrm{w}$ & $\begin{array}{c}\text { Exp. } \\
\text { No }\end{array}$ & $\mathrm{h}$ & $\mathrm{t}$ & $\mathrm{l}$ \\
\hline \hline 1 & 0.5 & 0.5 & 20 & 60 & 8 & 0.5 & 0.5 & 30 & 60 & 15 & 0.5 & 0.5 & 40 & 60 \\
2 & 0.75 & 0.75 & 20 & 60 & 9 & 0.75 & 0.75 & 30 & 60 & 16 & 0.75 & 0.75 & 40 & 60 \\
3 & 1 & 1 & 20 & 60 & 10 & 1 & 1 & 30 & 60 & 17 & 1 & 1 & 40 & 60 \\
4 & 1.25 & 1.25 & 20 & 60 & 11 & 1.25 & 1.25 & 30 & 60 & 18 & 1.25 & 1.25 & 40 & 60 \\
5 & 1.5 & 1.5 & 20 & 60 & 12 & 1.5 & 1.5 & 30 & 60 & 19 & 1.5 & 1.5 & 40 & 60 \\
6 & 1.75 & 1.75 & 20 & 60 & 13 & 1.75 & 1.75 & 30 & 60 & 20 & 1.75 & 1.75 & 40 & 60 \\
7 & 2 & 2 & 20 & 60 & 14 & 2 & 2 & 30 & 60 & 21 & 2 & 2 & 40 & 60 \\
\hline
\end{tabular}




\section{APPENDIX E THE EQUIVALENT CORE STIFFNESS OF THE CORE STRUT CLOSEST TO THE FRONT END}

The first core ligament can be modelled as below.

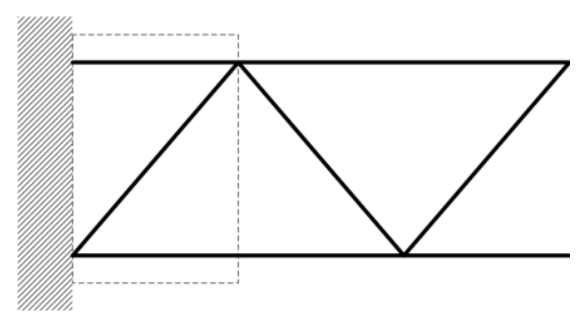

(a)

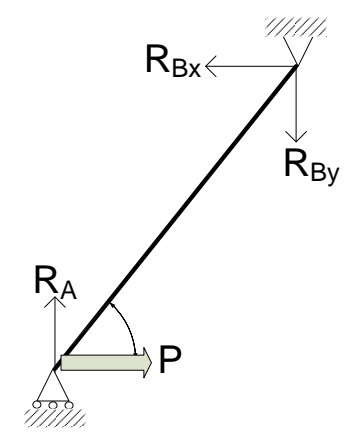

(b)

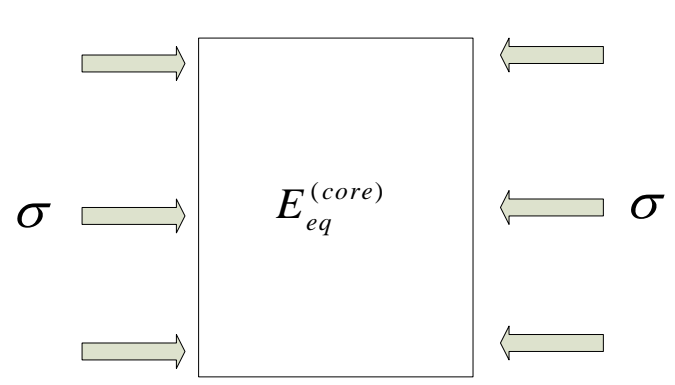

(c)

Figure E1: The equivalent in-plane core property for the high velocity.

From the force and moment equilibrium,

$$
\begin{gathered}
\sum F_{x}=0 ; \quad \mathrm{R}_{\mathrm{Bx}}=P \\
\sum M_{z}=0 ; \quad P l \sin \omega=R_{A y} \cdot l \cos \omega \\
\therefore R_{A y}=P \cdot \tan \omega \\
\therefore \delta_{\text {core_strut }}=\left(\frac{P / \cos \omega}{t E /\left(1-v^{2}\right)}\right) \ell
\end{gathered}
$$

From the equivalent strain energy relation,

$$
\begin{aligned}
\frac{1}{2}\left(\frac{P}{\cos \omega}\right) \delta_{\text {core_lig }} & =\frac{1}{2}\left(\frac{\sigma^{2}}{E_{\text {core }}^{*}}\right) \cdot\left[l^{2} \cos \omega \cdot \sin \omega\right] \\
& =\frac{1}{2}\left(\frac{1}{E_{\text {core }}^{*}}\right)\left(\frac{P}{l \sin \omega}\right)^{2} \cdot\left[l^{2} \cos \omega \cdot \sin \omega\right]
\end{aligned}
$$


Substituting Eqn. (E2) into (E3), the equivalent core stiffness is:

$$
\therefore E_{\text {core }}^{*}=\frac{(\cos \omega)^{3}}{\sin \omega} \frac{t E}{l\left(1-v^{2}\right)}
$$

The final equation (E4) is consistent with Balawi [96]'s Equivalent $C_{11}$ for triangle honeycomb. For example, the equivalent corrugated core stiffness of Al6061-T6 corrugated core having relative core density $\bar{\rho}=0.25$ is $E_{\text {core }}^{*}=1757.04 \mathrm{MPa}$. 
APPENDIX F : ANALYTIC EXPRESSION FOR PEAK REACTION FORCES IN A RATE-INDEPENDENT ELASTIC-PLASTIC MATERIAL WITH TWO HARDENING MODULI

According to the rate-independent theory of wave propagation [70-73] in an onedimensional rod,

$$
V=\int_{0}^{\varepsilon} c(\varepsilon) \cdot d \varepsilon
$$

Rewriting Eqn. (F1) for a rate-independent elastic-plastic material with two plastic hardening moduli with the assumption of axial stress-strain behavior as shown in Fig. 7.14,

$$
\begin{aligned}
& V=c_{e l}^{\prime(0)} \varepsilon_{Y}^{\prime(0)}+c_{p l}^{\prime(1)}\left(\varepsilon_{Y}^{\prime(1)}-\varepsilon_{Y}^{\prime(0)}\right)+c_{p l}^{\prime(2)}\left(\varepsilon-\varepsilon_{Y}^{\prime(1)}\right) \\
& \varepsilon_{Y}^{\prime(0)}=\frac{P_{Y}^{\prime(0)}}{(A E)_{e f f}^{\prime(0)}} \\
& \varepsilon_{Y}^{\prime(1)}-\varepsilon_{Y}^{\prime(0)}=\frac{P_{Y}^{\prime(1)}-P_{Y}^{\prime(0)}}{(A E)^{\prime(1)}} \\
& \varepsilon-\varepsilon_{Y}^{\prime(1)}=\frac{P^{(\text {front })}-P_{Y}^{(1)}}{(A E)^{\prime(2)}}
\end{aligned}
$$

Substituting Eqns. (F3a),(F3b),(F3c) into Eqn. (F2),

$$
\frac{P^{(f r o n t)}}{P_{Y}^{\prime(0)}}=\frac{P_{Y}^{\prime(1)}}{P_{Y}^{\prime(0)}}-\sqrt{\frac{(A E)_{e f f}^{\prime(2)}}{(A E)_{e f f}^{\prime(1)}}}\left(\frac{P_{Y}^{\prime(1)}-P_{Y}^{\prime(0)}}{P_{Y}^{\prime(0)}}\right)+\sqrt{\frac{(A E)_{e f f}^{\prime(2)}}{(A E)_{e f f}^{\prime(0)}}}\left(\frac{V}{c_{e l}^{\prime(0)} \varepsilon_{Y}^{\prime(0)}}-1\right)
$$


Upper bound of back end reaction force can be obtained considering wave reflection of elastic stress wave and Eqn. (F1).

$$
\begin{gathered}
c_{e l}^{\prime(0)} \mathcal{E}_{Y}^{\prime(0)}=c_{p l}^{\prime(1)} \cdot\left(\varepsilon_{Y}^{\prime(1)}-\varepsilon_{Y}^{\prime(0)}\right)+c_{p l}^{\prime(2)} \cdot\left(\varepsilon-\varepsilon_{Y}^{\prime(1)}\right) \\
\frac{P_{\text {upper }}^{(\text {back })}}{P_{Y}^{\prime(0)}} \approx \frac{P_{Y}^{\prime(1)}}{P_{Y}^{\prime(0)}}+\sqrt{\frac{(A E)_{e f f}^{\prime(2)}}{(A E)_{e \text { eff }}^{\prime(0)}}}-\sqrt{\frac{(A E)_{e f f}^{\prime(2)}}{(A E)_{e \text { eff }}^{\prime(1)}}} \cdot \frac{P_{Y}^{\prime(1)}-P_{Y}^{\prime(0)}}{P_{Y}^{\prime(0)}} \\
\frac{P_{\text {lower }}^{(\text {back }}}{P_{Y}^{\prime(0)}} \approx 1
\end{gathered}
$$

